\title{
Ultrafast vibrational spectroscopy of the eumelanin pigment
}

by

Karen Elisabeth Thorn

\author{
Thesis \\ submitted to the Victoria University of Wellington \\ in fulfilment of the \\ requirements for the degree of \\ Doctor of Philosophy \\ in Physics.
}

Victoria University of Wellington

5 March 2021 
Page 0 


\section{Acknowledgements}

I gratefully acknowledge project research funding from the Marsden Grant (VUW1620) as well as research support from the MacDiarmid Institute for Advanced Materials and Nanotechnology and the Victoria University of Wellington.

I thank my family for their constant support throughout my PhD research - my partner Ronnie Tamming, my parents Alison and Neville, my sisters Jennifer and Barbara, and my three wonderful children, Arthur, Leonard and Cassidy. My two cats, Moonlight Thunderstruck and Boris, also must be mentioned having entertained me through many all-nighters spent writing this thesis!

I thank my research team and peers working in the Ultrafast Laser Spectroscopy group in the School of Chemical and Physical Sciences, particularly the guidance given by Dr. Kai Chen, Dr. Paul Hume, Dr. Michael Price, Shyamal Prasad and Dr. Nick Monahan. My laser-lab PhD peers Ronnie Tamming, Sreelakshmi Sandrabose, Aleksandra Ilina, Isabella Wagner and Clément Sester have made this research project not only intellectually stimulating but also just so much fun.

I am grateful for the research collaborations established throughout this project - in particular with the Raman Laboratory at the Victoria University of Wellington led by Professor Eric Le Ru, with much work in our spontaneous Raman studies of eumelanin being performed by Dr. Johan Grand (as well as constant loans of equipment!). Additionally, collaborations with the Chemistry Department at the University of Otago led by Professor Keith Gordon, with the assistance of Dr. Joshua Sutton, has been essential to our resonance Raman studies. I can't thank warmly enough the gracious hospitably I received at the Institute of Physical and Chemical Research at the Riken Laboratory in Tokyo, Japan, under the guidance of Chief Scientist and Director of the Molecular Spectroscopy Laboratory Professor Tahei Tahara. Additionally I warmly thank Dr. Hikaru Kuramochi and Dr. Pardeep Kumar for their many hours of research spent with me on 
this project.

Lastly, I would like to acknowledge the ongoing support and guidance of my primary supervisor, Professor Justin Hodgkiss, and secondary supervisor, Dr Baptiste Auguié, and am grateful to the Victoria University of Wellington for providing state of the art scientific research programmes, and an ongoing support in the promotion of women in science. 


\section{Abstract}

Solar ultraviolet (UV) radiation is a highly toxic carcinogen prevalent in our environment. Eumelanin pigment is a photo-stable biopolymer naturally produced in the skin's pigmentary system, providing the skin a unique photo-protection mechanism against exposure to UV radiation. The large macro-molecule rapidly dissipates $\sim 99 \%$ of incident UV photons as thermal energy on ultra-fast femtosecond $\left(f_{s}\right)$ - picosecond (ps) time scales, before damage can occur to the underlying cells. The fundamental nature of eumelanin's structure and its vibrational energy dissipation mechanism is not yet fully understood, with complexities in the molecule's highly disordered chemical structure, and the ultrafast time-scale on which the energy dissipation occurs, rendering its characterisation elusive.

Indeed, the absorption spectrum of eumelanin gives little away, rising monotonically in wavelength towards the UV - quite unusual in organic polymers. It is proposed that due to the highly disordered structure of the molecule, multiple chromophores of overlapping energies may be selectively excited with differing irradiation wavelengths. This theory is further supported by eumelanin's transient absorption signatures and its wavelength dependant photo-luminescence spectra, however the fundamental non-radiative relaxation pathways are not yet understood.

To bridge this knowledge gap, we present here the application of femtosecond stimulated Raman spectroscopy (FSRS) to eumelanin pigment. FSRS reveals ultra-fast vibrational dynamics on $f s-p s$ time scales, allowing excited state vibrational pathways to be mapped providing essential structural information of this intriguing molecule.

Following the introduction of eumelanin's known photo-physical and structural properties as presented in chapter 1, an introduction to FSRS is presented in chapter 2 . The build method and optical construction of the FSRS experiment are presented in chapter 4. including a novel bandwidth compression method used to generate a narrow-band 
Raman pump using frequency-domain nonlinear optics, presented in chapter 3 . Here, using a $1.5 \mathrm{kHz}, 800 \mathrm{~nm}$ Ti:Sapphire pulsed laser at a power of $3 \mathrm{~W}$, conversion efficiencies of up to $30 \%$ are achieved, generating intense second harmonic Raman pump pulses centred at $400 \mathrm{~nm}$ with $<20 \mathrm{~cm}^{-1}$ bandwidths. Additionally, the well-known spatial filtering technique is used to generate tuneable, narrow-band pulses centered at the fundamental $800 \mathrm{~nm}$ laser pulse.

The application of FSRS to the study of eumelanin's indole subunits, 5-6-dihydroxyindole (DHI) and its carboxylated form, 5-6-dihydroxyindole-2-carboxylic acid (DHICA) (and their oligomers) are presented in chapter 5. These studies provide direct evidence for excited state proton transfer (ESPT) in DHICA, and a reference for the interpretation of the complex eumelanin macro-molecule, as well as its dynamic vibrational signatures, which are discussed in chapter 6. Here, vibrational dynamics are resolved on ps time scales, with the evolution of spectral features giving insight into eumelanin's vibrational energy dissipation mechanisms, showing that eumelanin's signatures relax via localised excited states. Also compared are the spectra and kinetics of eumelanin films and dispersions, which reveal a dependence on the molecule's surrounding environment.

From these FSRS studies, excited state proton transfer (ESPT) - proposed as a relaxation pathway in eumelanin's subunit DHICA, is demonstrated. Direct vibrational relaxation measurement mapped using density functional theory (DFT) provides strong evidence of excited state de-activation of DHICA via this mechanism. Further, eumelanin's vibrational mode deactivation pathways are presented, providing evidence of specific mode excitations of the macro-molecule upon photo-excitation in both the UV $(267 \mathrm{~nm})$ and visible $(400 \mathrm{~nm})$ regions.

Resolving eumelanin's excited state vibrational modes and kinetics using FSRS provides dynamic structural information of eumelanin's thermal energy transfer system, including that of its indole subunits. Determining FSRS signatures as a function of excitation wavelength in both the visible and UV regions, reveals insights into this highly efficient UV absorbing material. 


\section{Contents}

\begin{tabular}{ll}
\hline List of figures & 1
\end{tabular}

List of tables $\quad 5$

\begin{tabular}{ll}
\hline List of abbreviations & 7
\end{tabular}

$\begin{array}{lll}1 \text { Introduction } & 11\end{array}$

1.1 Research motivation . . . . . . . . . . . . . . . . . . . . 11

1.2 Eumelanin pigment . . . . . . . . . . . . . . . . . . 12

1.2 .1 Micro-structure of eumelanin; DHI and DHICA . . . . . . . . 12

1.2.2 Macro-molecular structural models for eumelanin . . . . . . . . . 14

1.2 .3 Photo-physical properties . . . . . . . . . . . . . . . . . . 16

1.2 .4 Absorption and emission . . . . . . . . . . . . . . . . 16

$1.2 .5 \quad$ Energy dissipation upon photo-excitation . . . . . . . . . . . 18

1.2.5.1 Excited state proton transfer . . . . . . . . . . . . 18

1.2.5.2 Proton coupled electron transfer . . . . . . . . . . . . 19

1.3 Method selection . . . . . . . . . . . . . . . . . . . . 20

1.4 Thesis outline $\ldots \ldots \ldots \ldots \ldots \ldots \ldots$

$\begin{array}{|ll|}2 & \text { Introduction to femtosecond stimulated Raman spectroscopy }\end{array}$

2.1 Chapter abstract . . . . . . . . . . . . . . . . . . . 23

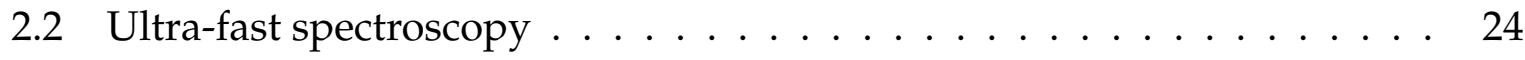

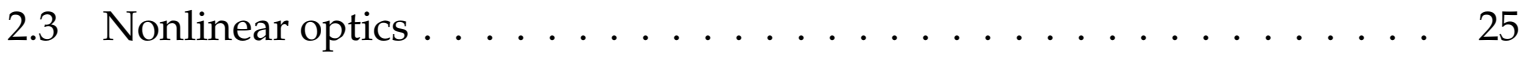

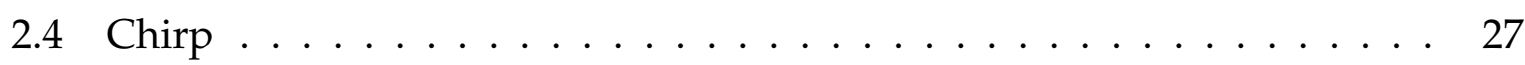

2.5 Femtosecond stimulated Raman spectroscopy $\ldots \ldots \ldots \ldots \ldots \ldots$

2.6 Chapter summary $\ldots \ldots \ldots \ldots \ldots \ldots \ldots \ldots$

3 A novel method to generate a narrowband Raman pump 35

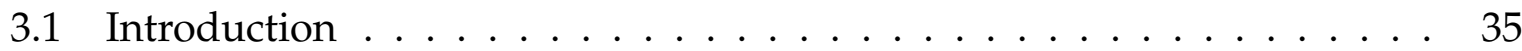


3.2 Raman pump generation using frequency-domain nonlinear optics . . . 35

3.3 Important aspects of the Raman pump . . . . . . . . . . . . . . . 36

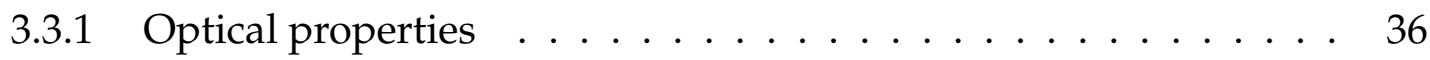

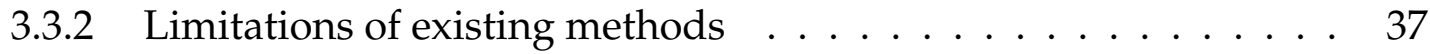

3.4 Method background $\ldots \ldots \ldots \ldots \ldots$. . . . . . . . . . . 37

3.4 .1 Sum frequency generation $\ldots \ldots \ldots . \ldots . \ldots . \ldots 37$

3.4 .2 Bandwidth compression model . . . . . . . . . . . . . . . . 39

3.4.2.1 Derivation for prism configuration . . . . . . . . . 39

3.4.2.2 Bandwidth compression simulation for the prism and grating configurations . . . . . . . . . . 4 43

3.5 Experimental configuration . . . . . . . . . . . . . . . 45

3.5 .1 Prism compressor. . . . . . . . . . . . . . . . . . . . 45

3.5 .2 Grating compressor - layout improvements . . . . . . . . . . . 46

3.6 Experimental results . . . . . . . . . . . . . . . . . . 47

3.6 .1 Spectral compression . . . . . . . . . . . . . . . . 47

3.6 .2 Tunability . . . . . . . . . . . . . . . . . . . . . . . 49

3.6 .3 Power conversion . . . . . . . . . . . . . . . . . 50

3.6 .4 Beam profile . . . . . . . . . . . . . . . . . 51

3.7 Application to stimulated Raman spectroscopy . . . . . . . . . . . . . . 51

3.7 .1 Experimental method . . . . . . . . . . . . . 52

3.7 .2 Experimental results . . . . . . . . . . . . . . . 53

3.8 Raman pump generation using spatial filtering . . . . . . . . . . . 53

3.8 .1 Generating an IR Raman pump . . . . . . . . . . . . . . . . . . . 53

3.8.2 Generating an IR Raman pump using a spatial filter . . . . . . . . 54

3.9 Chapter summary . . . . . . . . . . . . . 56

$4 \quad$ Femtosecond stimulated Raman spectroscopy - system build 59

4.1 Experimental configuration . . . . . . . . . . . . . . . 59

4.2 Pulse generation . . . . . . . . . . . . . . . . . . . . . . 61

$4.2 .1 \quad$ Raman pump . . . . . . . . . . . . . . . 61

4.2 .2 Excitation pump . . . . . . . . . . . . . . 61

4.2 .3 The probe: white light generation . . . . . . . . . . . . 63

4.3 Beam improvements . . . . . . . . . . . . . . . . . . . . . . . 64

4.3 .1 Spot size . . . . . . . . . . . . . . . . . 64

4.3 .2 Beam profile . . . . . . . . . . . . . . . . . 65

4.4 Data collection . . . . . . . . . . . . . . . . . . . . . 66

$4.4 .1 \quad$ High resolution spectroscopy $\ldots \ldots \ldots$. . . . . . . . . . . . 66 
4.4 .2 Shot collection . . . . . . . . . . . . . . . . . . . . . . . . 69

4.4 .3 Chopper referencing . . . . . . . . . . . . . . . . . 70

4.5 Data processing . . . . . . . . . . . . . . . . . . . . 72

4.5 .1 Wavelength calibration. . . . . . . . . . . . . . . 72

4.5 .2 Baseline removal . . . . . . . . . . . . . . . . . 74

4.5 .3 Solvent normalisation . . . . . . . . . . . . . . . 75

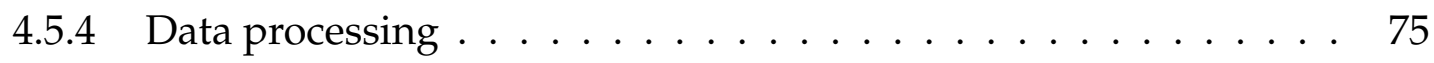

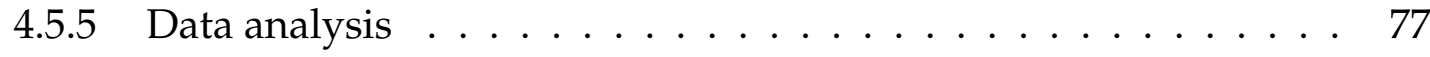

4.5.5.1 Singular value decomposition (SVD) analysis . . . . . 77

4.5.5.2 Density function theory (DFT) analysis . . . . . . . . 78

4.6 FSRS system test - napthalene $\ldots \ldots \ldots \ldots \ldots \ldots$

$4.6 .1 \quad$ Material background photophysics . . . . . . . . . . . 80

4.6 .2 Experimentation and results $\ldots \ldots \ldots \ldots \ldots$

4.6 .2 .1 Sample preparation . . . . . . . . . . . . . . 81

4.6.2.2 Analysis of ground state modes . . . . . . . . . . . . 81

4.6 .2 .3 Excited state FSRS analysis of napthalene . . . . . . . 82

4.7 Chapter summary $\ldots \ldots \ldots \ldots \ldots \ldots \ldots$

\begin{tabular}{|llr}
5 & Spectroscopic signatures of eumelanin subunits & 87
\end{tabular}

5.1 Introduction $\ldots \ldots \ldots \ldots \ldots \ldots \ldots \ldots \ldots$

5.2 Energy dissipation models $\ldots \ldots \ldots \ldots$. . . . . . . . . . . . 88

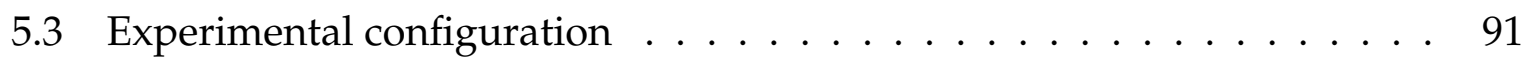

$5.3 .1 \quad$ FSRS experimental configurations $\ldots \ldots \ldots \ldots$. . . . . . 91

$5.3 .1 .1 \quad$ Sample preparations . . . . . . . . . . . . 92

5.3 .1 .2 Excitation pump configurations . . . . . . . . . . . 93

5.3 .1 .3 Spectrometer configurations . . . . . . . . . . . . . . 94

5.4 Experimental results $\ldots \ldots \ldots \ldots \ldots \ldots$

$5.4 .1 \quad$ Optical properties $\ldots \ldots \ldots \ldots \ldots \ldots$

$5.4 .1 .1 \quad$ Absorption . . . . . . . . . . . . . . . . . . . 94

5.4.1.2 Electronic properties: transient absorption . . . . . . . . 95

5.4 .2 Ground state Raman . . . . . . . . . . . . . . . . . . . . . 96

5.4.2.1 Ground state Raman spectra of DHI and DHICA . . . . 96

5.4.2.2 Mode assignment of the ground state using DFT analysis 98

5.4 .3 Time-resolved excited state Raman . . . . . . . . . . . . . . . 98

5.4.3.1 FSRS of DHI and DHICA in experimental configuration 198

5.4.3.2 $\quad$ Dynamic comparison for different solvents . . . . . . . 101

5.4 .3 .3 Evidence of excited state proton transfer (ESPT) . . . . . 104 
5.5 Chapter summary . . . . . . . . . . . . . . . . . . . . . . . . 109

6 Ultrafast vibrational spectroscopy of eumelanin pigment 111

6.1 Introduction . . . . . . . . . . . . . . . . . . . . . 111

6.2 Transient absorption of eumelanin films . . . . . . . . . . . . . . 112

6.2.1 Determining the optical Raman pump frequency for eumelanin . 114

6.3 Experimental configuration . . . . . . . . . . . . . . . . 116

$6.3 .1 \quad$ FSRS experimental configuration . . . . . . . . . . . . . . 116

6.3 .2 Sample preparation . . . . . . . . . . . . . . . . 116

6.3 .2 .1 Eumelanin film preparation . . . . . . . . . . . . 116

6.3 .2 .2 Eumelanin dispersion preparation . . . . . . . . . 117

6.3 .3 Pump details and spectrometer configuration . . . . . . . . . 118

$6.3 .3 .1 \quad$ Data processing . . . . . . . . . . . . . . . . . . . . 119

6.4 Experimental results . . . . . . . . . . . . . . . . . . . . . . . . . . 119

6.4 .1 Ground state Raman of eumelanin . . . . . . . . . . . . . . . . . 119

6.4.1.1 Ground state Raman band assignments. . . . . . . . . . 120

6.4.2 Comparisons between eumelanin and amorphous carbon materials 121

6.4 .2 .1 Resonance Raman analysis . . . . . . . . . . . . . . . 122

6.4 .3 Excited state vibrational mechanisms of eumelanin . . . . . . . 123

6.5 Discussion . . . . . . . . . . . . . . . . . . . . . . . 125

6.6 Heating effects in eumelanin films . . . . . . . . . . . . . . . . 131

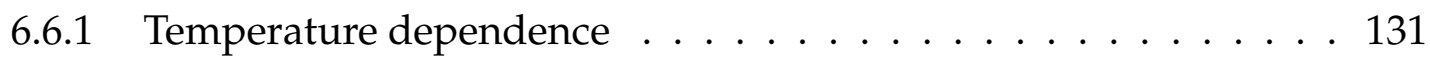

6.6.1.1 $\quad$ Estimating the temperature increase upon laser excitation 131

6.6.1.2 Temperature dependence of eumelanin's ground state Raman spectrum . . . . . . . . . . . . . . . . . . 134

6.6.2 A study of eumelanin using time-resolved impulsive stimulated Raman spectroscopy . . . . . . . . . . . . . . . . . . 136

6.7 Chapter summary . . . . . . . . . . . . . . . . . . . . . . . . 141

\begin{tabular}{lll}
\hline 7 & Research conclusions and outlook & 143
\end{tabular}

\begin{tabular}{|ll}
\hline A FSRS system build layout - technical drawings & 147
\end{tabular}

A.1 Complete FSRS optical system. . . . . . . . . . . . . . . . . . . . . 147

A.2 Raman pump generation - bandwidth compressor (grating configuration) 147

A.3 Excitation pump generation - second and third harmonic generation . . 147

A.4 Sample area and spectrometer - $400 \mathrm{~nm}$ Raman pump configuration. . . 147

Page viii 
B Matlab code used for FSRS data post-processing 153

B.1 Chopper referencing for the CMOS detector. . . . . . . . . . . . . 153

B.2 Chopper referencing for the PDA detector . . . . . . . . . . . . . . 156

B.3 Calibration of the CMOS detector using a standard . . . . . . . . . . 159

B.4 Calibration of the PDA (Stresing) detector using three notch filters. . . . 161

B.5 Baseline removal using asymmetric least squares approximation. . . . . 163

\begin{tabular}{|l|l|l|l|l}
\hline Matlab code used for FSRS data post-processing & 165
\end{tabular}

C.1 Derivative analysis of the FSRS surfaces . . . . . . . . . . . . . 165

\begin{tabular}{|ll}
\hline D Vibrational mode assignments of DHICA & 169
\end{tabular}

\begin{tabular}{|lll}
\hline E Pseudo-Voigt model for steady state mode assignments & 171
\end{tabular}

\begin{tabular}{lll}
\hline E.1 Pseudo-Voigt model parameters used for the ground state melanin film 171 & 171
\end{tabular}

E.2 pseudo-Voigt model code used for the ground state melanin film . . . . 172

\begin{tabular}{lll}
\hline F SVD analysis of eumelanin films and dispersions & 179
\end{tabular}

F.1 Comparison of the ground and excited state. . . . . . . . . . . . . 179

F.1.0.1 FSRS of eumelanin in dispersion. . . . . . . . . . . . . 182

\begin{tabular}{lll}
\hline F.1.0.2 Comparison of energy transfer for films and dispersions 185 &
\end{tabular}

G Eumelanin FSRS surfaces and differential analysis in ammonia dispersion 187 


\section{List of Figures}

1.1 The DHI and DHICA monomer building blocks of eumelanin pigment . 13

1.2 The preferential bonding sites of DHI and DHICA and example dimer structures . . . . . . . . . . . . . . . . . . 14

1.3 Two potential macro-molecular bonding structures of eumelanin . . . . 15

1.4 The $\pi$-stacked oligomer semiconductor model of the eumelanin macro-

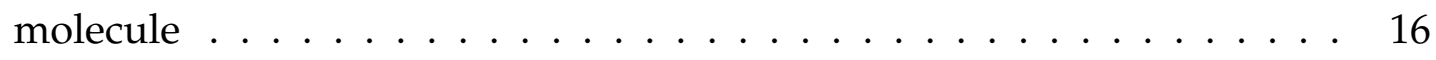

1.5 The optical absorption spectrum of eumelanin pigment . . . . . . . . . . 17

1.6 A model for excited state proton transfer in DHICA . . . . . . . . . . . 19

2.1 Temporal and spatial chirp of a laser pulse . . . . . . . . . . . . . 28

2.2 An energy level description of the (Stokes-shifted) stimulated Raman

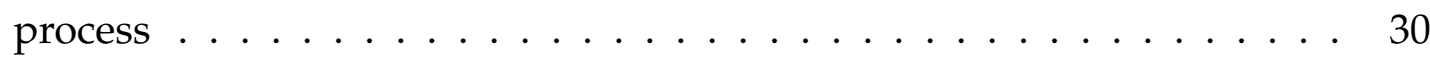

2.3 An energy level description of the femtosecond stimulated Raman process 31

3.1 Sum frequency generation of laser pulses with equal and opposite spatial chirp . . . . . . . . . . . . . . . . . . . 38

3.2 Geometric configurations for a focused Gaussian beam propagating through a nonlinear crystal . . . . . . . . . . . . . . . . . 40

3.3 Geometric configurations describing the spectral resolution of each colour at the BBO crystal, and the distortion factor caused by the crossing angle

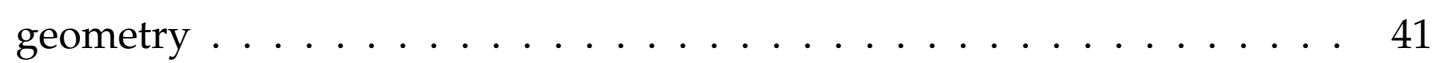

$3.4 \quad$ A graphical model for predicting the spectral bandwidth compression of the Raman pump bandwidth compressor for different system variables 44

3.5 Experimental configuration of the Raman pump bandwidth compressor (prism configuration) . . . . . . . . . . . . . 46

$3.6 \quad$ Experimental configuration of the Raman pump bandwidth compressor (grating configuration) . . . . . . . . . . . . . 47

3.7 Spectral compression and temporal broadening results from the Raman pump bandwidth compressor (prism and grating configurations) . . . . 48 
3.8 Fine and broad tuning results from the Raman pump bandwidth compressor, spanning the visible to the IR (prism configuration) . . . . . . . 49

3.9 Power conversion of the Raman pump bandwidth compressor (prism configuration) ......................... 50

3.10 Intensity profile of the Raman pump signal beam (prism configuration). 51

3.11 Experimental layout of the (ground state) femtosecond stimulated Raman spectroscopy system ....................... 52

3.12 Stimulated Raman spectra of methanol and acetone liquids . . . . . . . . 54

3.13 Experimental configuration of the $4 f$ spatial filter used to generate the Raman pump in the IR . . . . . . . . . . . . . . . . 55

3.14 Spectral bandwidth compression and power conversion of the spatial filter 56

4.1 The experimental configuration of the femtosecond stimulated Raman spectroscopy system . . . . . . . . . . . . . . 60

4.2 Experimental layout of the second and third harmonic generation sys-

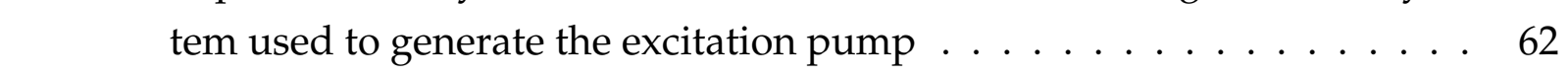

4.3 The spot size and fluence for each of the three laser beams at the sample

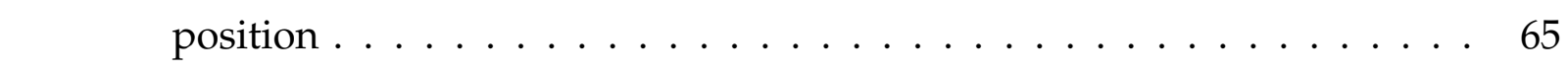

4.4 Corrections to the probe beam profile to reduce astigmatism at the sample 66

4.5 Schematic of the parameters used to determine optical resolution of the home-built spectrometers ................... 68

4.6 The mechanical chopping sequence used to generate a four shot sequence from the FSRS system for post-processing signal extraction . . . 70

4.7 Chopper referencing for the CMOS and PDA detectors . . . . . . . . 71

4.8 Calibration of the CMOS and PDA detectors using a solvent standard a nd notch filters . . . . . . . . . . . . . . . . 73

4.9 Baseline fitting and removal of the FSRS signals using an asymmetric least squares algorithm $\ldots \ldots \ldots \ldots \ldots \ldots \ldots$. . . . . . . . . . . . . . . .

4.10 Two step shot extraction and averaging process to extract the transient absorption, ground state stimulated Raman and excited state FSRS signals 76

4.11 UV-Vis absorption and excited state transient gain of napthalene for the FSRS system test . . . . . . . . . . . . . . . . . . . . . . . . . . . 80

4.12 Ground state Raman modes of napthalene . . . . . . . . . . . . . . . 81

4.13 DFT mode assignment of the ground state vibrational modes of naptha-

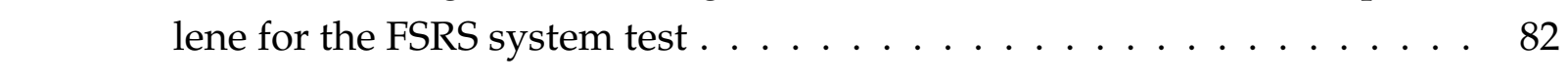

4.14 FSRS surface and time slices of napthalene in toluene for the FSRS sys-

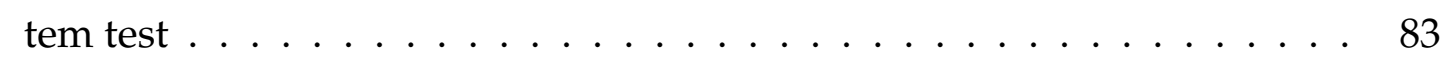


5.1 The structures of DHI and DHICA identifying phenol and carboxylic acid groups capable of proton transfer . . . . . . . . . . . . 89

5.2 An excited state proton transfer model for DHICA dissolved in water. . 90

5.3 UV-Vis absorption spectra of DHI and DHICA in water before and after

FSRS experiments . . . . . . . . . . . . . . . . 93

5.4 Transient absorption spectra and decay kinetics for DHI and DHICA in

\begin{tabular}{|c|}
\hline zater \\
\hline
\end{tabular}

5.5 Ground state Raman modes of DHI and DHICA plotted against expected peak locations from literature . . . . . . . . . . . . . . 97

5.6 Ground state vibrational mode assignments for DHI and DHICA from

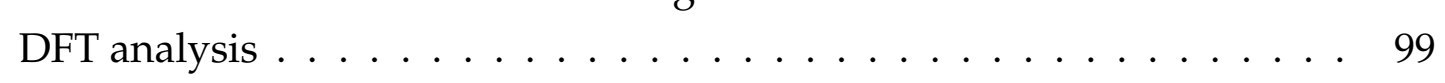

5.7 FSRS surfaces and SVD spectra and kinetics analysis for DHI and DHICA

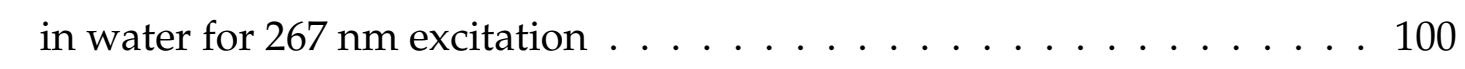

$5.8 \quad$ FSRS spectral traces for the DHI and DHICA monomers taken for five

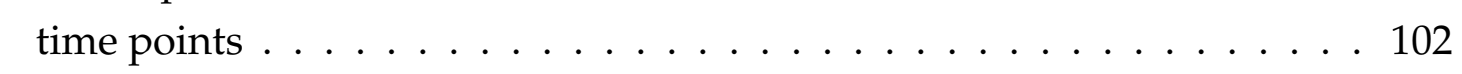

5.9 Kinetic traces of peak intensity dynamics for DHI and DHICA (in water) with DFT mode assignments . . . . . . . . . . . . . . . . 103

5.10 Spectral and kinetic traces of DHICA in water and in ethanol. . . . . . . 104

5.11 FSRS surfaces of DHICA in water and methanol . . . . . . . . . . . 105

5.12 DFT analysis of the excited state carboxylate anion and phenolate dianion DHICA molecules in the excited state proton transfer model . . . 106

5.13 Comparison of peak frequency shifts with DFT analysis of the excited state ESPT process . . . . . . . . . . . . . 107

5.14 Graphical representation of the DFT vibration modes around $1600 \mathrm{~cm}-1$ in the ESPT model . . . . . . . . . . . . . . . . . 108

6.1 Transient absorption spectra of eumelanin films for three excitation wave-

\begin{tabular}{|c|}
\hline lengths: $712 \mathrm{~nm}, 532 \mathrm{~nm}$ and $400 \mathrm{~nm}$; with decay kinetics for a $400 \mathrm{~nm}$ \\
\hline
\end{tabular}

6.2 Fluence dependent transient absorption spectra and kinetics of eumelanin 114

6.3 Comparison of eumelanin's ground state and excited state absorption spectra to determine the ideal Raman pump wavelength to achieve resonance enhancement of excited state vibrational modes . . . . . . . . . . 115

6.4 Measurement of the eumelanin film thickness . . . . . . . . . . . . . . . 117

6.5 UV-Vis absorption spectra for the eumelanin films and dispersions used in the FSRS experiments . . . . . . . . . . . . . . . . 118 
6.6 Eumelanin's ground state Raman spectra and pseudo-Voigt analysis of the vibrational bands . . . . . . . . . . . . . . . . . . . . 119

6.7 Ground state Raman spectra of amorphous carbon as an analogy to eumelanin's Raman spectra . . . . . . . . . . . . . . . . . . . . . 122

6.8 Ground state resonant Raman analysis of eumelanin films . . . . . . . 123

6.9 FSRS surface of melanin films for two excitation pump wavelengths . . 124

6.10 Comparison of eumelanin films at $267 \mathrm{~nm}$ and $400 \mathrm{~nm}$ excitations . . . . 125

6.11 Ground state Raman vs excited state Raman surfaces for eumelanin . . . 127

6.12 Comparison of DHICA in water and eumelanin film with DFT analysis of the excited state . . . . . . . . . . . . . . . 128

6.13 Comparison of the FSRS spectral slices of eumelanin films and DHICA in water for $267 \mathrm{~nm}$ excitation . . . . . . . . . . . . . . . . 130

6.14 A model for heat dissipation in eumelanin films and dispersions through - laser irradiation . . . . . . . . . . . . . . . . 133

6.15 Analysis of spontaneous Raman spectra of eumelanin films in the ground state for increasing temperatures . . . . . . . . . . . . . 136

6.16 Analysis of the ground state Raman band ratios for eumelanin films for increasing temperature . . . . . . . . . . . . . . 137

6.17 Optical configuration and key processing steps of the TR-ISRS system . 138

6.18 TS-ISRS experimental results for eumelanin films under $400 \mathrm{~nm}$ excitation 139

6.19 TS-ISRS experimental results for eumelanin dispersions under $400 \mathrm{~nm}$

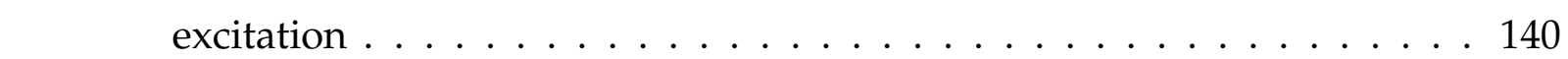

A.1 Technical drawing of the physical layout and optical components of the

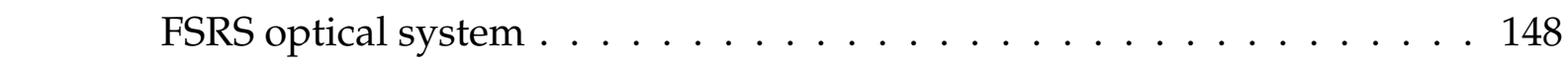

A.2 Technical drawing of the bandwidth compressor (grating configuration) 149

A.3 Technical drawing of the second and third harmonic generation system used to generate the excitation pump . . . . . . . . . . . . . . 150

A.4 Technical drawing of the sample area and spectrometer for the $400 \mathrm{~nm}$

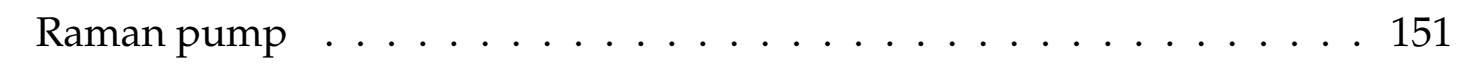

D.1 Ground state DFT vibrational mode assignments of the DHICA carboxylate anion and phenolate di-anion . . . . . . . . . . . 170

F.1 SVD analysis of eumelanin films for $267 \mathrm{~nm}$ and $400 \mathrm{~nm}$ excitations . . . 180

F.2 SVD analysis of eumelanin films for $267 \mathrm{~nm}$ and $400 \mathrm{~nm}$ excitations . . . 181

F.3 SVD spectral component analysis of eumelanin film for $267 \mathrm{~nm}$ excitation 182

F.4 SVD spectral component analysis of eumelanin film for $400 \mathrm{~nm}$ excitation 183 
F.5 Comparison of peak shifts and bandwidths for the SVD spectral components of eumelanin films and dispersions for $267 \mathrm{~nm}$ and $400 \mathrm{~nm}$ excitations . . . . . . . . . . . . . . . . . . . . 185

G.1 FSRS surfaces of melanin dispersions for two excitation pump wave-

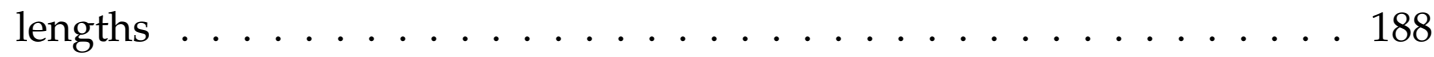

G.2 Comparison of eumelanin dispersions at $267 \mathrm{~nm}$ and $400 \mathrm{~nm}$ excitations 188 


\section{List of Tables}

3.1 Measured and predicted spectral and temporal bandwidths for the bandwidth compressor . . . . . . . . . . . . . . . 43

5.1 Experimental configurations for FSRS experiments on DHI and DHICA 91

5.2 Experimental Raman peak assignments for DHI and DHICA in the ground

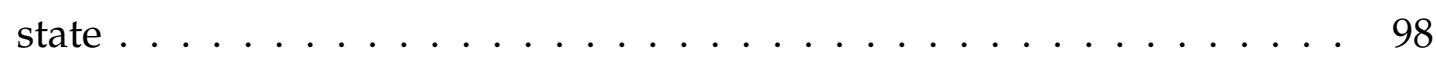

6.1 Central peak locations, and their corresponding mode assignments, are presented here for the 6 main peaks derived from a pseudo-Voigt function fitting analysis of eumelanin's broad-band, ground state peaks . . . 120

6.2 The calculated temperature rises for eumelanin films and dispersions . . 134

E.1 Pseudo-Voigt function parameters used to model the eumelanin pigment ground state Raman spectrum. . . . . . . . . . . . . . . . . . 171

F.1 Peak locations extracted from the Gaussian deconvolution of the 2 SVD components for eumelanin films at two excitation wavelengths $267 \mathrm{~nm}$ and $400 \mathrm{~nm} \ldots \ldots \ldots \ldots \ldots$. . . . . . . . . . . . . . 184

F.2 Peak locations extracted from the Gaussian deconvolution of the 2SVD components for eumelanin dispersions at two excitation wavelengths $267 \mathrm{~nm}$ and $400 \mathrm{~nm} \ldots \ldots \ldots \ldots$. . . . . . . . . . . 184 


\section{List of Abbreviations}

BBO beta barium borate.

CCF cross correlation function.

CT charge transfer.

$\mathrm{CW}$ continuous wave.

DHI 5-6-dihydroxyindole.

DHICA 5-6-dihydroxyindole-2-carboxylic acid.

ESPT Excited state proton transfer.

FSRS Femtosecond stimulated Raman spectroscopy.

FWHM full width at half maximum.

GSB ground state bleach.

GVD group velocity dispersion.

HOMO highest occupied molecular orbital.

IR infrared.

IRF instrument response function.

LUMO lowest unoccupied molecular orbital.

OR optical resolution.

PAH polycyclic aromatic hydrocarbon.

PCET Proton coupled electron transfer.

PES potential energy surface.

PIA photo-induced absorption. 
PL Photo-luminescence.

SFG sum frequency generation.

SHG second harmonic generation.

SNR signal-to-noise ratio.

TA transient absorption spectroscopy.

THG third harmonic generation.

TR-ISRS time-resolved impulsive stimulated Raman spectroscopy.

UV ultraviolet.

UV-Vis ultraviolet-visible optical absorption.

WLG white light generation.

YAG yttrium aluminium garnet. 


\section{Chapter 1}

\section{Introduction}

\subsection{Research motivation}

Solar ultraviolet (UV) radiation is prevalent in our environment as a highly toxic carcinogen with clear, detrimental effects in humans and surrounding ecosystems. Highly energetic UV photons can be absorbed by organic molecules and synthetic polymeric materials, and become photo-chemically active, leading to effects such as erythmia (sunburn), photo-aging (wrinkling) and the development of skin carcinomas ${ }^{[1] 4}$.

The skin's response to UV radiation is wavelength dependent across the spectrum, with some beneficial biological processes such as vitamin D and serotonin production, resulting from exposure to UVA $(320-400 \mathrm{~nm})$ radiation. It is well established that exposure to the more energetic UVB $(280-320 \mathrm{~nm})$ radiation leads to photo-damage of molecules, promoting the development of gene mutation and cell death. Cutaneous malignant melanoma, the most aggressive form of skin cancer, is the leading cause of cancer-related deaths in the world, with Australia and New Zealand having the highest mortality rate world-wide ${ }^{[5]}$. Cosmetic UV absorbers and blockers, while effective when correctly applied, have a limited lifetime and are heavily regulated due to ongoing debate regarding product efficacy across the full UVA - UVB spectrum, as well as potential toxicity to humans and the environment ${ }^{[6-9]}$.

Nature has provided humans with a natural photo-stable photoprotection mechanism against exposure to broadband UV radiation in the skin's pigmentary system. Eumelanin pigment, produced by melanocytes in the epidermis, is a complex brown-black biopolymer which rapidly dissipates $99.9 \%$ of incident UV photons as thermal energy before damage can occur to the underlying cells ${ }^{[10+13]}$. Production of eumelanin 
is triggered after UV exposure exceeds damage thresholds, causing the pseudopodial process (tanning), and individuals with eumelanin-rich skin have a naturally higher tolerance to UV photodamage, and therefore a lower cancer risk ${ }^{[14}$.

\section{Knowledge gap}

Despite its extremely efficient and effective function as a UV photo-protectant, the fundamental mechanism of eumelanin's energy dissipation process is not yet understood, with complexities in the molecule's chemical and physical structure, and the ultra-fast time-scale on which the energy dissipation occurs on the order of femtoseconds $(f s)$, or $10^{-15}$ seconds $(s)$, rendering characterisation of the transient excited state mechanics elusive to standard experimental methods.

\subsection{Eumelanin pigment}

\subsubsection{Micro-structure of eumelanin; DHI and DHICA}

Due to its highly disordered heterogeneous molecular structure and its virtual insolubility in solvents, chemical and structural characterisation of natural eumelanin pigment has provided science a challenge. It is widely accepted that eumelanin is composed of two indole building blocks: 5-6-dihydroxyindole (DHI) and its carboxylated form 5-6-dihydroxyindole-2-carboxylic acid (DHICA) and their varying redox forms $[10[12] 13] 15,19]$ as shown in figure 1.1 .

The two indole monomers can bond together in a variety of configurations, forming $\pi$-conjugated homo- and hetero- oligomers of different shapes, torsions and lengths on scales of $\sim 10-100 \mathrm{~nm}$. One secondary structural model for the eumelanin macromolecule is that of oligomers stacked together to form complex, aggregate layers of varying dimensions spaced $\sim 0.4 \mathrm{~nm}$ apart, likened to $\pi$ conjugated organic semiconductors 1011120-23]. This macro-molecular structure of extreme chemical disorder gives rise to the formation of discrete chromophores with overlapping HOMO-LUMO bandgaps - that is, the energy difference between the highest occupied molecular orbital (HOMO) or electronic ground state, and the lowest unoccupied molecular orbital (LUMO) or first electronic excited state - capable of absorbing photons across the UV and visible solar spectrum. Due to the complex and disordered nature of the macromolecule, most photo-physical and photo-chemical research into the functional aspects of the pigment to date have been undertaken on the soluble indole building blocks of 

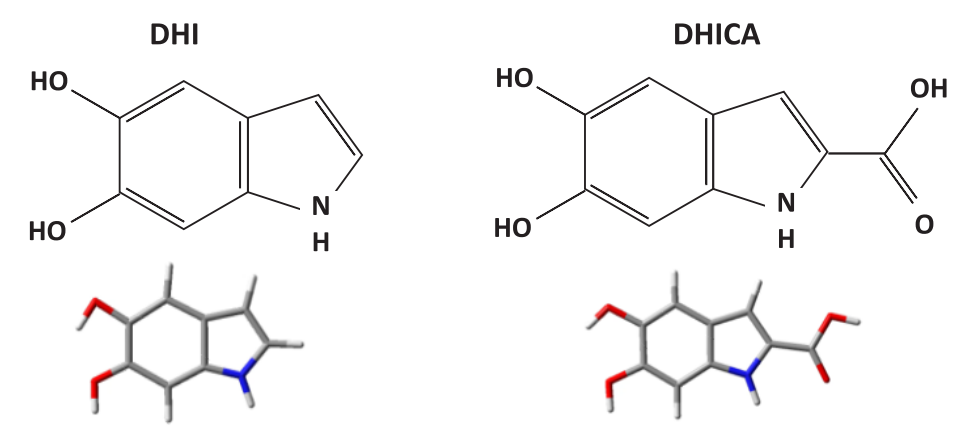

IQ

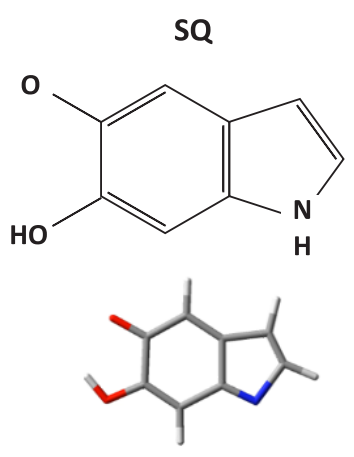

Figure 1.1: The main building blocks of eumelanin are shown. 5-6-dihydroxyindole (DHI) and its carboxylated form 5-6-dihydroxyindole-2-carboxylic acid (DHICA) are presented in the top panels, with DHI's quinone forms shown in the lower panels. These sub-units can from hetero-polymers and oligimors bonded in a variety of configurations.

eumelanin, or synthetic pigment replicas which are free from the residual protein coat bound to the natural material ${ }^{10]}$.

The primary structures of the DHI and DHICA sub-units shown in figure 1.1 can covalently bond to form monomers, with the favourable bonding positions of DHI being at the 2, 4 and 7 sites in DHI and the 4, 7 and 3 in DHICA as shown in figure 1.2 as the orange circles ${ }^{[1824]}$. Dimer, trimer and higher order homo- and hetero- polymers are formed through the preferential bonding locations. It is proposed that the DHICA monomer may be on the end of larger macro-molecular structural units due to its lack of available bonding sites in the $\mathrm{COOH}$ site ${ }^{[18[24]}$. 
DHI

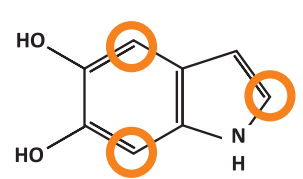

DHICA

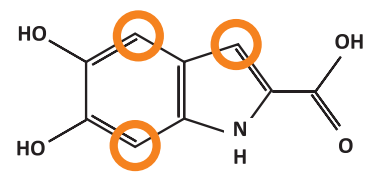

HO

preferred bonding positions

DHI 1

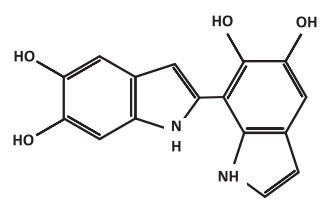

DHICA 1

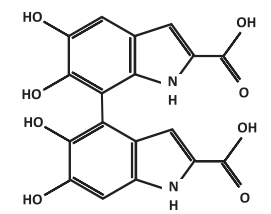

DHI 2
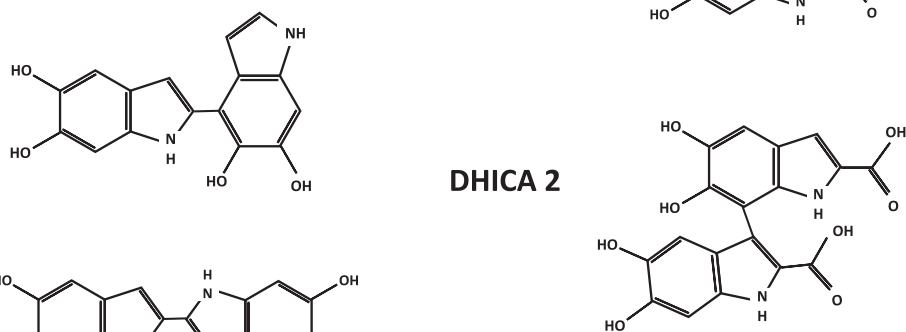

Figure 1.2: The preferential bonding sites of DHI and DHICA are indicated by orange circles. Examples of potential dimers formed in each subunit are shown below, labelled as DHI 1 - 3 and DHICA 1 - 2 .

\subsubsection{Macro-molecular structural models for eumelanin}

There are two proposed structural models as to how the DHI and DHICA building blocks assemble themselves in the eumelanin polymer. The first model is described as an extended, linear hetero-polymer formed through bonding of the DHI and DHICA monomers, while the second model is a $\pi$-conjugated system of stacked oligomer layers formed by DHI and DHICA, with approximately 4 - 6 monomers per oligomer chain, stacked in 4 - $5 \pi$ conjugated layers spaced $\sim 10 \AA$ apart 10/15/182425]. Spectroscopic studies, and extensive computational analysis, have not yet given conclusive favour to either model, with an added complexity that the macro-molecular structure of eumelanin may be a combination of both models.

In the bio-polymer (chemical disorder) model, the primary subunit structures associate together via Van der Walls forces or hydrogen bonding [2427]. This forms a larger structure as shown in the left hand panel of figure 1.3 . In the stacked oligomer 


\section{two potential bonding arrangements of eumelanin's sub-units}

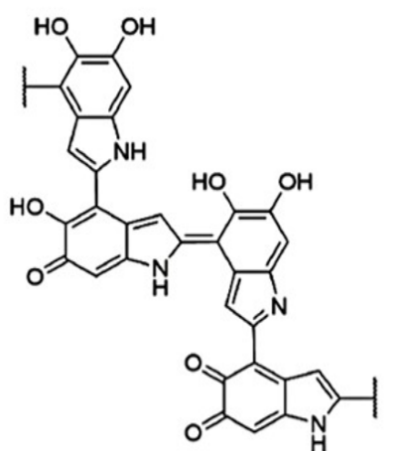

bio-polymer model

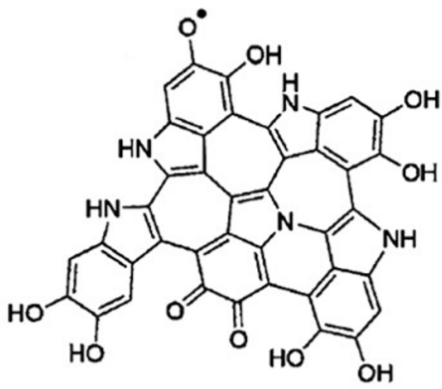

oligimor (stacking) model

Figure 1.3: Two potential bonding arrangements of the eumelanin sub-units to form the eumelanin macro-molecule are presented. The left hand side panel shows a covalently bonded polymeric chain, while the right hand side panel represents an oligomeric structure which then stacks together via $\pi$ orbital bonding. Both models suggest eumelanin to be a highly disordered, large polymer.

(semi-conductor) model conjugated bonds are formed through $\pi$ stacking of larger oligomers, as shown in figures 1.3 and 1.4 . Here, 3 stacked sheets of about $20 \AA$ wide and $7.6 \AA$ high are stacked together ${ }^{[2428]}$. Evidence for these larger hetero-polymeric structures have been supported using mass spectrometery experiments, as well as evidence of eumelanin's semi-conductor properties under doping [10[24]28].

Both models can account for the unique optical properties observed in eumelanin, and are still up for debate, especially in the observations of eumelanin's charge transfer properties. The assembly of the DHI and DHICA sub-units to form the eumelanin macro-molecule is further discussed in chapter 6, while in this chapter we focus on the individual characteristics of each subunit, particularly in terms of providing reference frameworks for eumelanin's dynamic vibrational relaxation signatures.

It should be noted that synthetic and natural eumelanin have different amounts of DHI versus DHICA (along with their quinone sub-groups) ${ }^{10 \mid 29}$. In this research, we are studying synthetic eumelanin where DHI and DHICA make up a ratio of $\sim 90: 10$ $\%$ of the disordered structure, compared to natural eumelanin with a ratio of $\sim 50: 50$ $\%$ of DHI to DHICA. 24 
stacked oligimor model of eumelanin

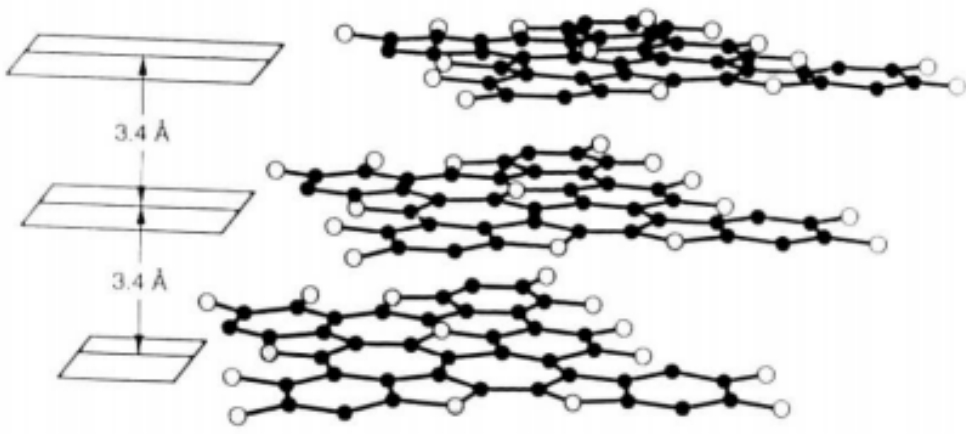

Figure 1.4: The stacked oligomer semiconductor model is presented. Here, layered oligomers a bound via $\pi$ conjugation, forming a large, macro-molecule capable of conducting charge. This figure is reproduced from the publication cited ${ }^{[26]}$ with permission by John Wiley \& Sons - Books provided by Copyright Clearance Center ("CCC").

What is agreed is that eumelanin is a highly disordered and chemically complex molecule, capable of non-radiative dissipation of photon energy on rapid $f s$ - $p s$ time scales. This mechanism is of particular interest in this research where, through the application of FSRS, vibrational dynamics of the photon-excited molecule are observed in both eumelanin films and dispersions.

\subsubsection{Photo-physical properties}

\subsubsection{Absorption and emission}

Eumelanin's absorption spectrum, shown in figure 1.5 (for experimental details please refer to section 6.3.2 of chapter 6) features a broadband and monotonic spectrum, rising exponentially (in wavelength) towards the UV 10,30+32]. This is ideal for absorbing energy radiated from the sun across a wide spectral range, and acting as a natural photoprotection layer against the sun's detrimental effects. It has been proposed that this featureless absorption spectrum, uncommon of organic materials, can be attributed 


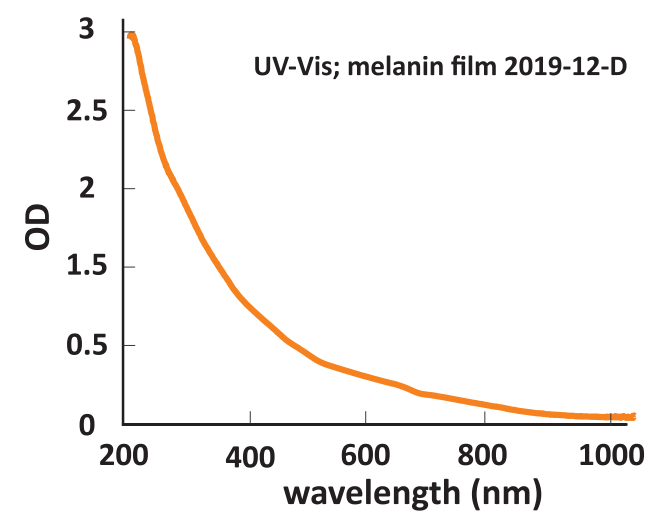

Figure 1.5: The ultraviolet-visible optical absorption (UV-Vis) spectrum of eumelanin pigment shows a monotonic rise towards the UV, unusual for organic polymers. This absorption spectrum is ideal for absorbing the sun's irradiation and gives eumelanin its unique brown pigmentation.

to contributions of many overlapping discrete chromophores, further supporting a macro-molecular structure of extreme chemical disorder ${ }^{[10]}$. Previous studies into the mechanisms behind eumelanin's broad absorption spectrum have tested the possibility of contributions from scattering, with contributions found to be less than $6 \%$ of the total optical attenuation between 210 and $325 \mathrm{~nm}^{\text {[32] }}$.

Photo-luminescence (PL) emission studies of eumelanin reveal an apparent violation of Kasha's rule which states that all radiated photons from an excited material must emit from the lowest excited state - the photon energies being therefore dependent on the material itself, rather than on excitation wavelength ${ }^{[33}$. In eumelanin, however, the PL spectrum shows distinct excitation wavelength dependence providing evidence, along with its broadband absorption spectrum, of distinct chromophores within the molecule being selectively activated upon light excitation, excluding charge transfer between chromophores ${ }^{[2834]}$.

Eumelanin's ground-state fluorescence emission is excitation wavelength dependent with extremely weak quantum yields of $<0.001 \%$ [3738]. This weak fluorescent signature provides evidence of rapid non-radiative energy dissipation pathways, however the vibrational dynamics of eumelanin's photo-physical dissipation mechanism have yet to be elucidated. 
Transient absorption spectroscopy ${ }^{[3940]}$ provides similar evidence of overlapping chromophores, in that the absorption energy of eumelanin mirrors that of the excitation wavelength, with a rapid $<p s$ decay. Conversely, eumelanin's photo-induced absorption (PIA) band peaks consistently at around $\sim 1.3 \mathrm{eV}$, shown in figure 6.3 of chapter 6, regardless of excitation wavelength. ${ }^{[14}$-44] Another interesting feature within the transient absorption spectroscopy (TA) dynamics is a lack of fluence dependence on eumelanin's electronic excited state decay, indicating a vast capability of the molecule to rapidly disperse photon energy even at extreme fluence levels. Further details of eumelanin's TA signatures are discussed in section 6.2 of chapter 6.

\subsubsection{Energy dissipation upon photo-excitation}

\subsubsection{Excited state proton transfer}

Excited state proton transfer (ESPT) is an energy dissipation mechanism whereby hydrogen atoms are lost from a molecule to a solvent bath on rapid $f_{s}-p s$ timescales. It was recently demonstrated by Sundström and coworkers that the carboxylated indole DHICA (DHICA-) dissolved in neutral $\mathrm{pH}$ undergoes ESPT after photoexcitation ${ }^{45-50]}$. A schematic of this process is shown in figure 1.6. Time-resolved ultrafast fluorescence spectroscopy is used to measure and compare the excited state decay of the Stokes shifted emission peaks in an aqueous buffer, with that of DHICA dissolved in methanol. It was found that in water, the excited state decay rate constant increased to $\sim 1.6 \mathrm{~ns}$ from $\sim 3.5 \mathrm{~ns}$ when compared with a methanol solution ${ }^{[45]}$. It is proposed that the differing decay mechanisms available to the DHICA- molecule is the ability of the $\mathrm{OH}$ phenol groups to deprotonate in water, a relaxation pathway not available in methanol.

Conducting similar time-resolved photo-luminescence studies as those performed on DHICA, it was noted a similar solvent dependence on DHI's excited state lifetimes, with $103 \mathrm{ps}$ measured in water, and $2.2 \mathrm{~ns}$ in methanol. It is proposed then, that a similar ESPT mechanism could be occurring in the DHI molecule as in DHICA ${ }^{51}$. Experimental referencing of this proposition is challenging, as oxidised DHI molecules rapidly polymerise in air and upon light excitation. The ESPT mechanism proposed for DHICA, and experimental results supporting this proposition, are further discussed in chapter 5 . 


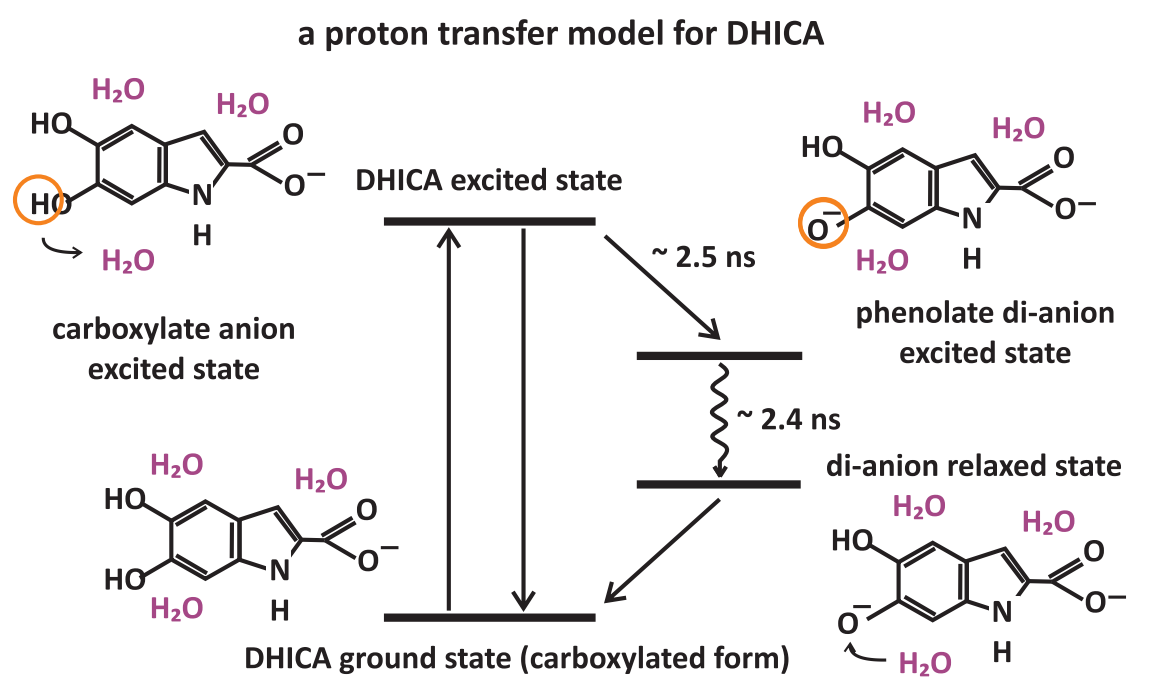

Figure 1.6: In the proton transfer model for the DHICA monomer, the relaxed sate forms an anion caused by the loss of the $\mathrm{H}+$ in the carboxylic acid to the solvent upon dispersion in water. Upon excitation, the phenol groups can lose protons to the solvent causing a rapid relaxation to a di-anion excited state. Further relaxation via fluorescence on a $n s$ time scale allows for scavenging protons back from the solvent to return to its ground state.

\subsubsection{Proton coupled electron transfer}

Proton coupled electron transfer (PCET) is a chemical reaction involving the sequential transfer of protons and electrons ${ }^{[52]}$. Sundström and coworkers have proposed that PCET could be an energy dissipation mechanism of DHI, in addition to ESPT, where upon UV excitation, a solvated electron is formed in the water solvent followed by the transfer of protons to the solvent from both phenol $\mathrm{OH}$ groups in the excited state $\mathrm{e}^{45}$. Internal conversion by way of return of the electron to the de-pronotated molecule sees the relaxation of the molecule back into the ground state.

Much of the proposition relies on computational analysis, as the optical study of DHI is inherently challenging due to its rapid polymerisation in air and upon light exposure, however spectroscopic measurements reveal a similar solvent dependence to that of DHICA, with energy dissipation times on the order of $100 \mathrm{ps}$ in an aqueous environment, 20 times faster than in methanol ${ }^{45[51]}$. 


\subsection{Method selection}

\section{Observing molecular vibrations using time-resolved Raman spectroscopy}

Arguably the most robust way to probe the true nature of DHI and DHICA's excited state deactivation mechanisms, and subsequently gain insight into the dissipation pathways of eumelanin pigment itself, is using a time-resolved ultrafast vibrational spectroscopy technique capable of directly resolving vibrational modes and kinetics on rapid $f s-p s$ timescales.

To directly probe eumelanin's excited state photophysical mechanism, an ultrafast spectroscopic method capable of resolving Raman spectra across its vibrational manifold on $f_{s}$ time-scales is required. Time-resolved ultrafast spectroscopy involves the use of pulsed lasers to interrogate the dynamics of chemical and photophysical reactions that occur on ultrashort time scales ${ }^{[53]}$. Specifically, time-resolved ultrafast Raman spectroscopy is used to probe vibrational modes that are coupled to a photochemically induced reaction, such as the absorption of a UV photon, and resolve structural dynamics throughout the molecule's vibrational dephasing time.

Femtosecond stimulated Raman spectroscopy is a spectroscopic tool capable of resolving excited state vibrational dynamics on ultrafast $f_{s}$ timescales ${ }^{[54}$ [58]. Applying this well established Femtosecond stimulated Raman spectroscopy (FSRS) method to eumelanin pigment can reveal the structural evolution of the non-radiative UV energy dissipation mechanism as a function of excitation wavelength, including the resolution of proton transfer.

\subsection{Thesis outline}

This thesis is set out as follows:

- Chapter 2 gives an introduction into ultra-fast spectroscopy including its general concept and a summary of the generation of ultra-short laser pulses, and the technique of transient absorption spectroscopy which is used as a spectroscopic tool in this research.

- Chapter 3 focuses on frequency domain second harmonic generation (SHG) used in the novel bandwidth compression technique for Raman pump in the visible $(400 \mathrm{~nm})$ and the spatial filtering technique used to generate an intense Ra- 
man pump in the IR $(800 \mathrm{~nm})$ used in the FSRS experiments conducted on DHI, DHICA and eumelanin in the following chapters.

- Chapter 4 provides full details of the FSRS system configuration, including the optical build, data collection, post processing software and data analysis methodologies.

- Chapter 5 gives details of experiments performed on DHI and DHICA, which shows direct evidence for excited state proton transfer in DHICA under neutral pH conditions.

- Chapter 6 extends this study to the eumelanin macro-molecule itself, with exciting evidence of ESPT in the large eumelanin structure, as referenced by FSRS experiments performed on DHICA.

- Finally, chapter 7 summarises this research project, showing that by using FSRS as a tool to reveal dynamic vibrational signatures in eumelanin and its subunits, we are one step closer to revealing the non-radiative dissipation mechanisms of eumelanin pigment. 
Page 22 


\section{Chapter 2}

\section{Introduction to femtosecond stimulated Raman spectroscopy}

\subsection{Chapter abstract}

In this chapter we introduce the ultra-fast vibrational spectroscopic method of femtosecond stimulated Raman spectroscopy (FSRS). We present the background of this method and how its application to eumelanin and its subunit monomers and oligomers may allow us to gain insights into eumelanin's non-radiative energy dissipation mechanisms.

This chapter is set out as follows:

- First, an introduction into ultra-fast spectroscopy is presented including its general concept, a brief summary of the generation of ultra-short laser pulses and an introduction into the technique of transient absorption spectroscopy which is used as a spectroscopic tool in this research.

- Secondly, an introduction into the phenomenon of nonlinear optics is presented, including a general theoretical overview, with a focus on second harmonic generation (SHG) used in the novel bandwidth compression technique for Raman pump generation presented in the following chapter 3.

- Thirdly, the general concept and method of femtosecond stimulated Raman spectroscopy is presented, including an introduction to the spontaneous Raman effect and spontaneous Raman spectroscopy, and the advantages of harnessing the stimulated Raman effect in the FSRS method. 
- Finally we discuss the proposed application of FSRS to the study of eumelanin pigment and its subunits, and how this may bridge the knowledge gap in understanding the vibrational dissipation mechanisms of these intriguing and illusive materials.

\subsection{Ultra-fast spectroscopy}

Ultra-fast spectroscopy encompasses a range of spectroscopic techniques used for studying molecular phenomena that occur on ultra-fast timescales. In order to capture dynamics of atomic and electronic motion which occur on femtosecond $\left(f_{s}\right) \rightarrow$ nanoseconds ( $n s)$ timescales, a "shutter" faster than the event being recorded is required, analogous to a camera shutter used in classical strobe photography. In ultrafast spectroscopy, ultra-short laser pulses with temporal durations on the same order of magnitude as those of the molecular dynamics being studied are used, in effect, as optical shutters ${ }^{53]}$.

These ultra-short pulses are generated from pulsed lasers, where instead of producing a continuous wave $(\mathrm{CW})$ laser beam, a mode-locked laser delivers a train of ultrashort pulses at some repetition rate, with each laser pulse being in phase with the next. We use a $3 W, 3 k H z$ Ti:Sapphire amplifier which produces laser pulses at a central wavelength of $800 \mathrm{~nm}$ with a temporal bandwidth of $\Delta \tau=100 \mathrm{fs}$ (full width at half maximum (FWHM)) and spectral bandwidth of $\Delta \lambda=11 \mathrm{~nm}$ (FWHM). The amplifier is seeded by a $1 \mathrm{~W}, 80 \mathrm{M} \mathrm{Hz}$ Mai Tai oscillator and pumped by a $20 \mathrm{~W}$ Empower laser. Further details of the laser system used here can be found on the Victoria University of Wellington's website ${ }^{59]}$.

Transient absorption spectroscopy (TA) is perhaps the most well known and widely used technique in ultra-fast spectroscopy, used for investigating the electronic excited state mechanisms in materials ${ }^{[3940]}$. Here, two ultra-short laser pulses work in consort - the first pulse, known as the pump, photo-excites the sample and is followed by a second broadband whitelight pulse arriving at some time delay, which probes the sample and is then collected by a spectrometer. The pump laser pulse is mechanically chopped at half the repetition rate of the probe, and each shot is collected as shot $1=$ "pump on" and shot $2=$ "pump off". The signal is then normalised post processing and presented as ("pump on" - "pump off") / "pump off", or " $\Delta T / T$ ", where $T$ is the intensity of the probe's ground state transmission. A positive $\Delta T / T$ signal arises when 
the sample has absorbed the pump, leaving fewer electrons in the ground state, so that more of the probe photons pass through the sample - known as ground state bleach (GSB). A negative $\Delta T / T$ signal arises when the excited state of the sample is populated and further absorbs the probe, leaving fewer photons to pass through the sample and thus presenting as a negative signal, known as photo induced absorption (PIA) [40].

Femtosecond stimulated Raman spectroscopy is effectively an extension of the TA technique where by incorporating a third laser pulse, the vibrational dynamics of the excited state can also be interrogated. The FSRS technique is described in detail in section 2.5 of this chapter. First, an introduction into the phenomenon of nonlinear optics is presented.

\subsection{Nonlinear optics}

Nonlinear optics is important to this work as it describes the processes involved in generating three laser pulses with appropriate spectral and temporal qualities to perform FSRS experiments. Below, the theoretical background of nonlinear processes is introduced, as well as the specific processes involved in the experimental setup of the FSRS system.

Nonlinear optics describes phenomena that occur when the properties of a material system are altered by the presence of light ${ }^{[60+64}$. Pulsed lasers have sufficient intensity to induce optical nonlinearities in mediums with nonlinear susceptibilities, producing fascinating combinations of photonic interactions. The induced polarisation in the nonlinear medium acts as a radiating dipole due to the accelerating charges, which radiates electric fields from contributions of the induced linear and nonlinear polarisations. In linear optics, the induced polarisation $\tilde{P}(t)$ in a medium can be assumed as linear and its magnitude scales linearly with the strength of the incident electric field $\tilde{E}(t)$ as described by

$$
\tilde{P}(t)=\varepsilon_{0} \chi^{(1)} \tilde{E}(t)
$$

where $\chi^{(1)}$ is the linear susceptibility of the medium, and $\varepsilon_{0}$ is the permitivity of free space. However, when the incident optical field is of sufficient intensity, higher order 
polarisation terms arise and equation 2.1 can be expanded as a power series revealing second, third (and higher) order polarisation terms scaling nonlinearly with electric field strength . Assuming the polarisation at some time $t$ depends only on the instantaneous electric field (in the slow varying approximation where $\tilde{E}$ varies slowly in time compared to the wavelength period), the new polarisation terms can be described in the second order and third order respectively as

$$
\tilde{P}^{2}(t)=\varepsilon_{0} \chi^{(2)} \tilde{E}^{2}(t)
$$

and,

$$
\tilde{P}^{3}(t)=\varepsilon_{0} \chi^{(3)} \tilde{E}^{3}(t) .
$$

The physical phenomena these equations describe respond in a nonlinear way to the electric field strength, and can be selectively chosen by experimental configuration due to the dependence on the phase matching conditions of the medium. Put simply, a laser pulse of a certain colour, spectral bandwidth and temporal bandwidth can be transmitted through a nonlinear medium to produce a new laser pulse of different spectral and temporal qualities, scaling exponentially in intensity to the input electric field.

Second harmonic generation is a unique case of nonlinear optics, where by combining two optical fields (two pulsed laser beams propagating together) the second harmonic of the propagating laser beams is produced ${ }^{61}$. The combined electric field can be generally described (for sum and/or difference frequency generation) by the addition of the two spectral components as

$$
\tilde{E}(t)=E_{1} \mathrm{e}^{-i \omega_{1} t}+E_{2} \mathrm{e}^{-i \omega_{2} t}+\text { c.c. }
$$

In the case of second harmonic generation, $\omega_{1}$ and $\omega_{2}$ are equal frequencies, and $E_{1}=$ $E_{2}$, so that the input electric field is simply represented as

$$
\tilde{E}(t)=E \mathrm{e}^{-i \omega t}+\text { c.c. }
$$

Assuming that the electric field intensity is sufficiently high to induce second order polarisation terms in the medium, we can use equation 2.2 to describe the induced second order nonlinear polarisation for SHG as

$$
\tilde{P}^{2}(t)=2 \varepsilon_{0} \chi^{(2)} E E^{*}+\left(\varepsilon_{0} \chi^{(2)} E^{2} \mathrm{e}^{-i 2 \omega t}+c . c\right) .
$$


We see from the polarisation terms that the first linear term has no frequency component, but leads to a static electric field across the nonlinear crystal in a process called optical rectification. The second term of the equation, however, has a contribution with a frequency of $2 \omega$, and it is this term that leads to the generation of radiation at the second-harmonic frequency of the input electric field.

Of experimental interest to this work, are the phenomena of second harmonic generation (SHG) ${ }^{\sqrt[61]]{6}}$ and sum frequency generation $(\mathrm{SFG})^{[63]}$ to produce the Raman pump; third harmonic generation (THG) ${ }^{[61]}$ for the generation of the UV excitation pump, and the third order phenomena involved in the various processes involved in white light generation (WLG) ${ }^{[62[65+69]}$. These processes are described in the relevant pulse generation sections of chapter 3 (Raman pump generation) and chapter 4 (UV excitation pump and whitelight probe generation).

\subsection{Chirp}

A useful concept to introduce here is that of laser pulse chirp. In a transform limited laser pulse all the wavelengths, or colours, within the pulse travel together in phase with each-other and the pulse in this ideal scenario will be at its shortest possible duration for a given spectral bandwidth in the limit of the time-bandwidth product, where (for a Gaussian laser pulse) $\Delta \tau \Delta v \approx 0.44^{[70]}$. Here, the broader the pulse in frequency, the shorter the pulse in temporal duration, and vice versa. A chirped laser pulse, however, is one where the wavelengths are instead spread out in terms of their relative phase. A pulse can be chirped either in time (in the temporal domain) or in space (in the frequency domain). In the case of temporal chirp, a positively chirped pulse is one where the lower energy (longer (red)) wavelengths travel with a higher velocity that the higher energy (shorter (blue)) wavelengths, and thus lead the pulse. A negatively temporally chirped pulse is the opposite, where the blue wavelengths lead the red ${ }^{[71]}$. A spatially chirped pulse, on the other hand, has the colours spread out in physical space, as opposed to in time. Figure 2.1 shows the two scenarios of temporal and spatial chirp.

A pulse can become chirped for a variety of reasons, some purposeful and some erroneous. Temporal chirp occurs when different colours travel at different velocities due to the wavelength dependent refractive index of the medium the pulse is propagating 
temporal chirp (time domain)

positive chirp

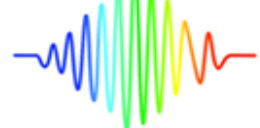

negative chirp

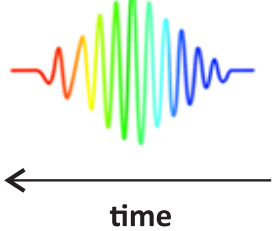

spatial chirp (frequency domain)

positive chirp

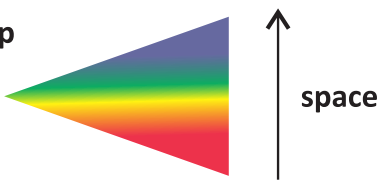

negative chirp

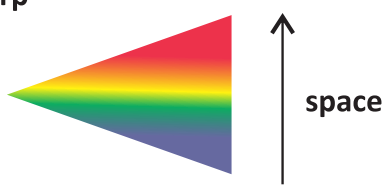

Figure 2.1: The temporal and spatial chirp of a laser pulse. A pulse can be chirped in time (left panel) where the colours are spread out in time after propagation through media where each colour sees a wavelength dependent refractive index. A laser pulse can also be chirped in space (right panel) after being incident on a dispersion element such as a prism or grating.

through. This is especially problematic when a short laser pulse travels through thick transparent optics of high refractive index. For the shortest of pulses, for example, a laser pulse can become temporally chirped just by propagating through air ${ }^{[72]}$. In ultrafast spectroscopy temporal chirp is readily seen in WLG, where the broad spectral range of the propagating supercontinuum means that different colours arrive at the detector at different times ${ }^{[73]}$. This temporal chirp needs to be corrected for in the data processing, to "undo" the velocity differences of each colour and return the pulse to as close to being transform limited as possible ${ }^{[74]}$. Spatial chirp can occur when a laser pulse is incident on a dispersive element, such as a grating, which separates the colours in space, which is useful (and, in fact, essential) in spectroscopic experiments. It is this concept of spatial chirp that is capitalised on in the development of a novel method for Raman pump generation, detailed in the subsequent chapter 3.

\subsection{Femtosecond stimulated Raman spectroscopy}

The spontaneous Raman effect is the phenomenon of inelastic light scattering, first observed by C.V. Raman in $1928^{[7576}$. When incident photons of energy $h \nu_{\text {in }}$ couple to a vibrational Raman-active mode of a molecule of energy $h \nu_{v i b}$ they are in-elastically scattered at a lower (Stokes-shifted) frequency $h \nu_{\text {stokes }}=h \nu_{i n}-h \nu_{v i b}$, or a higher (anti- 
Stokes shifted) frequency $h \nu_{\text {stokes }}=h \nu_{\text {in }}+h \nu_{v i b}{ }^{\text {[75]76]. }}$.

Spontaneous Raman spectroscopy is a spectroscopic tool that allows observation of photon energies in-elastically scattered from materials [77]. These frequencies present as peaks on a spectrograph, and are unique to each molecular structure, allowing materials to be effectively "fingerprinted". In spontaneous Raman spectroscopy, a monochromatic laser source illuminates the sample of interest, and frequency-shifted photons are collected on a spectrograph to produce a Raman spectrum. The measured energy shifts correspond to nuclear vibrations of the molecule, and thus detailed structural information about the molecule can be obtained ${ }^{[7778]}$.

Raman scattering is a notoriously weak process (the number of Raman photons scattered $=10^{-8} \mathrm{x}$ the number of incident photons) and as photons scatter in a dipole emission radiation pattern, strong fluorescent backgrounds which also emit in this pattern can easily overwhelm weak Raman signals.

Stimulated Raman spectroscopy overcomes the challenges in spontaneous Raman spectroscopy by taking advantage of the stimulated Raman process shown in figure 2.2. Stimulated Raman gain, first observed in 1962 by Woodbury and $\mathbf{~ N g}$, overcomes challenges in measuring spontaneous Raman scattering signals by the use of a pumpprobe laser pair to stimulate Raman photon emission, producing photon yields up to $10 \%$ higher than in spontaneous Raman scattering. The emitted photons are in phase and directionally coincident with the direction of the measured probe pulse, meaning the relative contribution from any fluorescent background is minimised ${ }^{[79-81]}$. In stimulated Raman spectroscopy, a monochromatic laser pump works in consort with a probe laser pulse on a sample. When the difference in frequency between the pump and probe photons matches a specific vibrational quantum of the system, a vibrational coherence is induced, stimulating the emission of photons at the corresponding Stokes (or anti-Stokes) shifted frequency ${ }^{82[83]}$. This technique is well established for measuring ground-state vibrational fingerprints of molecules, however, due to the fundamental coupling of energy and time in a laser pulse, achieving both high spectral and temporal resolution to reveal excited state dynamics is limited, as ultrashort pulses in the time domain lead to spectrally broad pulses in the frequency domain [53].

In 2003, Mathies and coworkers found a solution to this problem with the development of femtosecond stimulated Raman spectroscopy (FSRS), which effectively decouples time and frequency resolution by using a sequenced combination of laser pulses, 
stimulated Raman process (Stokes-shifted)

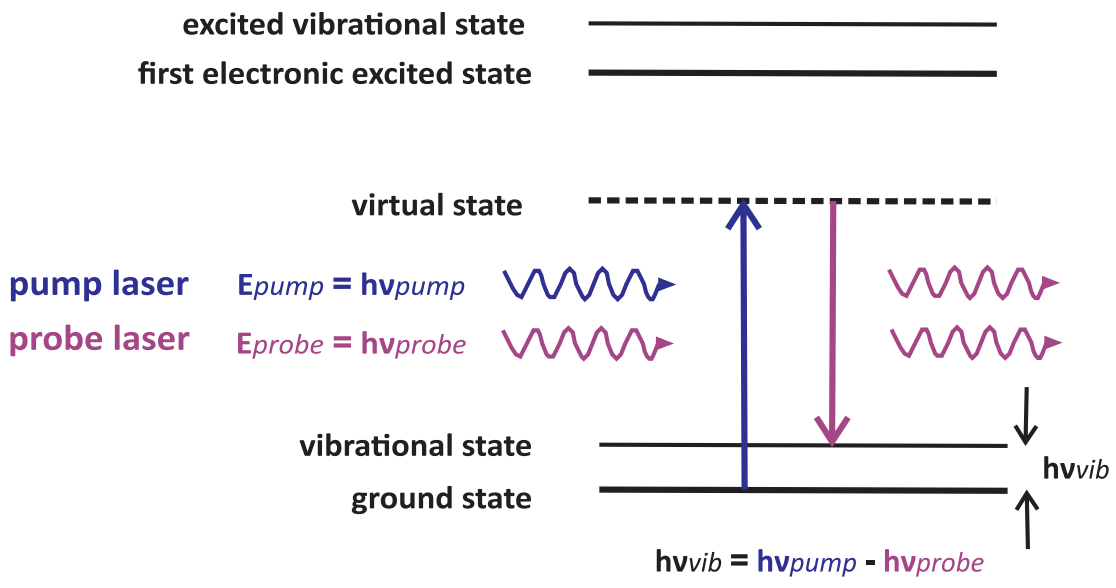

Figure 2.2: The stimulated Raman process (Stokes-shifted) takes advantage of vibrational coherence between energy states. Two laser pulses are incident on a material, with energies $h v_{\text {pump }}$ and $h v_{\text {probe }}$. When the difference in energy between these photons matches a vibrational state of the material a new photon is produced at the energy of the probe. The new photon of energy $h v_{v i b}$ is in phase and co-directional with the probe photon.

while maintaining a high signal-to-noise ratio (SNR) ${ }^{[54-58|84| 85]}$.

Femtosecond stimulated Raman spectroscopy is a 3 laser pulse, 4 wave nonlinear mixing technique whereby an ultrashort femtosecond $(f s)$ excitation laser pulse initiates the photochemistry of interest - in this case the absorption of a UV or visible photon. At some time $\Delta \tau$ later, an intense narrowband Raman pump and a broadband supercontinuum probe laser pair arrive together at the sample. When the energy difference between the Raman pump and probe pulses match an excited state vibrational mode of the system, a vibrational coherence is induced across the molecule at that frequency as shown in figure 2.3 .

While the temporally long Raman pump exists in the sample, subsequent pump photons interact with this coherent oscillation, and stimulate the emission of photons at the corresponding Stokes (or anti-Stokes) shifted frequency travelling in phase and directionally coincident with the probe pulse. 
femtosecond stimulated Raman process
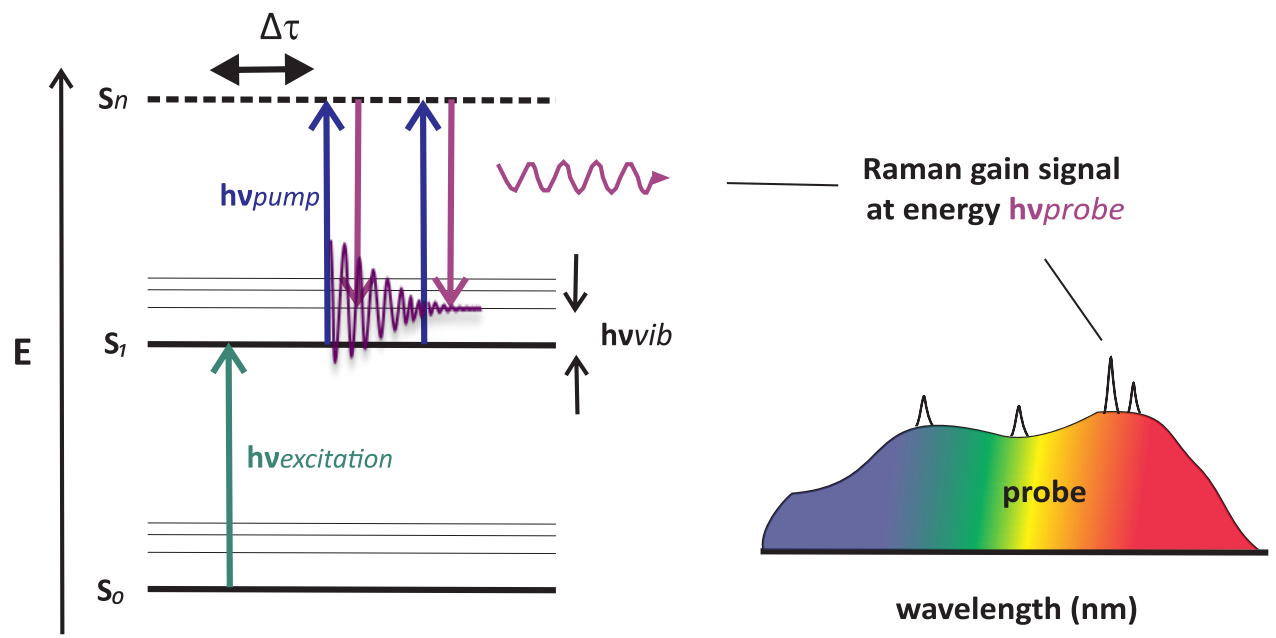

Figure 2.3: Energy level description of the 3 pulse, 4 wave mixing process of femtosecond stimulated Raman spectroscopy. Here an excitation pump of some energy $h v_{\text {excitation }}$ (shown as the green line) photo-excited the sample into a higher energy electronic state. At some time delay, $\Delta \tau$ a second pump arrives (the Raman pump) with an energy $h v_{\text {pump }}$, shown at the blue line. At the same time the probe pulse arrives, shown as the purple line, and together they create a vibrational coherence of the excited state vibrational modes. As this coherent envelope beats, photons are emitted at the difference of their two energies, $h v_{v i b}$. The emitted photon is in phase and co-directional with the probe pulse and becomes a heterodyned signal riding on the probe pulse. Because the probe pulse is broadband, all vibrational modes of the excited state a coherently excited together. In the schematic on the right, an example of 4 vibrational energies, shown as peaks on the probe envelope, have been stimulated in the excited state. By delaying the arrival time $\Delta \tau$ between the excitation pump and Raman pump/probe pair, dynamic vibrational information on $f s$ $n s$ timescales of the excited state can be observed.

Stokes-shifted photons are recorded as a heterodyned Raman gain signal on the probe pulse spectral envelope using a high resolution spectrograph. Scanning the time delay between photoexcitation and the arrival of the pump-probe pair allows Raman spectra to be captured at $f s$ time steps throughout the vibrational dephasing time of the system. The intensity, frequency and bandwidth of the Raman gain peaks are modelled 
as a function of time, revealing the structural evolution of the molecule throughout its vibrational relaxation ${ }^{54}[56$.

The time resolution of the system is the cross correlation of the excitation pump and supercontinuum probe temporal bandwidths, while spectral resolution is given by the shorter of either the Raman pump spectral bandwidth, or the Fourier transform of the inherent molecular dephasing time. The Raman gain is measured according to the equation

$$
\text { Raman gain }=\exp \left[\alpha \cdot \sigma_{R} c I \rho_{\mu} z\right] \text {, }
$$

where $\alpha$ is a sample dependent constant in $\mathrm{cm}^{2} s, \sigma_{R}$ is the Raman scattering cross section of the sample, $c$ is the sample concentration in $m o l / L, I \rho_{\mu}$ is the Raman pump intensity (power/unit area) and $z$ is the sample length $(m)^{[57]}$. In stimulated Raman spectroscopy, the Raman gain is a unit-less measure of the ratio $I_{\text {out }} / I_{i n}$, where $I_{\text {in }}$ is the intensity of the incident probe photon field at the sample, and $I_{\text {out }}$ is the intensity of the transmitted probe photon field ${ }^{[57]}$.

\section{Application to eumelanin pigment}

Where the knowledge gap exists in eumelanin, is in the exact arrangement of eumelanin's chromophores, and more specifically, how absorbed energy is dissipated on such rapid timescales. The radiative emission of eumelanin is $<0.01 \% \sqrt{38}$, indicating rapid non-radiative relaxation upon exposure to light. Using FSRS, capable of resolving vibrational dynamics on $f s$ to $n s$ timescales, we aim to reveal the non-radiative relaxation pathways of eumelanin upon exposure to light. To apply this method to eumelanin, an excited state population is generated using an excitation pump in the $\mathrm{UV}$ and visible regions, where eumelanin strongly absorbs. The Raman pump is set to be as close as possible to the photo-induced absorption (PIA) band in order to achieve resonance enhancement of the excited state Raman gain signals ${ }^{866+91]}$.

Interrogating eumelanin's excited state vibrational manifold (and its subunit monomeric building blocks) using excitation wavelengths in the UV and visible regions, allows comparisons to be made with its monomeric building blocks DHI and DHICA, whose optical properties and vibrational dynamics are bench-marked in chapter 5. 


\subsection{Chapter summary}

In this chapter the concept and background of the Raman effect and the FSRS technique is introduced. A background into nonlinear optics - fundamental to the implementation of the FSRS system - is also presented.

The following two chapters focus on the method of implementing the FSRS system in its application to eumelanin. First, the experimental details of generating an intense, narrowband Raman pump, using both novel and classical methods are presented in chapter 3. In the following chapter 4 , the full FSRS build is presented, including details of the generation of the three laser pulses required; the FSRS system design and layout, including the high resolution spectrographs, and data collection and processing methods used to extract the dynamic FSRS excited state signatures from eumelanin and its subunits. 
Page 34 


\section{Chapter 3}

\section{A novel method to generate a narrowband Raman pump}

This chapter contains elements from our published work:

Efficient and tunable spectral compression using frequency-domain nonlinear optics K E Thorn, N R Monahan, S K K Prasad, K Chen, J M Hodgkiss

Opt Express. 2018 Oct 15;26(21):28140-28149. PMID: 30469869 doi: 10.1364/OE.26.028140. 92$]$

\subsection{Introduction}

In this chapter we present two methods for generating a narrowband, intense Raman pump, as introduced in chapter 2. It is important in ultrafast vibrational spectroscopy to have a tunable Raman pump in order to achieve resonance with a material's PIA band, thus improving the Raman gain signal response by up to 100 times ${ }^{\text {[93. }}$. Here we first outline a novel method for the generation of a Raman pump using frequency domain nonlinear optics, specifically for second harmonic generation. Secondly, the well known spatial filtering method is used to generate a Raman pump in the IR.

\subsection{Raman pump generation using frequency-domain non- linear optics}

A key requirement in the field of ultrafast vibrational spectroscopy is to efficiently generate intense, tunable and narrowband picosecond laser pulses synchronised to a broadband femtosecond laser source. Current nonlinear methods for picosecond pulse generation suffer from complexities in both experimental implementation and pulse 
frequency tunability. We present here a straightforward method for spectral bandwidth compression that produces frequency tunable picosecond pulses with efficient power conversion, which successfully addresses these challenges. Broadband femtosecond laser pulses are spectrally compressed to narrowband picosecond pulses using frequency domain SFG of spatially chirped pulses, achieving spectral bandwidths of $<20 \mathrm{~cm}^{-1}$ and a power conversion efficiency of $\sim 18 \%$. The experimental design of the bandwidth compressor is presented and its application to stimulated Raman spectroscopy is demonstrated.

In this chapter, we first discuss the method background, then present details of our experimental design and demonstrate efficient generation of pulses that are sufficiently narrow for vibrational spectroscopy applications and widely tunable. Finally, we present stimulated Raman spectroscopy measurements for solvents as a demonstration of one potential application for our new bandwidth compression technique.

\subsection{Important aspects of the Raman pump}

\subsubsection{Optical properties}

Arguably the most important aspect in achieving a high SNR in stimulated Raman spectroscopy are the optical properties of the Raman pump. The key elements of interest are the pump's intensity and temporal profile, and experimentally, the beam's crossing angle into the sample.

The intensity of the pump is directly proportional to the Raman gain, while an ideally Gaussian temporal profile maximises the interaction with the probe in the sample. Similarly, a small crossing angle into the sample allows the pump to propagate through the material at a maximum pathlength. The Raman gain equation, given by equation 2.7 of the previous chapter, shows important elements in addition to Raman pump intensity, $I \rho_{\mu}$, and pathlength propagation, $Z$, as the Raman scattering cross section of the material, $\sigma_{R}$, the sample concentration, $c$, and sample dependent constant $\alpha^{[57]}$. In this section we focus on the generation of a highly intense Raman pump to maximise $I \rho_{\mu}$, while having an ideally Gaussian temporal profile. Experimentally, the crossing angle into the sample is minimised to maximise the pathlength interaction of the pump. This is discussed in more detail in section 4.3.2. 


\subsubsection{Limitations of existing methods}

Generation of intense narrowband picosecond laser pulses is of significant interest in ultrafast laser applications where high spectral resolution is required, such as femtosecond stimulated Raman spectroscopy (FSRS) ${ }^{[568494 \mid 96]}$ and sum-frequency generation (SFG) surface spectroscopy ${ }^{197}$. More recently spectral compression of single photons for quantum information transfer has been demonstrated using nonlinear SFG in the time domain ${ }^{[98}$. However, spectrally compressing broadband femtosecond laser pulses to produce synchronised narrowband picosecond pulses introduces severe trade-offs between achievable bandwidth compression, power conversion efficiency, frequency tunability and experimental simplicity.

Linear methods of bandwidth compression involve spectrally filtering a femtosecond broadband laser source, typically in a folded $4 \mathrm{f}$ grating filter configuration, to produce broadly tunable narrowband picosecond pulses. While experimentally straightforward, spectral filtering methods have inherently low power conversion, with throughput of about $\sim 0.3 \%$ for a typical spectroscopy configuration ${ }^{[5799]}$. Other spectral compression methods suffering limitations due to low efficiency include self-phase modulation in optical fibre 100101$]$. A more efficient method of spectral compression exploits the nonlinear phenomenon of sum-frequency generation (SFG) in the time-domain. In this technique two femtosecond pulses at a central frequency $\omega_{F F}$ are equally and oppositely chirped in time and mixed in a suitably phase-matched nonlinear crystal to produce a narrowband picosecond pulse at the second harmonic frequency $\omega_{S H}$ [1021103]. Methods to generate temporally chirped pulses for nonlinear mixing include gratingstretcher pairs ${ }^{[102-104]}$, and chirped volume Bragg gratings ${ }^{[105]}$. While successfully addressing conversion efficiency, time-domain nonlinear bandwidth compression techniques, including SFG and the related difference-frequency generation (DFG) ${ }^{1061107}$, are generally experimentally complex to tune, optimise and characterise and require expensive optical components.

\subsection{Method background}

\subsubsection{Sum frequency generation}

Sum frequency generation (SFG) is a well described phenomenon whereby two photons of frequencies $\omega_{1}$ and $\omega_{2}$ and wave vectors $k_{1}$ and $k_{2}$ propagate together in a non- 
linear medium producing a photon at the sum of their combined frequencies $\omega_{3}=\omega_{1}$ $+\omega_{2}$ [108]. Figure 3.1 shows a special case of SFG where photons of differing energies symmetric about a central frequency $\omega_{F F}$ by amount $\pm \Delta \omega$ combine together to generate signal photons at a constant second harmonic frequency $\omega_{S H}$ [63641108109].
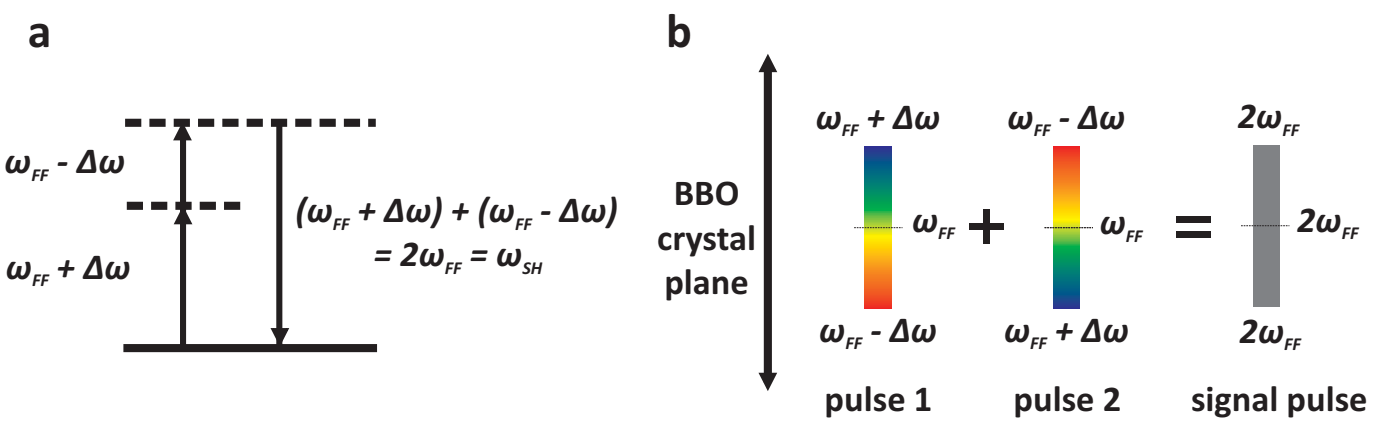

Figure 3.1: (a) SFG of photons with equal and opposite energies $\pm \Delta \omega$ about a central frequency $\omega_{F F}$ combine to produce the second harmonic $\omega_{S H}=2 \omega_{F F}$. Dashed lines represent virtual energy levels. (b) Two pulses of equal and opposite spatial chirp mix together so that all photon combinations produce a narrowband signal pulse at the second harmonic. This figure is reprinted with permission from reference ${ }^{[2]}$ (c) The Optical Society.

Our method takes advantage of this phenomenon, where spectral components of energies $\omega+\Delta \omega$ and $\omega-\Delta \omega$ mix together in the frequency domain to produce a compressed spectral bandwidth at the second harmonic. The method to generate the spatially chirped pulses uses simple mirror imaging following pulse dispersion, which naturally produces two pulses of perfectly opposite chirp symmetry. The signal bandwidth $\Delta \lambda_{S H}$ is proportional to the spectral bandwidths of the individual frequency components at the Fourier plane and can easily be deduced by the geometric configuration of the system. The compressed bandwidth can be predicted using equation (3.1),

$$
\Delta \lambda_{S H} \approx \frac{1}{4} \Delta \lambda_{F F}=\frac{1}{4}\left(\Delta \lambda_{F P}+\Delta \lambda_{D}\right),
$$

where $\Delta \lambda_{F P}$ is the spectral resolution of each colour in the Fourier plane described by equation (3.2),

$$
\Delta \lambda_{F P}=\Delta x_{0} \frac{\delta \lambda}{\delta x},
$$

and $\Delta \lambda_{D}$ is a distortion factor due to imperfect mixing of colours caused by the noncollinear crossing geometry described by Eq. 3.3., 


$$
\Delta \lambda_{D}=L \tan (\alpha) \frac{\delta \lambda}{\delta x}
$$

Here, $\Delta x_{0}$ is the focused Gaussian beam waist, $\delta \lambda / \delta x$ is the linear dispersion at the Fourier plane for a nonlinear crystal of length $L$, and a given input beam angle, $\alpha$. The bandwidth compression of our system configuration is determined by the input wavelength $\lambda_{F F}$, input spectral bandwidth $\Delta \lambda_{F F}$, angular dispersion $\delta \lambda / \delta \theta$ of the chosen dispersion element, lens focal length $f$, input beam diameter $D$, and nonlinear crystal input angle $\alpha$ - which are all factors easily characterised ${ }^{[110-112]}$. The use of a single dispersion element allows for a compact optical arrangement, and signal tuning over a broad frequency range is achieved with simple system adjustments. The derivation of the above equations are presented in the following section 3.4 .2 .

\subsubsection{Bandwidth compression model}

The output signal spectral bandwidth $\Delta \lambda_{S H}$ can be modelled using Gaussian beam optics for a given dispersion element of dispersion, $\Phi$, angle of input to the nonlinear crystal, $\alpha$, crystal length, $L$, focal length of the focusing element, $f$, and the input beam diameter, $D$. The following sections show the derivation of the bandwidth prediction model given by equation (3.1). The derivation of equations (3.2) and (3.3) for the prism configuration is given in 3.4.2.1. The derivation of equations (3.2) and (3.3) for the grating configuration is given elsewhere ${ }^{[103]}$. Section 3.4.2.2 shows a graphical simulation of spectral bandwidths using equation (3.1) for a variety of different model parameters for both the prism and grating configurations.

\subsubsection{Derivation for prism configuration}

For a Gaussian beam of diameter $D$ focused by a lens where $f \gg D$ arranged in a $2 f$ configuration as shown in figure $3.2 a^{[112[113]}$. The beam waist $\left(1 / \mathrm{e}^{2}\right.$ diameter $)$ at the focal plane is:

$$
2 W_{0}=\left(\frac{4 \lambda}{\pi}\right) \times\left(\frac{f}{D}\right)
$$

Converting the beam waist, $2 W_{0}$, to spatial dimensions, the spatial width (in $m$ ), $\Delta x_{0}$, at the focal plane for one colour is given as

$$
\Delta x_{0}=2 W_{0} \sqrt{2 \ln 2}
$$




\section{a foucsing of a Gaussian beam}

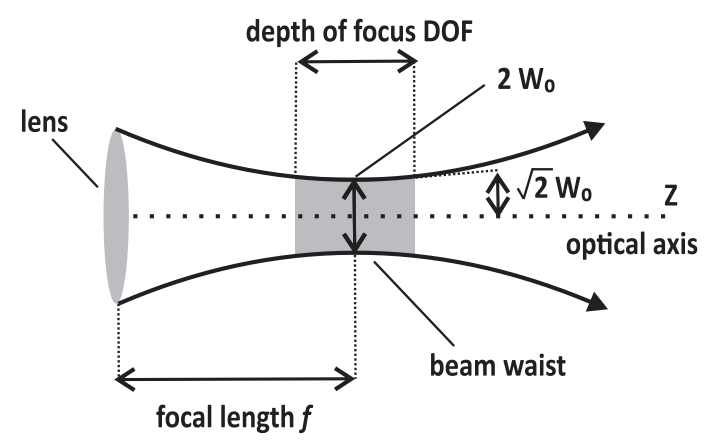

b non-collinear beam geometry

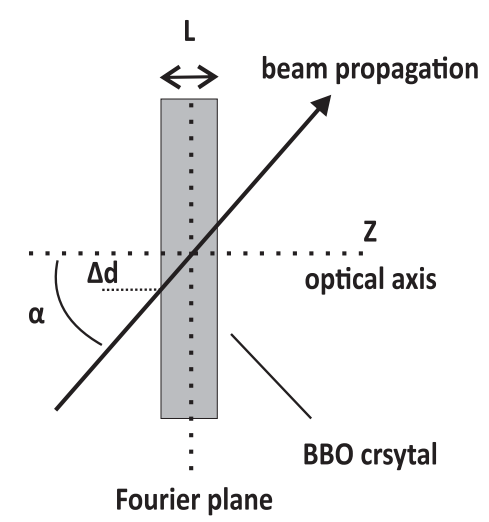

Figure 3.2: Geometric configurations for (a) the focusing of a Gaussian beam in the Fourier plane; and (b) the non-collinear geometry of a beam propagating through a nonlinear crystal.

The beam's depth of focus (DOF) is shown in figure $3.2 \mathrm{a}$, and is calculated from the focal length of the lens, $f$, the beam diameter at the lens, $D$, and the input wavelength, $\lambda$, as

$$
\mathrm{DOF}=\left(\frac{4 \lambda}{\pi}\right) \times\left(\frac{f}{D}\right)^{2} .
$$

The beam diameter illuminated at the lens, $D$, is subject to beam anamorphic magnification, $M$, dependent on the input beam diameter to the prism, $D_{I N}$, prism geometry and input angle to the prism, $\theta_{\mathrm{i} 1}$ as

$$
D=M D_{I N}
$$

where $M$ is the anamorphic prism magnification given by incident and transmitted beam angles calculated using Snell's Law for a beam propagating through a prism as

$$
M=\left(\frac{\cos \theta_{\mathrm{t} 1}}{\cos \theta_{\mathrm{i} 1}}\right) \times\left(\frac{\cos \theta_{\mathrm{t} 2}}{\cos \theta_{\mathrm{i} 2}}\right),
$$

where $\theta_{\mathrm{i} 1}$ is the incident angle and $\theta_{\mathrm{t} 1}$ is the transmitted angle of the beam at the first surface of the prism, and $\theta_{\mathrm{i} 2}$ and $\theta_{\mathrm{t} 2}$ are defined in the same way for the second surface ${ }^{[114}$. 
a spectral resolution of each focused colour

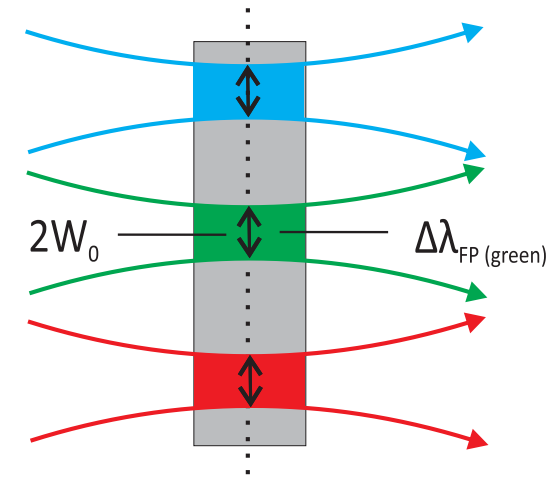

Fourier plane b

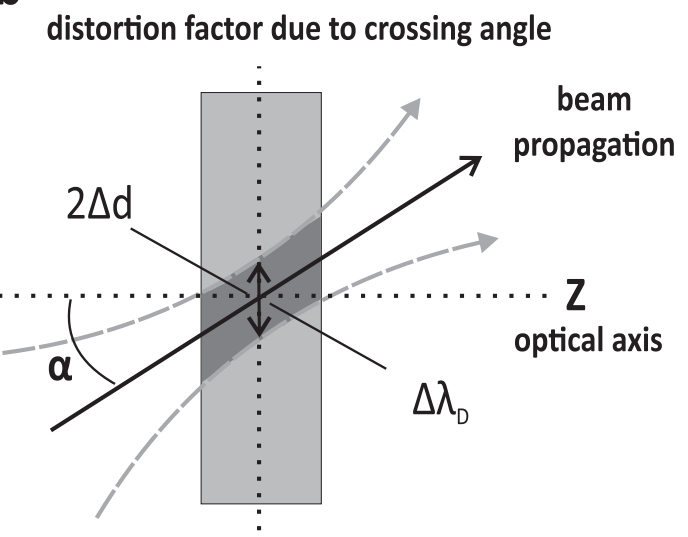

Fourier plane

Figure 3.3: Geometric configurations for (a) the spectral resolution of each colour at the BBO crystal defined in space as $2 W_{0}$ and in wavelength as $\Delta \lambda_{F P}$, and (b) the distortion factor caused by the crossing angle geometry as the beam propagates through the nonlinear crystal defined in space as $2 \Delta d$ and in wavelength as $\Delta \lambda_{D}$

The frequency resolution, $\Delta \omega_{F P}$, is calculated from the spectral resolution of the colours, $\Delta \lambda_{F P}$, at the focal plane as

$$
\Delta \omega_{F P}=\frac{\omega^{2}}{2 \pi c} \Delta \lambda_{F P}
$$

where, rearranging for the spectral resolution of each colour in the focal plane and using the spatial width of the focused colour, $\Delta x_{0}, \Delta \lambda_{F P}$ becomes,

$$
\Delta \lambda_{F P}=\Delta x_{0} \frac{\delta \lambda}{\delta x}
$$

The linear dispersion, $\delta \lambda / \delta x$, is calculated from the angular dispersion $\delta \lambda / \delta \theta$ using the spectral bandwidth, $\Delta \lambda_{F F}$, of the fundamental input pulse, strip width, $X$, at the Fourier plane, for a dispersion cone angle $\Phi$, and lens focal length $f$ as

$$
\frac{\delta \lambda}{\delta x}=\frac{\Delta \theta}{\Delta \lambda} \frac{\delta \lambda}{\delta \theta}=\frac{\Phi \Delta \lambda_{F F}}{2 f \tan \left(\frac{\Phi}{2}\right) \Phi}=\frac{\Delta \lambda_{F F}}{X} .
$$

The compressed spectral bandwidth, $\Delta \lambda_{S H}$, is predicted by the spectral resolution of each frequency at the focal plane, plus broadening due the non-collinear beam input geometry, as shown in figure 3.2(b), and can be approximated as 


$$
\Delta \lambda_{S H} \approx \frac{1}{4} \Delta \lambda_{F F}=\frac{1}{4}\left(\Delta \lambda_{F P}+\Delta \lambda_{D}\right) .
$$

Figure 3.3a shows a schematic of the spectral resolution for each colour in the Fourier plane, where, as in equation 3.10 .

$$
\Delta \lambda_{F P}=\Delta x_{0} \frac{\delta \lambda}{\delta x} .
$$

The distortion factor, $\Delta \lambda_{D}$, due to the crossing angle is shown in figure $3.3 \mathrm{p}$, and can be calculated as

$$
\Delta \lambda_{D}=2 \Delta d \frac{\delta \lambda}{\delta x}=L \tan (\alpha) \frac{\delta \lambda}{\delta x}
$$

Entering in all the defined variables derived above for the focal plane resolution, $\Delta \lambda_{F P}$, and the distortion factor, $\Delta \lambda_{D}, \Delta \lambda_{F F}$ can be first expanded as

$$
\begin{aligned}
\Delta \lambda_{F F} & =\Delta x_{0} \frac{\delta \lambda}{\delta x}+L \tan (\alpha) \frac{\delta \lambda}{\delta x} \\
& =\left(\Delta x_{0}+L \tan (\alpha)\right) \frac{\delta \lambda}{\delta x} \\
& =\left(\frac{4 \lambda f \sqrt{2 \ln 2}}{\pi \mathrm{D}}+L \tan (\alpha)\right) \frac{\delta \lambda}{\delta x} \\
& =\left(\frac{4 \lambda f \sqrt{2 \ln 2}}{\pi \mathrm{D}}+L \tan (\alpha)\right) \frac{\Delta \lambda_{F F}}{2 f \tan \left(\frac{\Phi}{2}\right)}
\end{aligned}
$$

And finally, equation 3.12 for predicting the spectral bandwidth of the signal pulse, $\Delta \lambda_{S H}$, can then be written as

$$
\Delta \lambda_{S H}=\left(\frac{4 \lambda f \sqrt{2 \ln 2}}{\pi \mathrm{D}}+L \tan (\alpha)\right) \frac{\Delta \lambda_{F F}}{8 f \tan \left(\frac{\Phi}{2}\right)} .
$$

The temporal bandwidth of the compressed signal pulse can be predicted from the compressed bandwidth $\Delta \lambda_{S H}$, assuming a transform limited pulse of Gaussian profile where $\Delta \tau \Delta v \approx 0.44^{[70}$. Using the conversion of wavelength bandwidth to frequency bandwidth, where $\Delta v=\Delta \lambda \cdot c / \lambda^{2}$, we can derive the temporal bandwidth as

$$
\Delta \tau_{S H} \approx \frac{0.44}{c} \cdot \frac{\lambda_{S H}^{2}}{\Delta \lambda_{S H}},
$$


where $\lambda_{S H}$ is the central wavelength of the compressed signal pulse, $\Delta \lambda_{S H}$ is the compressed spectral bandwidth and $c$ is the speed of light. Equations 3.15 and 3.16 are used in the following section to compare predicated spectral and temporal bandwidths against those measured.

\subsubsection{Bandwidth compression simulation for the prism and grating configura- tions}

Simulations for a range of input wavelengths $(600 \mathrm{~nm}-1400 \mathrm{~nm})$ for both the prism and grating configurations are shown in figure 3.4 using equation 3.7 by variation of the optical parameters with expected effects on the signal spectral bandwidth $\Delta \lambda_{S H}$. Specifically, the bandwidth, $\Delta \lambda_{S H}$, will favourably decrease for increasing dispersion $\Phi$, beam diameter, $D$, and focal length, $f$ of the system. It will also decrease with a decreasing input angle of the two beams into the BBO crystal, $\alpha$, which allows for "tighter" colour mixing reducing broadening distortions. A negative impact that will broaden the signal bandwidth is an increase in the BBO crystal length, $L$, however this may also increase power conversion.

The bandwidths predicted in section 3.4 .2 for the prism configuration are using an input wavelength of $800 \mathrm{~nm}$ with $\alpha=4.5^{\circ}, X=640 \mu \mathrm{m}$ and $D_{I N}=10 \mathrm{~mm}$. The bandwidths predicted for the grating configuration are using an input wavelength of 800 $n m$ with $\alpha=4.5^{\circ}, X=3.3 \mathrm{~mm}$ and $D_{I N}=6 \mathrm{~mm}$. These figures are given in table 3.1

\begin{tabular}{|l|l|l|}
\hline \multicolumn{3}{|c|}{ Measured and predicted spectral and temporal bandwidths } \\
\hline \hline configuration & measured & predicted \\
\hline prism & $\Delta \lambda_{S H}=0.32 \mathrm{~nm}$ & $\Delta \lambda_{S H}=0.38 \mathrm{~nm}$ \\
& $\Delta \tau_{S H}=0.77 \mathrm{ps}$ & $\Delta \tau_{S H}=0.61 \mathrm{ps}$ \\
\hline \hline grating & $\Delta \lambda_{S H}<0.2 \mathrm{~nm}$ & $\Delta \lambda_{S H}=0.028 \mathrm{~nm}$ \\
& $\Delta \tau_{S H}=10.3 \mathrm{ps}$ & $\Delta \tau_{S H}=10.3 \mathrm{ps}$ \\
\hline \hline
\end{tabular}

Table 3.1: Measured and predicted spectral and temporal bandwidths for the bandwidth compressor in the prism and grating configurations.

Figure 3.4 on the following page shows 4 simulated examples of our model to predict the compressed output signal bandwidth, $\Delta \lambda_{S H}$, for a variety of different variable conditions for both the prism and grating bandwidth compressor configurations. Each simulation was chosen to represent a variety of system parameters that are easily 
changed through the choice of optical elements.
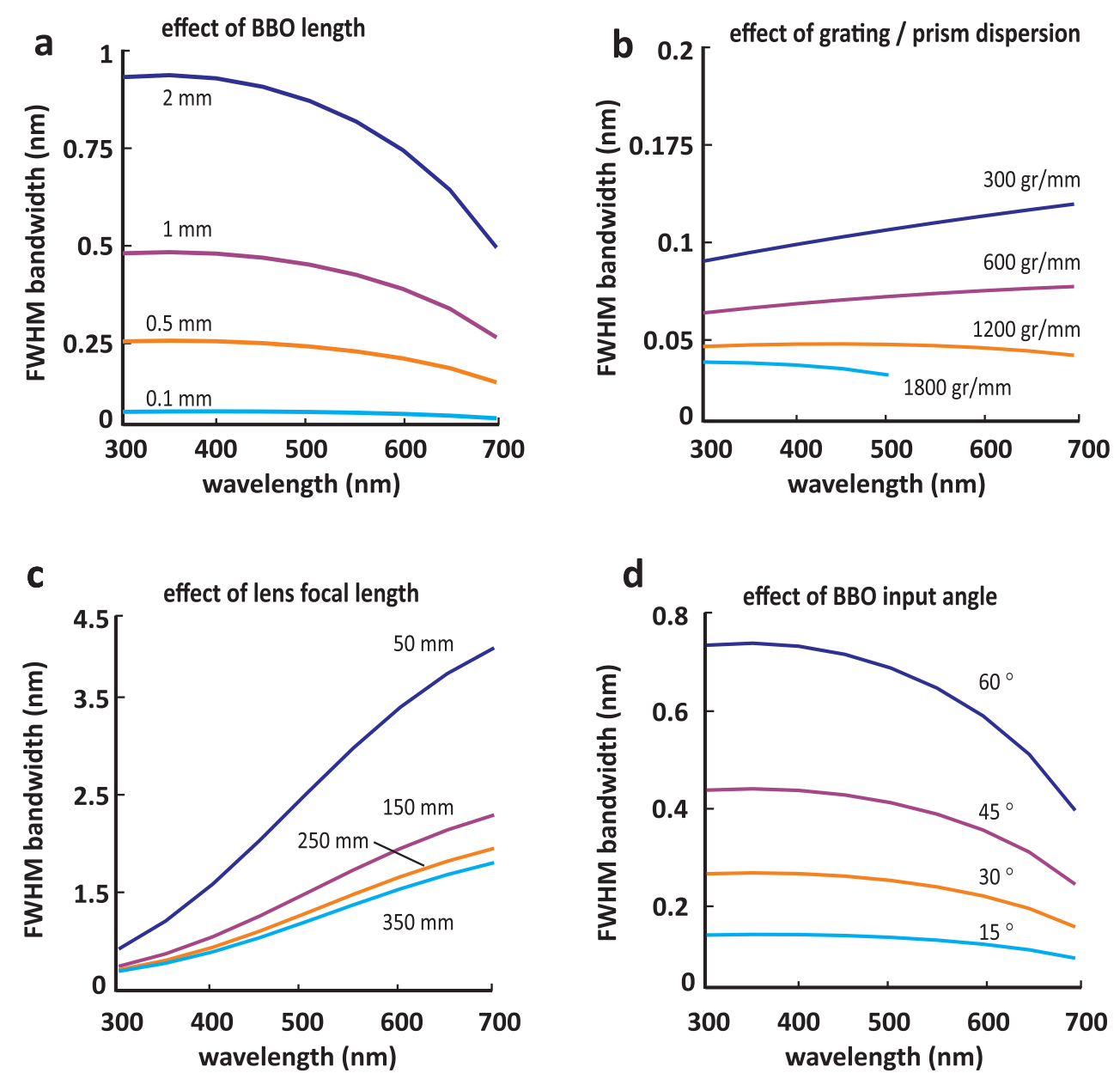

Figure 3.4: Simulated $\Delta \lambda_{S H}$ signal bandwidth predictions for a variety of configurations; (a) the effect of adjusting BBO crystal length bandwidth prediction for the grating configuration with $g=1200 \mathrm{gr} / \mathrm{mm}, D=6 \mathrm{~mm}, \alpha=4.5^{\circ}, f=250 \mathrm{~mm}$; (b) the effect of adjusting dispersion $\Phi$ on bandwidth prediction for the grating configuration with $L=0.5 \mathrm{~mm}, D=6 \mathrm{~mm}, \alpha=4.5^{\circ}, f=250 \mathrm{~mm}$; (c) the effect of adjusting the focal length $f$ on bandwidth prediction for the prism configuration with $L=0.5$ $\mathrm{mm}, D=10 \mathrm{~mm}, \alpha=4.5^{\circ}, L=0.5 \mathrm{~mm}$; and (d) the effect of adjusting the input angle $\alpha$ on bandwidth prediction for the grating configuration with $g=1200 \mathrm{gr} / \mathrm{mm}, L=$ $0.5 \mathrm{~mm}, D=6 \mathrm{~mm}$, and $L=0.5 \mathrm{~mm}$. 


\subsection{Experimental configuration}

\subsubsection{Prism compressor}

To demonstrate our technique, we used a beta barium borate (BBO) crystal of thickness $L=0.5 \mathrm{~mm}$ cut for SHG at $\lambda_{F F}=800 \mathrm{~nm}$, generated from our Ti:Sapphire amplifier producing pulses with FWHM spectral bandwidth $\Delta \lambda_{F F}=11 \mathrm{~nm}$, at a $3 \mathrm{kHz}$ repetition rate. Figure 3.5(a) shows the main components of our experimental layout, configured as an independent and portable system comprising a dispersive element (grating or prism), beam-splitter and mirror pair, focusing lens and BBO crystal arranged in a $2 f$ configuration.

A flint H-ZF62 glass prism with angular dispersion of $6.7 \mathrm{~nm} / \mathrm{mrad}$ (input at Brewster's angle) was chosen as the dispersion element and, in a second set of experiments, a $1200 \mathrm{gr} / \mathrm{mm}$ diffraction grating blazed for $750 \mathrm{~nm}$ with a higher angular dispersion of $0.82 \mathrm{~nm} / \mathrm{mrad}$ (as calculated for input angle $0.95 \mathrm{rad}$ ) replaced the prism. The input pulse energy was $13.3 \mu \mathrm{J}$. Following dispersion, the pulse was split into two copies with a beam splitter (Thorlabs UFBS5050), and two mirrors inverted one pulse with respect to the other, naturally producing two pulses with perfectly symmetric equal and opposite spatial chirp. An achromatic doublet (Thorlabs AC508-250-A-ML) of focal length $f=250 \mathrm{~mm}$ focused the two chirped pulses to the Fourier plane as overlapping strips of length $X=2 f \tan \Phi$, where $\Phi$ is the dispersion cone angle generated by the dispersive element ${ }^{[112]}$.

The two beams were mixed in a non-collinear geometry in the BBO crystal with an input angle of $0.07 \mathrm{rad}$ between them, allowing separation of the signal and fundamental beams with an iris placed at a distance after the BBO crystal. Optimisation of the spatial and temporal overlap of the chirped pulses in the Fourier plane is achieved using the fine adjustment of a single optical element, mirror M1. Rotating the mirror spatially overlaps the focused strips in the perpendicular axis of the BBO crystal, while translating the same mirror along the optical axis allows path length matching to maximise temporal overlap. The signal beam is divergent perpendicular to the mixing axis and is collimated with a cylindrical lens. 
(a)

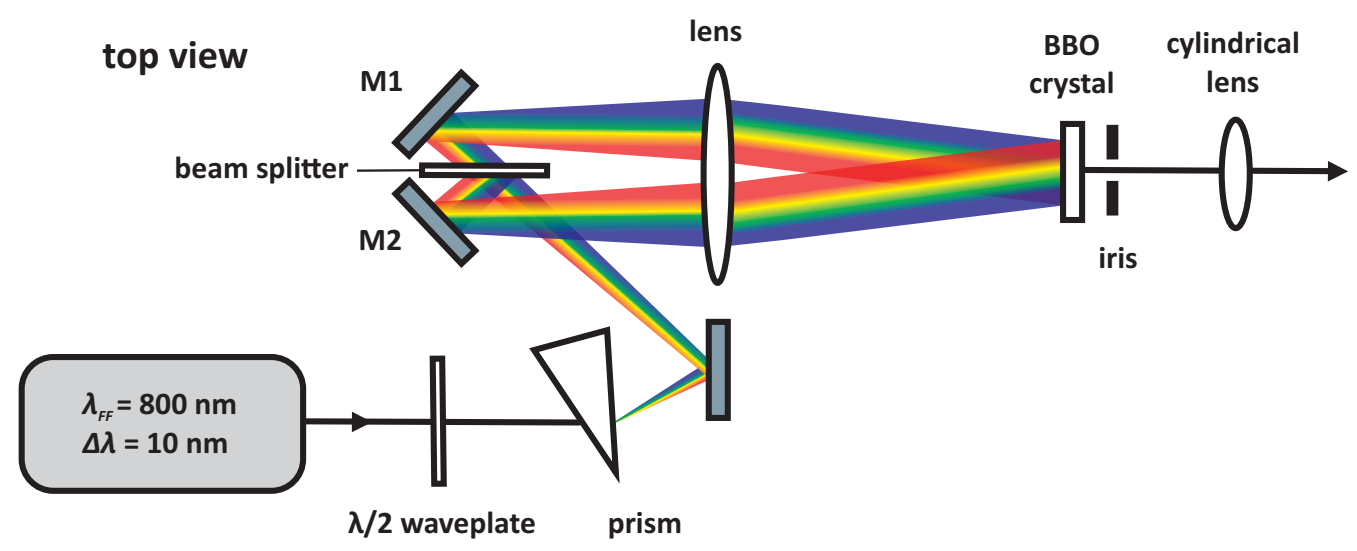

(b)

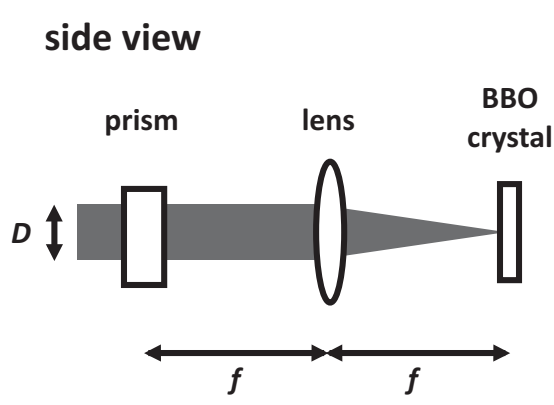

(c)

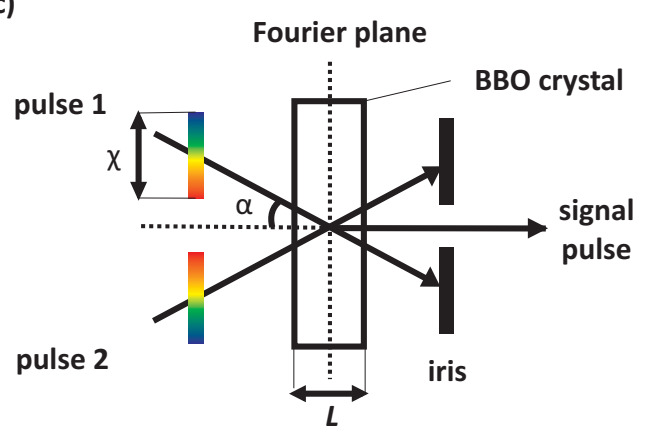

Figure 3.5: (a) Experimental configuration (top view) of the frequency-domain bandwidth compressor where the dispersive element is shown as a prism which can be interchanged with a diffraction grating depending on efficiency and compression requirements; $\mathrm{AL}$, achromatic doublet lens; $\mathrm{BBO}$, type-1 nonlinear crystal of thickness $0.5 \mathrm{~mm}$; CL, cylindrical lens; all other non-marked components are plano silver mirrors. (b) Side view of the bandwidth compressor configured in a $2 f$ geometry; the input beam of diameter $D$ is incident on the prism and focused to a strip at the Fourier plane by the achromatic lens. (c) Non-collinear geometry of the 2 input pulses of strip length $X$ to the BBO crystal of length $L$ with a crossing angle $\alpha$. The iris placed after the crystal blocks the fundamental pulses and the compressed signal pulse propagates along the optical axis of the system. This figure is reprinted with permission from reference [92] (c) The Optical Society.

\subsubsection{Grating compressor - layout improvements}

A gold coated $600 \mathrm{gr} / \mathrm{mm}$ reflective grating blazed for what was set up in an altered configuration. The advantages gained here are a higher throughput in the nonlinear conversion, and much more straight forward alignment. The addition of a polariser also allows separation of the laser pulses with equal intensity through the $50: 50$ beam 
splitter. The technical layout of this configuration is given in appendix A.

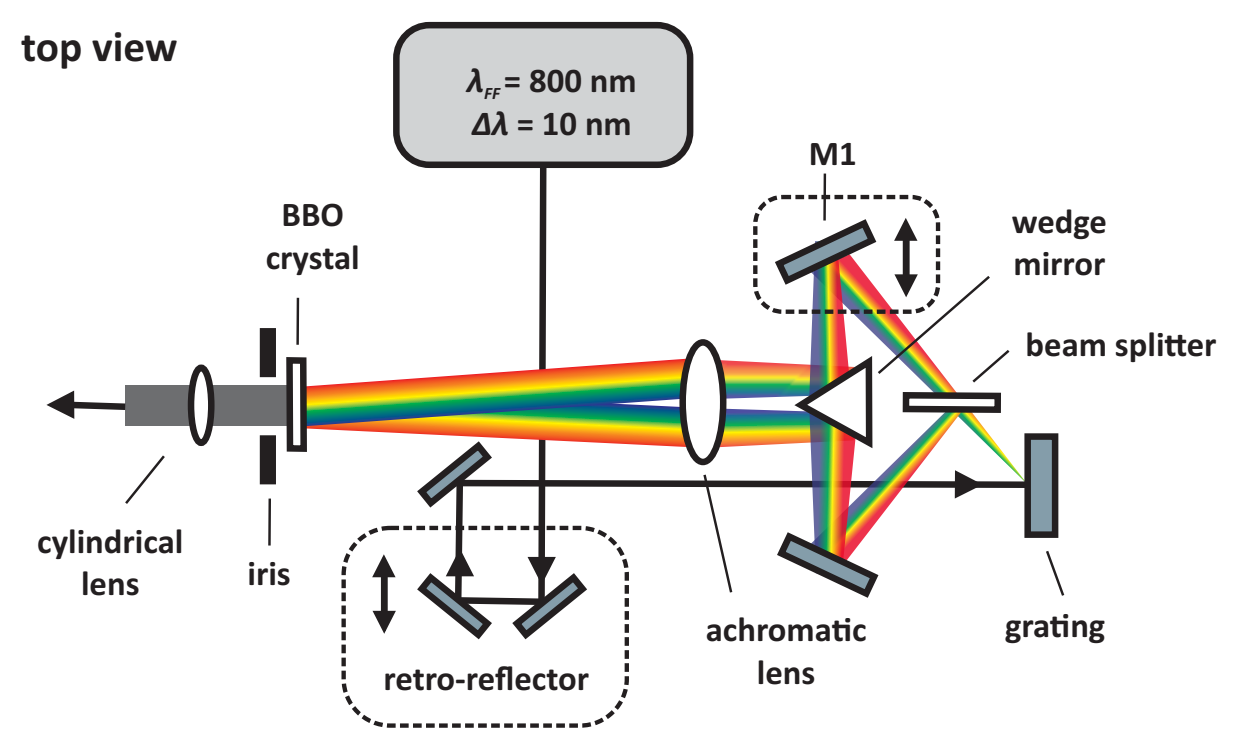

Figure 3.6: Experimental configuration for the improved grating compressor; the fundamental beam is first sent through a retro-reflector for pathlength matching with the probe pulse, the grating disperses the beam which is split by a 50:50 beam splitter and sent to two planar mirrors. M1 is on a translation stage allowing pathlength matching of the two beam paths before the BBO crystal. A wedge mirror allows the beams to be sent to the achromatic lens in a parallel configuration, reducing aberrations caused by any crossing angles input to the lens. The BBO crystal compresses the beams and a cylindrical lens collimates the compressed beam which is then sent to be focused at the sample.

\subsection{Experimental results}

\subsubsection{Spectral compression}

To characterise the spectra of the signal pulse, a fibre-coupled spectrograph (Brolight BIM-6602-02) with optical resolution (OR) of $0.2 \mathrm{~nm}$ recorded the pulse spectra. Figure 3.9 (a) shows the spectra of the signal generated using an H-ZF62 prism and a 1200 $\mathrm{gr} / \mathrm{mm}$ grating as the dispersive elements, demonstrating a significant compression factor of $\sim 9$ of the input pulse, with a measured $\Delta \lambda_{S H}$ of $0.32 \mathrm{~nm}\left(20.0 \mathrm{~cm}^{-1}\right)$ for the prism. This result is close to the predicted spectral bandwidth of $\Delta \lambda_{S H} \approx 0.38 \mathrm{~nm}(23.8$ $\mathrm{cm}^{-1}$ ) determined by equation 3.1 . 

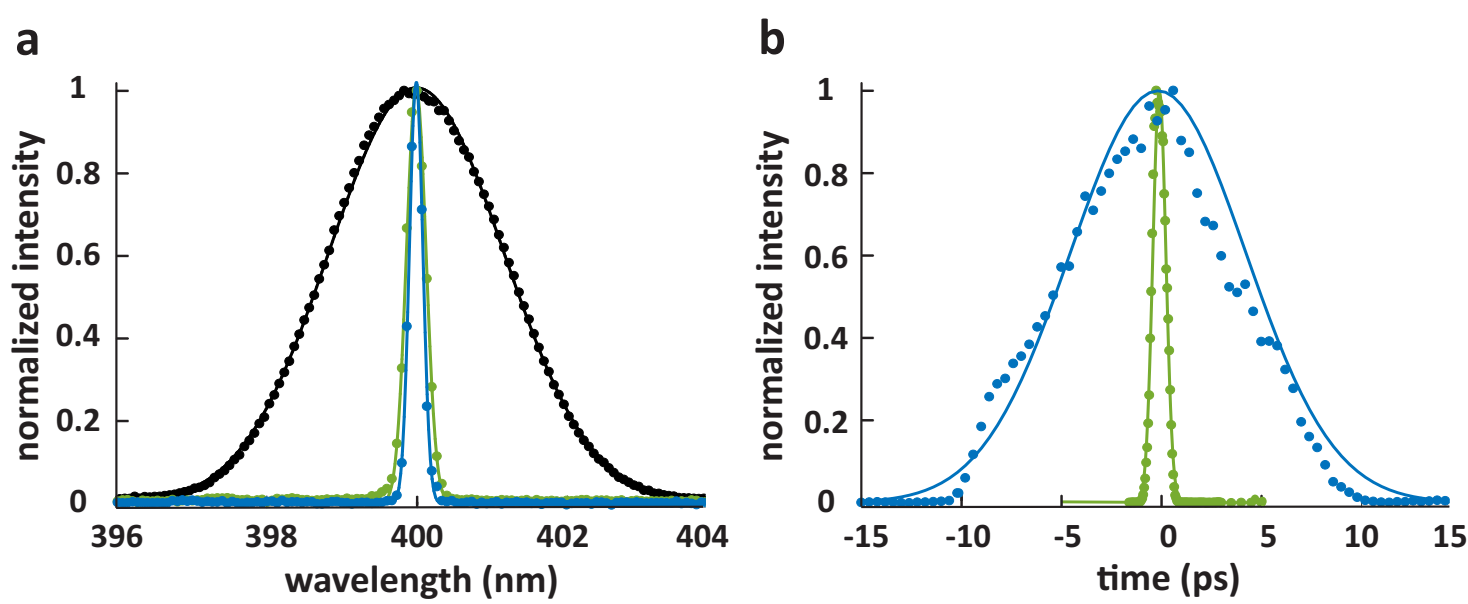

Figure 3.7: (a) Comparison of the second harmonic generation bandwidth of a 400nm pulse (black line), sum-frequency generation of chirped pulses using a prism (green line) a grating (blue) showing a spectral bandwidth $\Delta \lambda_{S H}$ of $2.73 \mathrm{~nm}$, $0.32 \mathrm{~nm}$ and $<0.22 \mathrm{~nm}$ respectively. (b) Temporal profile of the bandwidth compressed $400 \mathrm{~nm}$ signal generated with a prism (green line) and grating (blue line) showing a Gaussian temporal pulse width a $\Delta \tau_{S H}$ of 0.77 ps and 10.34 ps respectively. This figure is reprinted with permission from reference ${ }^{92]}$ (c) The Optical Society.

The higher dispersing $1200 \mathrm{gr} / \mathrm{mm}$ diffraction grating resulted in the spectral compression improving beyond the OR limit of our spectrograph, $0.22 \mathrm{~nm}\left(13.8 \mathrm{~cm}^{-1}\right)$, giving a compression factor of $>12$. The predicted bandwidth for this configuration is at $\Delta \lambda_{S H} \approx 0.028 \mathrm{~nm}\left(1.75 \mathrm{~cm}^{-1}\right)$, a magnitude smaller than our resolution.

For comparison, a second harmonic pulse was generated using the same BBO, but without the use of the compressor producing a bandwidth of $\Delta \lambda_{S H}=2.73 \mathrm{~nm}(170.6$ $\left.\mathrm{cm}^{-1}\right)$. The temporal profile of the signal was resolved using a transient-grating method ${ }^{\text {[71115]. }}$. Results in figure 3.9 (b) show a Gaussian profile with a temporal envelope of $\Delta \tau_{S H}=$ $0.77 \mathrm{ps}$ using the prism dispersive element and a temporal envelope of $\Delta \tau_{S H}=10.34 \mathrm{ps}$ using the grating dispersive element. Assuming transform-limited pulses, predicted spectral bandwidths can be derived from the time bandwidth product for a Gaussian profile $\Delta \nu \Delta \tau=0.44$, predicting a spectral bandwidth of $0.31 \mathrm{~nm}(0.32 \mathrm{~nm}$ measured) for the prism configuration, and $0.02 \mathrm{~nm}\left(1.25 \mathrm{~cm}^{-1}\right)$ for the grating configuration. This is in good agreement with the spectral bandwidths predicted by equation (3.1). This bandwidth, and even the best measured bandwidth of $13.8 \mathrm{~cm}^{-1}$, at the OR limit of our 
spectrograph, is comparable or better than other systems with reported bandwidths of $1 \mathrm{~cm}^{-1}$ to $93 \mathrm{~cm}^{-1}[103104106107$.

\subsubsection{Tunability}

Of significant interest to spectroscopic applications is the ability to produce tunable narrowband picosecond pulses across a wide spectral range. Temporal domain compressor systems typically report some broad tunability about the fundamental system wavelength, for example $720 \mathrm{~nm}$ to $890 \mathrm{~nm}^{[116]}$ and $1000 \mathrm{~nm}$ to $1090 \mathrm{~nm}$ [103].
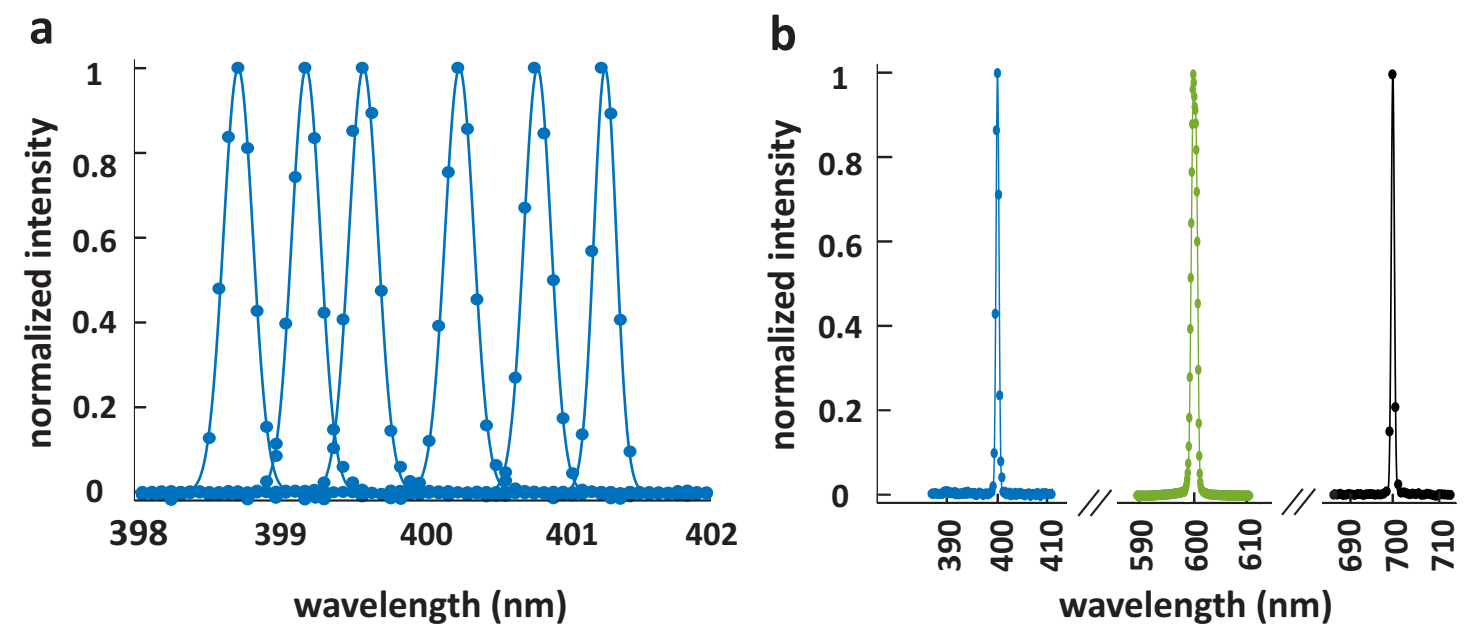

Figure 3.8: (a) Normalised fine tuning capability shown for a range of $\sim 2.5 \mathrm{~nm}$ about central wavelength $\lambda_{S H}=400 \mathrm{~nm}$. (b) Demonstration of signal tuning over a broad frequency range, showing compressed spectra generated using a grating dispersive element, for system input wavelengths $\lambda_{F F}=800 \mathrm{~nm}\left(\lambda_{S H}=400 \mathrm{~nm}\right)$, $\lambda_{F F}=1200 \mathrm{~nm}\left(\lambda_{S H}=600 \mathrm{~nm}\right)$ and $\lambda_{F F}=1400 \mathrm{~nm}\left(\lambda_{S H}=700 \mathrm{~nm}\right)$. This figure is reprinted with permission from reference ${ }^{[92]}$ (C) The Optical Society.

In our system, fine-tuning of the output signal frequency is easily achieved by translating one of the chirped strips with respect to the other in the plane of the BBO crystal using mirror M1. Figure 3.8(a) shows a normalised tuning range of $\sim 2.5 \mathrm{~nm}$ about the central frequency of $400 \mathrm{~nm}$. Broad tuning was demonstrated using two additional input wavelengths generated in an optical parametric amplifier (Light Conversion TOPAS) that was pumped by our $800 \mathrm{~nm}$ fundamental laser. Figure 3.8(b) shows different SFG signal wavelengths generated by adjustment of the input angle to a diffraction grating, and rotation of the BBO crystal, generating a narrowband pulse at central wavelength $600 \mathrm{~nm}$ with $\Delta \lambda_{S H}$ of $<0.41 \mathrm{~nm}\left(11.4 \mathrm{~cm}^{-1}\right)$ from a $\lambda_{F F}=1200 \mathrm{~nm}$ 
input, recorded using a fibre-coupled spectrograph (Ocean Optics HR4000). Similarly a fundamental input pulse of $\lambda_{F F}=1400 \mathrm{~nm}$ generated a narrowband pulse at $\lambda_{S H}=$ $700 \mathrm{~nm}$ with a bandwidth of $\Delta \lambda_{S H}<1.3 \mathrm{~nm}\left(26.5 \mathrm{~cm}^{-1}\right)$ measured at the limit of our low-resolution USB spectrometer (OR $1.3 \mathrm{~nm}$ ).

\subsubsection{Power conversion}

Power throughput was measured at the system input, immediately before the BBO crystal and immediately after the $\mathrm{BBO}$ crystal. The fundamental input beam was first attenuated to a pulse energy of $13.3 \mu J$ using a variable neutral density filter. In the prism configuration, the combined energy of the two pulses was measured immediately before the $\mathrm{BBO}$ to be $10.3 \mu \mathrm{J}$ at the input to the $\mathrm{BBO}$, with the narrowband signal output having an energy of $1.8 \mu \mathrm{J}$ demonstrating an $17.5 \%$ conversion efficiency for the SFG process. Comparison with other bandwidth compressors shows our conversion efficiency is acceptable, with other compressors reporting efficiencies in the range of $0.7 \%$ to $40 \%$ 104106107. SFG efficiency saturation was not achieved using the grating as the dispersive element, due to both higher power losses using a grating blazed for a Littrow geometry in a large input angle geometry, and lower power density at the $\mathrm{BBO}$ due to the wider strip length caused by higher angular dispersion.

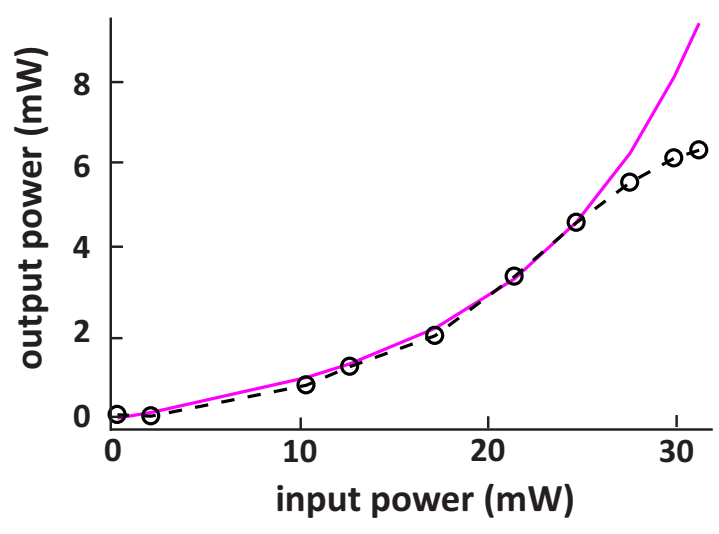

Figure 3.9: The input versus output power for the grating configuration is shown as the black dashed curve, with a Gaussian fit shown as the pink curve shows the saturation point where the two curves diverge. The optimal input power is just before saturation of the BBO crystal, where damage can start to occur. 


\subsubsection{Beam profile}

The spatial profile of the signal beam is divergent in the vertical (y) axis and collimated in the horizontal ( $\mathrm{x}$ ) axis, orthogonal to the beam propagation ( $\mathrm{z}$ ) axis. Figure 3.10 shows the measured beam profile following collimation using a $50 \mathrm{~mm}$ focal length cylindrical lens. The beam has a Gaussian intensity profile and circular symmetry with a $1 / \mathrm{e}^{2}$ width of $1.68 \mathrm{~mm} \pm 0.02 \mathrm{~mm}$.

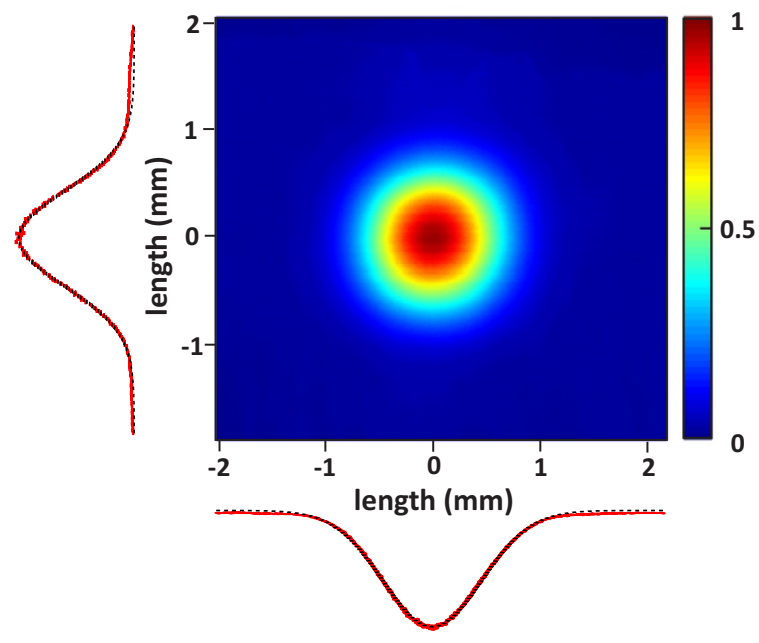

Figure 3.10: The intensity profile of the collimated signal beam, using a cylindrical lens of focal length $f=50 \mathrm{~mm}$. The red curves are intensity plots taken from the experimental data in the $x$ and $y$ axes, which each fit well to a Gaussian (black dashed curves) with a $1 / \mathrm{e}^{2}$ width of $1.68 \mathrm{~mm} \pm 0.02 \mathrm{~mm}$. This figure is reprinted with permission from reference ${ }^{92]}$ (c) The Optical Society.

\subsection{Application to stimulated Raman spectroscopy}

To demonstrate an application of our bandwidth compressor, we used the technique of FSRS in the ground state ${ }^{[568495]}$. As discussed in the previous chapter, a requirement of FSRS is an intense and spectrally narrow Raman pump laser pulse, which stimulates the emission of photons at Stokes-shifted (lower) energies. The spectral resolution of the FSRS system is determined by the spectral bandwidth of the Raman pump in the limit of the molecules vibrational dephasing time ${ }^{[84}$. We present here ground state FSRS spectra for acetone and methanol, using the spectrally narrow $400 \mathrm{~nm}$ pulse generated by our prism bandwidth compressor as the Raman pump. 


\subsubsection{Experimental method}

Figure 3.11 shows the main optical elements of the FSRS system. The generation of the Raman pump pulse is detailed in section 3.5.1 using the prism configuration. The supercontinuum (SC) pulse is generated by focusing a small potion of the fundamental laser pulse into a linearly translating $\mathrm{CaF}_{2}$ crystal to produce a probe pulse with spectral bandwidth of $\sim 350 \mathrm{~nm}$ to $800 \mathrm{~nm}$. The probe and pump pulses are horizontally (P) polarised, and focused to overlap at the center of a $1 \mathrm{~mm}$ cuvette in a non-collinear geometry.

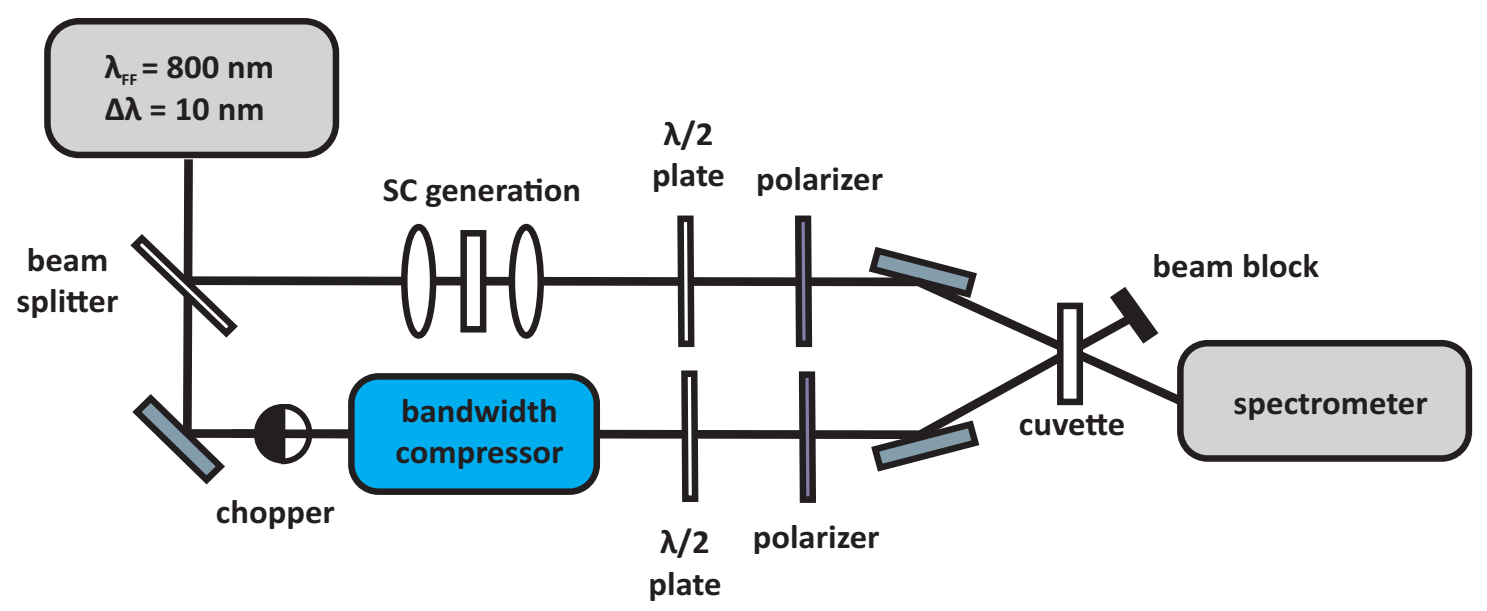

Figure 3.11: Experimental layout of the (ground state) femtosecond stimulated Raman spectroscopy system; a mode locked Ti:Sapphire amplifier delivers $3 W, 100$ $f s$ pulses at the central wavelength of $800 \mathrm{~nm}$ with a $3 k H z$ repetition rate, a beam splitter (BS) sends a small portion of the pulse for supercontinuum (SC) generation to generate the broadband probe, while the remaining power is attenuated and sent to the bandwidth compressor for generation of the narrowband Raman pump. Both pulses are overlapped at the sample, and the probe pulse is dispersed and collected on a home built Czerny Turner spectrometer. All non-marked components are plano silver mirrors.

Following interaction in the sample, the probe is sent to a home built Czerny Turner transmission grating spectrometer for data collection. The spectrometer has an OR of $20 \mathrm{~cm}^{-1}$ across a spectral range of $3100 \mathrm{~cm}^{-1}$. Spectra are collected on a CMOS detector array (Lightwise LW-ELIS-1024a-1394), at a read out rate of $3 \mathrm{kHz}$ to capture each Raman pump on-off shot. A sequence of shots with the Raman pump on and Raman pump off is generated using a mechanical chopper running at $1.5 \mathrm{kHz}$ in the Raman pump line, allowing the Raman gain spectra to be extracted and normalised by divi- 
sion of the sequential high-correlated shots of the pump-off probe spectrum.

\subsubsection{Experimental results}

Stimulated Raman spectra were obtained for acetone and methanol in a $1 \mathrm{~mm}$ cuvette with a Raman pump energy of 3.3 Data was averaged over 60,000 shots to obtain each spectrum. Figure 3.12 shows the averaged spectra normalised for each liquid showing good agreement with the expected Raman peak positions obtained from spontaneous Raman spectroscopy data (LabRAM HR800). It is of interest the relative peak intensity differences between the spontaneous and the stimulated Raman spectra observed when comparing the top and bottom panels of this figure. The intensity differences could arise due to the differing processes occurring in spontaneous and stimulated Raman scattering. Namely that the presence of self-phase modulation in the stimulated Raman process leads to the probability of stimulated emission taking place being higher than the probability for spontaneous emission to occur in particular modes, as well as inter-modal coupling [117118. For the stimulated Raman spectra presented in this figure, baseline artifacts due to cross phase modulation of the pump and probe beams were removed using a polynomial fit and subtraction computation. The FWHM bandwidth resolution of the measured spectra is $\sim 30 \mathrm{~cm}^{-1}$ demonstrating good performance of our Raman pump in comparison with other ground state FSRS systems ${ }^{[57119]}$.

\subsection{Raman pump generation using spatial filtering}

\subsubsection{Generating an IR Raman pump}

The bandwidth compressor can be tuned to create a Raman pump at $800 \mathrm{~nm}$ by changing the incoming fundamental beam wavelength to $1600 \mathrm{~nm}$, however this proves challenging in terms of available power. The TOPAS used to generate the incoming beam into the bandwidth compressor (that is to be doubled) produces power in the range of $m W$ in the IR, specifically at $1600 \mathrm{~nm}$ the output power is $\sim 100 \mathrm{~mW}$. In order to generate a compressed $800 \mathrm{~nm}$ beam by doubling the $1600 \mathrm{~nm}$ beam through the bandwidth compressor, the conversion efficiency is too low to generate an intense enough Raman pump at $800 \mathrm{~nm}$. It was decided then to revert to the classic, albeit "lossy", technique of spatial compression using a grating and a slit in a four-f configuration ${ }^{[57}$. Chapter 4 discusses in more detail the two spectrometers, in terms of their build, operation and application to this project. 

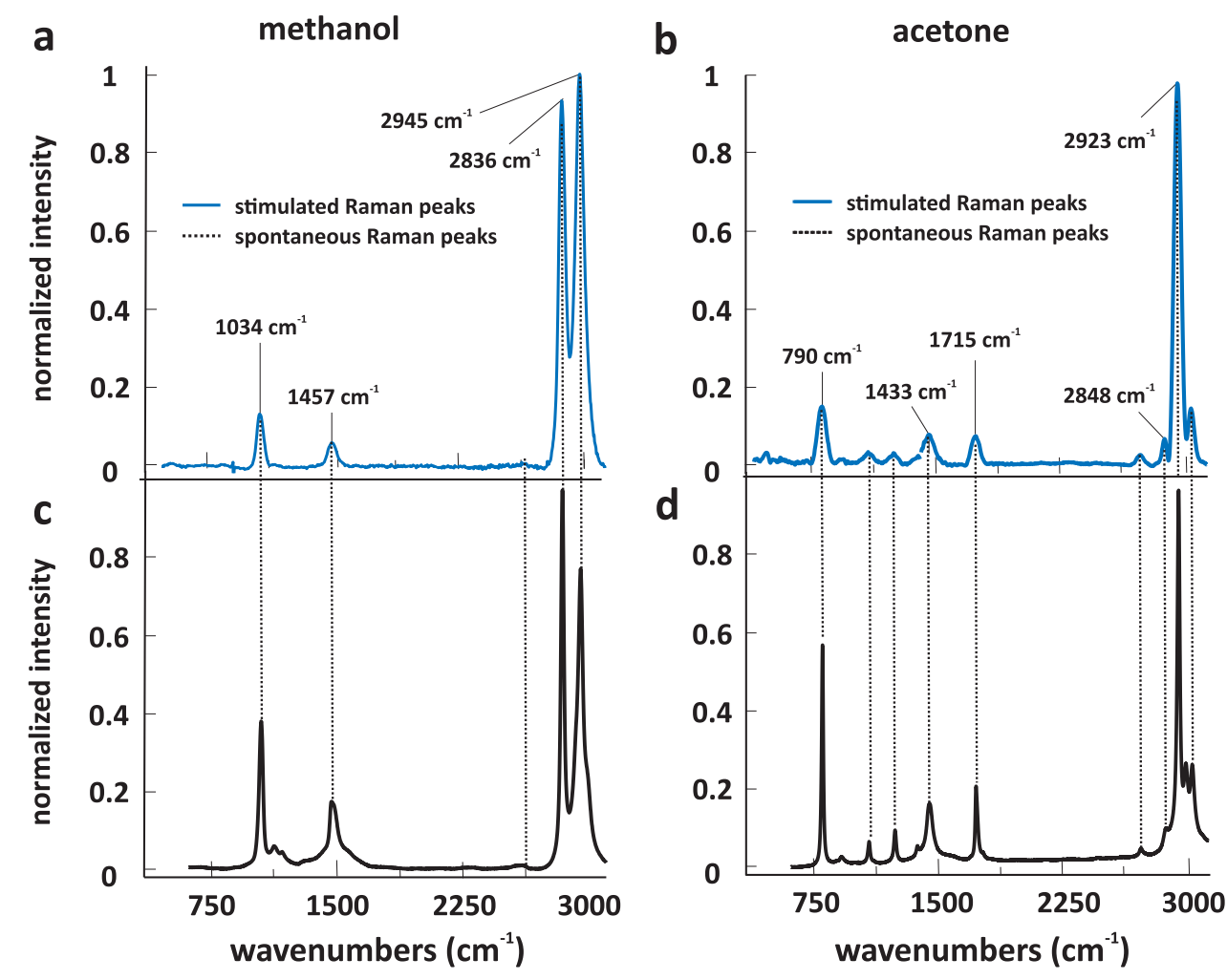

Figure 3.12: Normalised stimulated Raman spectra (blue curves) for (a) methanol and (b) acetone liquids generated using FSRS with the Raman pump generated by the frequency domain bandwidth compressor. Panels (c) and (d) show the normalised spontaneous Raman spectra for the same liquids generated using a spontaneous Raman spectroscopy system (LabRAM HR800) with the peak matches between the two experiments indicated by the black dashed lines. This figure is adapted with permission from reference ${ }^{[2]}$ (C) The Optical Society.

\subsubsection{Generating an IR Raman pump using a spatial filter}

A narrowband Raman pump at a central wavelength of $800 \mathrm{~nm}$ can be generated from the broadband fundamental using a spatial filter. In this configuration the fundamental beam is sent to a grating which disperses the beam into its colours. A lens of power $f$ placed at distance $f$ from the grating collimates the beam and a slit placed at the Fourier plane selects a colour, whose bandwidth is proportional to the slit width. The slit can also be translated across the beam to fine tune the wavelength. In the folded configuration shown in figure 3.13, a planar mirror is placed as close as possible behind 


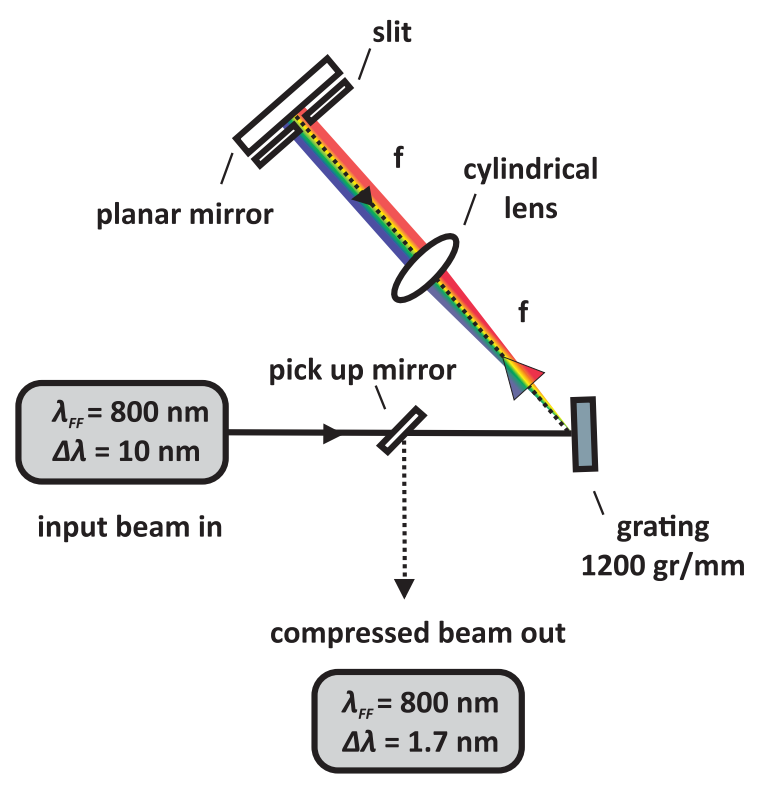

Figure 3.13: Generating a narrowband IR Raman pump using a spatial filter in a folded $4 f$ configuration. Here, the fundamental laser pulse is dispersed using a $1200 \mathrm{gr} / \mathrm{mm}$ grating and focused to a slit in the Fourier plane which selects the colour. A planar mirror reflects the narrowband beam back through the system to undo the dispersion, at a slightly lower geometry, and a pick up mirror sends the compressed pulse to the sample.

the slit to send the narrowband beam back through the system in order to undo the spatial chirp and compensate for the group velocity dispersion (GVD) caused by the grating. The returning beam is displaced vertically so that, after passing back through the lens and the grating, it can be picked off using a D-shaped pick up mirror and sent to the sample.

While this bandwidth compression system is relatively straight forward to implement, it has notoriously low energy throughput $(\sim 0.02 \%)$, as the narrowband beam is produced by filtering out most of the fundamental pulse with a slit. Because of this, a large proportion of the fundamental beam $(0.7 \mathrm{~W})$ is used to generate a usable narrowband Raman pump of sufficient power. The system setup used a reflective grating with a $1200 \mathrm{gr} / \mathrm{mm}$ groove density, and a cylindrical lens of power $f=150 \mathrm{~mm}$. In this configuration, a $20 \mu \mathrm{m}$ slit was used to produce an $800 \mathrm{~nm}$ Raman pump with a bandwidth of $39 \mathrm{~cm}^{-1}$, with $7.7 \mathrm{~mW}$ power, and a fluence at the sample of $6601 \mu \mathrm{J} / \mathrm{cm}^{-2}$. Panel $a$ of figure 3.14 shows the bandwidth compression of the fundamental pulse for 3 slit 

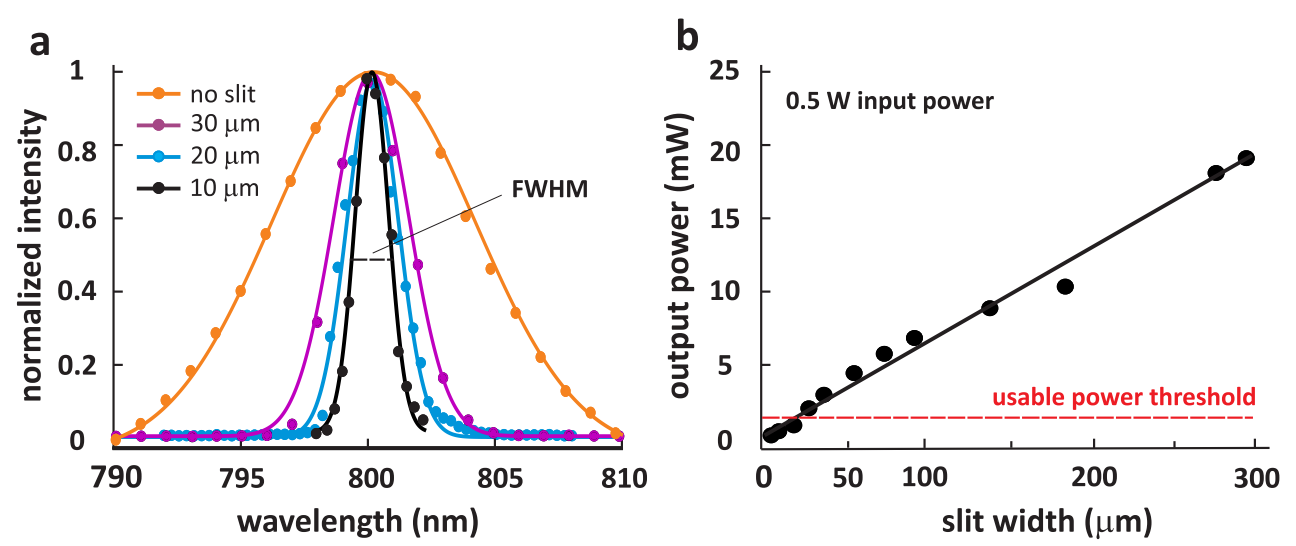

Figure 3.14: Panel (a) shows the normalised spectral compression of the fundamental pulse through the spatial filter for 3 slit widths; panel(b) shows the power conversion of the fundamental pulse as a function of slit width, with the red dashed line indicating the power threshold level required to generate a Raman pump of sufficient intensity to resolve Raman peaks.

widths. A FWHM of $1.44 \mathrm{~nm}\left(22.5 \mathrm{~cm}^{-1}\right)$ is achieved for a slit width of $10 \mu m$, however the energy throughput at this bandwidth is not sufficient to generate a Raman pump of sufficient intensity. A slit width of $20 \mu \mathrm{m}$ achieves a bandwidth of $2.5 \mathrm{~nm}\left(39.0 \mathrm{~cm}^{-1}\right)$ achieves $\sim 1 \mathrm{~mW}$ of power, and is sufficient to resolve Raman peaks in the limit of the spectrometer's resolution. Panel $b$ shows the power conversion for an input power of $0.5 \mathrm{~W}$, with the red dashed line indicating the threshold power level required to generate a usable Raman pump.

\subsection{Chapter summary}

In this chapter two methods for producing a Raman pump in the visible $(400 \mathrm{~nm})$ and IR $(800 \mathrm{~nm})$ are presented. The first is a novel, experimentally straightforward method for generating intense narrowband picosecond pulses by nonlinear second harmonic conversion in the frequency domain of femtosecond pulses in the presence of equal and opposite spatial chirp. Our nonlinear bandwidth technique successfully addresses tradeoffs between achievable bandwidth compression, power conversion efficiency, frequency tunability and experimental simplicity in current bandwidth compression methods. The system generates frequency tunable picosecond pulses with a spectral bandwidth of $20.0 \mathrm{~cm}^{-1}$, power conversion efficiency of $\sim 18 \%$ and Gaussian tempo- 
ral profile, operable across a broad spectral range. One application of our bandwidth compressor, FSRS in the ground state, has been demonstrated on liquids. We anticipate that the fine and broad tunability of our system, and its simplistic and portable design will be highly appealing for use in spectroscopic applications where both high spectral and temporal resolution are required, such as time-resolved vibrational spectroscopy, and single photon compression for quantum information transfer applications.

Potential adaptations of our bandwidth compression system to improve efficiency include the use of a transmissive dispersive element, such as a transmission grating. Further potential adaptations include using a non-collinear geometry in the vertical plane, rather than the horizontal plane, to allow collinear mixing of the two chirped strips and remove potential compression bandwidth limitations due to geometrical smearing caused by large non-collinear incident angles to the BBO.

The second method presented for Raman pump generation is the classical linear method of spatially filtering the fundamental $800 \mathrm{~nm}$ pulse, and is used to generate an $800 \mathrm{~nm}$ Raman pump with sufficient intensity to resolve both ground state and dynamic Raman peaks for materials.

In the following chapters, both methods are used in the FSRS experiments conducted on eumelanin and its DHI and DHICA monomers. The method chosen for each experiment is dependent on the PIA band of the molecule of interest, where the $400 \mathrm{~nm}$ Raman pump achieves resonance with the FSRS system build test case, napthalene, as described in section 4.6 of chapter 4.1. as well as the DHICA monomer discussed in section 5.4 .3 of chapter 5 . The IR Raman pump is used in experiments conducted on eumelanin in chapter 6, whose PIA band is at $1.3 \mathrm{eV}(953 \mathrm{~nm})$. Future work may include adjustments to the TOPAS system which generates input pulses to the bandwidth compressor in the IR, so that sufficient power is achieved in the Raman signal pulse at longer wavelengths. This would render the spatial filtering method redundant, and the bandwidth compressor could then be used for FSRS experiments spanning the visible to IR regions. 


\section{Chapter 4}

\section{Femtosecond stimulated Raman spectroscopy - system build}

The FSRS method introduced in chapter 2 is described in detail in this chapter. This includes details about the optical build set-up, system testing and improvements, data collection, data processing and post-processing analysis methods used in the FSRS experiments.

\subsection{Experimental configuration}

To implement FSRS as an experimental technique, the transient absorption (TA) spectroscopy system developed by Hodgkiss and coworkers ${ }^{40 \mid}$ has been extended with the addition of a narrowband Raman pump pulse discussed in chapter 3, a high resolution spectrograph to record Raman gain spectra, and the addition of new post-processing software scripts.

Figure 4.1 shows a schematic representation of the main components in the FSRS system. Full technical details of the optical layout is available in appendix A. Our $3 W$, $3 \mathrm{kHz}$ Ti:Sapphire amplifier produces laser pulses at a central wavelength of $800 \mathrm{~nm}$ with a temporal bandwidth of $\Delta \tau=100 \mathrm{fs}$ (FWHM) and spectral bandwidth of $\Delta \lambda=$ $11 \mathrm{~nm}$ (FWHM).

To generate the excitation pump from the fundamental laser source, a second and third harmonic generator were built as described in section 4.2. The excitation pump is first optically steered down an electronically controlled delay stage (Newport $M-$ 


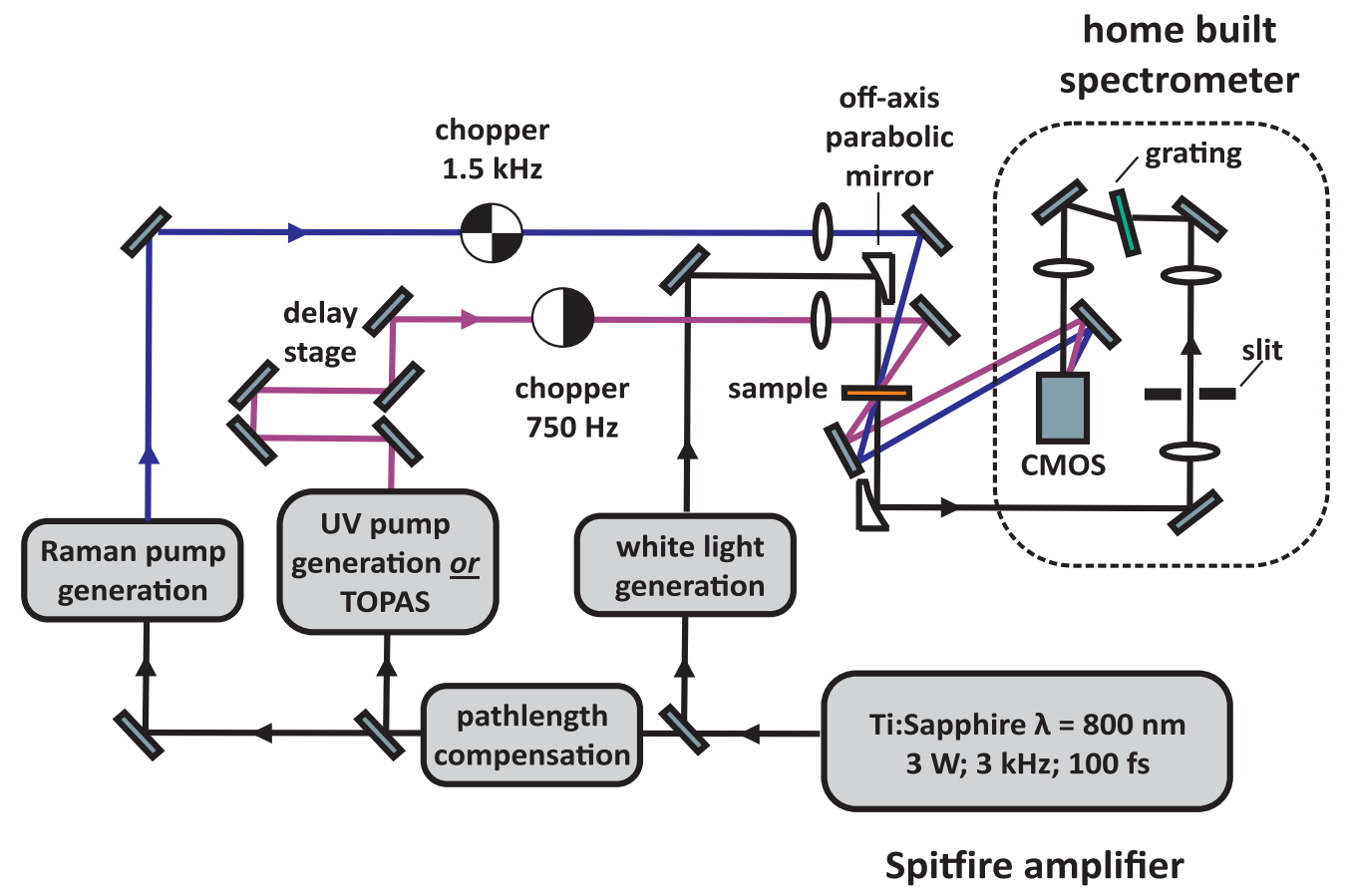

Figure 4.1: The key elements of the FSRS system build are shown in this schematic, with the Raman pump, excitation pump and white light probe being generated from the Ti:Sapphire fundamental laser source. Pathlength compensation is required to ensure all the pulses arrive together in time at "time zero" before the delay stage then allows time delay steps to be resolved. The three pulses shown in black, blue and purple, are overlapped in space at the sample position, with the probe pulse then sent to the home built spectrometer. Reference pulses from the Raman pump and excitation pump are sent to the CMOS camera so that shot-toshot references analysis can reveal, post processing, the ground state Raman spectra (GS), the dynamic Raman spectra in the excited state (ES) (excitation pump "on"), as well as the transient absorption spectra (TA). The FSRS signal is then calculated from these shots.

$1 M S 500 C C H A)$ in order to capture Raman spectra in $f_{s}$ time steps across a $n s$ time region between photo-excitation and the arrival of the Raman pump-probe pair.

The supercontinuum probe is generated from the fundamental $800 \mathrm{~nm}$ laser pulse as 
described in section 4.2.3. The Raman pump is generated using either a frequency bandwidth compressor, or a spatial filter, as described in section 3.8 .2 of chapter 3 . The two pump pulses are pathlength matched in order to temporally overlap with the probe, and all pulses are then spatially overlapped and focused to the sample in a noncollinear geometry. The probe beam, containing the heterodyned Raman gain signal, is dispersed and collected using a home-built high resolution transmission grating spectrograph.

Two choppers set at $750 \mathrm{kHz}$ in the excitation pump arm and $1.5 \mathrm{kHz}$ in the Raman pump arm create a sequence of 4 pump "on" - pump "off" shots, described in section 4.4.3, allowing the calculation of the Raman gain from sequential shots in both ground and excited states, as well as the transient absorption spectra across a particular spectral region.

\subsection{Pulse generation}

There are three pulses required to perform FSRS which are the Raman pump, the excitation pump and the white light probe. The generation of these three pulses from the fundamental $800 \mathrm{~nm}$ laser pulse are described in the below sections.

\subsubsection{Raman pump}

The two different Raman pump wavelengths used in these experiments, $400 \mathrm{~nm}$ and $800 \mathrm{~nm}$, are generated using two different bandwidth compression methods. These methods are described in detail in chapter 3

\subsubsection{Excitation pump}

The measurements discussed in this thesis are performed using two different excitation pump excitation wavelengths in the UV $(267 \mathrm{~nm})$ and the visible regions $(400 \mathrm{~nm}$ ). Home-built second and third harmonic generators were built to generate these pump wavelengths from the $800 \mathrm{~nm}$ fundamental laser pulse. 


\section{Third harmonic generation}

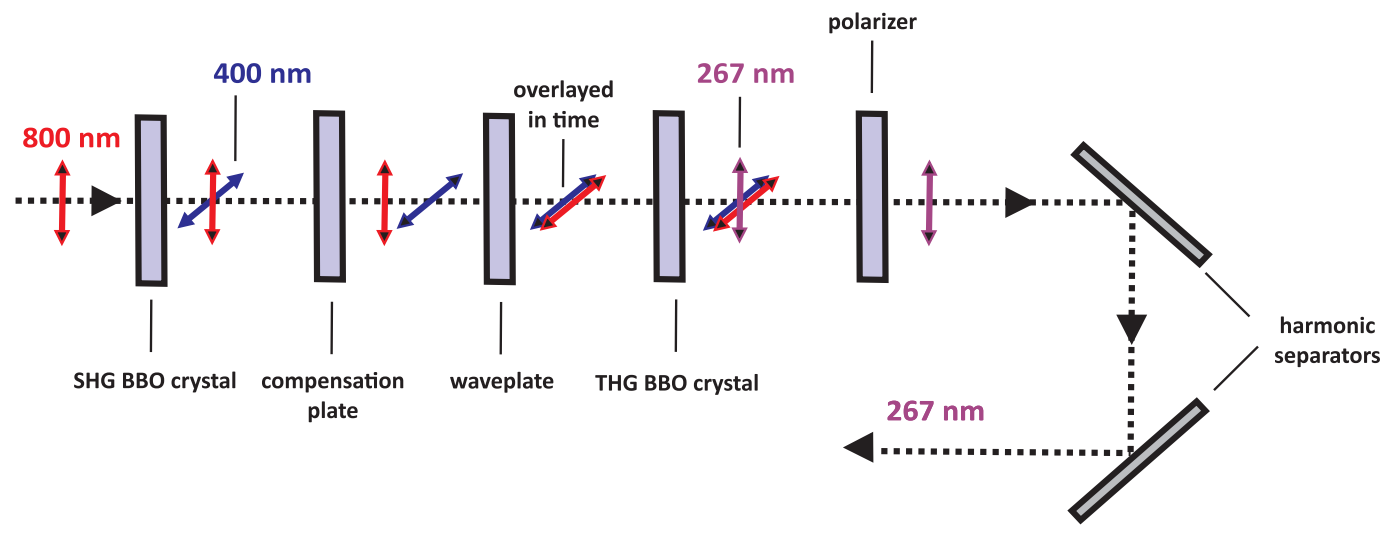

Figure 4.2: Second and third harmonic generation using a straight forward optical system. The first optical element converts a portion of the fundamental into its second harmonic; the next component compensates for the group velocity dispersion between the transmitted fundamental and its second harmonic by the following waveplate, which is used to align their polarisations. Then, a third harmonic BBO crsytal generates UV light at $267 \mathrm{~nm}$. A polariser and two harmonic separators then suppress the residual fundamental and second harmonic wavelengths, with the harmonic separators also working as steering mirrors.

Third harmonic generation (THG) involves the conversion of the fundamental $800 \mathrm{~nm}$ pulse into its third harmonic using a combination of optical elements in the collimated configuration as shown as figure $4.2^{64120]}$. The "S" polarised $800 \mathrm{~nm}$ fundamental pulse first propagates through a type 1 second harmonic generation (SHG) BBO crystal, converting a portion of the beam into a " $\mathrm{P}$ " polarised second harmonic pulse at $400 \mathrm{~nm}$. A calcite plate then compensates for the group velocity delay (GVD) of the fundamental and second harmonic pulses caused by the upcoming waveplate. The third optical element is a zero order waveplate, anti-reflective (AR) coated for $800 \mathrm{~nm}$ and $400 \mathrm{~nm}$, which polarises the $800 \mathrm{~nm}$ fundamental beam to "P" polarisation. The fourth stage is a type 1 THG BBO crystal, which generates an "S" polarised UV pulse at $267 \mathrm{~nm}$. The pulse then propagates through a wire-grid polariser which selects " $\mathrm{S}$ " polarised light, allowing the third harmonic to pass through, while blocking the residual fundamental and second harmonic wavelengths. The fundamental input beam diameter was first reduced from $\sim 12 \mathrm{~mm}$ to $\sim 4 \mathrm{~mm}$ (FWHM) using a home-built $3: 1$ telescope in order to physically fit the beam through the optics apertures of the 
system, as well as increasing the beam fluence, and thus conversion efficiency. Two harmonic separators placed after the polariser are used as fine adjustment steering mirrors, and further suppress the fundamental and SHG wavelengths by reflecting the third harmonic and transmitting the second harmonic. Experimentally, efficient power conversion of the fundamental $800 \mathrm{~nm}$ pulse into the third harmonic proved to be challenging. This was assigned to poor beam mode quality, a large beam spot size and perhaps temporal chirp of the fundamental pulse. The telescope used to reduced the beam size greatly improves the throughput, but still only about $0.5 \%$ conversion was achieved, where calculations would expect an $\sim 8 \%$ conversion efficiency for the same configuration ${ }^{[120 \mid}$. However, a pulse energy of $0.3 \mu \mathrm{J}$ was achieved at the sample, which is more than sufficient to stimulate Raman photon emission.

Experimentally, it is straight forward to convert between generating the second and third harmonic, as the first stage of the third harmonic generation is the second harmonic generation. By removal of the subsequent optics following second harmonic generation (aside from the polarisor) an intense pulse at $400 \mathrm{~nm}$ can be generated with high conversion efficiencies of $\sim 25 \%$. The SHG and THG setup was built in conjunction with co-worker Aleksandra Ilina, of the Victoria University of Wellington.

\subsubsection{The probe: white light generation}

White light generation (WLG) is a process where the fundamental $800 \mathrm{~nm}$ laser pulse with a bandwidth of $\sim 11 \mathrm{~nm}$ is converted into a broadband supercontinuum spanning the UV, visible and infrared (IR) regions $\frac{6265+68]}{6}$. When an ultrashort laser pulse of sufficient intensity is focused into a suitable nonlinear medium such as a yttrium aluminium garnet (YAG) or calcium fluoride $\left(\mathrm{CaF}_{2}\right)$ crystal, a series of nonlinear processes generates a new supercontinuum laser pulse. One of the key nonlinear processes is self-phase modulation, generating the optical Kerr effect, in which the intensity of the laser pulse changes the refractive index of the material, causing the beam to self focus and create filaments generating a new pulse with a broad spectral range. Together key nonlinear processes to the generation of a supercontinuum include self-steeping, four-wave mixing, and ionisation, which are discussed in detail elsewhere $6265 \mid[68$.

Two different medium are used for each Raman pump configuration. For the visible region a $\mathrm{CaF}_{2}$ crystal is used to generate a supercontinuum spanning the $400-450 \mathrm{~nm}$ region of interest. The $\mathrm{CaF}_{2}$ crystal is continuously translated using a home-built stage 
in order to avoid crystal damage ${ }^{[6]}$. For the second configuration a YAG crystal is used to generate an IR probe spanning the $800-1100 \mathrm{~nm}$ region of interest. Rastering of the YAG crystal is not deemed necessary due to its high damage threshold.

Generating stable white light is essential for good SNR in resolving the ground state Raman (GS), excited state Raman (ES) and transient absorption (TA) signals. The stability of the probe is monitored during the experiment by observing the normalised shotto-shot difference of the probe pulse (in the absence of either pumps), which should be on the order of $\mu-m$ counts on the detector for a typical probe intensity of about 30,000 counts. Factors contributing to the stability of the white light include the fundamental beam mode quality, the input power and the alignment of the fundamental beam into the nonlinear crystal. To maximise the mode quality of the beam, a spatial filter is used, involving two irises placed at a distance apart before propagation through the crystal. The first iris selects the centre of the beam to be Gaussian in profile (TEM $\mathrm{T}_{00}$ mode), and the second iris clips the diffraction pattern caused by the first iris. The further apart the irises are the better the beam mode quality (due to the increasing size of the diffraction pattern upon distance from the first iris). To avoid crystal damage and multiple filamentation, the input pulse is set at the minimum power possible using a variable density filter, typically at a $1 \mu \mathrm{J}$ pulse energy. Focusing the beam first to the front of the crystal, and then translating slowly towards the centre while visually monitoring the supercontinuum beam allows the optimal position for stability for the lowest input power to be found. Finally, the beam path through the crystal is aligned to be as close to perpendicular to the crystal surface as possible using a webcam, detailed in appendix A. Following white light generation, the probe is collimated with an off axis parabolic (OAP) mirror, steered and focused to the sample as shown in figure 4.1 .

\subsection{Beam improvements}

\subsubsection{Spot size}

An important element in FSRS is to ensure not only that the three pulses are aligned in space at the sample, but also that the beam profiles are as close to $\mathrm{TEM}_{00}$ as possible. The excitation pump beam should be the largest in diameter, followed by the Raman pump, with the probe being contained within each. This is so the entire probe is modulated, maximising the gain signal, and that any spatial chirp artefacts in the probe are reduced. Figure 4.3 shows the configuration used in this set up as well as fluences for 


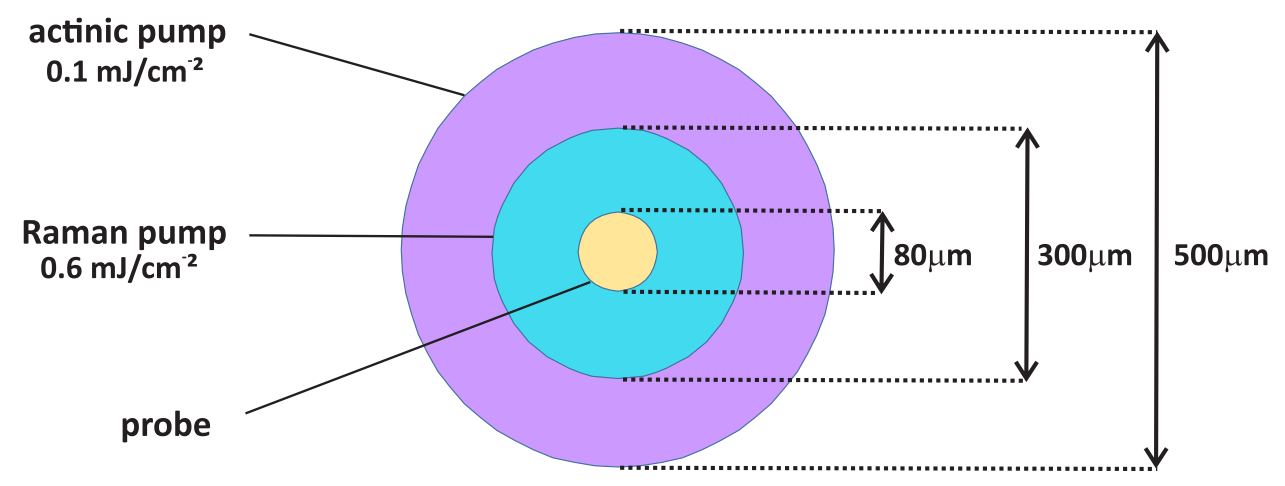

Figure 4.3: The spot size and fluence for each of the three laser beams at the sample position, with the excitation pump shown in purple; the Raman pump shown in blue, and the probe shown in yellow.

each pump.

\subsubsection{Beam profile}

During the build and alignment process, astigmatism of the white light probe, caused primarily by the off-axis parabolic (OAP) alignments, is closely monitored using a webcam. By carefully adjusting the $x-y-z$ positions of the OAPs, the spot size and profile of the probe beam is vastly improved as shown in figure 4.4 . All three beams are spatially aligned using a webcam. The temporal alignment of the three beams is conducted using a methylammonium lead iodide $\left(\mathrm{MAPbI}_{3}\right)$ perovskite film with a strong and long lived ground state bleach signal (on the order of $n s$ ). For each pump-probe pair, "timezero" (that is, the initial temporal overlap) is found by observing the rise of the TA signal for both excitation pump/probe and Raman pump/probe configurations. The material to be measured is then positioned in the sample holder and translated along the probe beam propagation axis until the TA rise signal is visible in real time, and then optimised. The temporal Raman pump / probe overlap is then fine tuned by adjusting the temporal overlap of the Raman pump using a manual delay stage until the Raman peaks are optimised. The crossing angle of the three beams is minimised to be approximately $10 \%$ between each beam to ensure maximum temporal interaction at the sample, as the beams exist together for longer in an ideally co-linear geometry. 


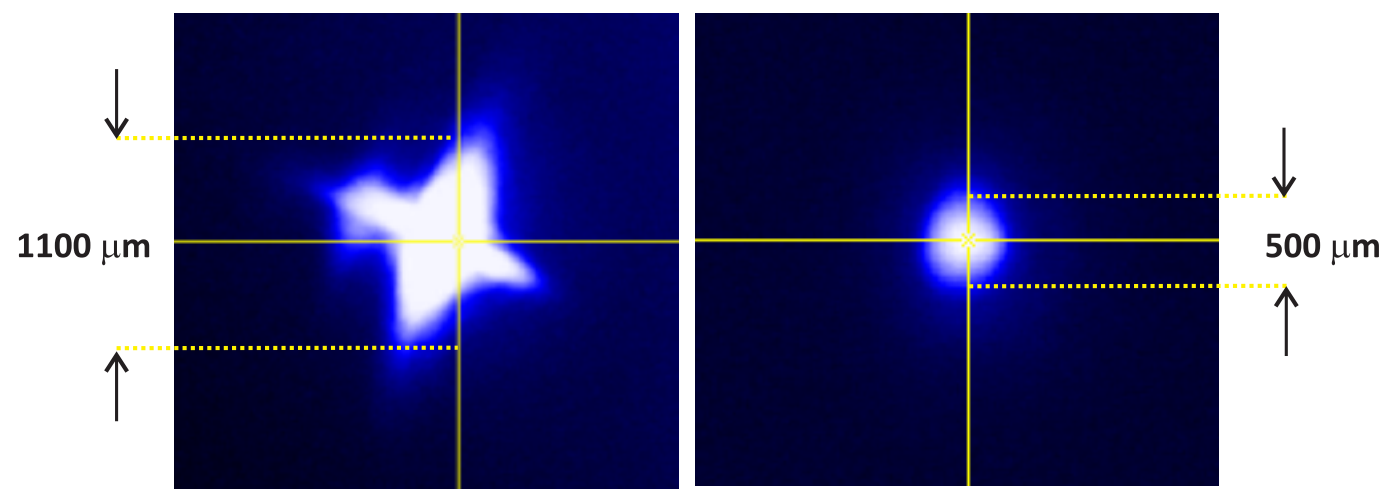

Figure 4.4: Astigmatism of the white light probe beam at the sample is recorded on a wecbcam in the left hand panel; while the right hand panel shows a smaller spotsize with an improved beam profile by careful alignment of the off-axis parabolic mirrors during the experimental set up. The probe spot-size used in these experiments was $300 \mu m$.

\subsection{Data collection}

\subsubsection{High resolution spectroscopy}

To resolve Raman gain spectra across the vibrational energy manifold, a high-resolution spectrometer is needed to collect and record Raman signals with both high digital and spectral resolution. Spectral bandwidth resolution of order $20 \mathrm{~cm}^{-1}$ (or $0.2 \mathrm{~nm}$ at the central wavelength of $400 \mathrm{~nm}$ ) is required to resolve Raman gain peak bandwidths. This can be justified as the Raman peaks of eumelanin are in themselves broadband, with two main peaks of $\sim 150 \mathrm{~cm}^{-1}$ bandwidth spanning over a $\sim 500 \mathrm{~cm}^{-1}$ spectral range, as shown in figure 6.6 in chapter 6. A spectral range of $3000 \mathrm{~cm}^{-1}$ (corresponding to a spectral range of $\sim 50 \mathrm{~nm}$ for a Raman pump at wavelength $400 \mathrm{~nm}$ ) is required to capture the entire Stokes shifted spectra.

A Czerny-turner transmission grating spectrometer is chosen as the spectrometer design [121122]. The layout of the spectrometer is shown in the upper right panel of figure 4.1. The Czerny-turner spectrometer uses a slit at the entrance whose width is proportional to the resolution of the system - also meaning a key factor in the design is the consideration of energy throughput. A transmissive grating of groove density 600 $\mathrm{gr} / \mathrm{mm}$ (Thorlabs GTU25-06) is used based on its $\sim 80 \%$ throughput in the first order at $400 \mathrm{~nm}$, and 2 inch concave aluminium mirrors (Eksma 095-0115R-200) are used as 
the focusing elements.

The spectral resolution of a spectrometer is limited by either the optical or digital resolution of the system. The optical resolution is determined by the input slit width, the focal length of the optical system, and the grating dispersion. The narrower the slit width, the higher the optical resolution. The digital resolution is determined by the pixel width of the detector and the dispersion of the grating. In order to determine the correct optical components to achieve sufficient spectral resolution (and sufficient energy throughput) to resolve the Raman peaks, calculations are required beginning with the well known grating equation [123]124

$$
\sin \alpha+\sin \beta=k n \lambda,
$$

which determines the dispersion of the input pulse, $\Delta \delta$, using the input angle of the beam to the grating, $\alpha$, the output angle for each wavelength, $\beta$, for each the specific wavelength $\lambda$, and the groove density of the grating $k$ (with $n$ being the refractive index of air, 1).

Assuming 1:1 magnification, a resolvable feature at the detector is the same physical size as the slit width. Three pixels are required to resolve a feature at the detector (assuming that feature is a Gaussian) so we can use this to determine the dispersion variables from the grating dispersion, and therefore the focal lengths and slit width required using the camera detector length and pixel width information. It should be noted that an addition limit to the spectrometer is the actual bandwidth of the Raman pump (in this case about $20 \mathrm{~cm}^{-1}$ ), meaning that with, for example, infinitely high optical and digital resolution in the spectrometer, the Raman pump bandwidth then sets the resolution limit of the system, in terms of resolving Raman peaks.

Two spectrometers were built during this project as discussed in chapter 3 . The same resolution calculator is used for each spectrometer, however two different optical systems were required due to the different spectral ranges of interest and the different cameras used. Specifically, the digital specifications of the CMOS camera (Lightwise LW-ELIS-1024a-1394) are: $d=8 \mathrm{~mm}$, pixel size $=8 \times 8 \mu \mathrm{m}$, with 1024 pixels. The digital specifications of the Stresing photo diode array (Stresing dual line PDA with Hamamatsu 256 pixels sensors) are: $d=12.8 \mathrm{~mm}$, pixel size $=50 \times 2500 \mu \mathrm{m}$, with 256 pixels. As the same calculations were used to determine the appropriate optical elements for 


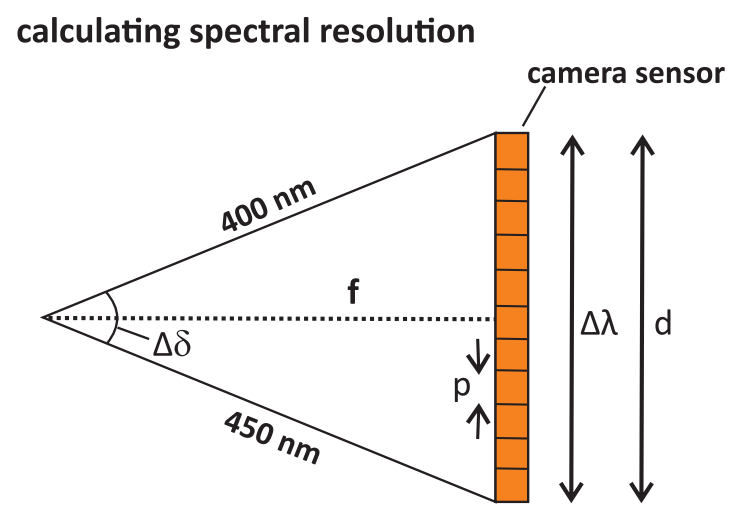

Figure 4.5: Once the grating dispersion $\Delta \delta$ is calculated for each wavelength, the appropriate optical elements can be selected to ensure the optical and digital resolution of the spectrometer is higher than the Raman pump bandwidth. The length of the camera detector, $d$, and pixel width, $p$, allows suitable focusing elements of power $f$ to be chosen. Assuming a 1:1 magnification of the system, a feature at the detector is the same width as the slit width (in this case, $20 \mu \mathrm{m}$ ), and must be at least 3 pixels in width in order to be resolved as a feature.

sufficient resolution, just one spectrometer (used for the $400 \mathrm{~nm}$ Raman pump) will be discussed here.

Figure 4.5 shows how the dispersion of wavelengths $\Delta \delta$ determined by the grating equation 4.1. can be then mapped onto the camera detector of length, $d$, in order to ensure the the full spectral range of interest, $\Delta \lambda$, fills the detector while a resolvable feature (determined by the slit width) is at least three pixel widths ( $p$ ) wide.

In both spectrometer designs, different optical components are used in order to ensure the spectral resolution is less than the Raman pump bandwidth. Further information for each design can be found in appendix A

During the build process, the spectrometer setup is improved using a blue continuous wave (CW) laser (Thorlabs LD55) and a shearing interferometer (Thorlabs SI254) in order to precisely determine the distances the optics should be placed apart for maximum resolution at the detector, and to minimise coma and astigmatism. The resolution of a spectrometer would normally be tested using a lamp, however this was unsuccessful as the low throughput of the spectrometer (due to the narrow slit width) didn't allow enough counts to be collected at the detector. However, the Raman pump peaks 
tested for the calibration standard described in section (4.5.1) were measured at the Raman pump bandwidth limit, thus the spectral resolution of the spectrometer (without being able to precisely measure it) is deemed to be higher than the Raman pump peak resolution, which is the ideal scenario.

\subsubsection{Shot collection}

Raman gain intensities are calculated from a series of four sequential "pump-on" and "pump-off" shots, generated by two mechanical choppers in the excitation pump beam path, and the Raman pump beam path. For the Raman pump, a chopper running at $1.5 \mathrm{KHz}$ blocks every second laser pule. For the excitation pump, a chopper running at $750 \mathrm{~Hz}$ blocks the first two pulses and allows the next two through. The choppers generate the shot sequence shown in figure 4.6, defined as shots $A, B, C$, and $D$.

For each time point 75, 000 shots are captured for each individual chopper sequence and processed in a data stack as intensity $I$ as a function of wavelength $\lambda$ and time $\Delta \tau$. This is carried out in 10 delay sequencing runs, capturing 300, 000 shots for each time point for 10 runs (a total of 300,000 shots per time point, divided by 4 for each shot sequence giving a total of 75, 000 shots per sequence). Averaging across the 75,000 sequence shot stack increases SNR ratios and reduces the impact of any outlying data due to fluctuations in the white light probe pulse. In initial experiments, 6000 total shots per time point were captured over a very dense time range of 260 time points over a $n s$ time region (as per usual TA experimental conditions). However, it became apparent that Raman features (apart from in highly active Raman solvents) can't be resolved using a low shot averaging regime, hence the shot numbers are vastly increased, and the time point density decreased (to 28 time points) to maintain good SNR of the Raman gain features, whilst keeping collection times reasonable.

The Raman gain signal is extracted post processing from the averaged pulse sequence and processed as shots $(A-B) /(C-D)$ and Raman spectra plotted as a function of time delay from photo-excitation, $\Delta \tau$, as shown in figure 4.6. It is important that the two choppers are phase locked together to avoid phase drift between them, as otherwise the laser pulses can "bleed" between each chopper blade and and not give a true "on" or "off" shot. This is achieved mechanically by mode locking the two controller boxes of the two choppers together - chopper "one" is triggered by the laser, and chopper "two" is triggered by chopper "one". The phase of each chopper can be electronically 


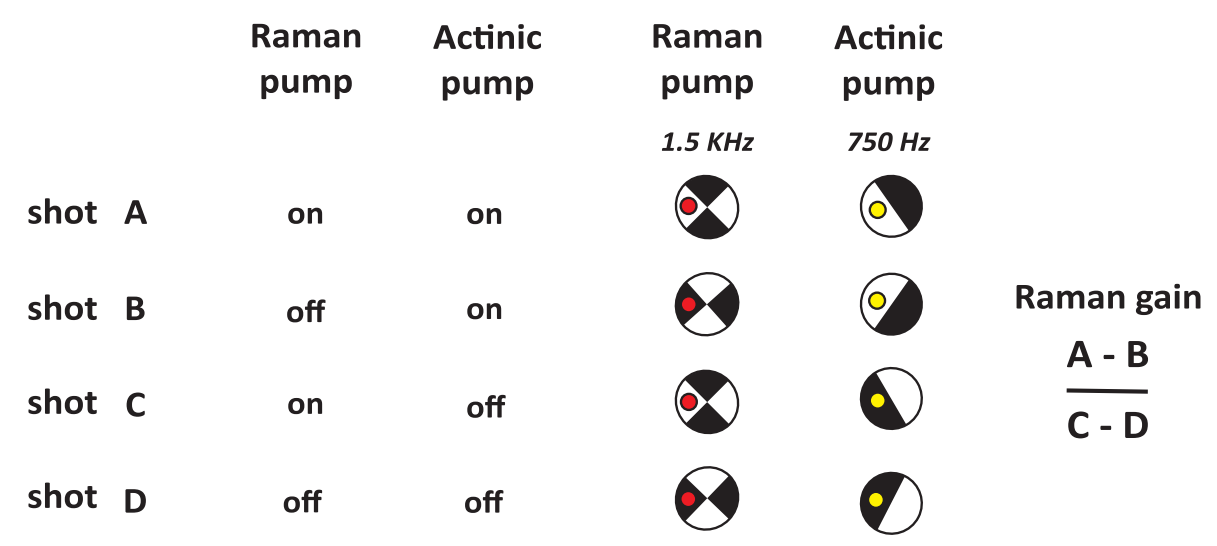

Figure 4.6: Two mechanical choppers are used to obtain the four shot sequence from the excitation and Raman pumps. The fundamental beam has a repetition rate of $3 \mathrm{kHz}$. Chopper "one" chops the Raman pump at a frequency of $1.5 \mathrm{kHz}$ (i.e on-off-on-off). Chopper "two" chops the excitation pump at a frequency of $750 \mathrm{~Hz}$ (on-on-off-off), creating a continuous 4 shot sequence, shot $A, B, C$ and $D$ throughout the data stack

adjusted while visually observing the beam after passing through the chopper, as the blade edges are seen to visibly translate across the beam if the chopper is not in phase with the laser pulses. It is also worth noting that the shot to shot correlation is important for the Raman pump in order to maximise SNR in the gain signal, hence the Raman pump is chopped at $1.5 \mathrm{kHz}$ so that subsequent Raman "on" and "off" shots are at maximum correlation. This is because shots $A$ and shot $B$ collected for the probe are more correlated than shots $A$ and shots $C$, due to intensity fluctuations of the probe pulses.

\subsubsection{Chopper referencing}

In order to assign shots post processing, shot referencing of the two choppers is required. As already discussed, there are two spectrometer configurations used involving two different camera types: Camera "one" is a CMOS detector (used in the spectrometer for the $400 \mathrm{~nm}$ Raman pump configuration over a $400-450 \mathrm{~nm}$ range) and camera "two" is a PDA detector (used in the spectrometer in the $800 \mathrm{~nm}$ Raman pump 


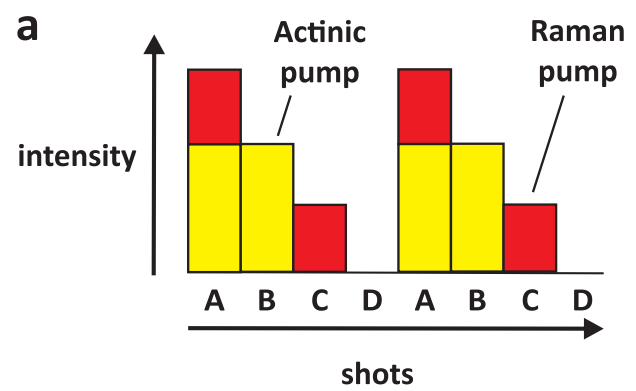

b
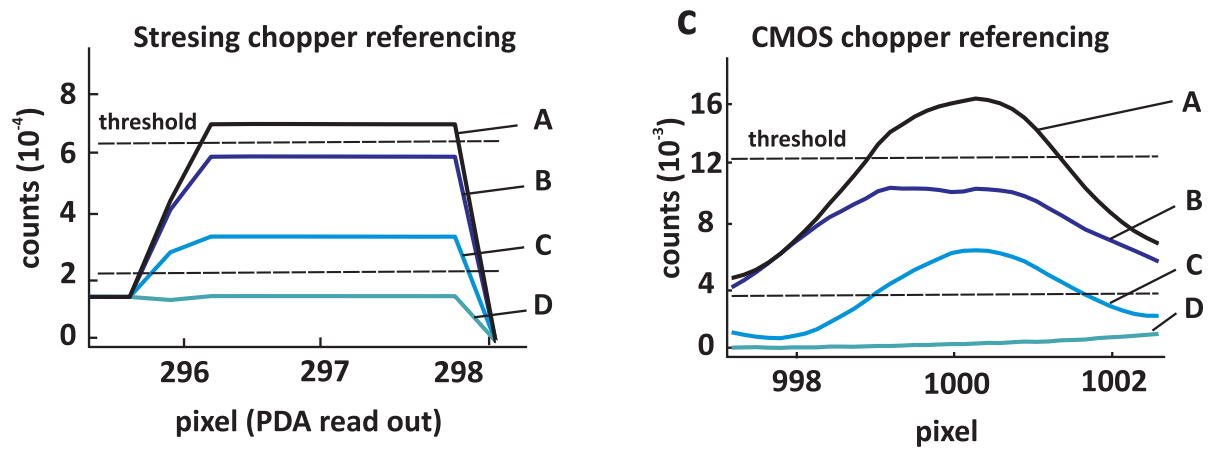

Figure 4.7: Post processing shot sequencing is achieved by calculating the intensity of the excitation and Raman pumps at the detector (either via pixel reference or PDA reference). Here, in panel $a$, the first shot - shot $A$ - contains both the excitation (yellow) and Raman (red) pump intensities being "on", however the next shot "B" has only the excitation pump "on". The third shot " $\mathrm{C}$ " has only the Raman pump "on", and the last, they are both "off". From this intensity sequence, using a "high" and "low" threshold value, it can be determined which shot is which. An example shown of a four shot data sequence captured for PDA referencing is shown in panel " $b$ " and, the same example for the CMOS referencing via pixel is shown in panel "c"

configuration over a $800-1100 \mathrm{~nm}$ range). For both detectors, shot referencing is achieved using two continuous wave lasers pointed through each respective chopper at the pump location to capture "on" (the laser pulse passes through) versus "off" (the laser pulse is blocked) shots for each pump pulse.

In the first case (CMOS), after passing through the choppers, the two reference beams are directed into the spectrometer using steering mirrors, and then focused onto a reference pixel on the detector using a lens - this reference pixel is placed outside of the probe spectrum to avoid probe fluctuations impacting the referencing intensity. The 
home-built Matlab code for this referencing process is shown in section C.1 of appendix B. In the second case, the PDA detector (conveniently) has a digital channel available in its data read out so a separate photo diode array can be used in place of focusing the reference beams onto the physical camera chip. Both CW reference beams are steered and focused from the choppers onto the PDA and the combined signal is electronically read out in the raw data file. The home-built Matlab code for this referencing process is shown in section B.4 of appendix B

For both cases, the chopper shots are sequenced post processing using the intensity of the two overlapped reference beams. The intensity of the reference signal can be used to then assign each shot post processing. Figure 4.7 shows how the intensity of the reference signal (either via intensity counts on a pixel or the PDA readout) can be used to then assign each shot post processing over a four shot sequence.

\subsection{Data processing}

\subsubsection{Wavelength calibration}

A solvent standard is used to calibrate the detectors x-axis, i.e to convert the spectral read out from pixel number to wavenumber. The standard chosen is acetonitrile as it is highly Raman active, and has many distinct, sharp Raman peaks across the vibrational regions of interest ${ }^{[125]}$.

For the spectrometer observing the $400-450 \mathrm{~nm}$ region, the Raman peaks of the solvent are measured and then, post processing, three main peaks present across the full energy range selected for a second-order polynomial fit. The pixel axis is converted to wavenumbers by first converting the Raman pump wavelength from its central wavelength (400 $\mathrm{nm}$ or $800 \mathrm{~nm}$ ) to be positioned as " $0 \mathrm{~cm}^{-1 \text { " }}$ and then calculating the Stokes' shift accordingly. The home-written Matlab code used in this calibration process is shown in B.3.

For the spectrometer observing the $800-1100 \mathrm{~nm}$ region, the available spectral range doesn't allow easy calibration using a standard, as the wavelength range on which the experiment is focused on didn't include three distinct peaks of the standard. To overcome this, three notch filters were used, specifically at $850 \mathrm{~nm}, 900 \mathrm{~nm}$ and $950 \mathrm{~nm}$. This allows the same second-order polynomial fit to convert between pixel number 
a

CMOS - acetonitrile
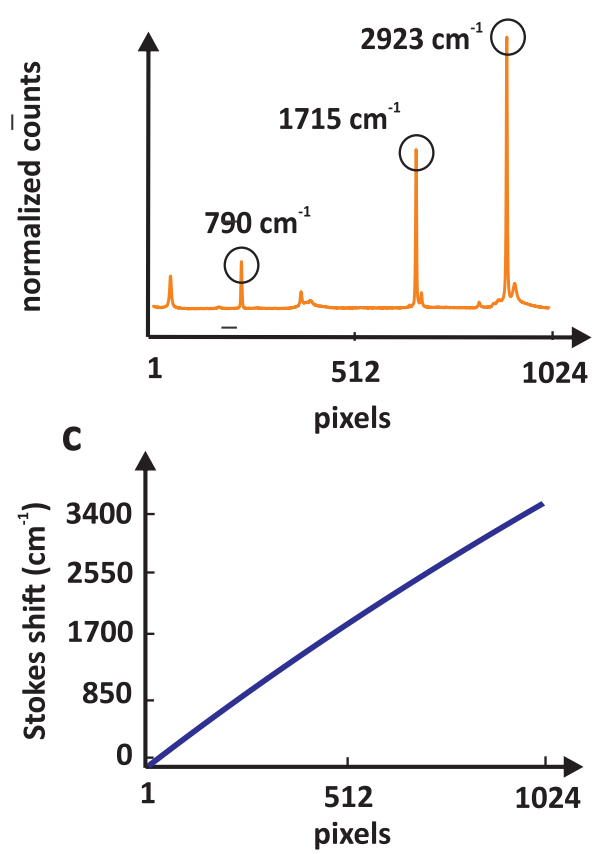

b

Stresing PDA - notch filters
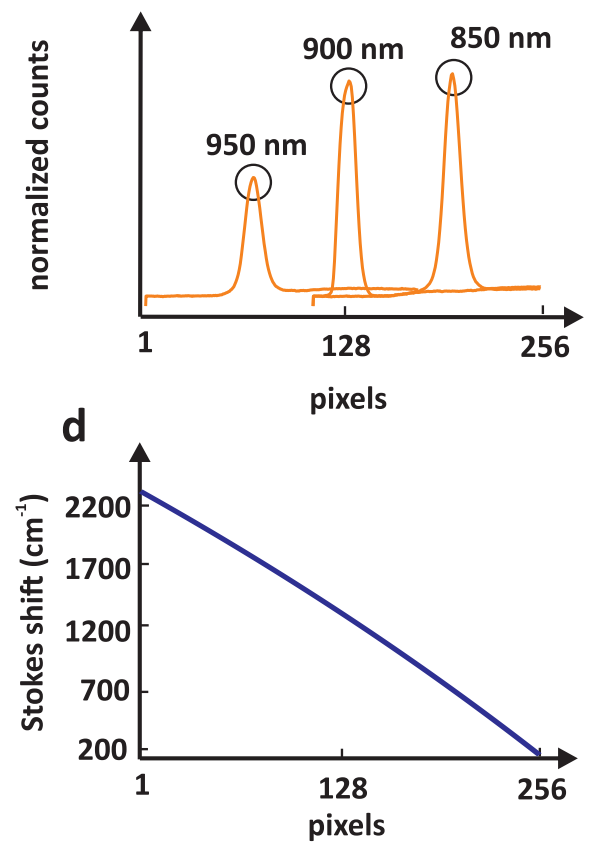

Figure 4.8: Calibration of each camera detector is performed fitting a second-order polynomial through three reference data points. Panels $a$ and $b$ show the three peak locations for the CMOS and PDA detectors respectively. A solvent standard is used in the case of the CMOS detector, while in the case of the PDA, three notch filters are used as the wavelength range doesn't allow for three sharp peaks using a standard. Panels $c$ and $d$ show the fit for each allowing conversion between pixel to wavenumber.

and wavenumber. An example of each fitting process is shown in figure 4.8. To ensure the notch filters are being read on the camera at the correct pixel locations (as, for example, having a notch filter on an erroneous angle, or in the focusing line of a beam, can laterally displace its position at the detector) the calibrated axis was tested using 2 peaks of the solvent standard. The home-built Matlab code for this process is shown in section B.4 of appendix B. 

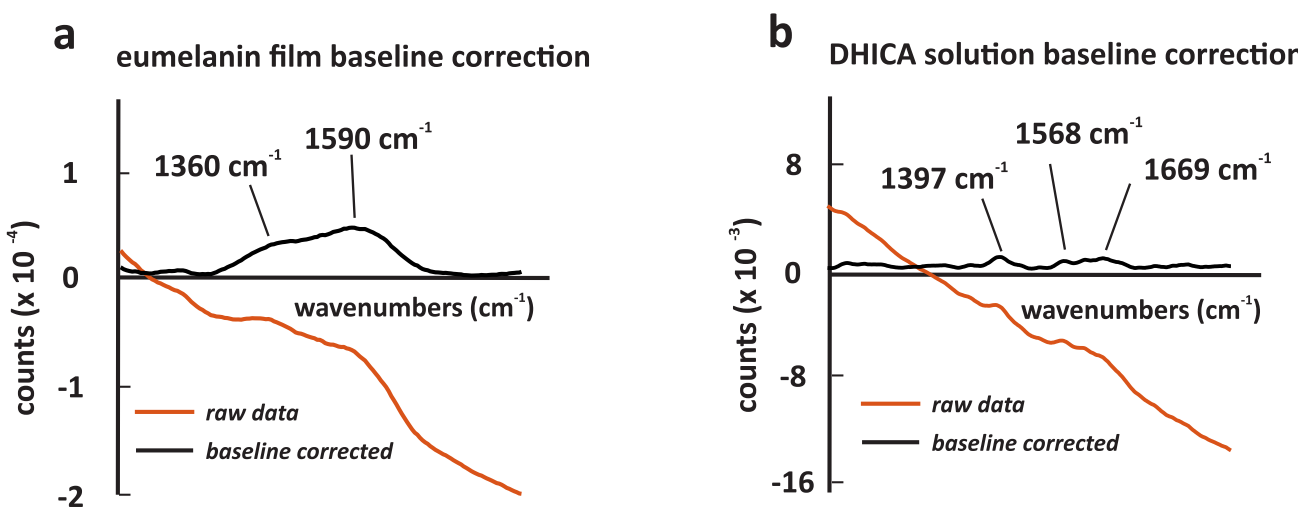

Figure 4.9: Two examples of automated baseline removal using an asymmetric least squares fitting algorithm. Panel $a$ shows the raw data for eumelanin film (in the ground state) in the orange curve, and the baseline removed data in the black curve. Panel $b$ shows the same for the ground state of DHICA in ammonia.

\subsubsection{Baseline removal}

Baseline issues plague FSRS experiments. These baselines arise due to cross phase modulation (CPM) between the Raman pump and the probe arriving at the same time at the sample, causing nonlinear effects. Typically in FSRS experiments, baselines are removed manually for each time slice which is both time consuming and potentially inconsistent. Additionally, removing baselines manually can be justified if the ground state Raman peak location are already known, as often the baselines are made up of many polynomials, especially if solvents are used.

As shown in figure 4.9, for eumelanin and DHICA, the baseline caused by CPM appears approximately linear, however even fitting a linear polynomial by hand to each time point post processing is laborious, and can introduce artefacts through human error. To overcome this, a baseline removal function using asymmetric least squares fitting is used to automate the removal of the baseline for each GS and ES time point before the calculation of the FSRS signal 126]127. The code used for this process was written by Professor Federico Marini, of the University of Rome La Sapienza and is provided in section B.5 of appendix B. The model estimates the baseline and then uses regression analysis to fit the baseline, using 3 parameters which are adjusted manually. The fit is then visually optimised by plotting each vector, with the same fit parameters being used for each time point for consistency. Figure 4.9 shows examples of the baseline removal for ground state Raman spectra of a eumelanin film (left panel) and 
DHICA in solution (right panel) for one time point. Typical values used for lambda, $p$ and maxit are set to $10^{4}, 0.01$ and 20 respectively.

\subsubsection{Solvent normalisation}

Where eumelanin, DHI and DHICA are measured in solution (as detailed in chapters 5 and 6), the solvent must be removed post processing, so that the solvent Raman peaks don't interfere with the material peaks. This is a time consuming process and one of the down sides to the FSRS method when looking at dilutions.

Solvent removal is achieved by first collecting experimental solvent only data, and processing it the same way as the solvent + material data, including averaging and baseline subtraction etc. A solvent peak is then normalised to the same solvent peak in the material data for each time point and subtracted. This is possible because the solvent doesn't change its intensity in the excited state, as the excitation pump is not absorbed. Fluctuations in the intensity of the solvent peaks are caused therefore only by Raman pump intensity fluctuations, so one advantage here is that, in effect, each time point is being intensity normalised to the other time points.

\subsubsection{Data processing}

Home-built Labview code (written in version 2015 SP1) is used to collect the experimental data for each time point for 28 time points over a $-3 \mathrm{ps}$ to $1 \mathrm{~ns}$ range. The software controls the camera readout, generation of the time steps on the electronic delay stage, photo-diode referencing readout and shot collection. The triggering of the detector's shot collection from the laser pulses, as well as the timing window for each shot, is also controlled using the software. The raw data files are saved as .dat files which are then processed following experimentation in home-built Matlab code. For each time point 300,000 shots are collected, with a typical experimental time of $\sim$ 1 hour. The outputted raw data files are imported into home-built Matlab code and converted into "pixel vs counts" matrices for all time points, with each row of a matrix being a single shot per time point. Each shot sequence is then extracted using the chopper referencing algorithm discussed in section 4.4.3. Once each shot in the four shot sequence is identified, four matrices are extracted per time shot, with each being 75,000 shots of the excitation and Raman pump shot sequences as shown in figure 4.10 . 
step 1: shot selection

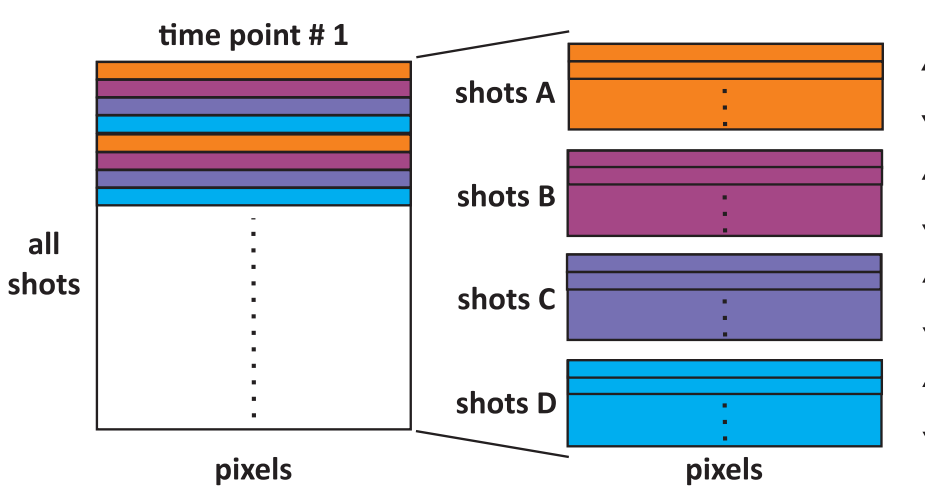

step 2: shot averaging

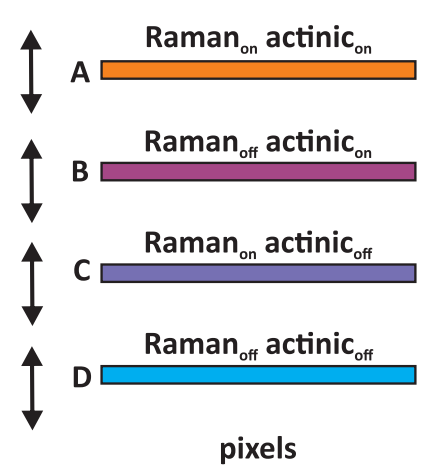

signal calculation

transient absorption $=$ B - D
ground state Raman $=$ C - D
excited state Raman $=$ A - B

Figure 4.10: The left panel shows the first step in the data processing analysis - the importation of the dat file as a matrix of pixels ( $x$-axis) versus shots ( $y$-axis). Once the shot referencing has be completed, each shot can then be pulled from the matrix to make four new matrices, shown in the middle panel. Then, they can be averaged and the vectors used to calculated each of the signals: transient absorption, ground state Raman and " excited state" Raman (i.e excitation pump is "on", shown in the right panel. Following this, the calculation of the Raman gain signal (FSRS) can be processed.

Following shot extraction, each of the four matrices are averaged along the shot axis, producing four vectors $A, B, C$ and $D$ for each of the 30 time points. By combining each vector as shown in figure 4.10, the transient absorption (TA), ground state Raman (GS) and excited state Raman (ES) (excitation pump "on"), signals can be calculated. Each of the 30 GS and ES vectors are then baseline corrected as discussed in section 4.5.2. The pixel axis is then calibrated as discussed in section 4.5.1. Following baseline removal and axis calibration, the shots taken before time-zero (that is, no excitation pump) are averaged and removed from the GS and ES vectors. Each GS and ES vector for each time point are then meshed into two matrices of wavenumbers $\left(\mathrm{cm}^{-1}\right)$ vs time points ( $p s)$, and the FSRS surface is calculated. 
The time points axis is calibrated by plotting the spectrally averaged kinetics of the TA data (in the case of eumelanin, this is the rise of a photo-induced absorption (PIA) band) to ensure "time-zero" is assigned correctly on the delay stage. It is observed in the TA surfaces that no rigorous chirp correction is required, due to the narrow spectral range $(50 \mathrm{~nm})$ on the detector and low temporal resolution.

Various noise reduction algorithms were tested to improve the SNR of the final signal, including mean-averaging and median-averaging based on the elimination of outlying data points with a $>3 Z$-score for each data stack ${ }^{[128}$. However, it is found that this is computationally time consuming and did little to improve the actual SNR of the GS and ES signals and that, instead, by experimentally averaging over several thousands of shots, the SNR is greatly improved. Once the FSRS signal is extracted a 3-point moving mean across the energy axis is applied to counter pixel noise. The extraction of each signal, GS, ES, TA and FSRS is written into the same Matlab function as the chopper sequencing code as shown in appendices C.1 and B.2 and presented as a 3D surface of wavenumbers vs time points vs Raman gain.

\subsubsection{Data analysis}

\subsubsection{Singular value decomposition (SVD) analysis}

SVD is a mathematical method that allows the factorisation of a matrix into its primary components [129-131]. By factorisation, the isolation of specific excited state spectra, and their corresponding kinetics, from the FSRS surface is achieved. The FSRS surfaces are inputted as " $x-y-z$ " data matrices, in this case as "wavenumber - time - Raman gain intensity". Each matrix is factorised into spectral and temporal components, allowing the identification of specific spectral species and their corresponding kinetics. By applying a temporal mask vector to the SVD analysis using the TA kinetics collected in the same experimental conditions, excited state features can be isolated from any noise or baseline correction errors that may have contaminated the dynamics. A Matlab MCR toolbox ${ }^{[132]}$ is used to process SVD spectra and kinetics for all samples. Chapters 5 and 6 discuss details of the specific SVD analysis carried out for DHI, DHICA and eumelanin films and solutions. 


\subsubsection{Density function theory (DFT) analysis}

Density functional theory (DFT) is a computational method used to model the electronic and nuclear structure of molecules based on the structure of the molecule ${ }^{[133]}$. In DFT the the Born-Oppenheimer approximation is used, where the nuclei are treated as fixed relative to the electrons ${ }^{[133[134]}$. The electron density is used to approximate the system's wave function, by making an initial estimate of the electron's cross correlation function $(\mathrm{CCF})$, that is, how the electrons interact together, and then by iterating that function to close in on the minimum potential energy well surface, " $E$ ". The electron density in this way defines the potential energy function, or potential energy surface (PES) for nuclear motion. At a minimum energy geometry (in terms of the spatial arrangement of nuclei), we can extract the vibrational frequencies of the molecule from the curvature of the PES as the normal modes. Here, we are treating each vibration as if it were a quantum-mechanical simple harmonic oscillator, a reasonable approximation given the system's fast vibrational relaxation time.

A molecule with $N$ atoms has $3 N$ coordinates and $3 N-6$ vibrational modes ${ }^{[135]}$. The PES (ignoring spin) is $3 N$ dimensional, where the energy depends on the $x, y$ and $z$ coordinates of each nucleus/atom. 3 of these dimensions correspond to translational motion in each of the $x, y, z$ directions, and 3 more correspond to rotations about the $x, y, z$ axes. The remaining $3 N-6$ dimensions define motions in which the positions of the nuclei change with respect to each other, i.e their vibrations.

In the experiments presented in this chapter, and ground state DFT models presented in subsequent chapters, the DFT analysis was performed using the Gaussian 09, Revision D.01 programme, with computations being conducted by Paul Hume of the Victoria University of Wellington.

It should be noted that at the time of analysis, DFT software to calculate ground state frequencies only was available for the "system-build testing" of the FSRS system. Hence the analysis presented in the following test section of napthalene, section 4.6, comparisons with DFT calculations to the experimental data are presented in the ground state only. However in the subsequent experimentation and analysis of eumelanin and its monomers, presented in chapters 5 and 6 , access to DFT software capable of calculating excited state vibrational modes was also available, that is Gaussian 16, Revision C.01. The DFT method selected for the excited state analysis in these cases can be justified as follows: 
- The functional/basis set combination B3LYP/6-31G(d) has previously been shown to provide predicted Raman spectra in close agreement with the experimentally measured ground state Raman spectrum of DHICA ${ }^{[136]}$.

- Our method uses the long-range corrected version of B3LYP, CAM-B3LYP, with a larger basis set, and a solvent model, and provides comparable predictions for the ground state Raman spectrum of DHICA.

- We expect our method to provide a better description the excited state Raman spectra for several reasons:

- Previous theoretical work on DHI implicates the population of very diffuse molecular orbitals, leading to proton-coupled electron transfer ${ }^{[137]}$. Describing the electron distribution in these states therefore requires a basis set including diffuse functions (hence the selection of $6-31+G(d, p)$ ), and a functional capable of describing long-range Coulombic interactions (hence the selection of CAM-B3LYP). Note: this work finds CAM-B3LYP in combination with polarisable continuum solvation to provide reasonable predictions of excitation energies compared to more rigorous MS-CASPT2 calculations.

- The formation of anionic intermediates due to either proton transfer, or proton-coupled electron transfer also necessitates the use of a basis set capable of describing delocalised electron distributions.

- Formation of anionic intermediates also necessitates the use of a solvent model to describe the stabilisation of the charges by the surrounding solvent.

DFT calculations of the excited state were performed using Gaussian 16, Revision C.01 using the CAM-B3LYP exchange-correlation functional in combination with the $6-31+\mathrm{G}(\mathrm{d}, \mathrm{p})$ basis set. All calculations employed an ultrafine integration grid (i.e. a mesh defined by 99 radial shells and 590 angular points per shell for each atom) with two-electron integrals calculated to an accuracy of 10-11. Geometry optimisations employed tight convergence criteria, and minima confirmed by inspection of the normal mode vibrational frequencies to confirm the absence of imaginary frequencies. The effect of the aqueous medium was treated using a polarisable continuum model (integral equation formalism) using the default parameters for water. 

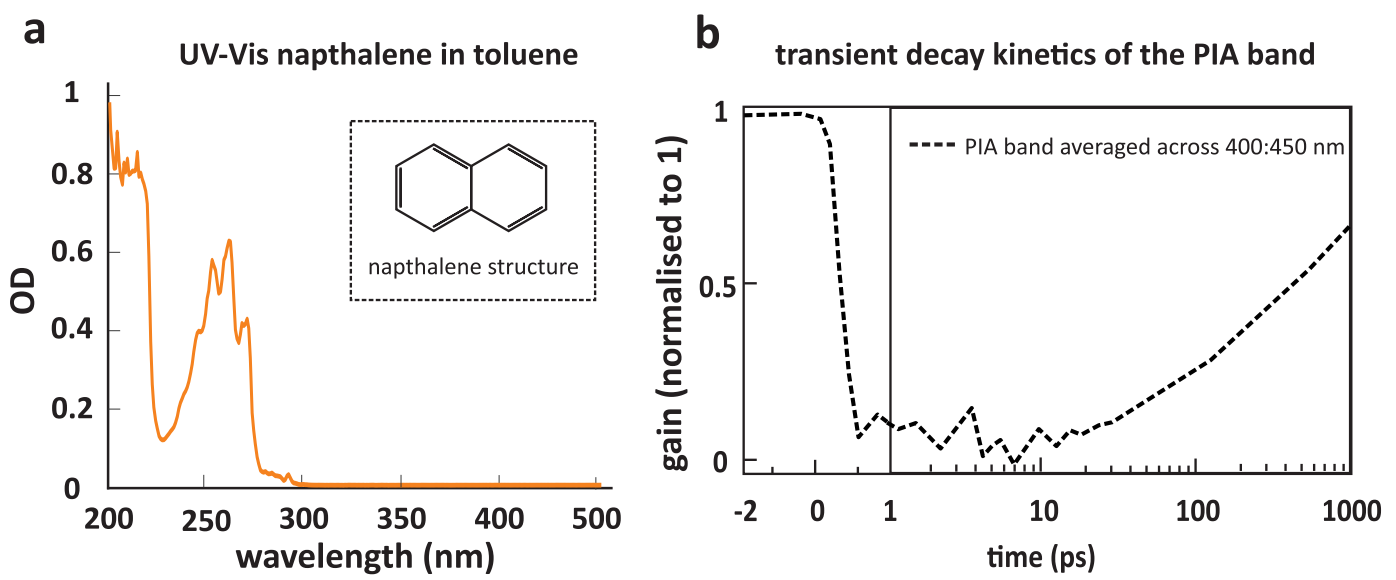

Figure 4.11: Panel $a$ shows the UV-Vis absorption of napthalene in toluene solution, with a strong absorption at $267 \mathrm{~nm}$ of $\sim 0.5$ OD and no absorption in the visible region, specifically at $400 \mathrm{~nm}$ to which the Raman pump is tuned. The PAH structure of napthalene is shown in the inset of this panel. Panel $b$ shows the transient absorption kinetics averaged across a 400 to $450 \mathrm{~nm}$ spectral range, showing a strong excited state PIA band in this region. This plot is normalised so that the gain $=1$ at negative times (pre- time-zero).

\subsection{FSRS system test - napthalene}

\subsubsection{Material background photophysics}

Napthalene is a polycyclic aromatic hydrocarbon (PAH) made up of two fused benzene rings with the formula $C_{10} H_{8}{ }^{[138}$. Its structure is shown in panel $a$ of Figure 4.11 , and its UV-Vis absorption spectrum shown in panel $c$ of the same figure. Napthalene was chosen as a test sample for the FSRS build system because of its electronic properties the transient absorption spectrum of napthalene absorbs in the UV at around $267 \mathrm{~nm}$ and has a photo induced absorption band at $400 \mathrm{~nm}$ [139-141]. This is the ideal for testing the FSRS set up using a $267 \mathrm{~nm}$ excitation pump, as the material will create an excited state population upon absorption of the UV, and then achieve resonance enhancement with the Raman pump at $400 \mathrm{~nm}$ due to the PIA band. 


\subsubsection{Experimentation and results}

\subsubsection{Sample preparation}

Napthalene is prepared as a solution in toluene, at the highest concentration possible, achieving an OD of 0.5 at $267 \mathrm{~nm}$. The excitation pump at $267 \mathrm{~nm}$ had a pulse energy of $0.6 \mu \mathrm{J}$, while the Raman pump at $400 \mathrm{~nm}$ had a pulse energy of $1 \mu \mathrm{J} .30$ time points were collected averaging 60, 000 shots per time point.

\subsubsection{Analysis of ground state modes}

Figure 4.12 shows the main 6 ground state peaks for napthalene collected experimentally compared with DFT analysis. The vibrations associated with each frequency are shown graphically in panel $a$ of the figure as the blue cure, the orange lines representing the calculated frequencies from DFT analysis. An exact comparison of the experimental and calculated frequencies are presented in the chart in panel $b$. The experimental ground state peaks also show good agreement with literature ${ }^{[142]}$. The physical vibrations associated with each of the 6 main peak frequencies are presented graphically in figure 4.13 .

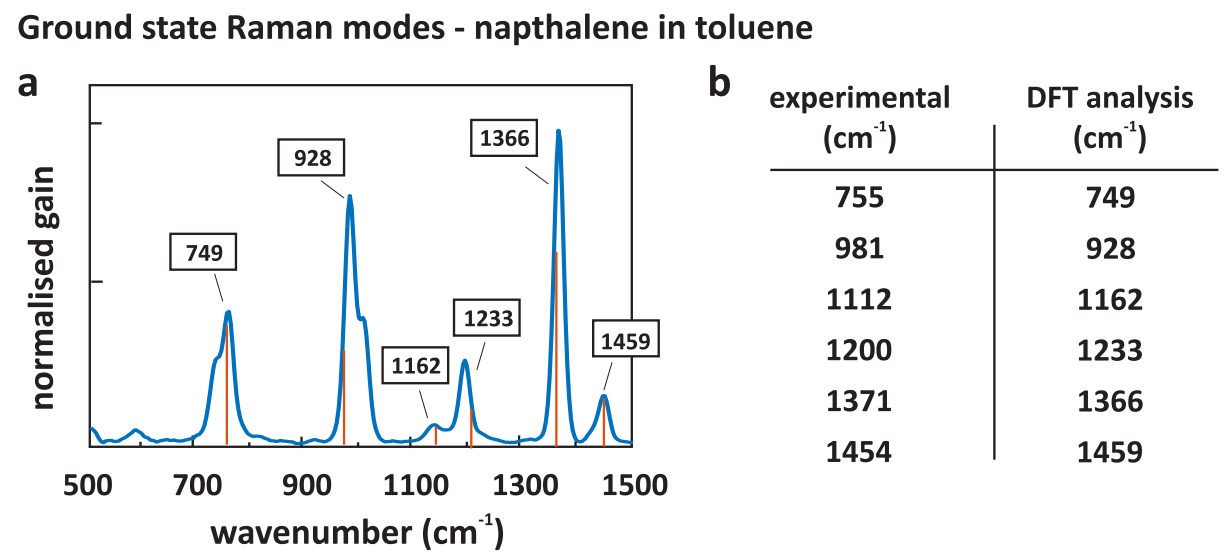

Figure 4.12: The experimental ground state Raman modes of napthalene as shown in panel $a$. The blue curve is data from the FSRS experiment, while the orange lines indicate the frequency positions of naphthalene's ground state modes from DFT calculations. Panel $b$ shows the experimental mode peaks compared to the DFT calculated mode peaks. This DFT analysis was performed by Paul Hume of the Victoria University of Wellington using Gaussian 09, Revision D.01. 
DFT mode assignments for napthalene (ground state)

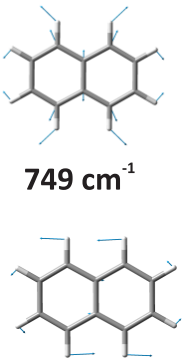

$1233 \mathrm{~cm}^{-1}$

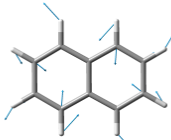

$928 \mathrm{~cm}^{-1}$

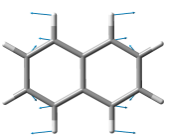

$1366 \mathrm{~cm}^{-1}$

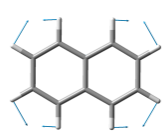

$1162 \mathrm{~cm}^{-1}$

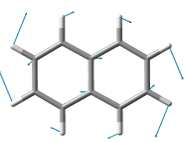

$1459 \mathrm{~cm}^{-1}$

Figure 4.13: The calculated ground state vibrational energies and mode assignments for napthalene using density functional theory (DFT) analysis are shown in panels $a$ and $b$. The key ground state modes are shown, with their frequencies in good agreement with those obtained from the FSRS experiment. This DFT analysis was performed by Paul Hume of the Victoria University of Wellington using Gaussian 09, Revision D.01.

\subsubsection{Excited state FSRS analysis of napthalene}

The left hand panel of figure 4.14 show the FSRS surface generated post-processing, and the evolution of the Raman peaks for 15 time slices is shown in the right hand panel (displaced for clarity), with the ground state shown as the black curve at the bottom of the figure. The evolution of dynamic Raman activity is apparent and can be seen within the $1000 \mathrm{ps}$ time range. At early times, dispersive modes can be seen in the excited state surface, indicating that the excited state Raman vibrations are close in frequency to the ground state. Of particular interest is the fast vibrational relaxation shown near the $980 \mathrm{~cm}^{-1}$ and $1370 \mathrm{~cm}^{-1}$ modes, where the dispersive curves in the excited state narrow within $0.4 \mathrm{ps}$. This indicates a cascaded vibrational relaxation in the excited state, as the narrowing dispersive curve means that the excited state modes are becoming closer in frequency to the ground state modes.

This evidence of dynamic Raman activity, along with the corresponding transient absorption data providing evidence of an excited state population, concludes that the FSRS system is working as expected. 

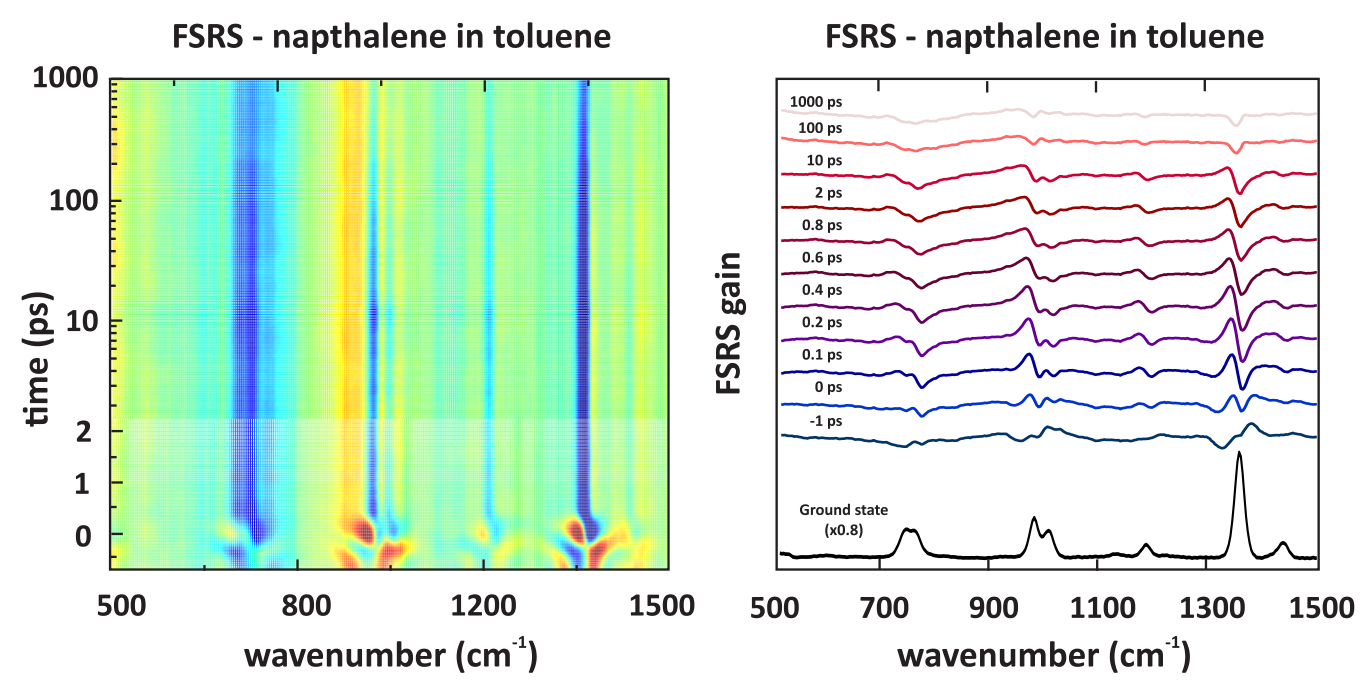

Figure 4.14: The FSRS dynamics of napthalene in toluene, are shown first as a surface in the left hand panel, for a time range of $1000 \mathrm{ps}$ (y-axis) across a 500 to 1500 $\mathrm{cm}^{-1}$ energy range. The right hand panel shows time slices (displaced along the $\mathrm{y}$-axis for clarity) revealing relatively fast, and dispersive dynamics, with complete relaxation by about $100 \mathrm{ps}$. The ground state Raman spectrum for napthalene is shown in black.

\subsection{Chapter summary}

In this chapter the build of the FSRS system has been outlined. This includes the system requirements, design, experimental processing and testing using a standard material. The key component of the system build are as follows:

- The experimental configuration of the FSRS system is presented, which each key optical component of the system build introduced. The Ti:sapphire amplifier light source is split into 3 beam lines to generate the 3 laser pulses required for FSRS, as well as the electronic delay line to achieve dynamic resolution, the mechanical choppers used to achieve appropriate pulses sequencing, the sample area and the home built spectrometer for recording Raman gain and transient absorption spectra.

- The three beam lines required to generate the FSRS laser pulses are presented:

- The existing TA system in our lab was modified to include a third laser pulse, the Raman pump, using a novel technique for bandwidth compression and a classical spatial filtering method, producing Raman pump pulses 
in the visible $(400 \mathrm{~nm})$ and IR $(800 \mathrm{~nm})$.

- A second and third harmonic generator system is presented to produce the excitation pump in the UV (267 $\mathrm{nm})$ and visible (400 $\mathrm{nm}$ ) regions. was built.

- The supercontinuum white light probe is generated by focusing a portion of the fundamental laser pulse in YAG and $\mathrm{CaF}_{2}$ nonlinear crystals, to produce probe pulses with the appropriate spectral ranges in the UV-visible and visible-IR regions.

- Improvements to the optical build of the system in terms of the beam mode and quality of the probe laser pulse are presented

- The build of two high resolution spectrometers in the Czerny Turner grating configuration to capture Raman spectra across $400 \mathrm{~nm}-450 \mathrm{~nm}$ and $800 \mathrm{~nm}$ to $1100 \mathrm{~nm}$ spectral ranges respectively, including the calculations made to ensure the optical components of each allow for sufficient optical and digital resolution.

- New software was written in Matlab to process the raw FSRS data (output in Labview) including data input, shot stack averaging, axis calibration, chopper sequencing, solvent removal, baseline removal and extraction of the ground state Raman gain, transient absorption spectra and kinetics, and the FSRS data surfaces.

- An introduction into post-processing data analysis using SVD and DFT is presented.

- The system was tested using napthalene in toluene which allowed testing of each of the critical elements in this build, particularly the post-processing software. Comparisons with literature showed good alignment with the ground state Raman modes and transient absorption kinetics. Additionally DFT calculations were bench-marked against the ground state Raman modes, validating both the FSRS experiment and the choice of DFT variables. Napthalene's FSRS dynamics are within the expected lifetime of the excited state, given by the transient absorption kinetics, and shows dispersive peaks narrowing over a $<p s$ timescale, an indication of excited state vibrational relaxation.

The FSRS system has a spectral resolution of $20 \mathrm{~cm}^{-1}$ and a temporal resolution of $100 f s$ in the limit of the instrument response function (IRF). Further improvements can be made in future work to increase the resolution of the FSRS system, particularly in terms of the optical resolution, which is limited currently to about $20 \mathrm{~cm}^{-1}$ in the 
visible, and $39 \mathrm{~cm}^{-1}$ in the IR. Further modifications to the bandwidth compressor could achieve the generation of a Raman pump using the bandwidth compressor with sufficient intensity to be used in the IR, without the need to incorporate the secondary bandwidth compression method of spatial filtering. 


\section{Chapter 5}

\section{Spectroscopic signatures of eumelanin subunits}

\subsection{Introduction}

Di-hydroxy-indole (DHI), and its carboxylated form 5,6-di-hydroxyindole-carboxylic acid (DHICA), are well agreed to be the key building blocks of eumelanin pigment. 22166[192526] The DHI and DHICA subunits of eumelanin have been previously studied in detail, particularly in terms of their photoluminescence (PL) activity on ultrafast timescales. . $2045546499511431144^{2}$ Previously conducted time-dependent PL measurements indicate that excited state proton transfer (ESPT) could describe energy dissipation mechanisms in DHICA upon light excitation ${ }^{45446}$.

Both DHI and DHICA monomers rapidly polymerise in air, and under photo-excitation, and so studies into these materials are inherently challenging, particularly under high intensity pulsed laser systems. By studying the structure and ultrafast dynamics of these eumelanin subunits using a combination of FSRS and transient absorption spectroscopy, we hope to gain insight into eumelanin pigment itself, particularly by comparing the spectral responses and kinetics of each material under the same experimental conditions. The studies presented in this chapter are used as references in the assignment of eumelanin's key vibrational species, which is discussed in chapter 6.

This chapter is set out as follows:

- First, a summary of the current energy dissipation models for DHI and DHICA are presented, as introduced in chapter 1 , with a specific focus on excited state 
proton transfer (ESPT) of the DHICA monomer in neutral $\mathrm{pH}$ solvent conditions.

- Secondly, two experimental configurations used for the FSRS experiments is presented, including sample preparation for each material, and the two excitation and Raman pump wavelength configurations used.

- Thirdly, the experimental FSRS results are detailed, including the vibrational and electronic optical properties observed in DHI and DHICA upon photo-excitation, and their photo-induced excited state relaxation dynamics.

- Fourthly, data analysis of the FSRS experiments is presented and discussed for DHI and DHICA along with a density functional theory (DFT) analysis for their vibrational mode assignments. Here, evidence for excited state proton transfer (ESPT) in DHICA is presented, with the observation of dynamic vibrational peak frequency shifting in the FSRS experimental data compared with that of DFT analysis.

- Finally, a comparison of the vibrational dynamics of DHICA dissolved in two different solvents is presented, with results being in support of ESPT as being a key energy dissipation pathway for DHICA.

\subsection{Energy dissipation models}

The molecular structures of DHI and DHICA are shown in figure 5.1. Here we see the variation between the two subunits being the carboxylic acid $(\mathrm{COOH})$ group present in DHICA. The $\mathrm{COOH}$ site allows for both inter-molecular proton transfer between the $\mathrm{H}$ of the $\mathrm{COOH}$ group to the solvent bath, after which DHICA is a deprotonated anion (COO-), due to the $\mathrm{COOH}$ group being negatively charged. The phenol groups present in both DHI and DHICA structures therefore become the relevant site for deprotonation in the excited state, and can subsequently form anions and di-anions via inter-molecular deprotonation to the solvent at neutral $\mathrm{pH}$. The $p K_{a}$ value for an $\mathrm{OH}$ phenolic group is 10.0 , while that of a carboxylated COO- acid group is 3.75 , meaning an equilibrium will become established for the $\mathrm{COOH}$ group via deprotonation to the solvent in the dissolution of the material in water, while intra-molecular proton transfer between the phenol and COO- groups will be prohibited ${ }^{[145]}$. 


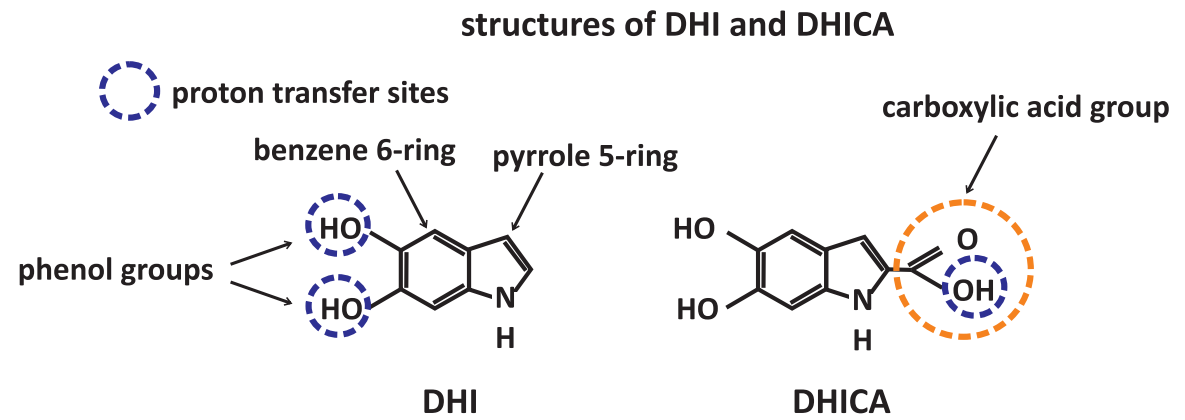

Figure 5.1: The structures of DHI and DHICA are shown with the key sites identified. The phenol $(\mathrm{OH})$ groups present in both molecules, and the carboxylic acid $(\mathrm{COOH})$ site in DHICA are capable of proton transfer as a mechanism for energy dissipation. In DHICA, the $\mathrm{COOH}$ can undergo intra-molecular proton transfer to the $\mathrm{NH}$ site of the pyrrole ring, as well as inter-molecular proton transfer to the solvent at a neutral $\mathrm{pH}$. Both monomers can transfer protons to the solvent from their phenol groups, in appropriate solvation conditions. In the case of dimers and trimers that can be formed from these monomers, it is also possible that the deprotonated phenoxylate groups can then scavenge protons lost to the solvent to secondary phenol groups in dimers and trimers.

Excited state proton transfer (ESPT), introduced in chapter 1, has been proposed as the primary relaxation mechanism for DHICA under neutral and low $\mathrm{pH}$ solvent conditions ${ }^{[55[50146]}$. As mentioned, there are three available sites within DHICA for proton transfer to occur, and two in DHI. In both DHI and DHICA, inter-molecular proton transfer can occur between the two phenol groups $(\mathrm{OH})$ of the benzene ring to the solvent bath. In DHICA, intra-molecular proton transfer can also occur between the carboxyl $(\mathrm{COOH})$ group and $\mathrm{NH}$ site of the neighbouring pyrrole ring. When dissolved in a solvent at $\mathrm{pH} 7$ (e.g. water) the protons of the carboxylic acid group in DHICA will be mostly lost to the solvent, so the material will primarily be present in its deprotonated form in the ground state. Once the DHICA monomer forms a carboxylate group, the size of the molecule prohibits recombination between the positive phenol groups and negative carboxylate site - this means any further proton transfer upon excitation must come from the two phenol groups to the solvent.

Upon excited state deprotonation of the phenol groups, the di-anion then relaxes by fluorescing, and by scavenging protons back from the solvent the molecule returns to its ground state. This mechanism is shown in figure 5.2. Further studies of dimers 


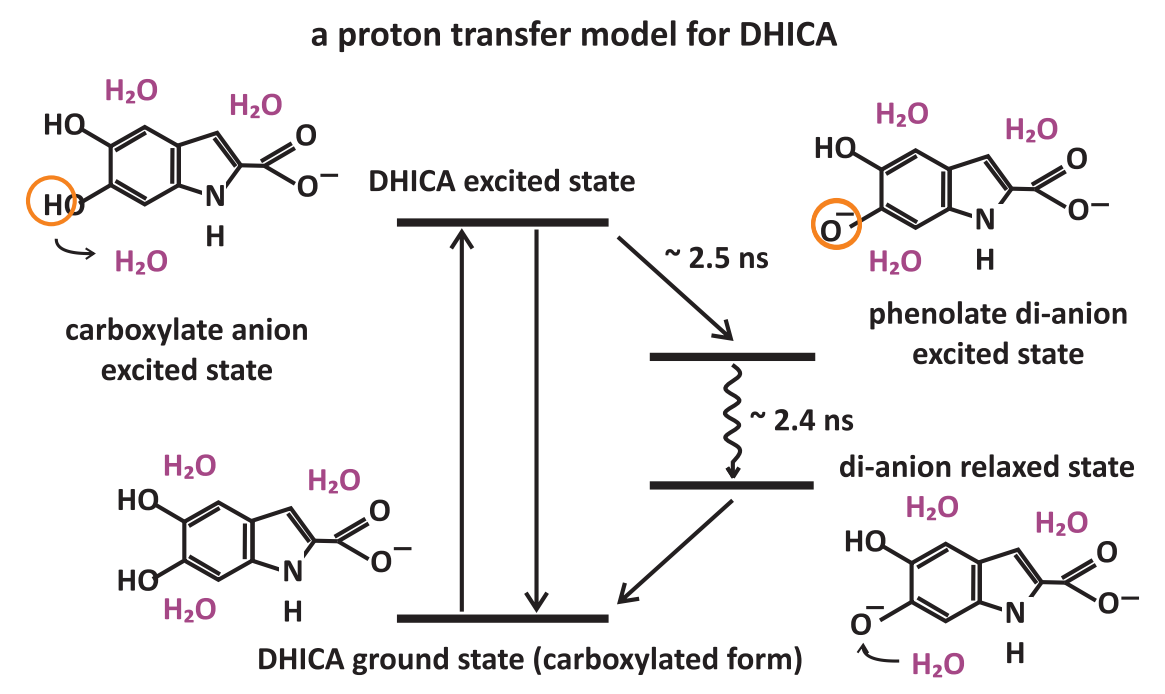

Figure 5.2: Proton transfer model for the DHICA monomer, where the relaxed sate in an anion caused by the loss of the $\mathrm{H}+$ in the carboxylic acid to the solvent upon dispersion in water. Upon excitation, the phenol groups can lose protons to the solvent causing a rapid relaxation to a di-anion excited state. Further relaxation via fluorescence on a $n s$ time scale allows for scavenging protons back from the solvent to return to its ground state.

and trimers of DHICA would be interesting, as the close bonding proximity of each monomer could facilitate a rapid back-and-forth inter-molecular exchange of protons within the bonded molecule via the solvent.

Proton-coupled electron transfer (PCET) is a second model proposed for energy dissipation introduced in chapter 11. In this case, excited state charge transfer (CT) to an electron acceptor, is synchronised with proton transfer to the solvent ${ }^{455152]}$. This mechanism supports the $\pi$ stacking structural system proposed for eumelanin, discussed further in chapter $6^{[20]}$.

Here, in our study of DHI and DHICA and their excited state vibrational dynamics, we aim to reveal the energy dissipation mechanisms which can provide insights into the overall structure and rapid energy dissipation of the eumelanin macro-molecule. The focus of our research is primarily to unravel the energy dissipation mechanism of eumelanin itself, and by providing a reference for the spectral signatures of its monomer subunits, we aim to be closer to that goal. By comparing the non-radiative dissipation 
mechanisms eumelanin's subunits, insights into eumelanin's relaxation pathways are established. Testing the leading hypothesis of ESPT as an energy dissipation mechanism for DHICA and the eumelanin macro-molecule is the focus of this chapter [146].

\subsection{Experimental configuration}

\subsubsection{FSRS experimental configurations}

Two separate experimental configurations are used in the FSRS experiments of DHI and DHICA presented in this chapter. Raman pump wavelengths are used in the IR at $800 \mathrm{~nm}$ and in the visible at $400 \mathrm{~nm}$. Two solvents preparations, distilled (de-gassed) water and (de-gassed) methanol, are used in the sample preparations of DHICA, for the visible Raman pump, while de-gassed water is used as the solvent for both DHI and DHICA for the IR Raman pump configuration. These experiments will be referred to hereafter as configuration 1 and configuration 2, with table 5.1 summarising each experimental configuration.

\begin{tabular}{|l|l|l|l|}
\hline \multicolumn{3}{|c|}{ Experimental configurations for DHI and DHICA FSRS experiments } \\
\hline \hline configuration & sample preparation & Raman pump details & excitation pump details \\
\hline 1 & DHI in water & $800 \mathrm{~nm}, 340 \mu \mathrm{J} / \mathrm{cm}^{2}$ & $267 \mathrm{~nm}, 170 \mu \mathrm{J} / \mathrm{cm}^{2}$ \\
& DHICA in water & $800 \mathrm{~nm}, 340 \mu \mathrm{J} / \mathrm{cm}^{2}$ & $267 \mathrm{~nm}, 170 \mu \mathrm{J} / \mathrm{cm}^{2}$ \\
\hline 2 & DHICA in water & $400 \mathrm{~nm}, 280 \mu \mathrm{J} / \mathrm{cm}^{2}$ & $267 \mathrm{~nm}, 170 \mu \mathrm{J} / \mathrm{cm}^{2}$ \\
& DHICA in methanol & $400 \mathrm{~nm}, 280 \mu \mathrm{J} / \mathrm{cm}^{2}$ & $267 \mathrm{~nm}, 170 \mu \mathrm{J} / \mathrm{cm}^{2}$ \\
\hline \hline
\end{tabular}

Table 5.1: Experimental configurations for FSRS experiments conducted on DHI and DHICA. In configuration 1, the two monomers are prepared in de-gassed water, with an IR Raman pump at $800 \mathrm{~nm}$, while in configuration 2, an visible Raman pump at $400 \mathrm{~nm}$ is used for DHICA only in 2 different solvent preparations.

The different configurations serve different purposes in the FSRS experiments. When observing the DHI monomer, rapid polymerisation can be seen in the transient absorption data (refer to figure 5.4) which shows a PIA peak around $1.7 \mathrm{eV}$, close to eumelanin's PIA band. Degradation can also be observed in the UV-Vis spectra of the dilution as shown in figure 5.3, when comparing pre- and post- FSRS experiment UV-Vis absorption. Additionally, any polymerisation present in the DHI and DHICA 
monomers will be resonantly enhanced in the FSRS spectra using a Raman pump in the IR. It was therefore decided for subsequent experiments to observe potential ESPT by comparing solvent dependence that DHICA only would be studied, with a Raman pump in the visible region at $400 \mathrm{~nm}$. While the results of the data obtained for DHI and DHICA in configuration 1 are likely that of oligomers, not pure monomers, the data is still presented here for interest.

\subsubsection{Sample preparations}

For configuration 1, DHI (AK Scientific; Z1653, 3131-52-0) and DHICA (AK scientific; 6559AB, 4790-08-3) are prepared in distilled water to achieve saturation, specifically $5 \mathrm{mg}$ of each powder being dissolved in $1 \mathrm{~mL}$ of the solvent. The water is first degassed using 4 iterations of the freeze-pump-thaw method under argon ${ }^{147}$. The DHI and DHICA powders were initially prepared in non-degassed water, but were found to immediately polymerise and form aggregates on the surface of the cuvette under exposure to the pulsed laser. To obtain sufficient concentration, the powders are dissolved at the highest possible saturations, and then sonicated for 30 minutes. The prepared dilutions are syringe filtered into a $1 \mathrm{~mm}$ thick cuvette to remove un-dissolved aggregates and to obtain scatter free dilutions for experimentation. Absorptions of $0.163 O D$ and $0.82 O D$ for DHI and DHICA respectively (as shown in figure 5.3) are obtained at $267 \mathrm{~nm}$ excitation using this method. With an approximate extinction coefficient value of $5000 \mathrm{~cm}^{-1} / M$ for both materials at $267 \mathrm{~nm}{ }^{[148]}$, concentrations of $0.000326 \mathrm{~mol} / \mathrm{L}$ (for DHI) and $0.001136 \mathrm{~mol} / \mathrm{L}$ (for DHICA) are achieved - sufficient to create an excited state population while maintaining a high SNR for measurement of the ground and excited state Raman gains.

For configuration 2, DHICA (AK scientific; 6559AB, 4790-08-3) powder is dissolved in two separate solvent preparations, distilled water and methanol. The solvents are each prepared using the freeze-pump-thaw method as for configuration 1, with $5 \mathrm{mg}$ of powder being dissolved in $1 \mathrm{~mL}$ of each solvent. The purpose of this experimental configuration is to compare the FSRS dynamics of the DHICA monomer, where in water ESPT is allowed, while in methanol it is prohibited ${ }^{451}$. For the neutral water solvent, the carboxylic acid group in DHICA is deprotonated in the ground state, and ESPT can occur from the phenol groups of the anion to the solvent upon photo-excitation. However, in methanol DHICA cannot transfer protons to the solvent, and so this pathway is prohibited. Prepared samples have optical absorptions of $0.85 O D$ and $1.5 O D$ for 
a

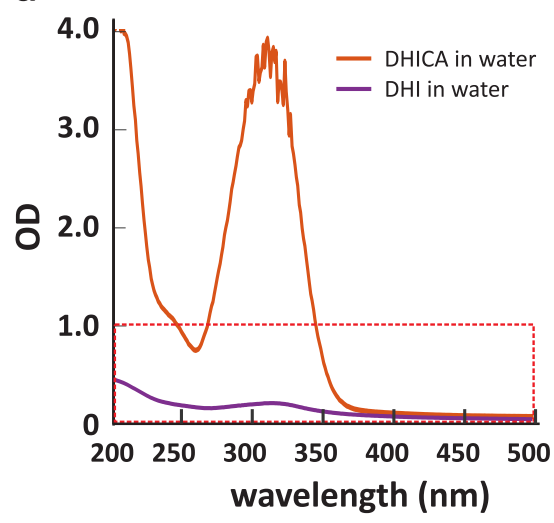

b

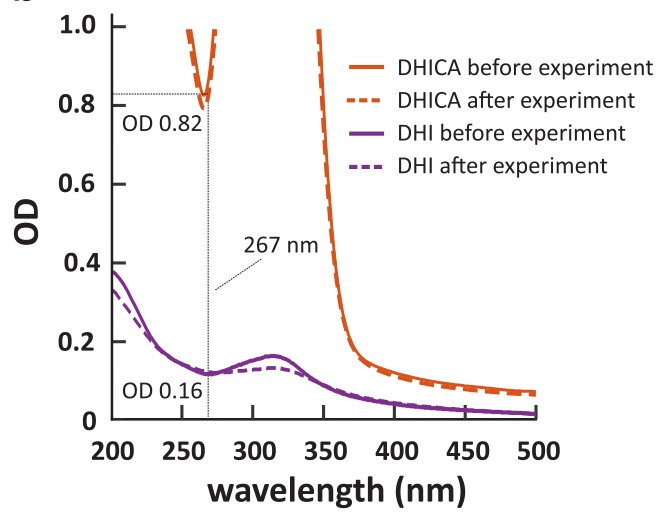

Figure 5.3: The UV-Vis absorptions of the DHI and DHICA monomers dissolved in distilled (de-gassed) water are shown in panel $a$; the red dashed box in this figure indicates the zoomed in region shown in panel $b$ with the OD for each material noted for $267 \mathrm{~nm}$ as 0.163 and 0.82 respectively. These optical densities are suitable to establish an excited state population for the FSRS experiment using a 267 $n m$ excitation pump. The dashed curves in panel $b$ show the UV-Vis for the same samples taken after experimentation, with the DHI absorption spectrum indicating a small sample degradation. This is likely due to photo-induced polymerisation in the dilution, but the high averaging of shots means that any degradation should average out across the measurement, rather than have a dynamic trend.

DHICA in water and methanol respectively.

All prepared dilutions are mounted onto a home-built raster stage for the FSRS experiment which continuously translates the cuvette "side-to-side", perpendicular to the incoming laser beams in order to prevent excited state build up and polymerisation upon laser excitation. A magnetic stirring rod is deposited into the cuvette and an external home-built rotating magnet causes this rod to continuously stir the dilution in conjunction with rastering the cuvette.

\subsubsection{Excitation pump configurations}

In configuration 1, fluences of $\sim 170 \mu \mathrm{J} / \mathrm{cm}^{2}$ and $\sim 340 \mu \mathrm{J} / \mathrm{cm}^{2}$ are used for the $267 \mathrm{~nm}$ excitation pump, and $800 \mathrm{~nm}$ Raman pump respectively. The fluences are calculated by measuring each beam's spot sizes and average power at the sample position, tak- 
ing into consideration the differing repetition rates of each laser beam after passing through their respective mechanical choppers. The compressed Raman pump has a bandwidth of $\sim 39 \mathrm{~cm}^{-1}$, measured using a fiber-coupled spectrograph (Brolight BIM6602-02). In configuration 2, fluences of $\sim 170 \mu \mathrm{J} / \mathrm{cm}^{2}$ and $\sim 280 \mu \mathrm{J} / \mathrm{cm}^{2}$ are used for the $267 \mathrm{~nm}$ excitation pump, and $400 \mathrm{~nm}$ Raman pump respectively. The compressed Raman pump has a bandwidth of $\sim 20 \mathrm{~cm}^{-1}$. Additional experiments using a $400 \mathrm{~nm}$ excitation pump also create excited state populations in both DHI and DHICA (measured using TA), however this is likely due to two-photon absorption, as the materials show close to $0 O D$ absorption at this wavelength and so these experimental results are not included here.

In both configurations, 300, 000 shots are collected for 28 time points on a $-3 \mathrm{ps}$ to $1 \mathrm{~ns}$ time range, on a linear-log time scale, and post-processed as discussed in section 4.4.2.

\subsubsection{Spectrometer configurations}

Limitations in achieving suitable spectral resolution for the IR spectrometer over the full vibrational manifold means that only excited state data for the spectral range of interest in eumelanin, that is: $1200 \mathrm{~cm}^{-1}-1800 \mathrm{~cm}^{-1}$, are collected during the experiment. The transient absorption and ground state Raman spectra are collected across a wider spectral range of $200 \mathrm{~cm}^{-1}-2000 \mathrm{~cm}^{-1}$ as shown in figure 5.5, but the FSRS surfaces are analysed only across the region of interest for eumelanin, $1200 \mathrm{~cm}^{-1}-1800 \mathrm{~cm}^{-1}$, as shown in figure 5.7 .

\subsection{Experimental results}

\subsubsection{Optical properties}

\subsubsection{Absorption}

The UV-Vis absorption spectrum of DHI and DHICA monomers prepared in configuration 1 as discussed in section 5.3.1.1 are shown in figure 5.3. A strong peak in the DHICA spectrum can be seen at around $315 \mathrm{~nm}$, in good agreement with literature ${ }^{45]}$, while DHI exhibits the same approximate peak position but at a much lower $O D$ in comparison, assumably due to its lower sample concentration during these experiments ${ }^{[143]}$. It should be noted the UV-Vis taken prior and post experiments in panel 
$b$ (solid and dashed curves) show some absorbance degradation, particularly that of DHI, however the high shot numbers and repeated time-delay runs used in the FSRS experiment should effectively average out any excited state artefacts caused by this.

\subsubsection{Electronic properties: transient absorption}

The TA spectra of materials is collected during FSRS experiments as a result of the shot sequencing described in 4.4 .2 of chapter $\mathrm{A}$. The spectral range of interest in eumelanin's vibrational signatures for this project (and hence also for DHI and DHICA) spans the $1200 \mathrm{~cm}^{-1}$ to $1800 \mathrm{~cm}^{-1}$ band. Because of this, only a limited spectral range for TA (which would normally span UV - IR) is collected during these FSRS experiments. A separate TA experiment across a wider spectral range, conducted by Aleksandra Ilina, using the same excitation pump powers and sample preparation as configuration 1 (but with no Raman pump) is presented in figure 5.4. Panels (a) and (b) show the TA spectral traces for DHI and DHICA using $267 \mathrm{~nm}$ UV excitation.

It can be seen that the peak of the PIA bands for each material are at $\sim 1.7 \mathrm{eV}(730 \mathrm{~nm})$ for DHI, and $\sim 1.3 \mathrm{eV}(886 \mathrm{~nm})$ for DHICA. There is some ambiguity however, due to scattering artefacts caused by the $800 \mathrm{~nm}$ fundamental laser pulse - these regions are shown on the figure as transparent yellow blocks. This aside, it seems that the DHICA PIA peak band is consistent with that seen in eumelanin's TA spectra - discussed further in section 6.2 of chapter 6- while the DHI PIA band peak is at a higher energy. This could also explain the magnitude stronger FSRS signal captured for DHICA compared with that of DHI - as the PIA band peak for DHICA is closer to resonance with the 800 $n m$ Raman pump than for DHI.

Kinetic traces in panels $c$ and $d$ show the rapid rise of both PIA bands, averaged around $1.9 \mathrm{eV}$. Decay times are on $p s$ to $n s$ time scales, and interestingly, DHICA's excited state decays more rapidly than that of DHI. These TA spectra and kinetics provide a benchmark to learn whether FSRS dynamics occur within the excited state lifetime. It should be noted, that due to the high pump powers required to maintain strong SNR, and rapid polymerisation of these materials in air, it is likely that some oligomerisation has occurred. This means that each sample may also contain dimer and trimer (and higher order) oligomers, as opposed to being purely monomeric. Examples of DHI and DHICA structures formed under such polymerisation are presented in chapter 1 . 
transient absorption of eumelanin's sub-units

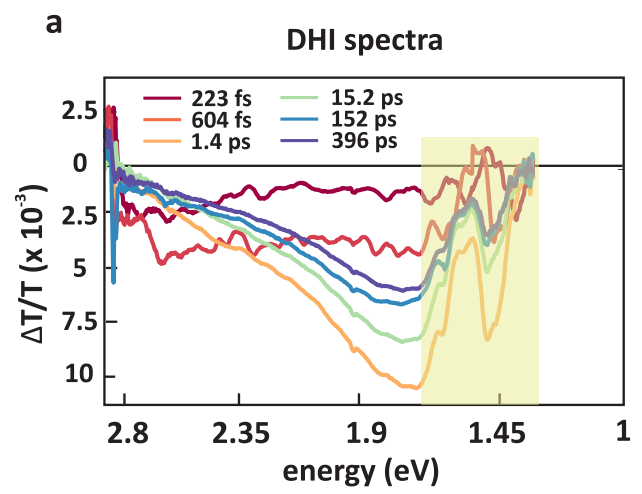

b

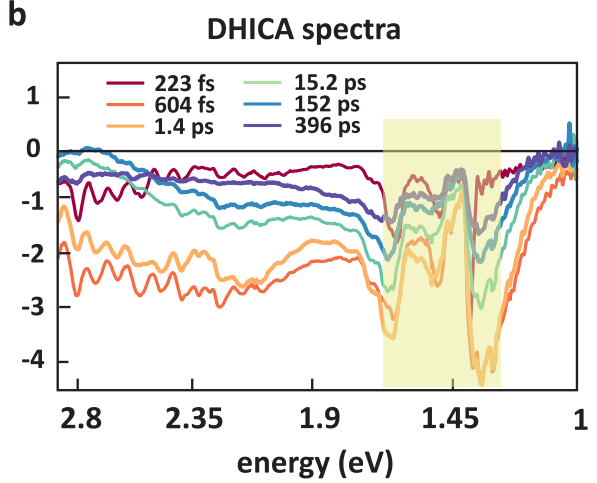

c

d
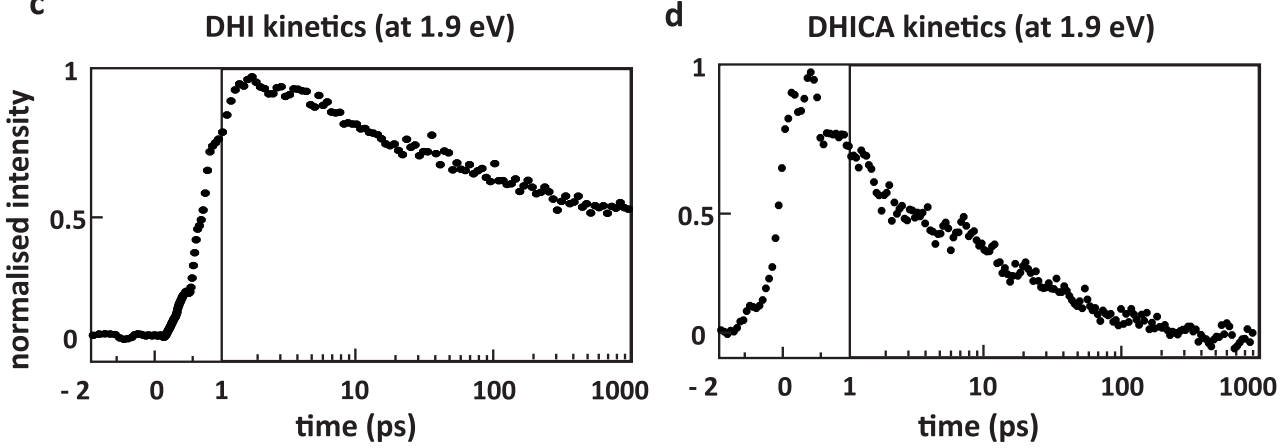

Figure 5.4: Normalised TA spectra for DHI and DHICA dissolved in de-gassed water are shown in panels $a$ and $b$. Due to polymerisation both in air and upon light excitation, it is likely that these species are present in their oligomer forms, rather than purely monomeric. Positive peaks shown in the $1.4-1.6 \mathrm{eV}$ regions below the zero line (highlighted as yellow rectangles) are artefacts due to scatter from the $800 \mathrm{~nm}$ fundamental during conversion of the fundamental pulse to the supercontinuum probe. Decay kinetics averaged around $1.9 \mathrm{eV}(730 \mathrm{~nm})$ are shown in panels $c$ and $d$. The TA spectra and kinetics presented here were collected by Aleksandra Ilina, of the Victoria University of Wellington.

\subsubsection{Ground state Raman}

\subsubsection{Ground state Raman spectra of DHI and DHICA}

The ground state Raman modes of the DHI and DHICA monomers have been previously documented ${ }^{[149]}$. Figure 5.5 collected using an $800 \mathrm{~nm}$ Raman pump shows good agreement with literature for DHICA. There are seven main peaks of interest: at the higher vibrational frequencies, the $\mathrm{C}=\mathrm{O}$ stretching mode of the carboxylic acid 
ground state Raman of eumelanin's sub-units (water solvent)

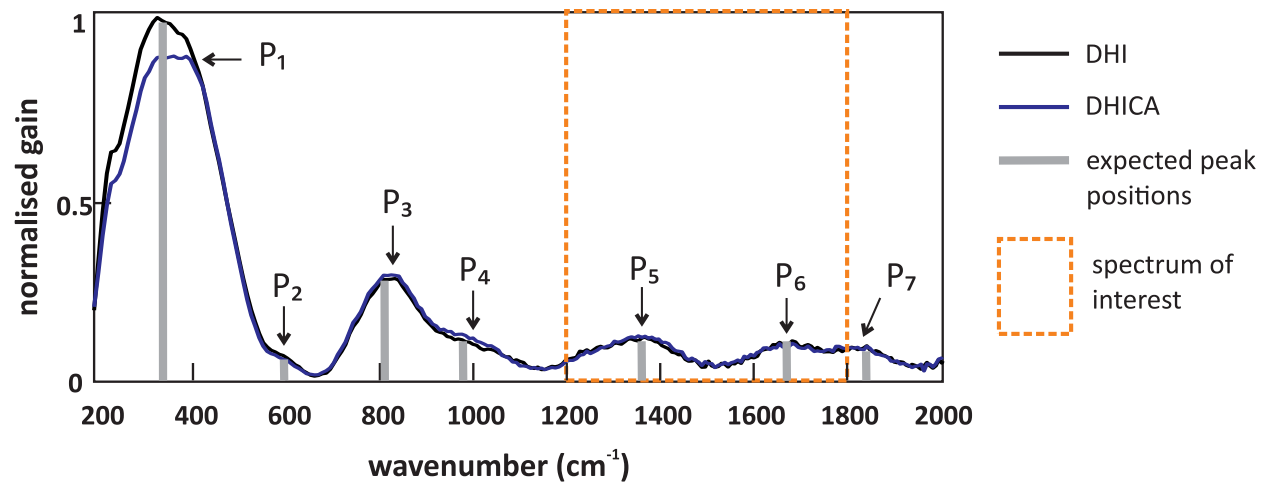

Figure 5.5: The ground state Raman spectrum collected for DHI and DHICA are presented as the black and blue curves, respectively. Expected peak positions are shown as grey lines. There are 7 distinct peaks ranging across the visible and IR regions over a $200-2000 \mathrm{~cm}^{-1}$ spectrum. The orange dashed box indicates the spectral range of interest, $1200-1800 \mathrm{~cm}^{-1}$, for the comparison an reference of DHI and DHICA with eumelanin's key Raman peaks. This spectral range is focused on in this chapter.

group present at $\sim 1800 \mathrm{~cm}^{-1}$ and six lower frequency modes between $400 \mathrm{~cm}^{-1}$ and $1630 \mathrm{~cm}^{-1}$. These are assigned to in-plane and out-of-plane $\mathrm{C}-\mathrm{H}$ bending modes, and deformation modes of the pyrrole and benzene rings. These main 7 peaks collected in this experiment are presented in table 5.2 .

Of interest is the strong overlap of the ground state Raman peaks for both the DHI and DHICA subunits, which is unexpected at higher frequencies for DHI, due to the unique presence of the carboxylic acid group in DHICA, producing peaks distinct from the DHI subunit around $1700-1800 \mathrm{~cm}^{-1}$. It is possible that any broadening effects, particularly of the DHI peak assigned via DFT at $\sim 1739 \mathrm{~cm}^{-1}$, that could be resolved have already been consumed in the resolution limit of the spectrometer, and could explain the apparent presence of the $1753 \mathrm{~cm}^{-1}$ band in the DHI spectrum, which should only present in the DHICA material. However it is more likely that polymerisation of the unstable DHI monomer has occurred, contributing to peak broadening, and an overlap with the expected $\mathrm{C}=\mathrm{O}$ stretching modes in DHICA. Literature shows similar spectral positions for the ground state Raman spectra of DHI and DHICA materials, with the key difference noted as being an overall broadening of the DHICA spectra compared to DHI at wavenumbers below $1,100 \mathrm{~cm}^{-1}$ and above $1,700 \mathrm{~cm}^{-1}$. 
Experimental Raman peak assignments for DHI and DHICA in the ground state

\begin{tabular}{|l|l|l|}
\hline \hline peak number & Stokes shift $\left(\mathrm{cm}^{-1}\right)$ & mode assignment \\
\hline$P_{1}$ & 377 & C-H in-plane bending \\
\hline$P_{2}$ & 621 & C-H in-plane bending \\
\hline$P_{3}$ & 850 & O-H and C-H out-of-plane deformation \\
\hline$P_{4}$ & 1030 & C-H in-plane deformation \\
\hline$P_{5}$ & 1370 & C-N stretching \\
\hline$P_{6}$ & 1688 & C=O stretch of carboxylic acid group \\
\hline$P_{7}$ & 1830 & C=O bend of carboxylic acid group \\
\hline
\end{tabular}

Table 5.2: Peak assignment for experimental ground state Raman; the DHI and DHICA materials are included in one table as they show essentially the same peak positions.

\subsubsection{Mode assignment of the ground state using DFT analysis}

As previously introduced in section 4.5.5.2 of chapter 4 . DFT is a computational method used to model the nuclear structure of molecules, defining motions in which the positions of the nuclei change with respect to each other, i.e their vibrations. The DFT computation provides the frequencies of the calculated vibrations as well as visual graphics of the molecular vibration occurring. We can use this information to form a picture of the actual vibrational mechanism occurring in the molecule mapped from the frequency peaks observed in the Raman spectra.

\subsubsection{Time-resolved excited state Raman}

\subsubsection{FSRS of DHI and DHICA in experimental configuration 1}

The excited state FSRS surfaces for DHI and DHICA in water are shown in the top panels $a$ and $b$ of figure 5.7. In the surfaces we can clearly see evolution of vibrational modes rising and decaying on $\sim p s-n s$ time scales. The surfaces, while visually similar, have slightly varying peak frequencies. Panels $c$ and $d$ of the same figure show the two main spectral components obtained from SVD analysis. The lower panels $e$ and $f$ shows the kinetic components of these spectra. SVD analysis is useful in this instance 


\section{ground state DFT mode assignments}

DHI

\section{DHICA}

$1257 \mathrm{~cm}^{-1}$

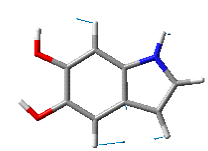

$1383 \mathrm{~cm}^{-1}$

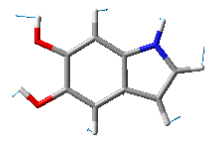

$1423 \mathrm{~cm}^{-1}$

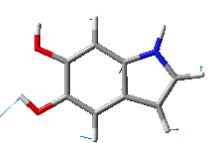

$1588 \mathrm{~cm}^{-1}$

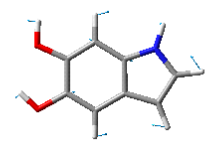

$1675 \mathrm{~cm}^{-1}$

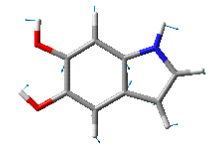

$1729 \mathrm{~cm}^{-1}$

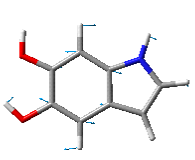

$1265 \mathrm{~cm}^{-1}$

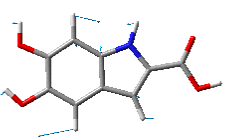

$1342 \mathrm{~cm}^{-1}$

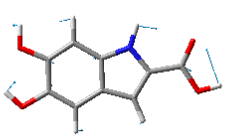

$1432 \mathrm{~cm}^{-1}$

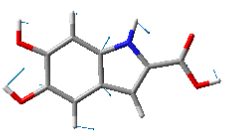

$1574 \mathrm{~cm}^{-1}$

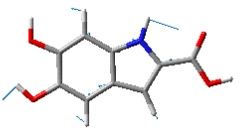

$1613 \mathrm{~cm}^{-1}$

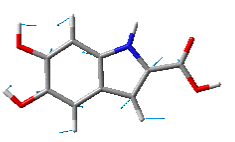

$1731 \mathrm{~cm}^{-1}$

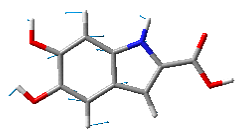

Figure 5.6: The calculated ground state vibrational energies and mode assignments for DHI (panel $a$ ) and DHICA (panel $b$ ) using density functional theory (DFT) analysis are presented. Similarities can be seen between each molecule in case of the breathing modes of the pyrrole 5 and benzene 6 rings. Distinctions at the higher energies are apparent for DHICA where the $\mathrm{C}=\mathrm{O}$ stretching mode in the carboxylic acid group presents. This DFT analysis was performed by Paul Hume of the Victoria University of Wellington using Gaussian 09, Revision D.01 software.

to obtain the time scales of spectral dynamics, which are within the excited state lifetime of the TA signal (shown as the pink dashed curves in panels $e$ and $f$ ). The spectral components extracted by the SVD analysis can't necessarily be interpreted in any useful physical way, as, for example, a shift from one spectral component to another will present the same mathematically as the rise and decay of two distinct species. It is still useful, however, to get an indication of the number of spectral components that are present in the surfaces, in addition to their decay kinetics. 

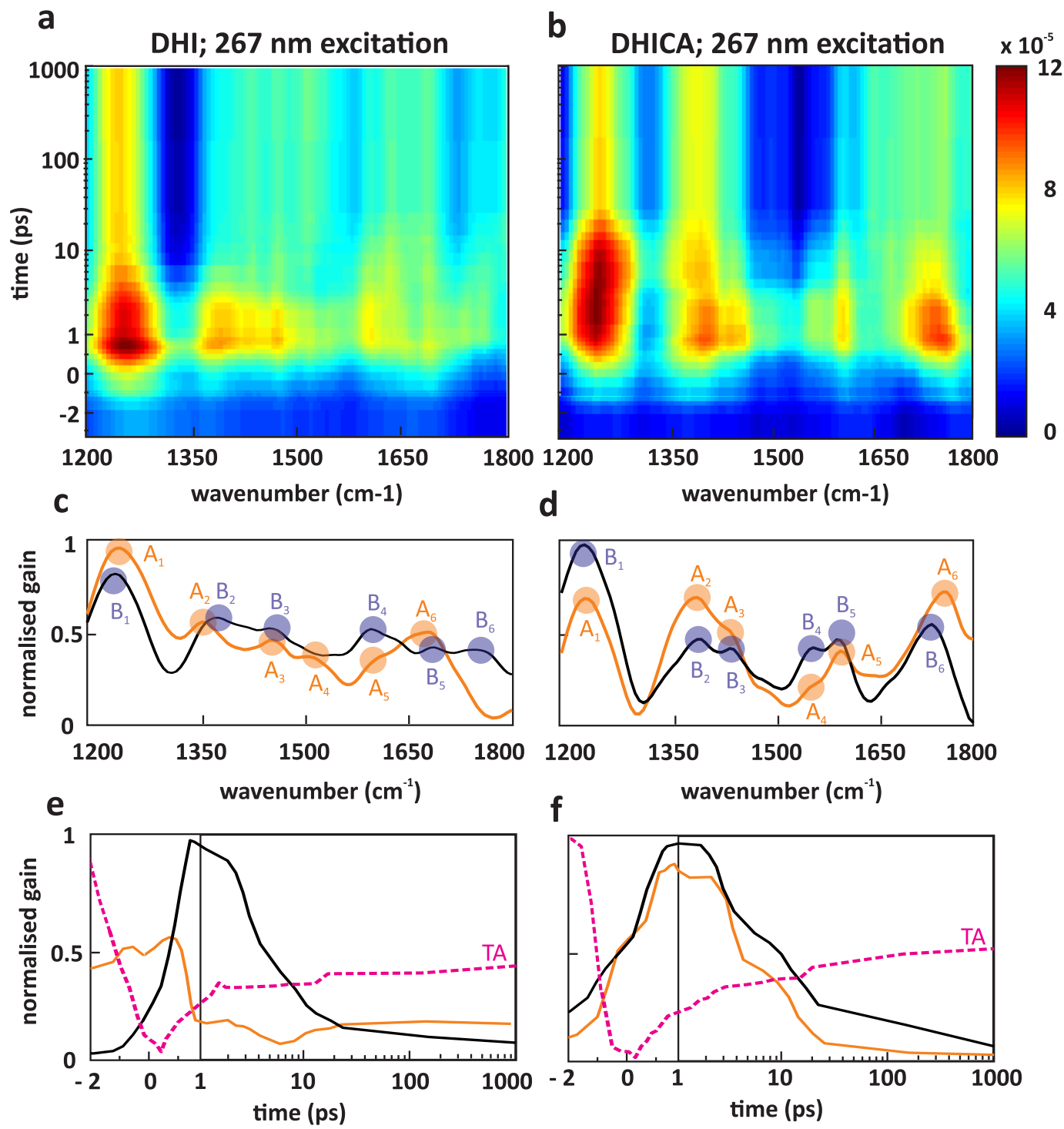

Figure 5.7: Panels $a$ and $b$ show the FSRS surfaces for DHI and DHICA. The rise and decay of the peaks vary slightly in energy and time. SVD analysis for each surface, shown in panels $c$ and $d$, reveal two main spectral components with similar peak positions (highlighted by the orange circles denoted as $A_{n}$ and the blue circles denoted as $B_{n}$ ) for the components within each material. Kinetics traces of these spectral components are shown in panels $e$ and $f$ as orange and black curves, revealing similar rise and decay kinetics between the two materials. The averaged TA data collected at the same time as the FSRS experiments are shown as pink dashed curves, indicating a steep rise in the PIA band with a decay time on the order of 1 ns. 
Further analysis of the raw data surfaces outside of the SVD method is conducted to determine the spectral evolution of the excited state frequencies in time, as well as the their intensity kinetics. Figure 5.8 shows the FSRS surface spectra for each monomer for 5 time points from -1 ps to $4.8 \mathrm{ps}$ as pink - purple curves. The grey bars in these figures represent the central vibrational frequencies of 6 "main peaks" located at 1260 $\mathrm{cm}^{-1}, 1415 \mathrm{~cm}^{-1}, 1460 \mathrm{~cm}^{-1}, 1550 \mathrm{~cm}^{-1}, 1660 \mathrm{~cm}^{-1}$ and $1690 \mathrm{~cm}^{-1}$. These "main peaks" are chosen visually from the raw data FSRS spectral slices, with the support of the mathematically derived SVD spectral analysis. The peak locations are similar between each material, with the higher frequency modes being more distinct in the DHICA molecule. The highest energy mode around $1750 \mathrm{~cm}^{-1}$ is assigned to $\mathrm{C}=\mathrm{O}$ stretching of the carboxylic acid group, present only in DHICA, so a distinct vibrational peak at this frequency for DHICA is consistent with the structure of the material.

By taking a $\pm 50 \mathrm{~cm}^{-1}$ spectral window across each peak location, and using a polynomial fit to obtain the maxima, the peak intensities can be mapped as a function of time. This is shown in panels $a$ and $b$ of figure 5.9 as the coloured curves, with the TA PIA band lifetime overlayed as the dashed black line. Below each plot are the vibrational mode assignments obtained from the ground state DFT analysis presented in figure 5.6. It should be noted, that the ground state and excited state modes will differ from each other, however at the time of this data analysis, only the ground state DFT mode assignments were available. The kinetics show similar rise times for each peak in both materials on a $<p s$ time scale, being at maximum intensity around $1-2$ ps. Interestingly, the decay of each peak in both DHI and DHICA show varying kinetics, indicating the rapid decay of some peaks and a slower decay of others. For DHI, the lower energy modes between $1175 \mathrm{~cm}^{-1}$ and $1534 \mathrm{~cm}^{-1}$ decay faster than the higher energy modes above $1630 \mathrm{~cm}^{-1}$, and show a typically exponential function. A similar trend is seen in DHICA, with the exception of the mode at $\sim 1240 \mathrm{~cm}^{-1}$ which indicates a longer lived excited state vibrational mode at this frequency.

\subsubsection{Dynamic comparison for different solvents}

A further comparison is made between DHICA dissolved in water, and DHICA dissolved in ethanol in a separate experiment, with the ethanol dilution prepared in the same method as for the water dilution and in the experimental parameters of configuration 1 discussed in section 5.3.1.1. Panel $c$ of figure 5.10 shows the spectral peaks of DHICA dissolved in ethanol as less distinct than that of a water solution. These 
a

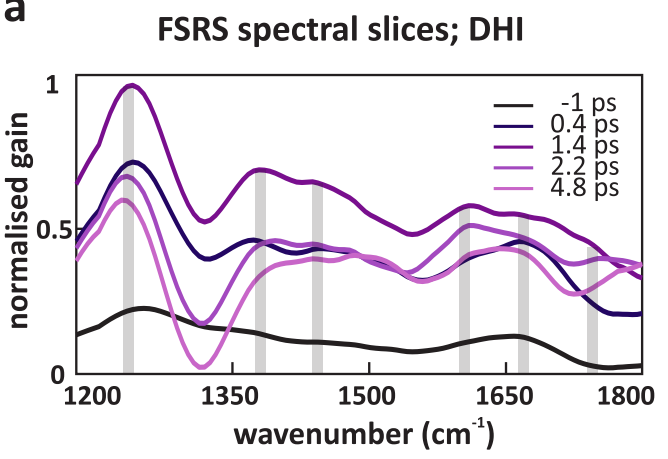

b FSRS spectral slices; DHICA

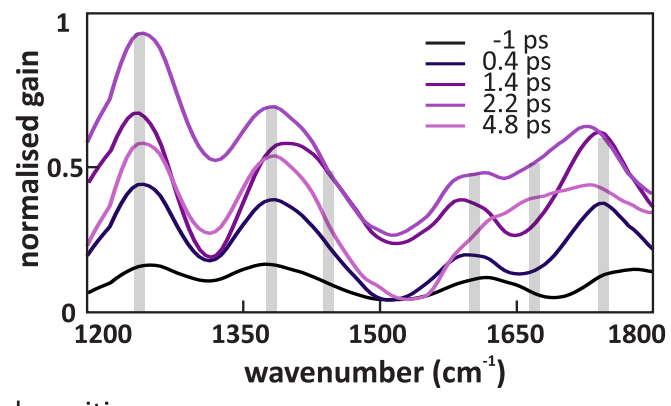

Figure 5.8: Spectral traces for each monomer are shown in panel $a$ for DHI and panel $b$ for DHICA. The traces were taken across the FSRS surface at 5 time points. The rise of each vibrational mode from pre time-zero (black curve) can be seen, with the lower energy peak at around $1240 \mathrm{~cm}^{-1}$ dominating the higher energy peaks. An apparent blue-shift is also observed for this peak upon decay. The higher energy modes show a similar rise and decay, with the exception of the central energy modes around $1500 \mathrm{~cm}^{-1}$ which appear to rise and decay more rapidly for each material. The 6 main peak locations, used to take kinetic traces in the next figure, figure 5.9, are represented in this figure as grey lines.

"smeared" peaks may indicate a more straight forward vibrational dissipation mechanism within the material which is unable to structurally interact with the solvent. It should be noted that any such interaction would occur regardless on a much slower time scale than the dynamics observed here, due to the large $\mathrm{CH}_{2} \mathrm{CH}_{3}$ group in ethanol limiting bonding interactions.

The kinetics of these peaks are taken from the spectral slices shown in panel $a$ and $c$, with a comparison shown in panels $b$ and $d$ for the sample dissolved in water and in ethanol respectively. Comparison of these dynamics may give interesting insights into structural rearrangement of the molecule as being solvent dependent. Here, it is noted when comparing decay kinetics between DHICA in water (panel $b$ ) and DHICA in ethanol (panel $d$ ) that the decay kinetics for each peak of DHICA dispersed in ethanol are almost identical for each mode, on a $p s$ time scale. When compared to DHICA dispersed in water, the peak kinetics for the $1240 \mathrm{~cm}^{-1}$ mode are also matched between the two samples, while the other peaks show varying decay rates. This indicates that there may be a structural change to the DHICA molecule in the water solvent that doesn't 

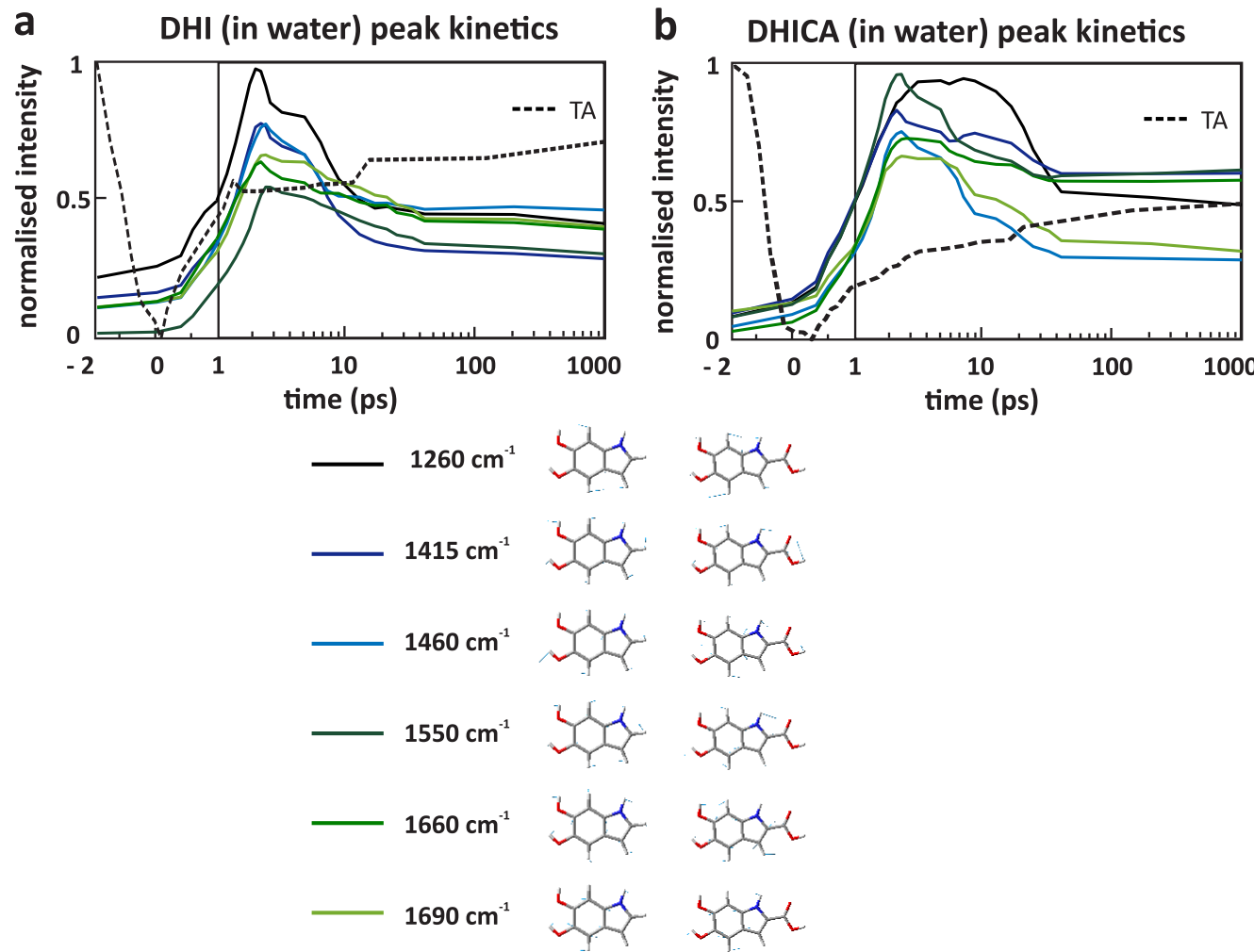

Figure 5.9: Panels $a$ and $b$ show the kinetic traces integrated across the 6 key vibrational peak positions for DHI and DHICA in water identified from the FSRS surfaces. The black curve is centred at $1240 \mathrm{~cm}^{-1}$, with the other 5 curves being centred at $1410 \mathrm{~cm}^{-1}$ (dark blue curve), $1507 \mathrm{~cm}^{-1}$ (light blue curve), $1680 \mathrm{~cm}^{-1}$ (light green curve), $1767 \mathrm{~cm}^{-1}$ (dark green curve) and $1842 \mathrm{~cm}^{-1}$ (darkest green curve). The rise of each peak occurs on a $p s$ time scales for both materials, while the decay rates show slightly differing trends. Within each material the lower energy bands between 1175 and $1534 \mathrm{~cm}^{-1}$ show a similar, rapid exponential decay with the exception of the peak centred at $1240 \mathrm{~cm}^{-1}$ (black curve) which has a longer lifetime in the DHICA molecule. The transient absorption for each material is shown as the black dashed curves.

occur in ethanol, perhaps in support of ESPT as an excited state dissipation mechanism for DHICA.

It is apparent that these initial results demonstrate the rapid structural change of DHICA as being solvent dependent. This supports the hypothesis of ESPT between DHICA and water - a mechanism restricted for DHICA when dispersed in ethanol. Investiga- 
Comparison of solvent in DHICA kinetics

a DHICA (in water) peak spectra

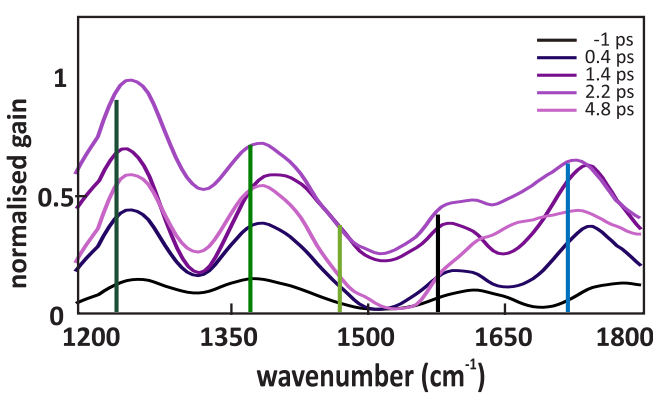

C DHICA (in ethanol) peak spectra

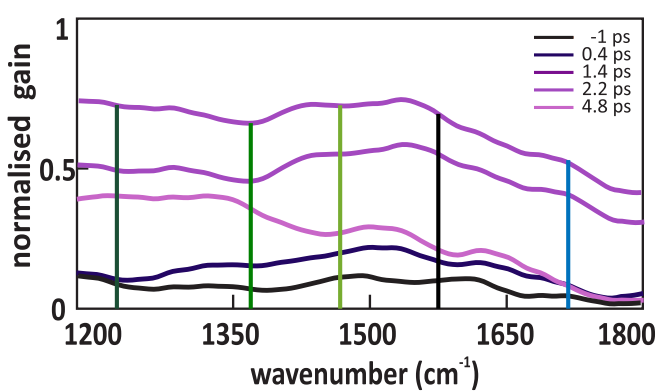

b DHICA (in water) peak kinetics

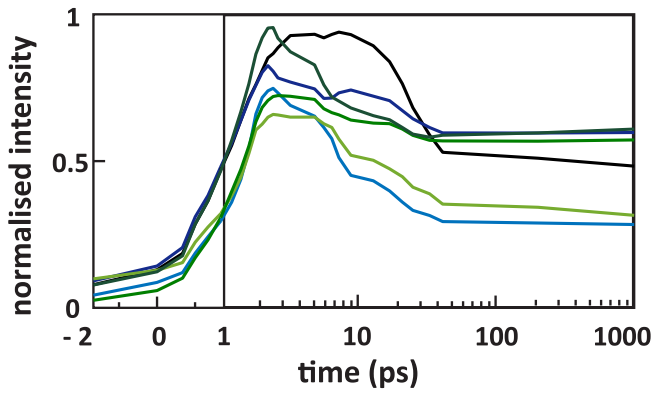

d DHICA (in ethanol) peak kinetics

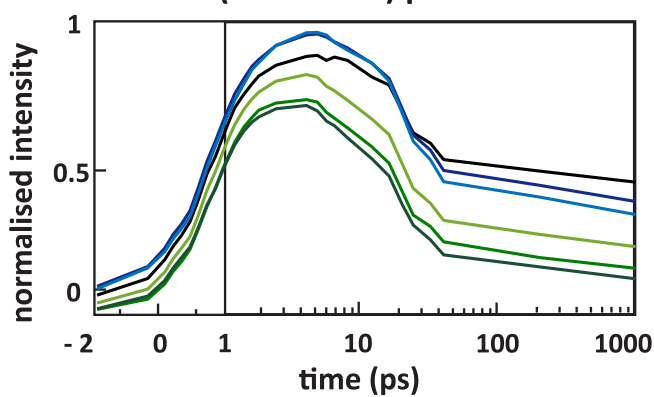

Figure 5.10: Panels $a$ and $b$ show the spectral and kinetic traces for DHICA in water, with the spectral locations of each kinetic trace curve colour coded. Panels $c$ and $d$ show the same for DHICA in ethanol.

tion into the solvent dependence of DHICA's excited state decay mechanism, and the proposed model for ESPT, is the focus of the following section.

\subsubsection{Evidence of excited state proton transfer (ESPT)}

As previously shown in the schematic of figure 5.2 and outlined in section 5.2 of this chapter, ESPT can occur for DHICA dissolved in neutral water. Upon dilution, DHICA will assume a deprotonated carboxylate anion via inter-molecular proton transfer of a proton in the $\mathrm{COOH}$ group to the solvent bath. Following photo-excitation, the phenol groups can transfer protons to the solvent via ESPT, forming an excited state phenolate di-anion. The excited state di-anion then relaxes via fluorescence on a $n s$ timescale allowing the molecule to then relax back to its ground state via subsequent fluorescence and proton scavenging. The possibility of ESPT as an energy decay mechanism is the first hypothesis put forward in this research project. 

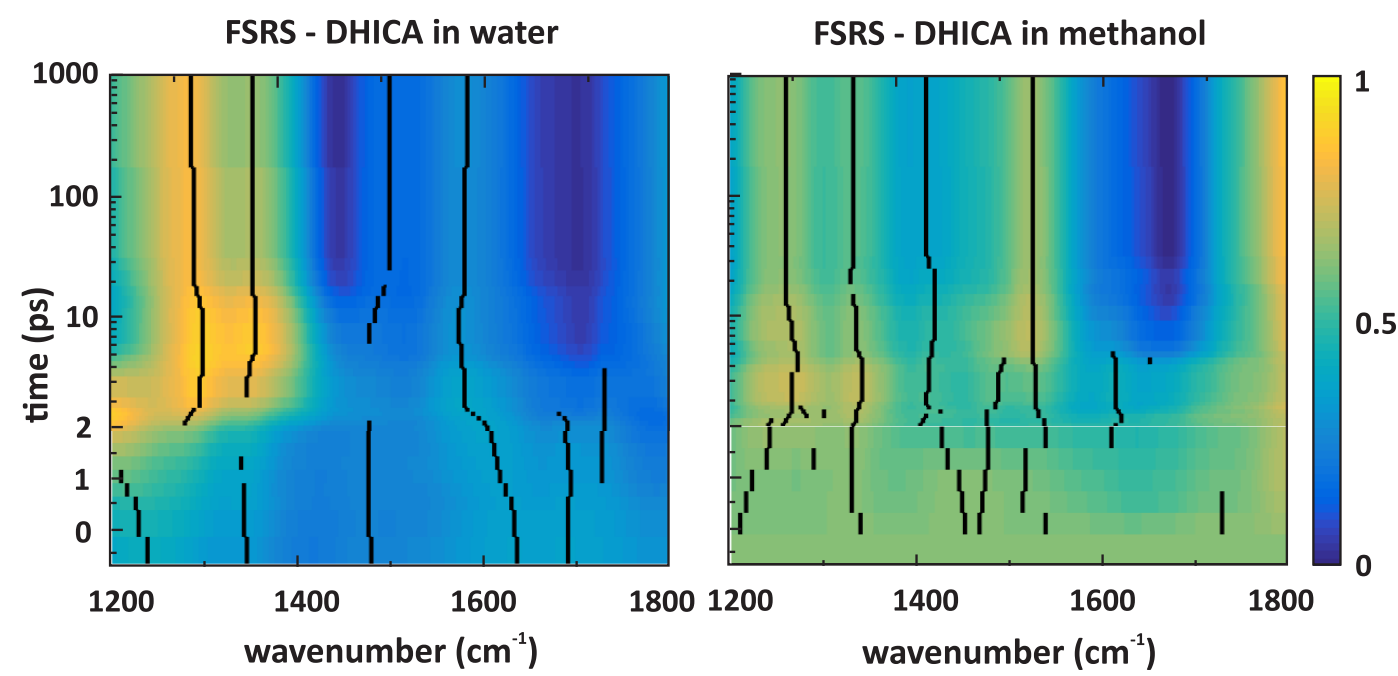

Figure 5.11: FSRS surfaces of DHICA in water and methanol. The differential of each spectral slice in time was taken of the surfaces to identify spectral peaks, shown as the black curves overlaying each surface. Immediately apparent is the peak shifting in panel $a$, especially around $1600 \mathrm{~cm}^{-1}$, which is not present in the methanol preparation.

Hypothesis: Excited state proton transfer is a primary deactivation pathway for DHICA under photo-excitation when dissolved in a $\mathrm{pH}$ neutral solvent.

To test this hypothesis, a comparison of the experimental DHICA FSRS data for two solvents is compared with DFT analysis of the ESPT process. Samples of DHICA are prepared according to configuration 2 with details given in section 5.3.1.1, for both water and methanol solvents. The excited state FSRS surfaces for DHICA in water and in methanol are shown in figure 5.11 .

A differential analysis was performed on each surface for each of the spectral time slices. This analysis gives the peaks and troughs of the spectral surface as crossing points across a zero access, that is, the derivative value $=0$ when the surface is at a peak or trough. A zero crossing going from positive to negative differential values indicates a peak in the original surface, while the opposite indicates a trough. Matlab code given in appendix C was written to identify the peak values only, and overlay these as a mesh plot on the FSRS surface. These peak locations are shown as the black 


\section{DFT analysis of DHICA proton transfer states}
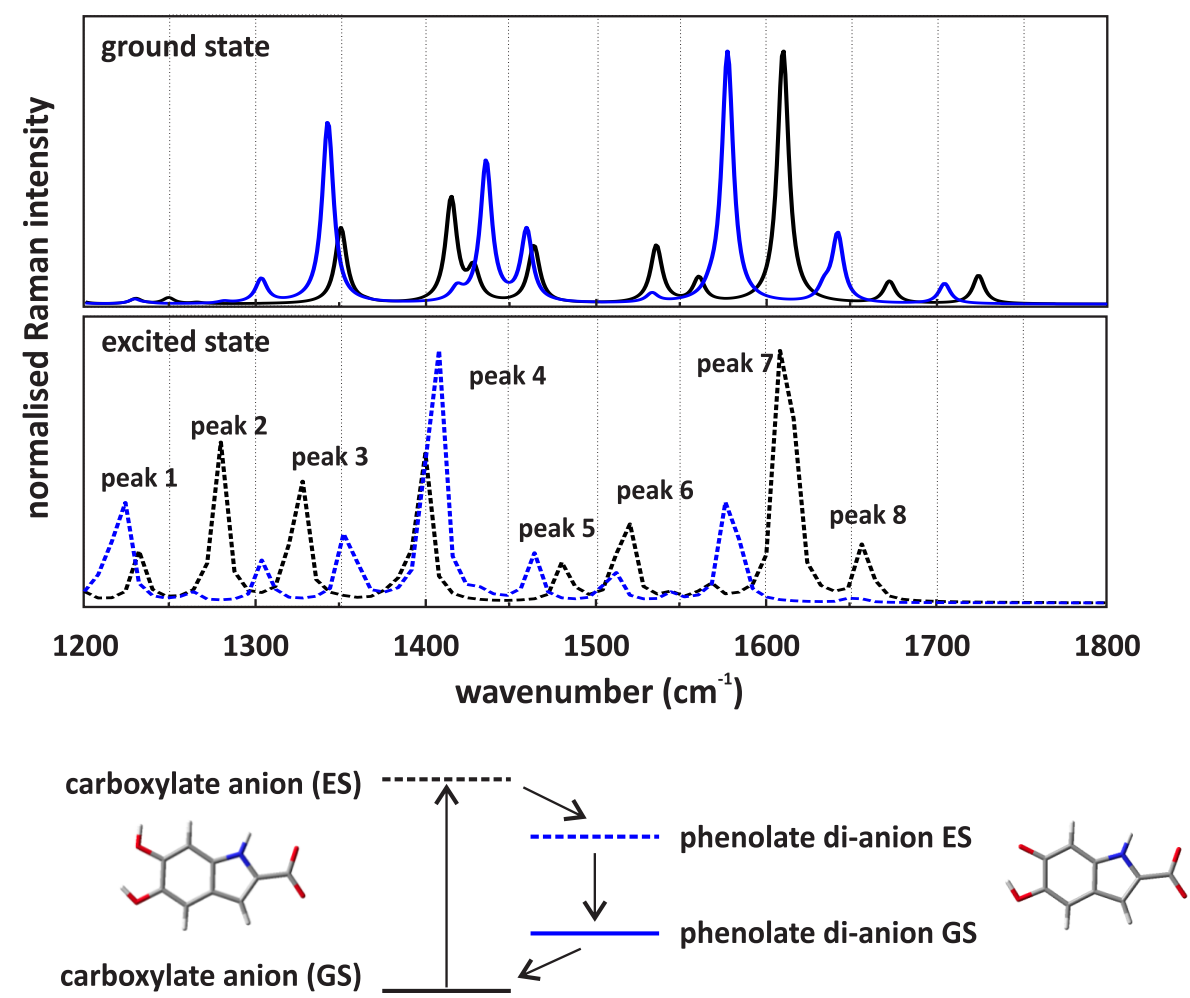

Figure 5.12: DFT analysis performed for the DHICA carboxylated anion and phenolate di-anion are presented. The two molecular structures are shown under the graph, with the carboxylate anion having lost an $\mathrm{H}+$ from the $\mathrm{COOH}$ group (via inter-molecular proton transfer to the solvent bath) and the phenolate di-anion having lost an additional $\mathrm{H}+$ to the solvent from the $\mathrm{OH}$ phenol site. Identified peaks in the DFT analysis are shown on the graph as peak 1 - peak 8 , with the black curve being the carboxylate anion and the blue curve being the di-anion. Clear spectral shifts can be seen in each of the peak locations, indicative of a change in the vibrational energy frequencies and modes between the two materials.

lines overlayed on each FSRS surface in figure 5.11 .

To compare experimental data with the DFT analysis, DFT calculations were on the carboxylated DHICA anion and the phenolate di-anion in both their ground and excited states. The peaks were calculated using the ground and excited DFT methodologies described in section 4.5 .5 .2 of chapter 4 over a $1200 \mathrm{~cm}^{-1}-1800 \mathrm{~cm}^{-1}$ spectral range. The graphs of the ground state and excited state peaks calculated in the analysis are presented in figure 5.12 . 
experimental peak shifts compared to DFT analysis of the excited state

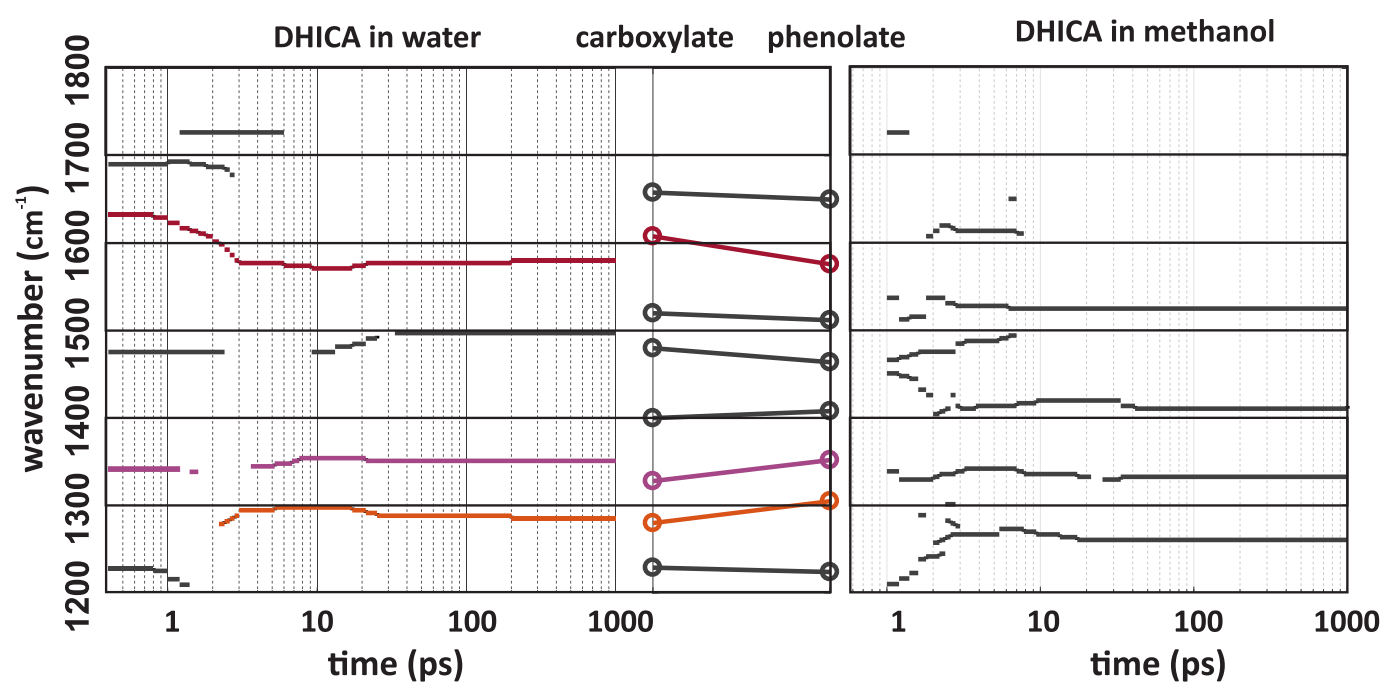

Figure 5.13: Comparison of peak frequency shifts of DHICA dissolved in 2 solvents - water and methanol - with DFT analysis of the excited state ESPT process from carboxylate to phenolate shown as a shift in frequencies in the middle of each experimental figure. The colour codes of the left hand plot match modes of interest with the DFT analysis peak shifts. Of note is the absence of clear peak shifts in the methanol preparation, where ESPT is prohibited.

This figure shows the ground state peaks as blue and black curves for the carboxylate and phenolate molecules respectively. In the excited state graph (dashed curves) 8 peak pairs are identified. The frequencies of each of these peak pairs was taken from the plot and used to compare the shifting of frequencies between the excited states where ESPT occurs. The peak values are then used in comparison with those peak frequencies identified in the differential analysis. The carboxylate and phenolate ground state vibrational modes assignments calculated using DFT analysis are presented in figure D.1 of appendix D

Figure 5.13 shows the experimental peak locations as a function of time. In the central panel of this figure, the 8 DFT peaks of the carboxylate and phenolate excited states are represented. The left hand panel shows the experimental data of DHICA dissolved in water (where ESPT can occur), while the right hand panel shows DHICA in methanol (where ESPT cannot occur). Comparison can be made here between the experimental data and the DFT analysis. For the case of the water solvent, the red, purple and or- 
ESPT model for DHICA in water

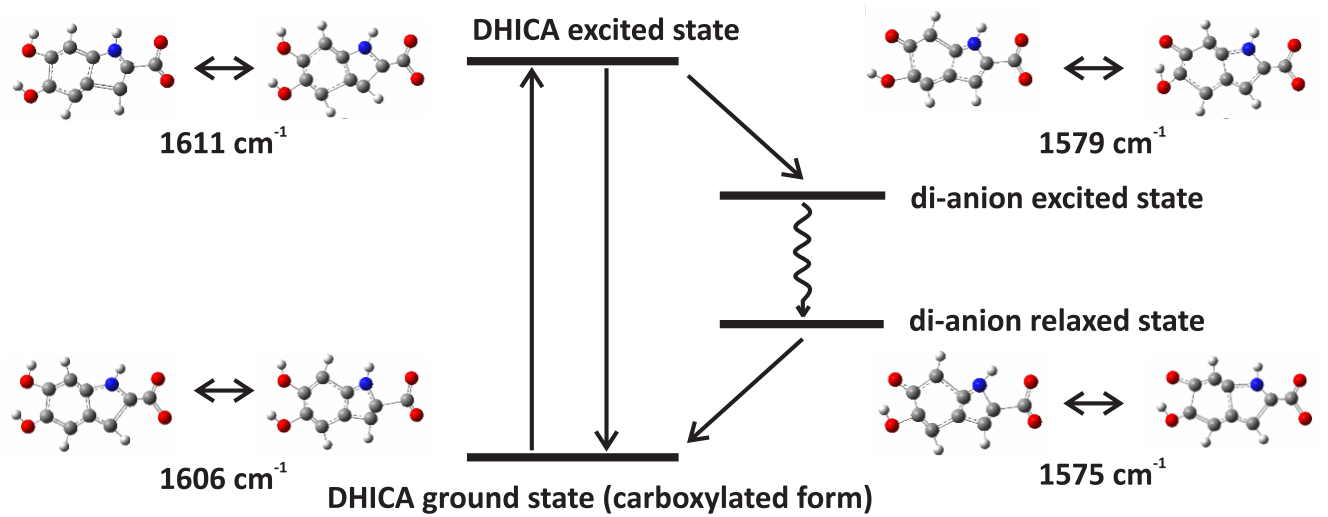

Figure 5.14: Graphical representation of the DFT vibration modes around 1600 $\mathrm{cm}-1$ in the ESPT model. Here we can observe that the vibrations of the molecules are similar in the deformation of the pyrolle and benzene rings, with the absence of the wagging of the hydrogen in the phenol group upon de-protonation.

ange curves are colour matched to similar peak shifts calculated in the DFT analysis. In the case of the methanol solvent, it is clear that the surface are less comparable to the DFT peak shifts. Of particular interest is the vibrational mode with a frequency around $1600 \mathrm{~cm}^{-1}$ where, in water, a strong shift from higher to lower frequencies is apparent in both the water preparation and in the dFT analysis. However this mode does not appear strongly in the methanol preparation.

Figure 5.14 shows the DFT calculated peaks for the ESPT process around the $1600 \mathrm{~cm}^{-1}$ frequency. The vibrational modes are shown graphically for each of the ESPT states. In each case the dominant vibration is the deformation of the pyrolle and benzene rings, with the absence of the $\mathrm{H}$ wagging mode in the phenol group in the phenolate vibrations.

We believe that the correlation observed in the experimental FSRS data compared to the peak shifts calculated in the DFT analysis indicates strong evidence of ESPT occurring in the DHICA monomer dissolved in water, while no such correlation is seen in the methanol preparation. Using this spectroscopic signature of the DHICA monomer, we next look to the eumelanin macro-molecule in chapter 6 to see if similar peak shifts can be observed. 


\subsection{Chapter summary}

This chapter presents key vibrational references for eumelanin's subunit building blocks, DHI and DHICA. The main vibrational peaks of DHI and DHICA have been identified and assigned using a combination of FSRS experiments and DFT analysis. Their vibrational dynamics are shown to be on $p s$ timescales in comparison with the longer lived electronic excited state species shown in the transient absorption data.

SVD analysis was performed on DHI and DHICA to understand the number of spectral species in each surface as well as the time signatures of their excited state lifetimes. It is demonstrated that DHI and DHICA bear similar ground state and dynamic vibrational features.

Using a visible Raman pump to achieve resonance with the DHICA monomer's PIA band, two preparations of the molecule are compared, one in water where ESPT can occur, and one in methanol where this relaxation pathway is prohibited. Spectral peak shifts are calculated from the FSRS surfaces of these samples and compared to DFT calculations of the ESPT process. In comparison, strong evidence is shown for ESPT as a key relaxation pathway for DHICA dissolved in a neutral $\mathrm{pH}$ solvent.

These results provide spectral signature benchmarks of eumelanin's monomers which are used in the following chapter where eumelanin is studied in films and solutions. 


\section{Chapter 6}

\section{Ultrafast vibrational spectroscopy of eumelanin pigment}

\subsection{Introduction}

FSRS is applied to eumelanin pigment prepared as thin films and dispersion preparations. In this chapter we present FSRS results and analysis of eumelanin, as well as TA measurements conducted for 3 excitation wavelengths. Two hypotheses are tested using the FSRS measurements along with TA and resonance Raman experiments conducted in the ground state. Evidence of excited state proton transfer (ESPT) in eumelanin films is proposed, using its DHICA subunit vibrational decay mechanisms presented in the previous chapter 5 as a reference.

This chapter is set out as follows:

- Eumelanin's ground state Raman features are assigned using a pseudo-Voigt function fitting analysis. A study of eumelanin's ground state absorption and transient absorption spectra is used to determine the optimal Raman pump frequency to achieve resonance with eumelanin's PIA band in order to increase the Raman gain SNR.

- Comparisons between Raman features seen in eumelanin and those of amorphous carbon are discussed, noting the similarities between the two materials in the ground state in terms of ordered and disordered micro-molecular structures.

- The preparation of eumelanin films (and dispersions) used in the FSRS experi- 
ments are presented, along with the experimental configuration of the FSRS system.

- FSRS data is presented for eumelanin films for two excitation wavelengths in the UV $(267 \mathrm{~nm})$ and visible $(400 \mathrm{~nm})$ regions. Proposed models to explain the observed decay kinetics in the FSRS data are next presented as two hypotheses:

1. Excitation's of eumelanin decay via localised pathways

2. ESPT is a primary vibrational decay mechanism in eumelanin

- The potential effects of heating under light excitation on eumelanin's vibrational modes is explored, including results from temperature dependent spontaneous Raman experiments of eumelanin films in the ground state.

- Finally, vibrational data using time-resolved impulsive stimulated Raman spectroscopy (TR-ISRS) performed in collaboration with Riken Laboratories WakoShi, Japan is presented and discussed.

\subsection{Transient absorption of eumelanin films}

Transient absorption spectroscopy (TA) ${ }^{\sqrt{40 \mid 4243}}$, as introduced in chapter 2, is performed on eumelanin films. Panel's $a, b$ and $c$ of figure 6.1 show the TA spectra of eumelanin for three different excitation wavelengths spanning the IR to visible regions at $1.7 \mathrm{eV}$ $(712 \mathrm{~nm}), 2.3 \mathrm{eV}(532 \mathrm{~nm})$ and $3.1 \mathrm{eV}(400 \mathrm{~nm})$. Six spectral time slices taken between $233 \mathrm{ps}$ and $396 \mathrm{ps}$ are shown for each excitation, indicated by the coloured curves. It should be noted that the two different spectral ranges shown in these spectra are due to the use of two different nonlinear crystals to generate the whitelight probe. In panels $a$ and $b$ a YAG crystal is used to observe the visible and IR regions, while in panel $c$ a $\mathrm{CaF}_{2}$ crystal is used to observe the ground state bleach towards the UV region. The spectra demonstrate that, interestingly, the GSB peak location (shown as the light pink bars) for each excitation mirrors that of the excitation pump wavelength, while the PIA bands (shown as the light blue bars) peak at the same $1.3 \mathrm{eV}$ energy (954 $\mathrm{nm}$ ) independent of excitation pump wavelength. This provides evidence that eumelanin's electronic excitations are wavelength dependent, meaning chromophores of differing energies within the material are being individually excited.

As shown in panel $d$ of the same figure, the decay of the GSB and PIA bands occur on rapid, $p s$ time scales. This figure shows the decay kinetics of the spectra shown in 


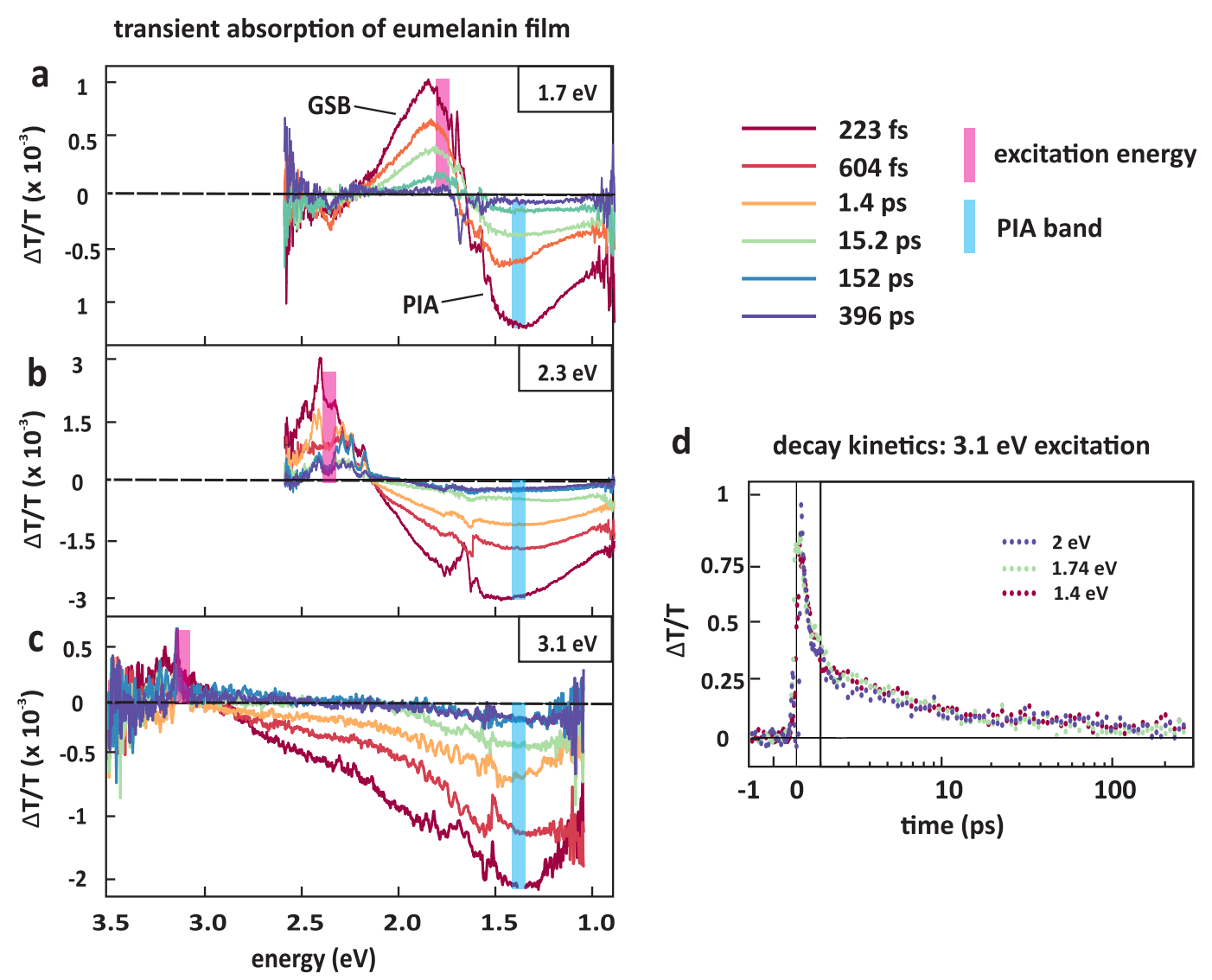

Figure 6.1: Transient absorption spectral traces of eumelanin films are shown in panels $a-c$ for three different pump wavelengths, at $1.7 \mathrm{eV}(712 \mathrm{~nm}), 2.3 \mathrm{eV}$ (532 $\mathrm{nm}$ and $3.1 \mathrm{eV}(400 \mathrm{~nm})$. Panel $b$ shows normalised decay kinetics for the spectra shown in panel $c$ averaged across the three spectral locations at $2 \mathrm{eV}, 1.74 \mathrm{eV}$ and $1.4 \mathrm{eV}$.

panel $c$ (excitation at $3.1 \mathrm{eV}(400 \mathrm{~nm})$ ) for three different spectral locations, across a 1.4 $\mathrm{eV}-2 \mathrm{eV}$ range to capture both the GSB and PIA bands. We can see that the decay kinetics for the GSB and PIA bands are the same.

Another interesting feature within the TA dynamics is a lack of fluence dependence on eumelanin's electronic excited state decay, as shown in figure 6.2. This figure shows, in panel $a$, three spectral traces taken at $233 f$ for three different pump powers, specifically $250 \mathrm{~mW}, 500 \mathrm{~mW}$ and $100 \mathrm{~mW}$. The pump excitation wavelength in these experiments is $532 \mathrm{~nm}$. Using a circular spot size with a $\sim 500 \mu \mathrm{m}$ diameter, these powers correspond to fluences of 85,170 and $340 \mu \mathrm{J} / \mathrm{cm}^{2}$ respectively - fluence is calculated 
transient absorption fluence dependence of eumelanin film
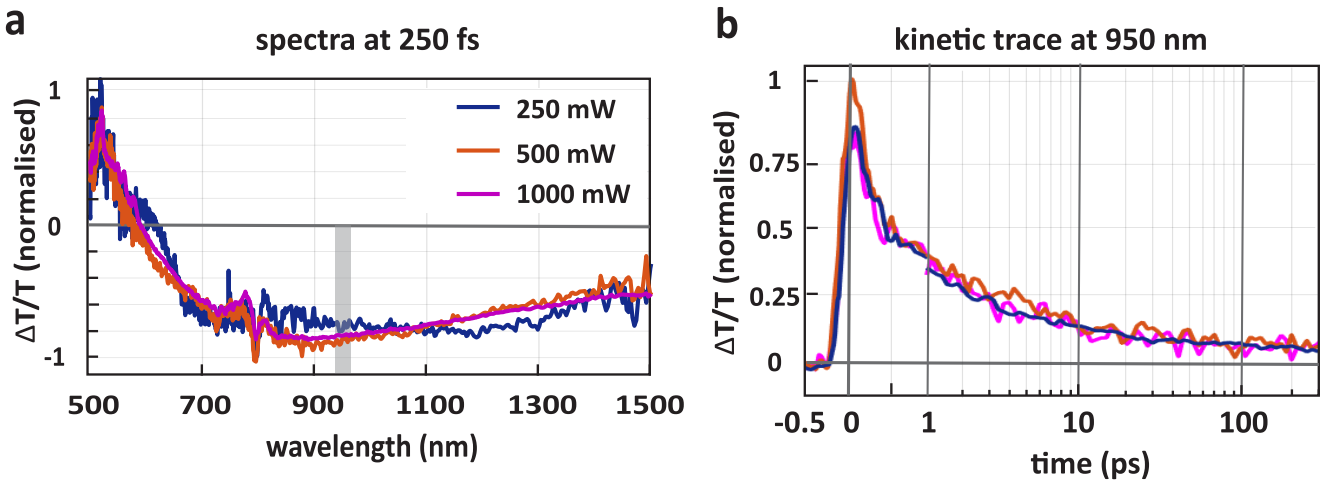

Figure 6.2: Transient absorption spectra and kinetics of eumelanin films are presented for a pump excitation wavelength of $532 \mathrm{~nm}$. Panel a shows normalised spectral slices taken at the same time point $(250 \mathrm{fs})$ for 3 different excitation pump powers. Panel $\mathrm{b}$ shows the decay kinetics (normalised to be the same value at $1 \mathrm{ps}$ ) taken at $950 \mathrm{~nm}$ indicated in panel a by the grey bar.

here as the energy of the pulse per unit area, where energy = average power/pulse repetition rate. From this plot we see that the spectral shape of the signal is the same for each fluence. Panel $b$ shows the normalised decay kinetics averaged at $950 \mathrm{~nm}$ over $\mathrm{a} \pm 10 \mathrm{~nm}$ range, indicated in panel $a$ as the grey bar. The kinetic traces are the same for each pump power, which indicates the fascinating capability of the eumelanin macromolecule to rapidly disperse photon energy even at extreme fluence levels. Further analysis of eumelanin's fluence dependent spectra and kinetics are discussed in section 6.5 of this chapter.

\subsubsection{Determining the optical Raman pump frequency for eumelanin}

Of interest to FSRS is the placement of the Raman pump in terms of wavelength. Ideally, the Raman pump should not be absorbed by the sample, that is not centered in an area of strong absorption as described by the UV-Vis spectra, but placed in the region of the PIA band. This is because achieving resonance with the excited state can enhance the stimulated Raman signal which is improved by $\sim 100$ times when on resonance with a material's PIA band [55/86] 89$]$. The excitation and Raman pump wavelengths are therefore selected to maximise both the excited state population generated from the excitation pump, and the Raman gain yield. The UV-Vis absorption and transient ab- 


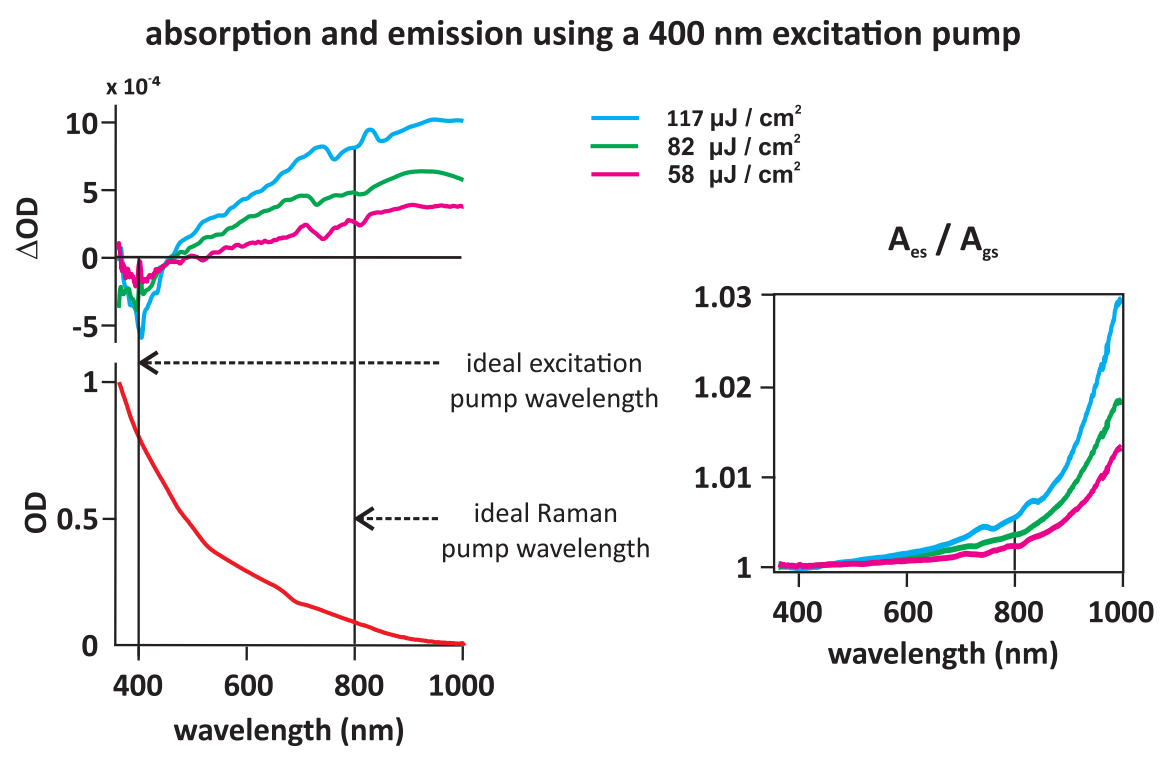

Figure 6.3: The comparison of the excited and ground state absorptions for eumelanin films: ideally the Raman pump is located in wavelength at an energy where melanin doesn't absorb in the ground state, but has a strong photo induced absorption band. In this case the closer to the IR the better, although limitations of the whitelight generation, Raman pump power generation and camera spectral range (limited towards the IR) make it optimal to place the Raman pump at $800 \mathrm{~nm}$, with a Raman spectral range of $800-1000 \mathrm{~nm}$ covering the main area of interest in eumelanin's FSRS dynamics

sorption spectra are used to determine the optimal wavelength location for each pump. Figure 6.3 shows a comparison of the absorption spectra with the TA spectra, with the ideal wavelength positions for the excitation and Raman pumps indicated. In these experiments ideally the Raman pump wavelength is tuned to the IR and the excitation pump is tuned to wavelengths of $400 \mathrm{~nm}$ and below to provide a strong ground state absorption.

The Raman pump generated by the bandwidth compressor detailed in chapter 3 is optimised for a $400 \mathrm{~nm}$ pump wavelength - for eumelanin we note that this is not ideal, in fact, it's less than ideal given that eumelanin pigment strongly absorbs there and the PIA band is in the IR, peaking at around $954 \mathrm{~nm}$ as shown by the TA data. Experimentally, applying the $400 \mathrm{~nm}$ Raman pump to eumelanin films proves ineffective, with the ground state intensity presenting as less than $10^{-5} \mathrm{~m}$ counts on the spectrometer not intense enough to be resolved above the noise level despite rigorous processing and 
significant shot averaging. Comparing the absorption spectra with the TA spectra, the ideal wavelength position for the Raman pump, as shown in figure 6.3, is in the IR in order to achieve resonance enhancement with the PIA band. Tuning the Raman pump wavelength to $800 \mathrm{~nm}$ achieves this. The excitation pump tuned to wavelengths of 400 $\mathrm{nm}$ and below allows for a strong ground state absorption, creating an excited state population for the stimulation of Raman gain photons, allowing dynamic vibrational kinetics to be resolved.

\subsection{Experimental configuration}

\subsubsection{FSRS experimental configuration}

To interrogate eumelanin's excited state relaxation dynamics, two excitation pumps in the visible $(400 \mathrm{~nm})$ and the UV $(267 \mathrm{~nm})$ regions are used. The samples are prepared as films and dispersions to make comparisons between different environments. In this section the sample preparations and optical system configurations are detailed.

\subsubsection{Sample preparation}

\subsubsection{Eumelanin film preparation}

To prepare thin films of eumelanin, synthetic eumelanin powder (Sigma Aldrich M8631) is suspended in aqueous ammonia solution (Simga-Aldrich ACS reagent, $28.0-30.0 \%$, $\mathrm{NH}_{3}$ base; 320145) at $60 \mathrm{mg}$ powder per $1 \mathrm{~mL}$ aqueous ammonia. The suspension is then sonicated for 25 minutes, and pipetted onto a quartz substrate at an approximate volume of $120 \mu L$ for spin coating.

The substrate is accelerated at $665 \mathrm{rpm} / \mathrm{s}$ to $600 \mathrm{rpm}$ for 2 minutes in order to disperse the solution, and then accelerated at $665 \mathrm{rpm} / \mathrm{s}$ to $4000 \mathrm{rpm}$ for 30 seconds in order to evaporate the ammonia. In this technique, the basic $\mathrm{NH}_{3}$ ammonia solution scavenges protons from the acidic eumelanin molecule to form $\mathrm{NH}_{4}+$, causing eumelanin to become de-protonated. The de-protonated eumelanin dissolves well, as hydrogen bonds are formed between each molecule, without transfer of atoms (i.e. no chemical reaction takes place). During spin coating, the $\mathrm{NH}_{4}+$ ions transfer protons back to eumelanin ions eventually forming $\mathrm{NH}_{3}$, which then evapourates. Eventually all the $\mathrm{NH}_{4}+$ molecules are funnelled through this pathway, leaving eumelanin behind in a 

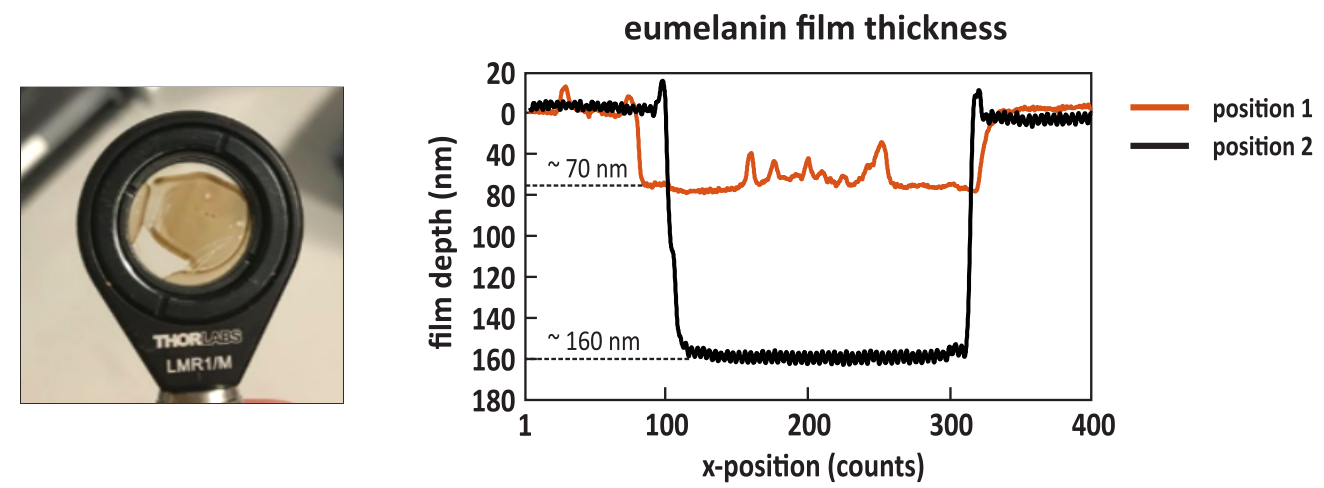

Figure 6.4: A typical eumelanin film is shown in the picture on the left panel of this figure. The thickness of a eumelanin film is measured at two different positions, by first scratching a line across the film using metal tweezers, and then running a stylus over the groove using a commercial Veeco Dektak 150 profilometer. The first position measures a depth of $\sim 70 \mathrm{~nm}$ and the second position measures a depth of $\sim 160 \mathrm{~nm}$. This highlights the in-homogenity of the film, and while the exact location of the beam path in the FSRS can't be determined, and average film depth of $\sim 100 \mathrm{~nm}$ can be assumed.

protonated, solid state form.

Each eumelanin film prepared is in-homogeneous in thickness. Figure 6.4 shows an example of one film measured at two positions using a Veeco Dektak 150 profilometer. The first position is measured to be $\sim 70 \mathrm{~nm}$ thick, and a second position on the same film is measured at $\sim 160 \mathrm{~nm}$. Without knowing the precise location on the film being probed by the laser pulses, an approximation of around $100 \mathrm{~nm}$ film thickness is assumed. FSRS data is collected in these experiments for two separate films and dispersions, with their UV-Vis absorptions shown in figure 6.5. The UV-Vis absorption is measured before and after each experiment, and the TA data is compared at the beginning and end of the experiment to check for film degradation caused by laser excitation. No such degradation is observed.

\subsubsection{Eumelanin dispersion preparation}

Eumelanin dispersions are prepared in aqueous ammonia in the same way as prepared for thin films, as described above. The UV-Vis results for both films and disper- 

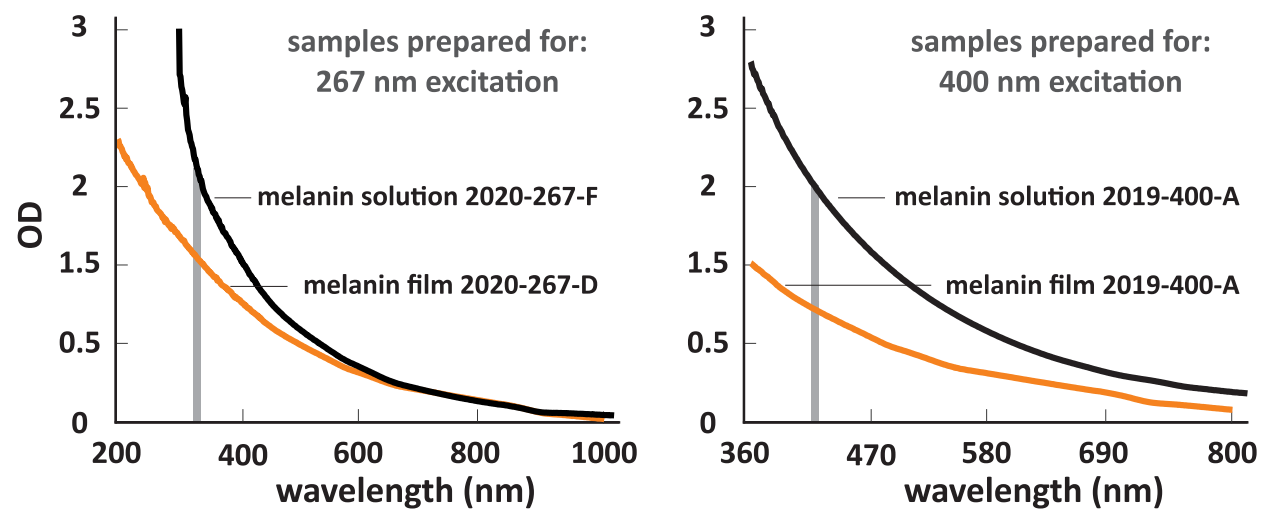

Figure 6.5: UV-Vis spectra taken for the eumelanin film (orange curves) and dispersions (black curves) used in these experiments are presented. Absorptions at 267 $n m$ are shown in the left hand panel, with 1.6 and 2.2 OD respectively, and 0.8 and 2 OD for the film and dispersion samples prepared for $400 \mathrm{~nm}$ excitation.

sions show good approximation within each sample set as shown in figure 6.5 for both preparations.

\subsubsection{Pump details and spectrometer configuration}

The narrowband Raman pump centered at $800 \mathrm{~nm}$ is generated from the fundamental using the spatial filtering method discussed in detail in section 3.8.2 of chapter 4.1. A pump energy of $1000 \mu \mathrm{J} / \mathrm{cm}^{2}$ is used with a $39 \mathrm{~cm}^{-1}$ bandwidth. For each experiment, the Raman pump configuration is set to be as close as possible to be the same compressed bandwidth and fluence conditions in subsequent experiments. Two excitation pump excitation wavelengths are used at $267 \mathrm{~nm}$ and $400 \mathrm{~nm}$, generated using the second and third harmonic setup described in section 4.2 .2 of chapter 4.1 . In the first experiment, a pulse energy of $3.5 \mu \mathrm{J}$, and a fluence of $1273 \mu \mathrm{J} / \mathrm{cm}^{2}$ is measured for the $267 \mathrm{~nm}$ excitation pump. For the second experiment using the $400 \mathrm{~nm}$ excitation pump, a pulse energy of $0.67 \mu \mathrm{J}$, and a fluence of $339 \mu \mathrm{J} / \mathrm{cm}^{2}$ is used. For the Raman pump tuned to $800 \mathrm{~nm}$, the spectrometer configuration is used as described previously in chapter 4.1. This is the same configuration as for configuration 1 used for FSRS measurement taken of DHI and DHICA described in chapter 5 (while configuration 2 of that chapter uses a $400 \mathrm{~nm}$ Raman pump). For comparisons between the eumelanin film measurements presented in this chapter and the DHICA measurements presented previously, the experimental configuration was set to be as close as possible to being under the same conditions, particularly in terms of the system's fluence, calibration 
and optical resolution.

\subsubsection{Data processing}

The previous chapter 4.1 details the data processing used to extract dynamic Raman gain signals, as well as TA spectra and kinetics. The TA dynamics are of particular importance in the data analysis of eumelanin, as the relaxation dynamics are used as spectral mask in the SVD analysis, a mathematical tool useful when isolating overlapping spectral signatures ${ }^{129 \mid 130]}$. Details of the SVD analysis, including the use of temporal masks, are described in section 4.5.5.1 of chapter 4 .

\subsection{Experimental results}

\subsubsection{Ground state Raman of eumelanin}

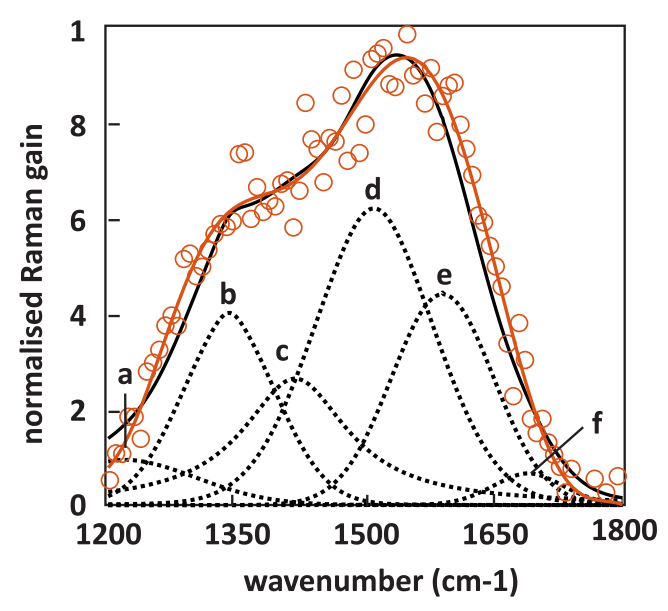

Figure 6.6: The broadband ground state Raman show two main peaks around $1360 \mathrm{~cm}^{-1}$ and $1590 \mathrm{~cm}^{-} 1$, similar to amorphous carbon. These peaks can be assigned as " $\mathrm{D}$ " and G" like, that is, representation of the dis-ordered and ordered structured within the molecule. A fitting is performed using 6 pseudo-Voigt functions labelled as the black dashed curves $a-f$, shown as the solid black curves shows a good fit to the raw data (orange circles). The solid orange line is a Matlab poly-fit to the raw data, independent of fitting. 


\subsubsection{Ground state Raman band assignments}

Due to its highly disordered nature, it is expected that the Raman bands for eumelanin are not sharp narrowband peaks as in typical organic materials, but rather a broad envelope consisting of a combination of overlapping vibrational modes close in frequency to each other ${ }^{[150+153]}$. The two broad peaks present in the ground state Raman spectrum of eumelanin are shown in figure 6.6. centered at $\sim 1360 \mathrm{~cm}^{-1}$ and $\sim 1590 \mathrm{~cm}^{-1}$, and can be modelled by a series of pseudo-Voigt functions peaking at expected frequencies based on density functional theory (DFT) ${ }^{[133]}$. A pseudo-Voigt function, also known as a Cauchy-Lorentz distribution, is a convolution of Gaussian and Lorentzian functions ${ }^{[154]}$. Usually in a polymeric material, the Raman peaks are modelled with Lorentzian functions, however due to the highly disordered nature of eumelanin leading to peak broadening, it is applicable to use pseudo-Voigt functions in the deconvolution analysis. Here we use literature values based on DFT analysis to fix the peak frequencies for each of the functions, with the amplitudes and FWHM bandwidths converged upon in a step-wise analysis. Our pseudo-Voigt analysis of eumelanin's ground state Raman spectrum shows good agreement with the literature ${ }^{[155]}$.

\begin{tabular}{|l|l|l|}
\hline \multicolumn{3}{|c|}{ peak positions of pseudo-Voigt functions for eumelanin's ground state } \\
\hline peak & central peak & mode assignment \\
\hline$a$ & $1220 \mathrm{~cm}^{-1}$ & C-OH phenolic stretching; C-O stretching in the COOH group \\
\hline$b$ & $1341 \mathrm{~cm}^{-1}$ & C-N aromatic stretching in the indole subunit \\
\hline$c$ & $1418 \mathrm{~cm}^{-1}$ & N-H bending \\
\hline$d$ & $1510 \mathrm{~cm}^{-1}$ & C=N stretching in the SQ subunit \\
\hline$e$ & $1590 \mathrm{~cm}^{-1}$ & C=C stretching of the indole subunit \\
\hline$f$ & $1690 \mathrm{~cm}^{-1}$ & C=O stretching in the IQ and SQ subunits \\
\hline
\end{tabular}

Table 6.1: Central peak locations, and their corresponding mode assignments, are presented here for the 6 main peaks derived from a pseudo-Voigt function fitting of eumelanin's broad-band, ground state peaks.

Figure 6.6 shows the experimental ground state spectrum for eumelanin as orange circles, fit with a 6 component poly-fit function shown as the orange curve. The spectrum is fitted using six pseudo-Voigt functions $(a-f)$, shown as dashed black curves, combined as the solid black curve. This fitting analysis shows good agreement with 
the experimental data poly-fit. The fitting algorithm is generated using a home-built Maltab function shown in appendix $B$, with the variables for each functions given in table E.1 of the same appendix.

The peak features of each pseudo-Voigt function are taken from literature and represent differing vibrational modes in the various monomer (and oligomer) groups assigned as $a$ to $f$, shown in table 6.1 155/156]. These peaks may be grouped into " $G$ " and " $\mathrm{D}$ " like modes in comparison with those observed in amorphous carbon, where " $\mathrm{D}$ " indicates lower frequency "disordered" structural vibrations, and "G" indicates higher energy ordered, graphitic like structures. The 3 higher vibrational peak frequencies identified in eumelanin from the pseudo-Voigt modelling are double bonded vibrations, while the 3 lowest frequencies are single bonded vibrations. This comparison between eumelanin and amorphous carbon in the ground state is further detailed in section 6.4 .2

\subsubsection{Comparisons between eumelanin and amorphous carbon ma- terials}

In amorphous carbon, both $s p 2$ and $s p 3$ hybridised carbon groups are present $157-160]$. The sp2 aromatic rings subunits are covalently bonded by 3 bonds ( 2 single and 1 double) in a planar geometry. The $\pi$ orbital can than delocalise and form conjugated stacks, much like pancakes stacked on top of each other. The sp3 subunits are "defects" formed at the outer boundary of the molecule, and have 4 single bonds attached in a tetrahedral configuration. The comparison of the intensity of the " $\mathrm{D}$ " and "G" bands reveals the level of "disorder" in the molecule. For example, a perfect graphene sheet would only exhibit an intense "G" band.

The " $\mathrm{D}$ " peak can be assigned to a breathing mode of the carbon rings at the structural boundary as shown in the top left of panel "a" in figure 6.7, while the "G" peak can be assigned to the in-plane bond-stretching motion of pairs of $s p 2$ atoms, present along a chain structure, as shown in the bottom left panel "a" of figure 6.7. The " $\mathrm{D}$ " band in carbon based materials has a larger Raman cross section, and thus this vibrational band appears more intensely than the "G" band. Comparison of the two bands therefore is the best way to illicit information about the ordered state of different carbon materials.

Panel $b$ in figure 6.7 shows the two key vibrational modes. In amorphous carbon, two 


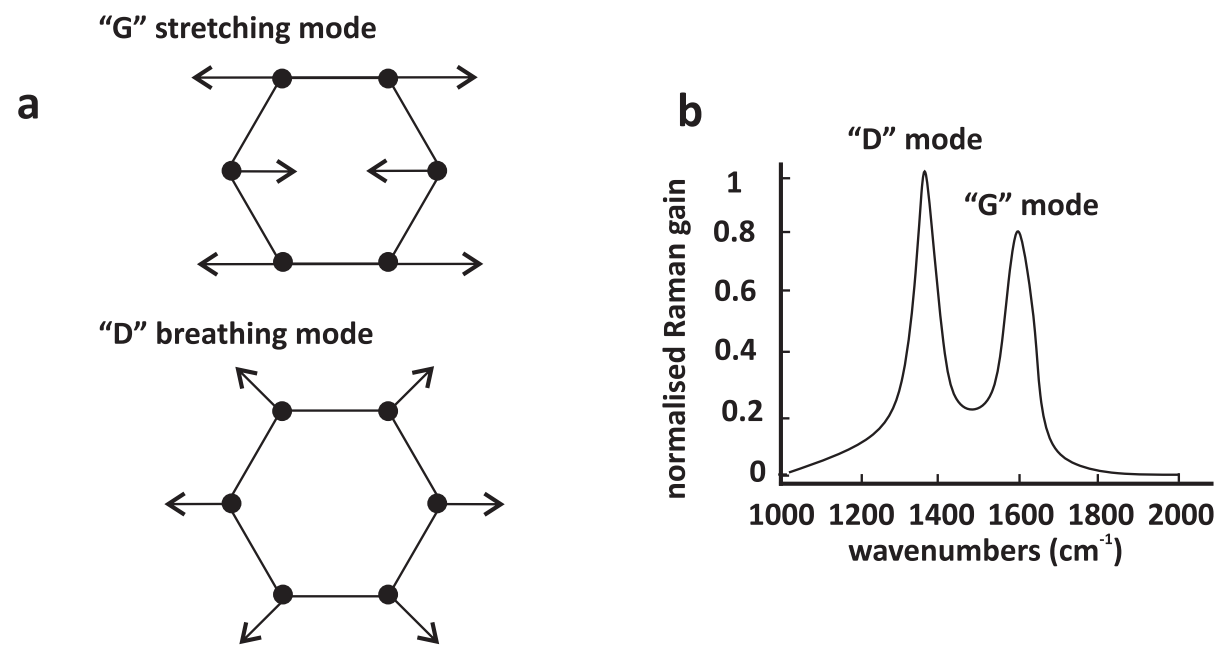

Figure 6.7: The key Raman modes of amorphous carbon shown in panel $b$ can be described at " $\mathrm{D}$ " like and "G" like. This refers to disordered and ordered structures respectively. For example, a perfect graphene sheet would have only a "G" band in its Raman spectrum. Panel $a$ shows the two related vibrational modes, where the top figure shows the "G" stretching mode of the $s p 2$ carbon rings formed across an ordered lattice structure, while the bottom figure shows the " $\mathrm{D}$ " breathing mode of $s p 3$ carbon rings located at the edges of the lattice.

main peaks are present in the Raman spectrum at $1355 \mathrm{~cm}^{-1}$ - the " $\mathrm{D}$ " peak $-1581 \mathrm{~cm}^{-1}$ - the $\mathrm{G}$ peak. This model of carbon in terms of order and disorder is of interest to our studies of eumelanin as, being a primarily carbon based material, eumelanin's vibrational signatures in the ground state show similar " $\mathrm{D}$ " and " $\mathrm{G}$ " bands, albeit with a broader spectral envelope. Observing changes to these bands in terms of frequency position, intensity and bandwidth, we can make comparisons to the behaviour of eumelanin with pure carbon materials.

\subsubsection{Resonance Raman analysis}

In a heterogeneous material, as the Raman pump wavelength comes into resonance with chromophores that differ in the size in terms of their conjugation lengths, their Raman gain signals become resonantly enhanced, producing characteristic shifts, known as 'dispersion', of the observed Raman bands ${ }^{[161162]}$. Experimentally, the strongest Raman peak of eumelanin centred at around $1590 \mathrm{~cm}^{1}$ red-shifts with increasing Raman excitation wavelength energy. 

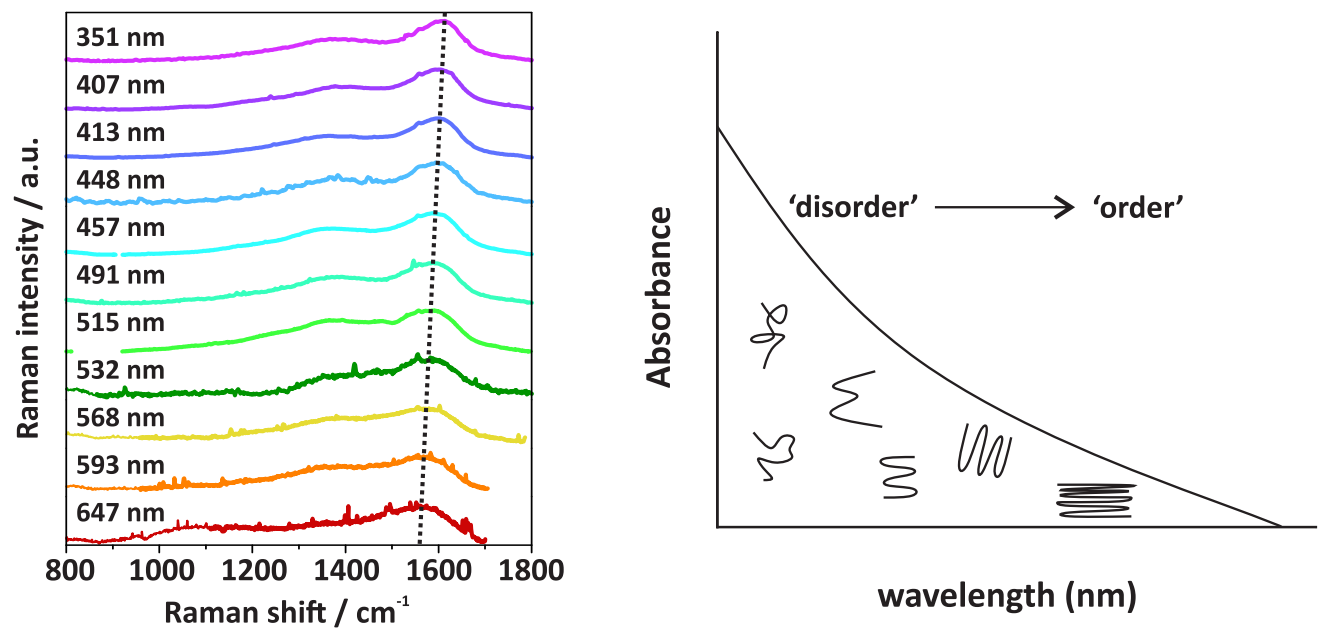

Figure 6.8: The ground state Raman spectra is presented for eumelanin films, in collaboration with Otago University. 11 Raman pump excitation wavelengths are used under the same experimental conditions, showing a distinct blue-shift of the main ground-state Raman band peak centered around $1590 \mathrm{~cm}^{-1}$. The right hand panel shows a schematic of eumelanin's absorption spectra, which the conjugated chromophore lengths of eumelanin's disordered structure evolving from "disorder" to "order" at lower energy wavelengths.

Figure 6.8, collected by collaborators at the University of Otago ${ }^{[163}$, agrees well with literature showing the peak shifting approximately $50 \mathrm{~cm}^{-1}$ from $1560 \mathrm{~cm}^{-1}$ to 1610 $\mathrm{cm}^{-1}$ with excitation wavelength increasing from $647 \mathrm{~nm}$ to $351 \mathrm{~nm}$. This study provides an insight into the resonance nature of eumelanin as being dependent on the Raman pump wavelength. It also provides support to the argument of chromophores of overlapping spectral properties being individually excited dependent on excitation wavelength. It is expected that chromophores of increasing conjugation length and therefore a higher level of "order" will give rise to more phonon like higher frequency modes, which is shown in the data.

\subsubsection{Excited state vibrational mechanisms of eumelanin}

This section presents FSRS data collected for eumelanin films, for two excitation wavelengths in the UV $(267 \mathrm{~nm})$ and the visible $(400 \mathrm{~nm})$ regions. The FSRS surfaces are 


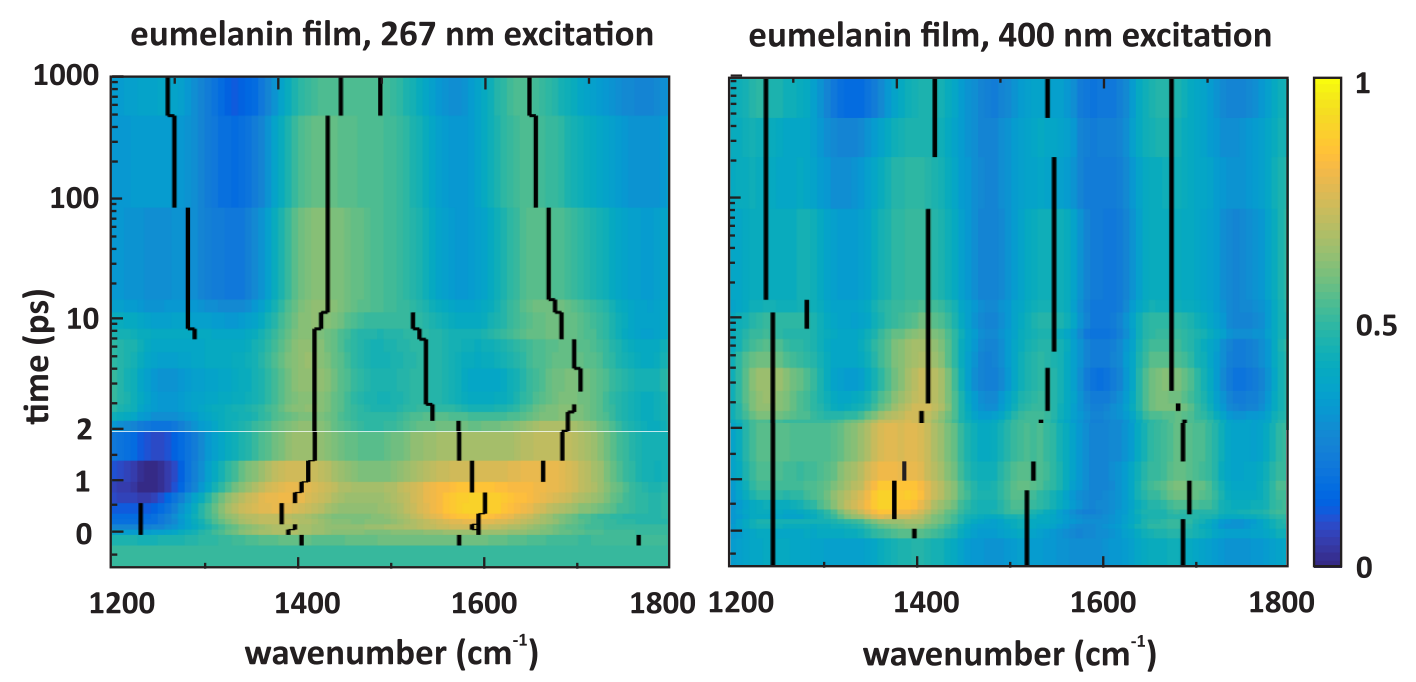

Figure 6.9: FSRS surface of melanin films for two excitation pump wavelengths. The surface in the left panel is for a $267 \mathrm{~nm}$ excitation pump, and the surface in the right panel is for a $400 \mathrm{~nm}$ excitation. The differential analysis performed on each surface reveals the peak locations for each spectral slice as a function of time, and are shown as the overlayed black curves in each surface.

analysed using a derivative function as introduced in section 4.5.5.2 of chapter 4 . The surfaces are also analysed using singular value decomposition (SVD) as described in section 4.5.5.1 of chapter 4 , with these results being presented in appendix $\mathrm{F}$

Initial observations of the raw data FSRS surfaces presented in figure G.1 show some interesting comparisons. The frequency peaks calculated from the differential analysis are overlayed on each surface, as the black curves. These curves are plotted separately from the FSRS surfaces as a function of time in figure 6.10, with panel $a$ being for 267 $\mathrm{nm}$ excitation and panel $b$ being for $400 \mathrm{~nm}$ excitation. First, similarities between the film surfaces of figure G.1 are apparent in terms of their peak intensity rise and decay times, with kinetics presenting on $p s$ timescales. Additionally, by comparing the peak curves in figure 6.10, we see frequency shifts over time for both excitation wavelengths. Given the evidence of the TA data presented in section 6.2.1. it is expected that for different excitation wavelengths, different chromophores will be excited and hence the vibrational peak frequencies of the excited state will also differ. If ESPT is a preferred deactivation pathway, this should be observed in both the film surfaces.

A similar analysis of the FSRS surfaces of eumelanin prepared as a dispersion in ammo- 

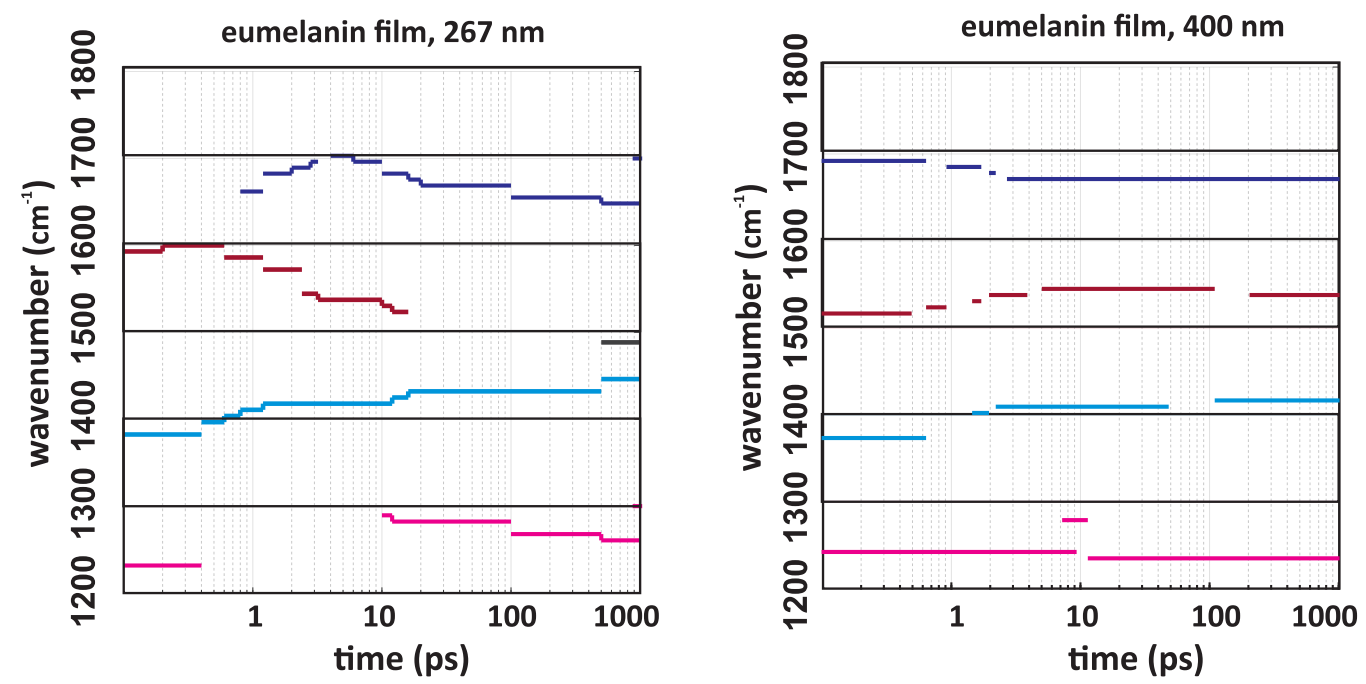

Figure 6.10: Comparison of eumelanin films at $267 \mathrm{~nm}$ and $400 \mathrm{~nm}$ excitations. The peak frequencies are plotted as a function of time, and colour coded to note similarities between the two surfaces. It is apparent that for both excitation pump wavelengths there are frequency shifts occurring.

nia, rather than in a film, were also conducted. Referencing this data to the molecule's behaviour in Nature is challenging, as the extreme, basic $\mathrm{pH}$ conditions of the solvent $\left(p K_{a}=37\right)$ mean that the molecule will be de-protonated upon experimentation. In the dispersion preparation, the FSRS surfaces present kinetics with a more uniform and monotonic rise and decay of vibrational modes peaking around $1 \mathrm{ps}$, with no obvious shifting of peak frequencies. These data surfaces, and differential analysis, are presented in appendix $\mathrm{G}$.

\subsection{Discussion}

Two hypotheses are explored in this section. The first hypothesis is that excitations of eumelanin decay via localised states, with evidence presented in the TA data and comparison of the ground and excited state Raman signatures. The second hypothesis is that ESPT is a deactivation pathway for eumelanin films, with evidence presented comparing the dynamics of eumelanin's FSRS surface against that of DHICA's vibrational signatures. 
Hypothesis 1: Excitation's in eumelanin decay via localised states.

This hypothesis encompasses the idea that while eumelanin's ground state optical properties reflect that of a disordered, heterogeneous material, in its excited state, specific excitations are activated and then de-activated via localised pathways. The heterogeneity of eumelanin's ground state optical signatures have been already introduced in this research, discussed in chapter 1 . To summarise, in the ground state, eumelanin exhibits a broadband monotonic, featureless UV-Vis absorption spectrum which can be modelled mathematically as several Gaussian functions of overlapping absorption bandwidths, representative of individual chromophores. Eumelanin's ground state emission is equally unique in that emission frequencies are excitation wavelength dependent, violating Kasha's law ${ }^{[33]}$. The emission spectrum also disobeys the Mirror Image rule which state that the emission and absorption spectral band shapes should mirror each other ${ }^{[164]}$, but in eumelanin's emission spectrum the peak shapes are Gaussian in nature, quite unlike the broad band absorption spectrum ${ }^{[18]}$.

Eumelanin's ground state Raman spectrum presented in section 6.4.1 displays two broad band peaks, similar to that of amorphous carbon, indicating a molecule that is heterogeneous and disordered by nature. The spectrum can be modelled by 6 pseudoVoigt functions with different frequency positions and bandwidths. These functions represent several vibrational modes with overlapping frequencies, indicating that the various chromophores in eumelanin have differing conjugation lengths, or differing amounts of structural "order" versus "disorder". For example, a higher frequency phonon mode has a longer conjugation length and a higher level of structural "order" compared to a lower frequency bending mode. Resonance Raman studies of eumelanin in the ground state (presented in section 6.4.2.1) further support this idea, as the main spectral peak at $1590 \mathrm{~cm}^{-1}$ shifts with Raman pump wavelength, indicating wavelength dependent vibrational resonances. The excited state FSRS surfaces of eumelanin, however, exhibits a richer pattern of frequencies more indicative of molecular-type modes. The localised nature of excited state relaxation is apparent by the comparison of the ground state and excited state data spectra of eumelanin shown in figure 6.11. Two broad bands that are sensitive to conjugation length are observed in the ground state (black circles fitted with solid black line), whereas the excited state surface spectral time-slices exhibits (orange to red to black curves) show more distinct peak frequencies evolving over time. At early times (orange curves) the excited state 


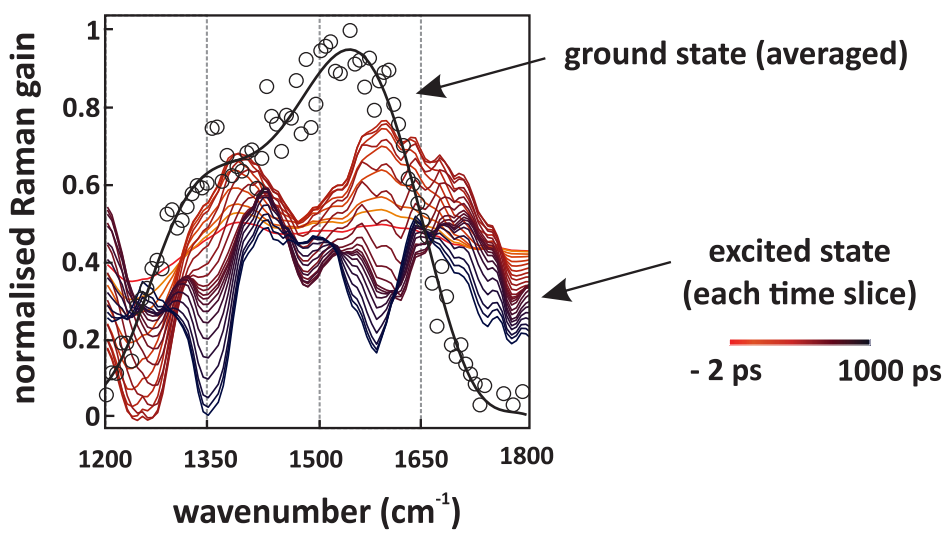

Figure 6.11: The averaged ground state Raman spectrum is shown as the black circles, fitted with the curve shown as the solid black line. Each time slice of the excited state FSRS surface is plotted on the same figure with the time points from -2 ps to 100 ps plotted as yellow - orange - black curves.

surface shares similarities with the broad band peaks of the ground state, which could be from a resonance enhancement of all modes being initially excited. By later times (black curves), different vibration frequencies are apparent, which shows that the excited state vibrational decay is deactivated through selective, localised channels.

The second supporting evidence for this hypothesis is a lack of fluence dependence in eumelanin's TA spectra. This data presented and discussed in figure 6.2 of section 6.2.1. where 3 different excitation pump powers are used in separate experiments on the same eumelanin film (with all other experimental conditions the same). Here we see a lack of dependence on fluence in two ways; the normalised spectra have the same GSB and PIA spectral shapes, and the decay kinetics of the PIA band for each power are also the same. This indicates that excitations decay in a localised way, rather than being sufficiently mobile to interact at high density. In summary, both the TA and excited state Raman spectra, along with supporting evidence of the resonance Raman analysis, strongly suggest that there are indeed localised pathways for energy de-activation in the eumelanin macro-molecule.

This conclusion leads us on to thinking about the non-radiative relaxation mechanisms of the localised building blocks of eumelanin, and whether these signatures can be mapped onto those of the macro-molecule. It is this idea that is explored in the second part of this discussion. 


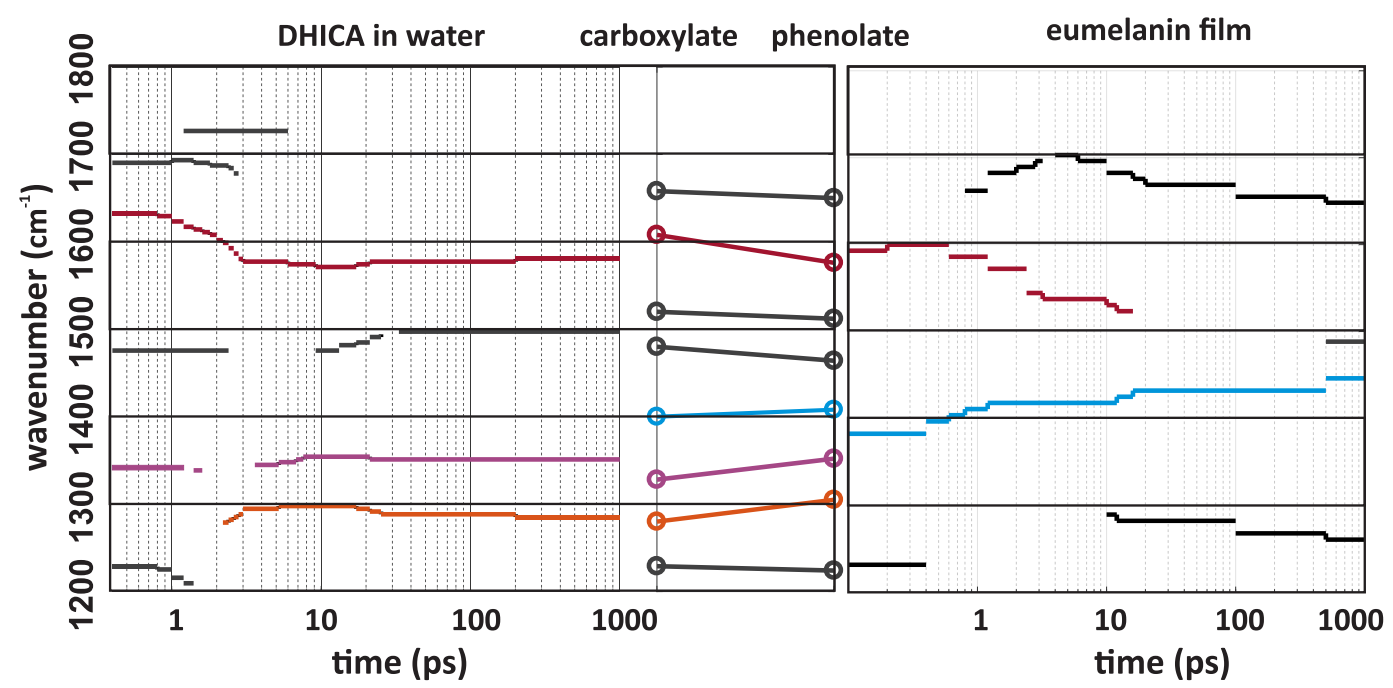

Figure 6.12: The overlayed black curves of figure 6.13 for DHICA in water and eumelanin films are compared side by side in the left and right panels, with the DFT peak shifts presented in the middle. Comparisons of the two peak shifts are colour coded to show the similarities between them. Of particular interest is the maroon curve at around $1600 \mathrm{~cm}^{-1}$, which agrees well with the DFT frequency shifts, and is present in both eumelanin and the DHICA monomer.

Hypothesis 2: Excited state proton transfer (ESPT) as observed in DHICA is a key nonradiative relaxation mechanism of eumelanin pigment.

In this discussion, comparisons of the vibrational spectra and excited state decay pathways are made between DHICA and eumelanin films under $267 \mathrm{~nm}$ excitation. Chapter 5 shows strong evidence of excited state proton transfer (ESPT) occurring in DHICA dissolved in water, with the analysis of peak shifts in $p s$ timescales matching those of the peak shifts calculated using DFT analysis of the excited state DHICA carboxylated anion and its phenolate di-anion structures.

The peak positions calculated from the FSRS surfaces of eumelanin films using a differential analysis are plotted previously in figure 6.10 for 2 excitation pump wavelengths in the UV $(267 \mathrm{~nm})$ and visible $(400 \mathrm{~nm})$ regions. Different excitation wavelengths should selectively excite different chromophores in the material, and so the FSRS vibrational peaks, and any peak shifts, are expected to differ between the two experi- 
ments. Here we see that, while the vibrational frequencies are not identical, peak shifts are occurring in both FSRS surfaces, and on similar, fast time scales.

To compare the FSRS surfaces of eumelanin with the DHICA molecule, we look at the $267 \mathrm{~nm}$ excitation data only, in order to ensure that the same chromophores are under excitation. It is noted here that a comparison using $400 \mathrm{~nm}$ excitation is not possible, as DHICA does not have an absorption band at this wavelength and thus no excited state population will be created. The Raman pump wavelengths used in these experiments are different however, with an $800 \mathrm{~nm}$ IR Raman pump being used in the eumelanin experiments, and a $400 \mathrm{~nm}$ Raman pump being used in the DHICA experiments. This discrepancy is purposeful, as the PIA bands for each material are in the IR and the visible regions respectively, meaning resonance enhancement will be achieved in each case. In terms of sample preparations, when comparing the two mechanisms we want the samples to be both present in a water bath, where ESPT is allowed. This is a challenge for eumelanin, as it does not dissolve in water. Instead, we can make the assumption that water is present in the eumelanin film despite being in the condensed phase. We believe this to be a valid assumption given the prevalence of polar groups at the edge of the molecule, and the spin coating process being performed in open air.

It can be seen when comparing the raw FSRS surfaces of eumelanin under UV excitation (figure G.1 1 ) and DHICA (figure 5.11 ), that the excited state vibrational modes activated in eumelanin upon UV excitation are less distinct in frequency than those of its DHICA monomer (but are still localised bands in comparison with eumelanin's vibrational ground state). Figure 6.12 shows the comparison between the DHICA and the eumelanin FSRS surface peaks at $267 \mathrm{~nm}$ excitation. Of interest when comparing the frequency peaks only, is the shifting of peak frequency positions observed in DHICA and if they are also present in the eumelanin FSRS surface. This similarity is particularly apparent for the frequency peak around $1600 \mathrm{~cm}^{-1}$, shown as the red curves in each panel, where a significant blue-shift is apparent. The excited state vibrational modes associated with this frequency shift are shown in figure 5.14 of section 5.4.3.3. chapter 5. Similarly, around $1700 \mathrm{~cm}^{-1}$ (top black curves) there are some similarities between the frequency shifts in both eumelanin and DHICA with the DFT analysis, these however occur on differing time scales and may not be conclusive. In the eumelanin surface alone, we also see the presence of a shifting peak (light blue curve) around $1400 \mathrm{~cm}^{-1}$ which matches well to the DFT analysis, although this peak is not observed in the DHICA data. 

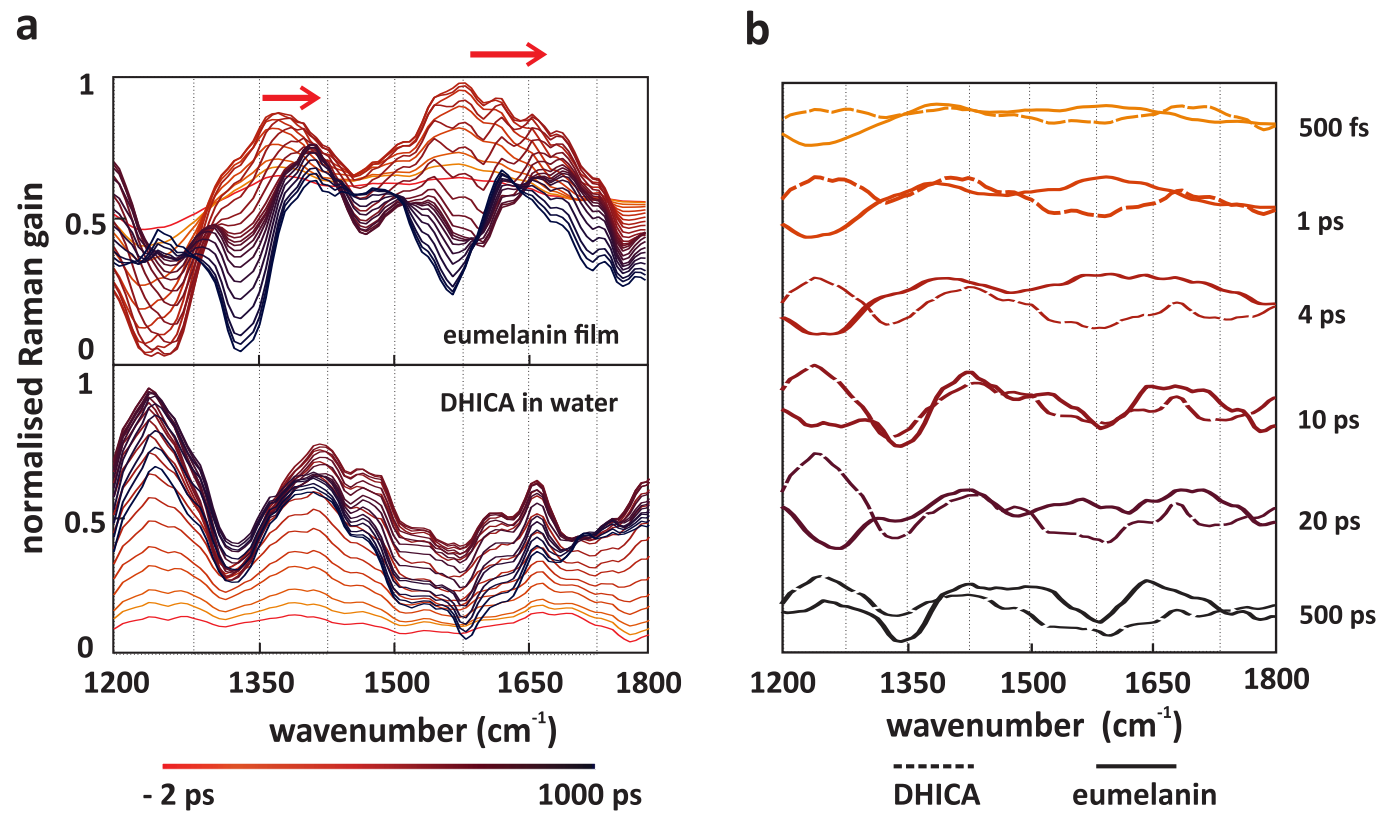

Figure 6.13: Panel $a$ shows the spectra for all time points from negative time (in orange) proceeding to $1000 \mathrm{ps}$ (in black) for the eumelanin film (top) and DHICA in water (bottom). Panel $b$ shows 6 spectral slices taken at individual times, from $500 \mathrm{fs}$ to $500 \mathrm{ps}$. The dashed curves are the DHICA spectra, while the solid curves are taken from the eumelanin FSRS surface. We see that the while initially the main peak positions are spectrally offset - on longer timescales they appear to mirror each other, seen particularly in the high energy vibrational band around $1250 \mathrm{~cm}^{-1}$ (black curves).

Figure 6.13a shows the individual spectral slices from $-2 \mathrm{ps}$ to $1000 \mathrm{ps}$ taken from the eumelanin (top panel) and DHICA (bottom panel) surfaces. Panel b of the same figure shows just 6 of this time slices overlayed on top of each-other for eumelanin and DHICA, progressing from $500 \mathrm{fs}$ to $500 \mathrm{ps}$. From this data we see that the initial excited state vibrations of eumelanin shift in frequency (indicated by the red arrow) from an initial vibrational state, to one that is similar to DHICA. Comparing the peaks of the black curves in the top plot of panel $a$, the frequency positions are close to those to the black curves in the lower plot. This could indicate the presence of the DHICA vibrational signature in the eumelanin molecule at later times after excitation. It is clear in panel $b$ of the figure that by $500 \mathrm{ps}$ the spectral slice for each material matches the other in terms of frequency positions. 
Further interest studies of eumelanin outside of these key hypotheses are next presented.

\subsection{Heating effects in eumelanin films}

\subsubsection{Temperature dependence}

Based on rapid non-radiative relaxation, we know that laser excitation must heat the eumelanin samples on a fast time scale during experimentation. It is possible that heating has an effect therefore on eumelanin's vibrational signatures as observed in carbon materials $157-159 \mid 165[166]$. One explanation for peak shifting is that it is a consequence of heating, rather than necessarily a signature of the mechanism. It is hard to reference this proposition, as studies into thermal effects are essentially observing the annealing, or "cooking", of the material causing irreversible structural changes. Comparisons between eumelanin's ground state Raman spectra, and other carbon structures can still be made. It is documented in literature that the evolution of amorphous carbon into graphite due to annealing occurs at around $400{ }^{\circ} \mathrm{C}$ to $800{ }^{\circ} \mathrm{C}$. Blue-shifts of carbon's ordered " $G$ " peak (as discussed in section 6.4.2), and changes to the " $G$ " to " $D$ " peak bandwidth ratio can be accounted for by conversion of the molecule from a disordered structure to an ordered one, where bond re-organisation reduces the number of $s p 3$ structures. Upon heating up to $552{ }^{\circ} \mathrm{C}$, the $\mathrm{G}$ band of amorphous narrows and blue shifts by $78 \mathrm{~cm}^{-1[165]}$.

The following section first estimates the amount of heat imparted to the material from the pump lasers during experimentation, and then calculates the time taken to return the heated molecule to room temperature via dissipation through the substrate (or cuvette). We then look at the effect of temperature on eumelanin films in the ground state, to see if any similar observations are observed upon annealing using a temperaturedependent spontaneous Raman study.

\subsubsection{Estimating the temperature increase upon laser excitation}

The heat increase, $Q$, of the eumelanin sample upon laser pump excitation, can be calculated by measuring the laser power into the sample, $P_{\text {in }}$ and the laser power after the sample $P_{\text {out }}$. We use the area being excited, $A$, based on the laser spot size $s$, and the volume $v$ being excited calculated using the sample pathlength (assuming the ex- 
citation volume is a cylinder). Because two pump pulses are heating the sample, their excitation energies $E_{\text {excitation }}$ and $E_{\text {Raman }}$ are assumed to be instantaneous and arriving at the same time. This can be justified as the heat dissipation time of each laser pulses is shown to be longer than the longest measured $\Delta T$ delay between each pump arriving. Eumelanin's density $\rho$ is measured at $\sim 1700 \mathrm{~kg} / \mathrm{m}^{3}$, with its thermal conductivity $K$ approximated between $0.2 \mathrm{~W} / \mathrm{mK}$ to $2.2 \mathrm{~W} / \mathrm{mK}$, based on thin films of amorphous carbon. Thermal conductivity of amorphous carbon thin films ${ }^{1166}$. Similarly, eumelanin's heat capacity $C$ can be approximated as $710 \mathrm{~kJ} / \mathrm{kgK}$ based on amorphous carbon ${ }^{[167]}$.

Equation 6.1 shows the amount of energy being absorbed by first calculating the energy transfer into the sample as

$$
\Delta E=\frac{P_{\text {out }}-P_{\text {in }}}{\text { reprate }},
$$

with reprate being the laser repetition rate. Using the volume $v$ calculated from the area and pathlength illuminated, and the density $\rho$ of eumelanin, the mass $m$ illuminated by the laser pulses can be calculated as

$$
m=\rho * v,
$$

for each laser pulse. Combining the two laser pulse energies $E_{\text {excitation }}$ and $E_{\text {Raman }}$, and using eumelanin's heat capacity $C$, the change in temperature $\Delta T$ of the sample can then be calculated as

$$
\Delta T=\frac{E_{\text {excitation }}+E_{\text {Raman }}}{m * C} .
$$

Once the temperature increase arising from the "instantaneous" absorption of the laser pulse energies is calculated, the conductive heat transfer $Q$ dissipated through the substrate (or solvent) can be determined using the thermal conductivity $K$ of eumelanin (approximated for an amorphous carbon thin film as described above), volumetric properties film thickness $d$ and surface area $A$, and finally, the change in temperature $\Delta T$, as shown in figure 6.14. This is calculated as 


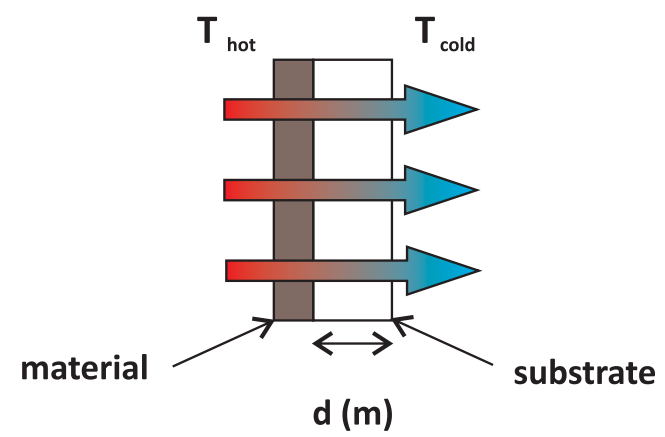

Figure 6.14: The direction of heat dissipation is shown by the arrows from $T_{h o t}$ to $T_{\text {cold }}$ through the material sample and the substrate, of thickness $d$. The initial temperature rise is assumed to be instantaneous using a temporally short pulsed laser as the heating source, and that all heat is dissipated through the substrate.

$$
Q=\frac{K * A * \Delta T}{d}
$$

It is then straight forward to extract from $Q$ the time for dissipation for both pulses arriving together as $E_{\text {total }} / Q$, assuming heating instantaneously the sample volume, by dividing the total energy dissipated by the conduction heat transfer rate, given as a function of temperature. To accurately reflect the heat transfer rate as being dependent on the temperature of the material, we integrate the temperature change over time as a function of the heat transfer rate (assuming instantaneous heating in the first instance due to the absorption of the short laser pulses). The absorbed heat is assumed to be entirely dissipated to the substrate (in the case of a film) or the solvent (in the case of a dispersion), hence the thermal conductivity of fused silica as the substrate $(2 \mathrm{~W} / \mathrm{mK})$ is used for eumelanin films, and for eumelanin dispersions, the thermal conductivity of the ammonia solvent $(0.3139 \mathrm{~W} / \mathrm{mK})$ is used in place of the substrate.

Table 6.2 lists the calculated temperature rises and dissipation times for each experimental configuration. For the film under $267 \mathrm{~nm}$ excitation, and $800 \mathrm{~nm}$ Raman pump excitation (as described in 4.1), a temperature increase of $\sim 201^{\circ} \mathrm{C}$ was calculated, with a dissipation time of $\sim 36 \mathrm{~ns}$. The increased power of the $400 \mathrm{~nm}$ excitation pump for the second configuration sees a greater rise in temperature at $\sim 302{ }^{\circ} \mathrm{C}$, with a longer dissipation time of $54 \mathrm{~ns}$. For dispersions at the same conditions, under $267 \mathrm{~nm}$ excita- 


\begin{tabular}{|l|l|l|l|}
\hline \multicolumn{4}{|c|}{ calculated temperature rise for films and dispersions } \\
\hline material & excitation power & temperature rise & dissipation time \\
\hline eumelanin film & $0.25 \mathrm{~mW}(267 \mathrm{~nm})$ & $201^{\circ} \mathrm{C}$ & $36 \mathrm{~ns}$ \\
\hline eumelanin film & $0.5 \mathrm{~mW}(400 \mathrm{~nm})$ & $302^{\circ} \mathrm{C}$ & $54 \mathrm{~ns}$ \\
\hline eumelanin solution & $0.25 \mathrm{~mW}(267 \mathrm{~nm})$ & $72^{\circ} \mathrm{C}$ & $2.0 \mathrm{~ns}$ \\
\hline eumelanin solution & $0.5 \mathrm{~mW}(400 \mathrm{~nm})$ & $109^{\circ} \mathrm{C}$ & $3.1 \mathrm{~ns}$ \\
\hline
\end{tabular}

Table 6.2: The calculated temperature rises for eumelanin films and dispersions are presented in this table for two different excitation powers, shown in column 2. Assuming instantaneous heating upon arrival of the pump laser pulses, the temperature rise of the eumelanin material is calculated in column 3. Assuming all heat is then dissipation through the substrate (in the case of the film), or the cuvette (in the case of the solution), the approximate heat dissipation time is shown in column 4 . Here we can see that it is on $n s$ timescales for the heat effects from the laser pulses to be dissipated, much shorter than the arrival of subsequent laser pulses.

tion pump excitation, and $800 \mathrm{~nm}$ Raman pump excitation a temperature increase of $\sim$ $721^{\circ} \mathrm{C}$ was calculated, with a dissipation time of $\sim 2 \mathrm{~ns}$. For the second configuration a rise in temperature of $\sim 109{ }^{\circ} \mathrm{C}$ is determined, with a dissipation time of $3.1 \mathrm{~ns}$. It is clear then, that the time for the samples to dissipate the heat energy from the pump laser pulses is much shorter than the arrival time of each pulsed laser being spaced 333 $\mu s$ apart. This means any annealing effects can be discounted in terms of eumelanin's excited state vibrational evolution. The peak shifting effects, and rapid vibrational decay observed in FSRS experiments also occur on much faster timescales, on the order of $p s$ and so such phenomena cannot describe our FSRS data.

\subsubsection{Temperature dependence of eumelanin's ground state Raman spectrum}

Temperature-dependent ground state Raman spectra are collected using a commercial spontaneous Raman spectrometer (Horiba Olympus BX411, Labram) configured with a $1800 \mathrm{gr} / \mathrm{mm}$ grating, $514 \mathrm{~nm} \mathrm{CW}$ laser, $600 \mathrm{~mm}$ objective lens and a $3 \mathrm{~s}$ integration time. A $150 \mathrm{~nm}$ thick eumelanin film was prepared and mounted in a purged cryostat (Linkam THMS600) and heated to $300{ }^{\circ} \mathrm{C}$ in $50{ }^{\circ} \mathrm{C}$ steps. The position of the film was not changed throughout the experiment with potential film degradation from exposure to the $\mathrm{CW}$ laser being tested by first increasing and then decreasing the temperature in $50{ }^{\circ} \mathrm{C}$ and $100{ }^{\circ} \mathrm{C}$ steps respectively, and comparing the data. 
The two main vibrational bands at $1590 \mathrm{~cm}^{-1}$ and $1390 \mathrm{~cm}^{-1}$ (referred to as the "G" and " $\mathrm{D}$ " bands hereafter for convenience) were analysed to determine their peak locations and intensity ratios as a function of temperature. Figure 6.15 shows the spectra collected, and the peak position of the $G$ and $D$ bands, as a function of temperature. Two features of interest are present in the data. The first is the change in the overall Raman intensity with increasing temperature, first increasing by $\sim 34 \%$ within a 150 ${ }^{\circ} \mathrm{C}$ rise, and then decreasing rapidly by $\sim 93 \%$ upon further increase from $150{ }^{\circ} \mathrm{C}$ to $300{ }^{\circ} \mathrm{C}$. Panel $b$ of the same figure shows the peak locations for the $\mathrm{G}$ and $\mathrm{D}$ bands as a function of increasing temperature. For the $\mathrm{G}$ band, an initial blue-shift is observed of $\sim 2 \mathrm{~cm}^{-1}$ from $50{ }^{\circ} \mathrm{C}$ to $150{ }^{\circ} \mathrm{C}$, and a subsequent red-shift of $\sim 5 \mathrm{~cm}^{-1}$ from 150 to $300{ }^{\circ} \mathrm{C}$. The high resolution of the system $\left(1 \mathrm{~cm}^{-1}\right.$ using a $1800 \mathrm{gr} / \mathrm{mm}$ grating) allows these small shifts to be captured with accuracy. For the D band, a larger red-shift of $25 \mathrm{~cm}^{-1}$ is observed at $100{ }^{\circ} \mathrm{C}$, followed by a return to the original peak position at 300 ${ }^{\circ} \mathrm{C}$. It can be concluded that these shifts in the ground state are dependent on heating, which may be due to the structural rearrangement of the molecule producing different vibrational modes than those present at room temperature.

Figure6.16 shows the peak intensities for the $G$ and $D$ bands as a function of temperature in panel $a$. Observed here is an initial rise in both intensities (consistent with the overall spectral intensity increase peaking at around $150{ }^{\circ} \mathrm{C}$ discussed above), with a clear trend towards decreasing intensity as temperatures rise to $300{ }^{\circ} \mathrm{C}$ Of particular interest is the intensity ratio of these two peaks, shown in panel $b$ of the same figure. Here we can see a clear increasing trend in the $G$ band intensity versus that of the $D$ band intensity, decreasing only at extremely high temperatures close to $300{ }^{\circ} \mathrm{C}$. The ratio of $\mathrm{G}$ and $\mathrm{D}$ bands also changes, as does the FWHM of each band - with the primary changes observed in the $\mathrm{G}$ band; blue-shifting and becoming narrower with increasing temperature. This is indicative of a temperature-dependent structural change in the material, which may explain in part eumelanin's success in funnelling the sun's energy away as it is heated.

The conditions used in these CW temperature dependent measurements are not comparable to those in FSRS experiments conducted using temporally short pulsed lasers. An approximation can be made between the two experiments, but is concluded here that these ground state temperature-dependent results reflecting broad peak shifts in the ground state that are not present in the FSRS data, which shows distinct localised excitation peaks with rapid $p s$ decay kinetics. 
a

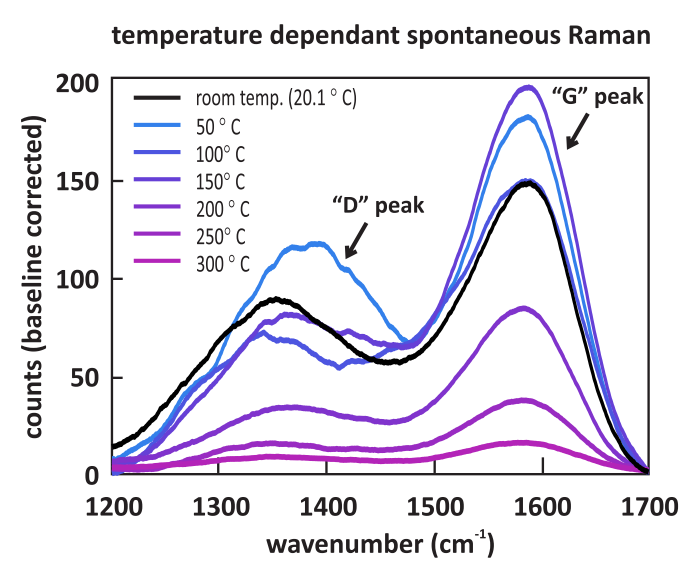

b

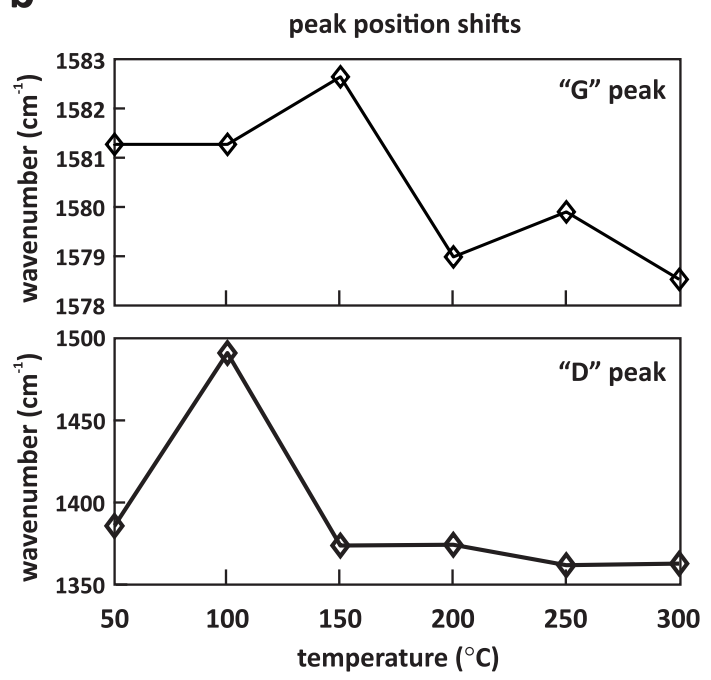

Figure 6.15: Panel $a$ shows spontaneous eumelanin spectra collected as a function of temperature, from room temperature to $300{ }^{\circ} \mathrm{C}$. Observations of the "G" and " $\mathrm{D}$ " like peaks can be seen to shift in spectral position as well as intensity. Panel $b$ tracks the peak position shifts for each peak. The graphs show a blue-shift peaking around $150{ }^{\circ} \mathrm{C}$ and $100^{\circ} \mathrm{C}$ for the $\mathrm{G}$ and $\mathrm{D}$ peak positions respectively.

\subsubsection{A study of eumelanin using time-resolved impulsive stimu- lated Raman spectroscopy}

A study of eumelanin films and dispersions using time-resolved impulsive stimulated Raman (TR-ISRS) spectroscopy is presented. TR-ISRS is a relatively recent spectroscopic tool able to interrogate the vibrational dynamics of complex materials with high spectral resolution across a broad spectral range $\left.{ }^{[168] 170}-178\right]$. Where TR-ISRS differs from that of FSRS, is primarily in the use of an ultra-short transform-limited Raman pump, rather than a temporally long, spectrally narrow-band Raman pump. The Raman pump, typically $\sim 7 f$ in duration, stimulates a vibrationally coherent wavepacket in the material, which then modulates the probe pulse in time. The subsequent "beating" of the probe pulse's intensity is recorded on a spectrograph, followed by Fourier transform to extract the spectral frequencies. The advantages of this technique over FSRS are numerous, including Gaussian shaped peaks devoid of dispersion, and devoid of baseline artefacts caused by cross phase modulation of the pump and probe 
a

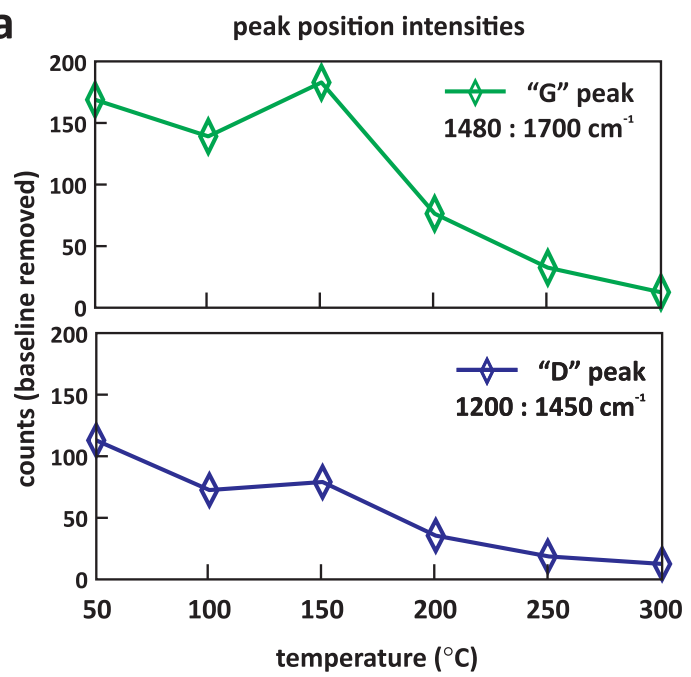

b

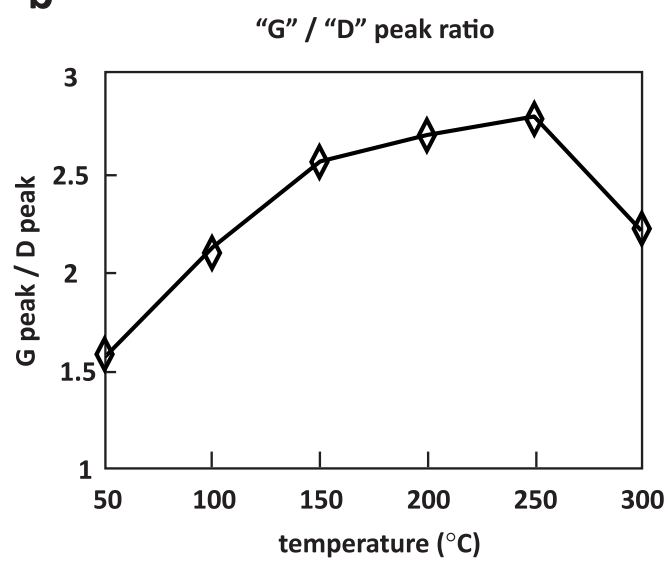

Figure 6.16: The " $\mathrm{G}$ " like " $\mathrm{D}$ " like peak position intensities are shown in panel $a$ as a function of temperature. Here we see an increase in both peak intensities around $150^{\circ} \mathrm{C}$, followed by a decline. Panel $b$ shows the ratio of the " $\mathrm{G}$ " to " $\mathrm{D}$ " like bands, increasing with temperature to $\sim 250{ }^{\circ} \mathrm{C}$. This trend could be explained by a structural re-arrangement of the molecule, presenting as an increase in phonons which are prevalent in the more ordered arrangement of the graphitic " $G$ " band

pulses, both present in FSRS. The details of the TR-ISRS method are described in detail elsewhere. In collaboration with Riken Laboratories in Wako-shi, Japan, a TR-ISRS study was undertaken on eumelanin films and dispersions for comparison with FSRS experiments performed at the Victoria University of Wellington.

The experimental set-up for these measurements involves sample preparation of eumelanin films and dispersions in the same method described in section 6.3.2. The excitation and Raman pumps are $400 \mathrm{~nm}$ pulses of $\sim 7 f_{s}$ duration. The broadband probe spans the UV and IR regions, and is generated in the same method as the probe pulses in the FSRS technique, described in section 4.1. Back scatter from the $1 \mathrm{~mm}$ film substrate limits the temporal range available to $\sim 100 \mathrm{ps}$ as interference from the secondary scattered pulse contaminates the recorded probe signatures after this time. In the case of the eumelanin dispersion, a longer temporal range is achieved using a 3 $m m$ cuvette. A flow cell is used at a rate of $\sim 10 \mathrm{~mL} /$ minute to continually purge the sample, ensuring no build up of the excited state population in the beam path. 

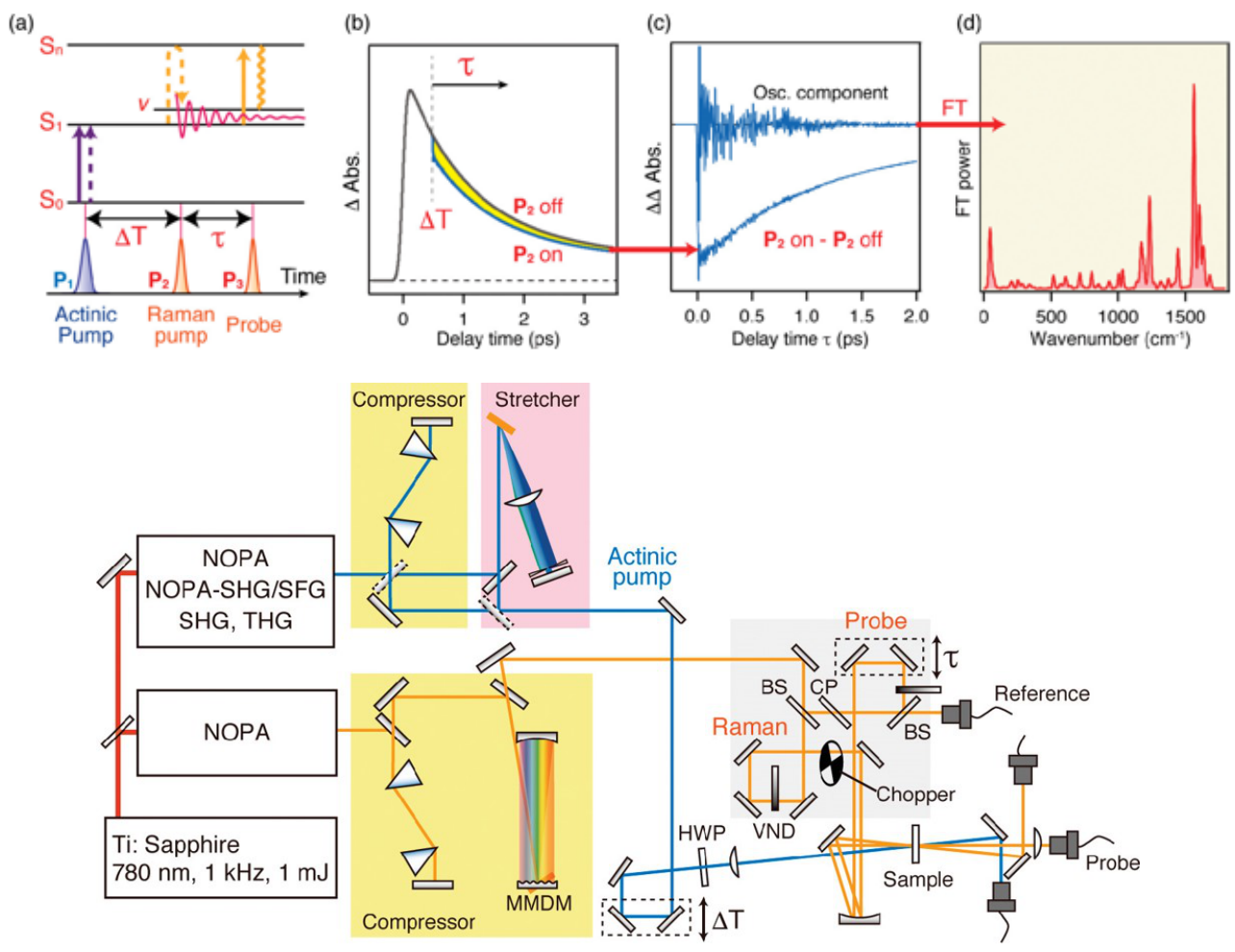

Figure 6.17: Figure (a) of the upper panel shows a schematic diagram representing the optical transitions involved in the TR-ISRS experiment. ((b)-(d)) Schematic pictures representing the signal analysis procedure of the TR-ISRS experiment. A differential signal of the pump-probe data between with and without the Raman pump pulse (b) provides the raw TR-ISRS signal (c). A frequency-domain vibrational spectrum (d) is obtained by Fourier transformation of the oscillatory component of the raw TR-ISRS data. The lower panel shows a schematic diagram of the TR-ISRS setup. NOPA: noncollinear optical parametric amplifier; MMDM: micromachined membrane deformable mirror; HWP: half wave plate; BS: beam splitter; CP: compensation plate; VND: variable neutral density filter. All the optics in the gray shaded area are mounted on a 59-mm height breadboard. Thes figures are reproduced from the publication: Kuramochi, Hikaru, Satoshi Takeuchi, and Tahei Tahara. "Femtosecond time-resolved impulsive stimulated Raman spectroscopy using sub-7-fs pulses: apparatus and applications." Review of Scientific Instruments 87.4 (2016): 043107. [168], with the permission of AIP Publishing.

Results of the TR-ISRS experiments are presented in figures 6.18 and 6.19 for eumelanin film and dispersion. The broadband absorption of eumelanin unfortunately leads to ground state contamination of the extracted spectral energies, with the Raman peaks at 

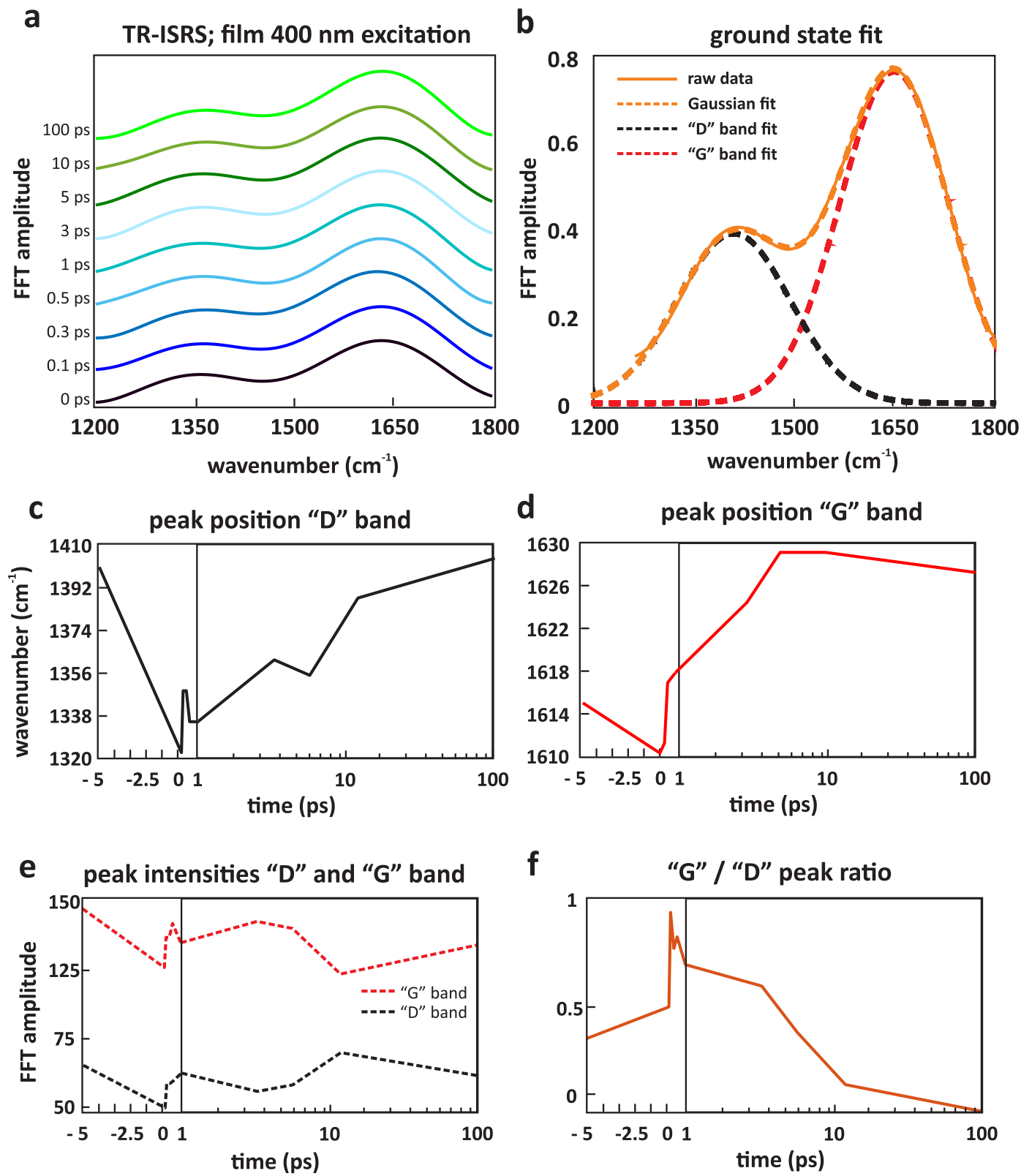

Figure 6.18: TR-ISRS data is presented here for eumelanin films at $400 \mathrm{~nm}$ excitation, and a $400 \mathrm{~nm}$ Raman pump. Spectral data recovered from Fourier transform of the probe's intensity is shown in panel $a$, with a similar broad ban structure as observed in eumelanin's ground state. Trends in the peak positions of the D and $\mathrm{G}$ like bands can be observed, as well as their intensity ratios. These results are shown in panels $c$ - $f$ - with the Gaussian deconvolution function used to analyse this data presented in panel $b$. These figures are presented with the permission of the Riken Laboratories of Wako-shi Japan led by Professor Tahei Tahara ${ }^{[169]}$. 

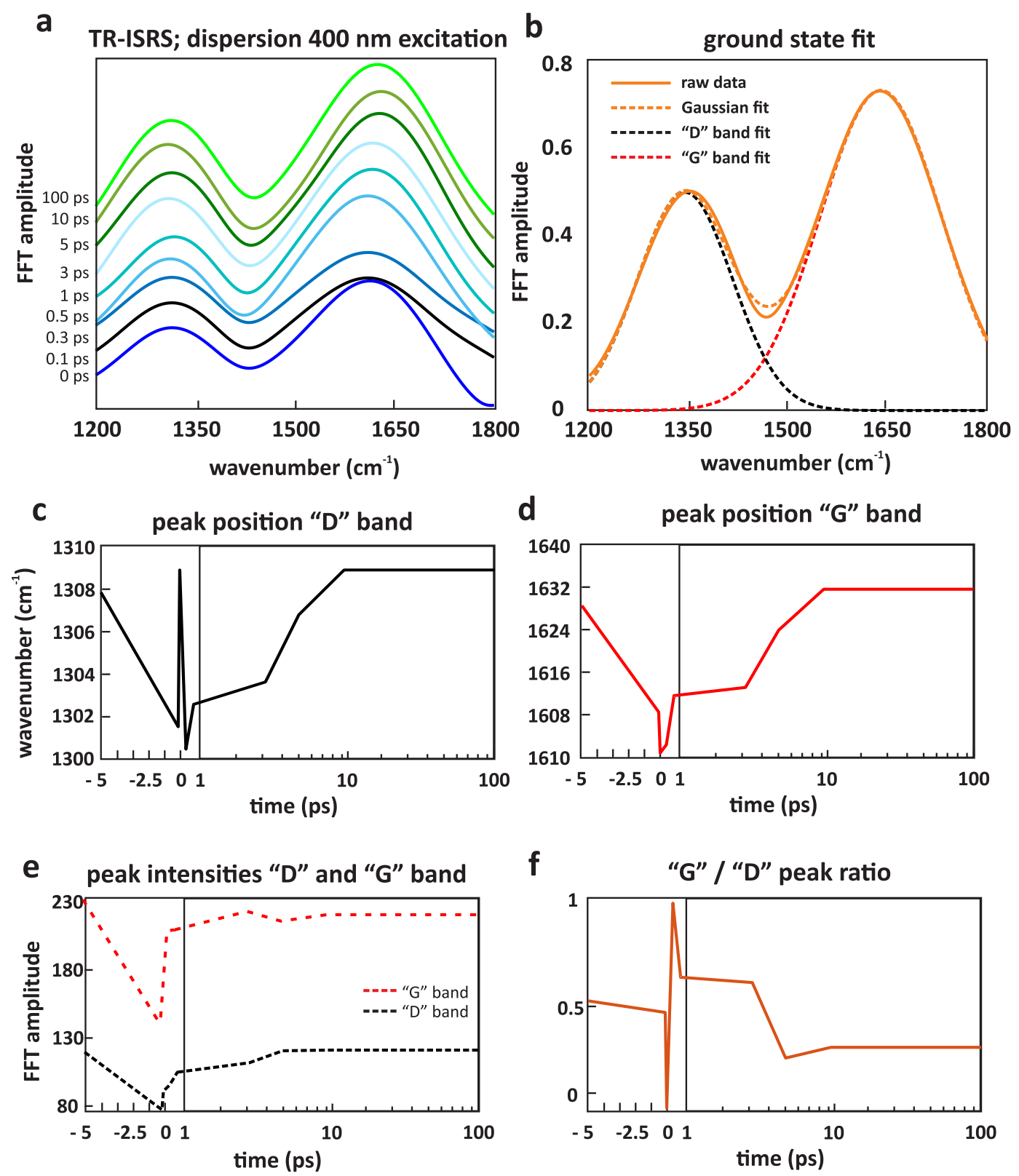

Figure 6.19: As previously detailed in figure 6.18. TR-ISRS data is presented here for eumelanin dispersions at $400 \mathrm{~nm}$ excitation, and a $400 \mathrm{~nm}$ Raman pump. A similar trend in the shifting of Raman peak positions with time is observed, as is the rapid rise and decay of the G/D peak ratio. These figures are presented with the permission of the Riken Laboratories of Wako-shi Japan led by Professor Tahei Tahara 169 .

1350 and $1590 \mathrm{~cm}^{-1}$ remaining present in the data upon Fourier transform. This is due to absorption of the visible $400 \mathrm{~nm}$ Raman pump by the material, and it is noted that 
future experiments using this method would see the Raman pump ideally tuned further into the IR, where eumelanin has significantly lower absorption rates. While the exact excited state spectral components within this envelope cannot be isolated from ground state absorption, dynamic changes within these broadband peaks still provide useful information of eumelanin's excited state energy dissipation by observation of peak intensity ratios and spectral shifts. This study has provided insights into eumelanin's ground state thermal behaviour, and has also confirmed FSRS as the correct tool for the direct observation of eumelanin's excited state relaxation mechanism. The frequency domain approach is favourable to isolate the vibrational mechanisms of the individually excited monomer and oligomer species.

\subsection{Chapter summary}

We have shown in this research the first evidence for localised vibrational excitations in eumelanin pigment upon UV and visible light excitation. Mapping the DHICA vibrational spectral signatures onto the larger macro-molecule provides further evidence of excited state proton transfer (ESPT) as eumelanin's key non-radiative dissipation mechanism, resolved on $f s$ - ps timescales. The structural characterisation and vibrational mode assignment of eumelanin is challenging given the highly disordered nature of the material, reflected in its broad band ground state Raman spectrum. Here, using FSRS, we are a step closer to uncovering the true nature of eumelanin's excited state non-radiative vibrational dissipation pathways. By the observation of eumelanin's excited state vibrational dynamics, we see a clear link to those of its DHICA subunit. In DHICA, ESPT is directly observed as presented in chapter 5, and mapping these spectral signatures onto the eumelanin macro-molecule strongly suggests the same mechanism is occurring. The study of eumelanin's DHI monomer in terms of ESPT is inherently more challenging given that DHI in its oxidised state rapidly polymerises. A complete picture of how the energy dissipation mechanisms of eumelanin's monomers and oligomers contribute to the macro-molecule's relaxation pathways therefore requires further studies.

Observing the FSRS signatures of DHICA in ammonia (used in eumelanin film preparations) is useful for future work - here the DHICA monomer will not de-protonate upon solvation, so that the ESPT of its phenol groups may not be the favourable relaxation pathway. The analysis of the excited state vibrational dynamics for dispersions of 
eumelanin in ammonia have been presented here, however it is not possible to directly compare these with with its DHI and DHICA subunits dissolved in water, due to their solvent dependence. Certainly, the large and disordered eumelanin macro-molecule will not have fully de-protonated under such conditions, and so a comparison will not be conclusive without future investigation.

An additional hypothesis presented is that of excitations being deactivated via localised states. Evidence for this hypothesis is given by the comparison of eumelanin's ground state optical behaviour (in it's absorption, emission, Raman spectrum and resonance Raman behaviour) with that of its excited state Raman signatures, as well as its fluence independent TA spectra.

Heating effects on eumelanin have been presented, and provide interesting insights into its ground state vibrational behaviour. One of eumelanin's primary functions in Nature is to absorb sunlight and disperse photon energies, as heat, on fast timescales before damage can occur to underlying cells in human skin. Eumelanin's response to heating must therefore be an inherent property of the material. It is challenging to reference this via experimentation. Temperature dependent experiments conducted using a continuous wave laser show a definitive heat dependence of the main ground state peak positions and intensity ratios, supported by TR-ISRS studies. This can also be compared with similar studies performed on amorphous carbons summarised in this chapter. These experiments cannot be compared directly in terms of excited state dynamics as each $f s$ laser pulse exists only briefly in the material.

Finally, we can present this research as an exciting step forward in validating previous studies into eumelanin and its subunit structures, in terms of their photophysical energy dissipation mechanisms. Transient absorption studies, along with UV-Vis absorption spectra, give strong indications of distinct overlapping chromophore building blocks making up the eumelanin macro-molecule. Here, using FSRS, we can now support this hypothesis by presenting direct evidence of ESPT in the DHICA subunit, and localised vibrational energies being selectively excited upon photo-excitation of the eumelanin macro-molecule, and relaxing on the same rapid $p s$ time scales. 


\section{Chapter 7}

\section{Research conclusions and outlook}

In this research project we aim to reveal the photophysical processes contributing to the unique UV-thermal vibrational dissipation mechanism of eumelanin pigment.

This thesis is set out to answer the following questions:

- What photophysical processes contribute to eumelanin's UV non-radiative energy dissipation mechanism?

- What are the key modes of vibrational relaxation and how are they linked together?

- Is extreme structural disorder an essential or contributing component of the nonradiative energy dissipation mechanism?

- Does ESPT occur in the natural eumelanin pigment, and its DHI and DHICA subunit building blocks, as a contributor to the photo-protection mechanism?

By applying the spectroscopic technique of FSRS to this unique and elusive material we have demonstrated the first direct evidence of ESPT in eumelanin's DHICA subunit, and used these vibrational signatures to directly map the eumelanin macro-molecule's vibrational excited state. Evidence is clear that while the steady state Raman signatures of eumelanin present as overlapping bands comprising of many spectral species, isolated chromophores are selectively photo-excited upon light irradiation. Observing eumelanin in its condensed phase, and in dispersions, shows interesting comparisons. Additionally, solvent dependant studies of its DHICA subunit show significant differences in the decay of each excited vibrational mode, lending further evidence for its ESPT deactivation mechanism. 
As outlined in chapter 4 , the first stage of this project was to adapt the current TA system under operation in the ultrafast laser spectroscopy group of the Victoria University of Wellington to an FSRS system with the addition of a Raman pump laser beam and a high-resolution transmission grating spectrograph. A significant amount of time spent in this research project was on this optical build, including all the stages of system design, build, testing and improvements. Two Raman pump experiment configurations were designed and built to generate Raman pulses in the visible and IR regions as described in chapter 3. This tunability is important in terms of achieving resonance enhancement with the PIA bands of eumelanin and its monomers and both systems were used accordingly. For each different Raman pump wavelength, a separate spectrometer was built to capture the Stokes' shifted region with respect to the Raman pump wavelength, one in the visible and the second in the IR. Two different detectors and optical set ups were required to achieve this, with the system designs centred around achieving suitable optical resolution based on the detector details and optical elements. Adjustments to shot referencing were also required when switching between the two configurations, where the detector used in the visible spectrometer required the illumination of a pixel while the PDA detector used in the IR had a separate photo-diode reference. Two excitation pump wavelengths were used in the FSRS experiments, requiring the design and build of a second and third harmonic generation system for pup pules in the UV $(267 \mathrm{~nm})$ and visible $(400 \mathrm{~nm})$. The UV pulses generated were useful in the comparison of the behaviour of eumelanin to its monomers, as DHI and DHICA absorb in the UV, but not at $400 \mathrm{~nm}$. The visible excitation pump was used therefore in the comparison of eumelanin when absorbing different wavelengths.

The post-processing data analysis software also contributed to a significant amount of research time in this project, with additional software written to extract and model the dynamic Raman spectra. The collected data must be loaded, averaged and sequenced correctly, with the additional processing steps required to extract the FSRS surfaces, including calibration, baseline removal and solvent normalisation, being automated as much as possible.

With the system build completed, the study of eumelanin's subunits in terms of the vibrational signatures shows interesting comparisons of the DHI and DHICA monomers, as well as a solvent dependant study of DHICA, described in chapter 5. While the DHI monomer proved challenging to gather robust data due to it's rapid polymerisation in air and upon light exposure, some insights can still be made even in a polymerised form. Experimenting on DHICA itself provided fascinating direct evidence of ESPT 
when compared with DFT analysis of DHICA's anion and di-anion structures. Future studies of the DHI and DHICA subunits dissolved in varying $\mathrm{pH}$ solvents may provide interesting insights into this mechanism, where restrictions on the initial deprotonation of the materials to the solvent may show other preferable non-radiative energy dissipation pathways.

The direct studies on eumelanin pigment in chapter 6 give fascinating insights into its ground and excited state behaviours, with strong evidence of excitations decaying via localised states presented in the TA and Raman data, despite its broad band spectral signatures in the ground state. When comparing the FSRS surfaces of eumelanin in films and dispersions we see that the vibrational modes in films appear to shift in frequency throughout the dephasing time, similarly to those observed in DHICA where ESPT is observed. These shifts are present for both excitation pump wavelengths in the film data, but not observed for either in the dispersion data - lending further evidence of an ESPT deactivation pathway in the eumelanin molecule that is not available when prepared in an ammonia dispersion. Further studies and investigations of eumelanin give addition insights, such as its resonance Raman behaviour, frequency peak dependence on temperature and the comparison of the Raman signatures to that of disordered carbon.

Studying eumelanin is challenging given its highly disordered nature, and an apparent refusal to give up Nature's "secrets" via optical interrogation, an irony given that eumelanin's main function is believed to be photo-protection. Has Nature found a way in this pigment to protect us from the sun via random chemical disorder, or was it purposefully made to be this way in a concise structural arrangement?

Future work may involve FSRS studies of eumelanin and its subunits over a wider excitation pump wavelength range, particularly towards the deep UV. Here we may see a wavelength-dependent trends occurring across a wider wavelength range in terms of the excitations of individual monomer and oligomer species, the building block of eumelanin, as well as of the pigment itself. Applying a variety of ultrafast spectroscopic studies to eumelanin and its constituents in a variety of solvents (especially at different $\mathrm{pH}$ levels) and phases will also build a broader picture of the energy dissipation mechanisms, both radiative and non-radiative, with the ultimate goal being a global model of eumelanin's structure and photophysical behaviour. Future improvements to the FSRS system built during this project include optimisation of the bandwidth compressor to generate usable Raman pumps across a broader spectral range, especially 
towards the IR where PIA bands are common in organic materials, without the need for a secondary spatial filtering system. Further automation of the post-processing software is also desirable, for example bulk loading of pump power data files, spot size data and calibration files.

In this project we believe that FSRS has provided a first step towards the goal of revealing eumelanin's photo-protection mechanism pathways. Here we have resolved excited state vibrational kinetics as a function of excitation wavelength for eumelanin, and additionally for its subunit monomers DHI and DHICA. Using the FSRS data from this research in collaboration with further ultrafast spectroscopic experiments, future work may reveal complete dynamic structural information of eumelanin's UVthermal energy transfer system, revealing deep insight Nature. Looking further on, this research may also contribute to potential biomimetic frameworks for building new, highly-efficient and photo-stable UV absorbing materials. 


\section{Appendix A}

\section{FSRS system build layout - technical drawings}

A.1 Complete FSRS optical system

A.2 Raman pump generation - bandwidth compressor (grating configuration)

A.3 Excitation pump generation - second and third harmonic generation

A.4 Sample area and spectrometer - $400 \mathrm{~nm}$ Raman pump configuration 


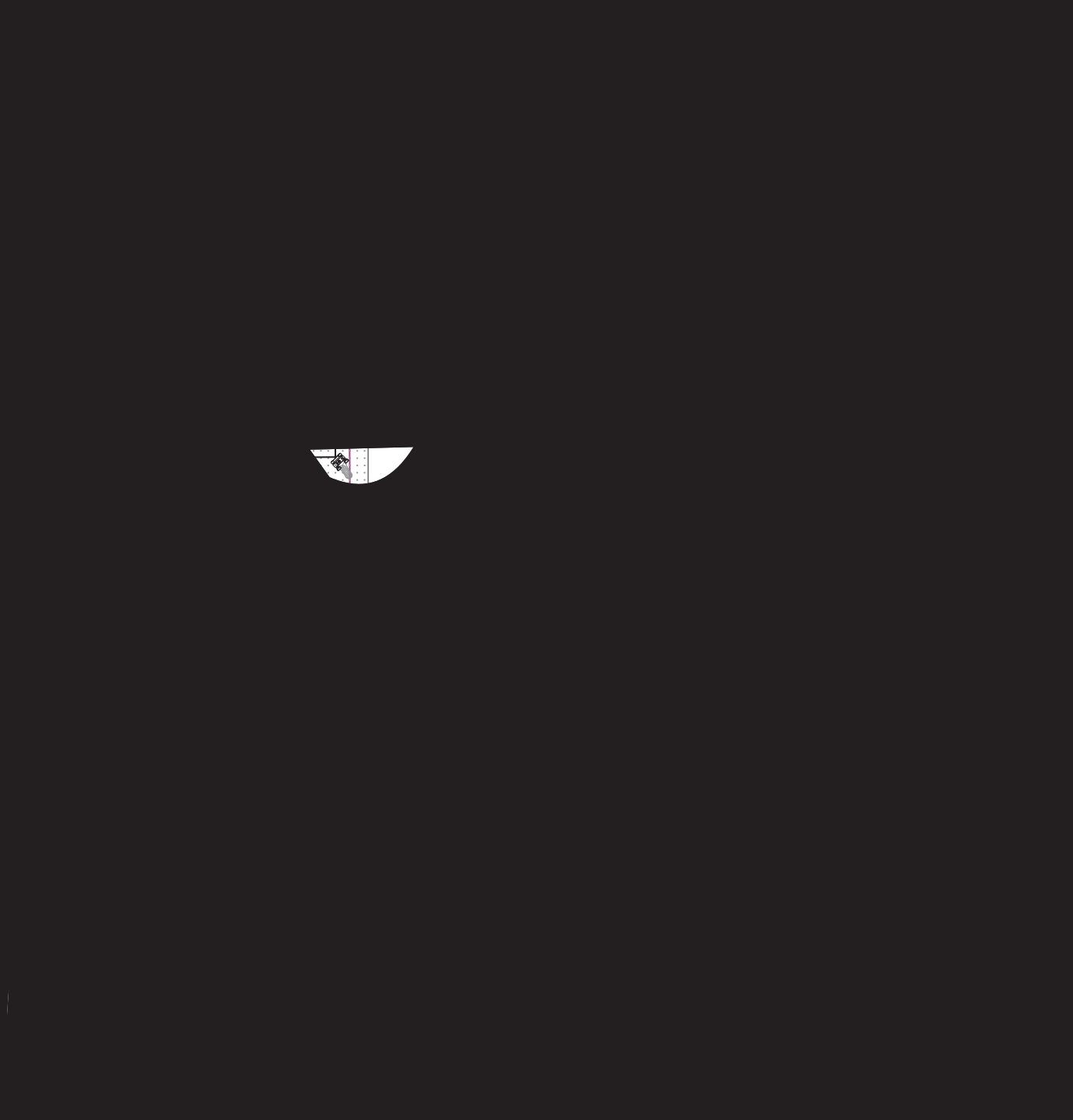

Figure A.1: The technical drawings of the optical system are generated using CorrelDRAW X7 (64-bit) software. This figure shows the complete layout of the FSRS system, including the laser source, in a true representation of the Ultrafast spectroscopy lab tables at the Victoria University of Wellington. It should be noted that several other working systems co-exist with the FSRS system in our lab, meaning the physical layout of the FSRS system had space restrictions and was planned with this in mind. The key areas are indicated in text, that is - the Ti:Sapphire amplifier system; the THG and SHG actinic pump generation arm, the time delay line via an automated delay stage; pathlength compensation via a manual delay stage; probe generation; bandwidth compression; the sample area; and, lastly, the three spectrometers used to capture the relevant spectra: Raman spectrometer 1 (400 - 450 $\mathrm{nm})$, TA and Raman 2 (800 - $1100 \mathrm{~nm})$. 


\section{Bandwidth compressor (grating configuration)}

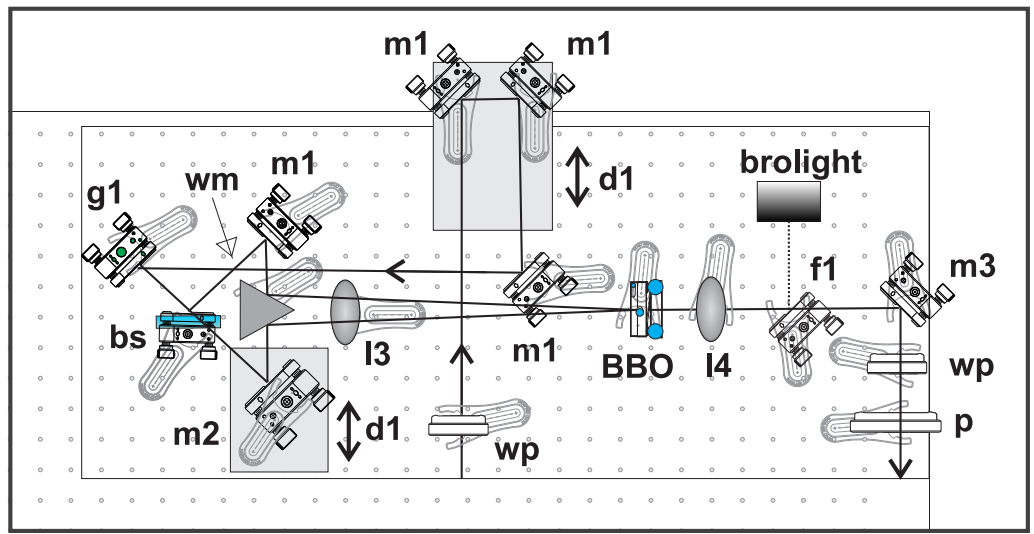

m1 plano silver mirror

wp wave plate

d1 manual delay stage

g1 $1200 \mathrm{gr} / \mathrm{mm}$ gold grating

bs $50: 50$ beam splitter

m2 2" plano silver mirror

wm plano silver wedge mirror
I3 achromatic doublet $\mathrm{f}=25 \mathrm{~mm}$

BBO type-1 BBO crystal I $=0.5 \mathrm{~mm}$

I4 cylindrical lens $\mathrm{f}=5 \mathrm{~mm}$

f1 flipping mounted plano mirror

m3 plano aluminium mirror

p wire-grid polariser

Figure A.2: The technical drawing of the bandwidth compressor (in the grating configuration) is presented. The optical table has screw holes spaced $2.5 \mathrm{~cm}$ apart. Each component is described in terms of their key properties, however specific optical brands and part numbers are excluded. While the Raman pump was generated in a variety of test configurations, this design proved to be the most robust in terms of ease-of-use, and optimal spectral compression and power conversion. It should be noted that the fundamental laser pulse is mechanically chopped at 1.5 $k H z$ before being sent into the bandwidth compressor. 


\section{Excitation pump: second \& third generation and delay stage}

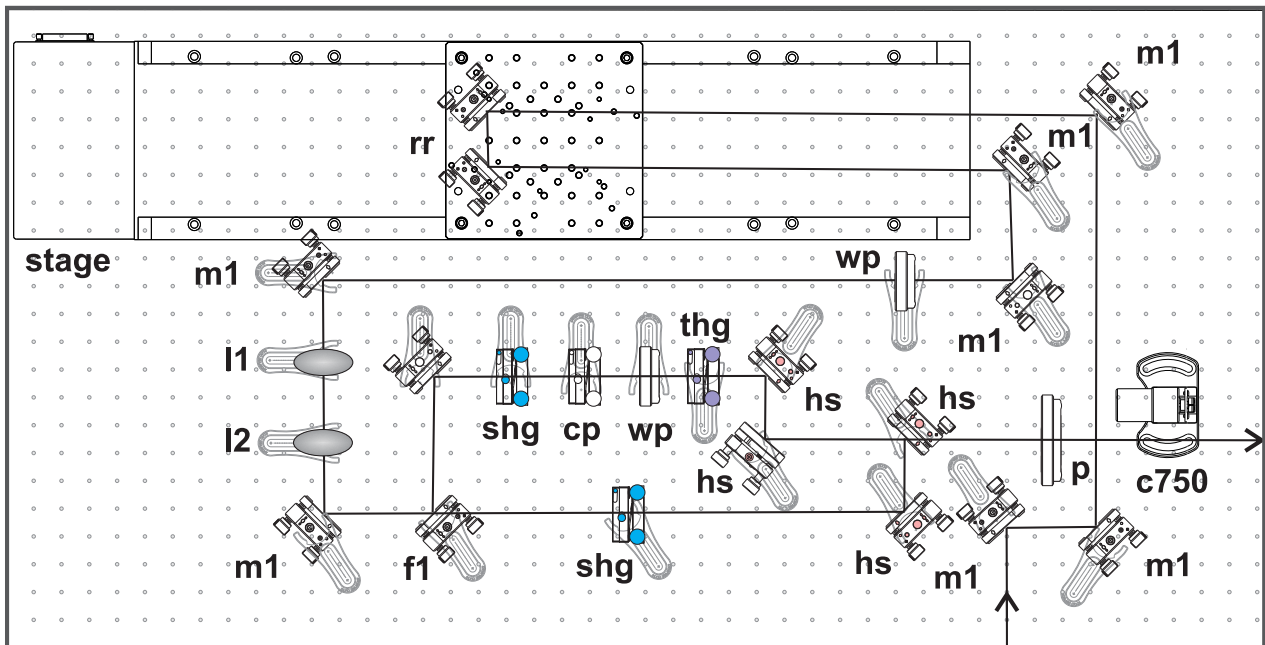

$\begin{aligned} \text { m1 } & \text { plano silver mirror } \\ \text { rr } & \text { retro-reflector } \\ \text { stage } & \text { plano silver mirror } \\ \text { wp } & \text { wave plate } \\ \text { I1 } & \text { convex lens } \mathrm{f}=9 \mathrm{~mm} \\ \text { I2 } & \text { concave } \mathrm{f}=-3 \mathrm{~mm} \\ \text { f1 } & \text { plano silver mirror (flipper mount) }\end{aligned}$

$$
\begin{aligned}
& \text { shg } \text { second harmonic crystal } \\
& \text { cp } \text { calcite compensation plate } \\
& \text { thg } \text { third harmonic crystal } \\
& \text { hs } \text { harmonic separator } \\
& \text { p } \text { wire-grid polariser } \\
& \text { c750 mechanical chopper } 750 \mathrm{~Hz}
\end{aligned}
$$

Figure A.3: The technical layout of the second and third harmonic generation system, as well as the delay line is presented. The retro-reflector is mounted on an electronic delay stage allowing time step precision of $\sim 100 \mathrm{fs}$ across a $n s$ time range. The second and third harmonic generation allows quick conversion between the generation of an excitation pump at $400 \mathrm{~nm}$ and $267 \mathrm{~nm}$ respectively. The excitation is chopped at $750 \mathrm{~Hz}$ using a mechanical chopper before being sent to the sample area. 
Sample area and spectrometer (400 $\mathrm{nm}$ Raman pump)

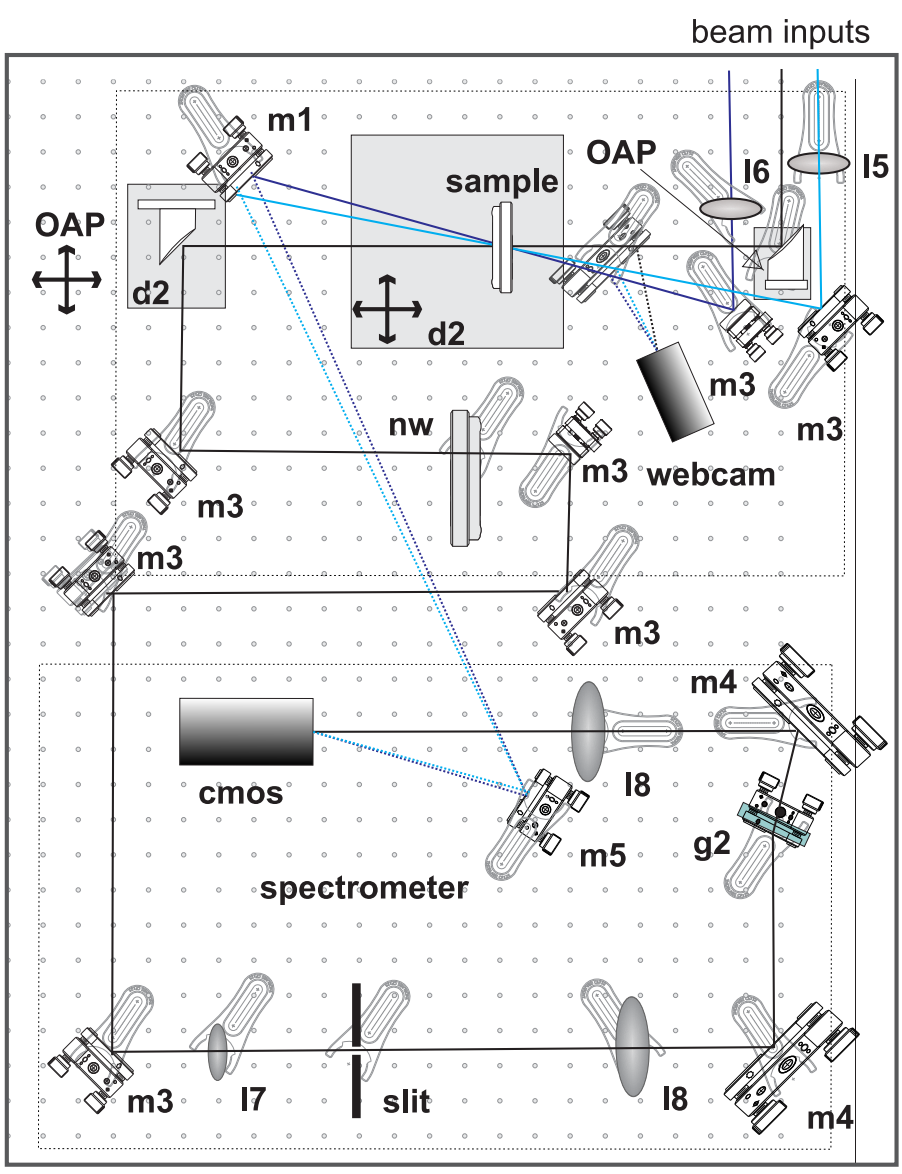

\begin{tabular}{|c|c|}
\hline & $\begin{array}{l}\text { excitation pump } \\
\text { Raman pump }\end{array}$ \\
\hline m1 & plano silver mirror \\
\hline m3 & plano Al mirror \\
\hline m4 & $\begin{array}{l}\text { concave mirror } \\
\mathrm{f}=15 \mathrm{~mm}\end{array}$ \\
\hline 15 & 2" plano Al mirror \\
\hline m5 & $\begin{array}{l}\text { UV coated lens } \\
f=27 \mathrm{~mm}\end{array}$ \\
\hline 16 & $\begin{array}{l}\text { UV coated lens } \\
f=25 \mathrm{~mm}\end{array}$ \\
\hline 17 & $\begin{array}{l}\text { UV coated lens } \\
\mathrm{f}=10 \mathrm{~mm}\end{array}$ \\
\hline 18 & $\begin{array}{l}\text { UV coated lens } \\
\mathrm{f}=250 \mathrm{~mm}\end{array}$ \\
\hline OAP & $\begin{array}{l}\text { off axis parabolic mirror } \\
\text { efl }=20.1 \mathrm{~mm}\end{array}$ \\
\hline d2 & $x-y-z$ manual stage \\
\hline nw & notch filter wheel \\
\hline g2 & $\begin{array}{l}\text { transmission grating } \\
600 \mathrm{gr} / \mathrm{mm}\end{array}$ \\
\hline
\end{tabular}

Figure A.4: The technical layout of the sample area and spectrometer used to collect the probe (and reference pump beams) is presented. This configuration is representative of the optics required for a UV excitation pump and a $400 \mathrm{~nm}$ Raman pump generated using the bandwidth compressor in the grating configuration as detailed in figure A.2 of this appendix. The mirror coatings are of particular importance when using UV light, with aluminium (Al) being preferred to silver coated mirrors. Similarly, the transmissive optics are chosen for maximum transmission in the UV. 


\section{Appendix B}

\section{Matlab code used for FSRS data post-processing}

\section{B.1 Chopper referencing for the CMOS detector}

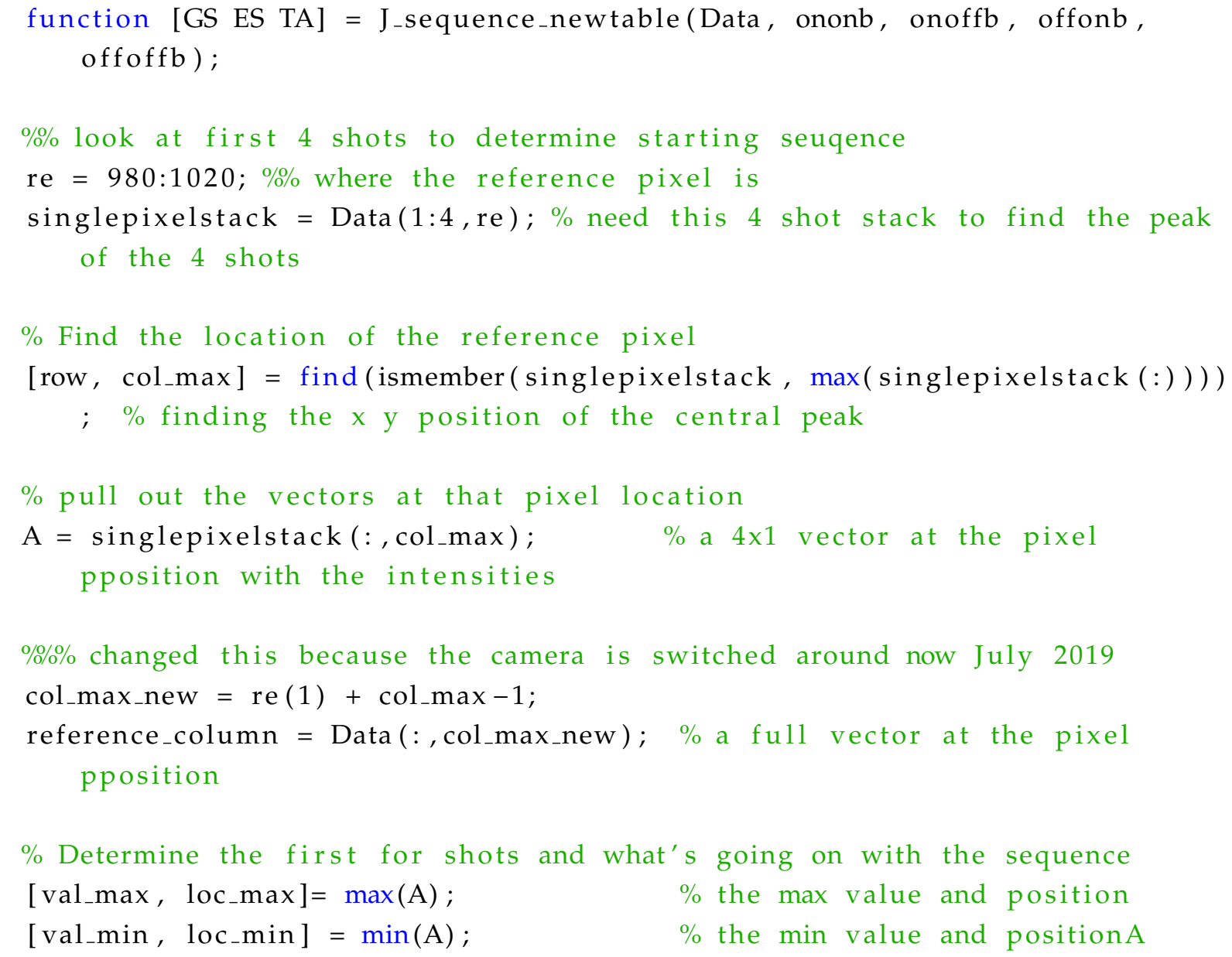




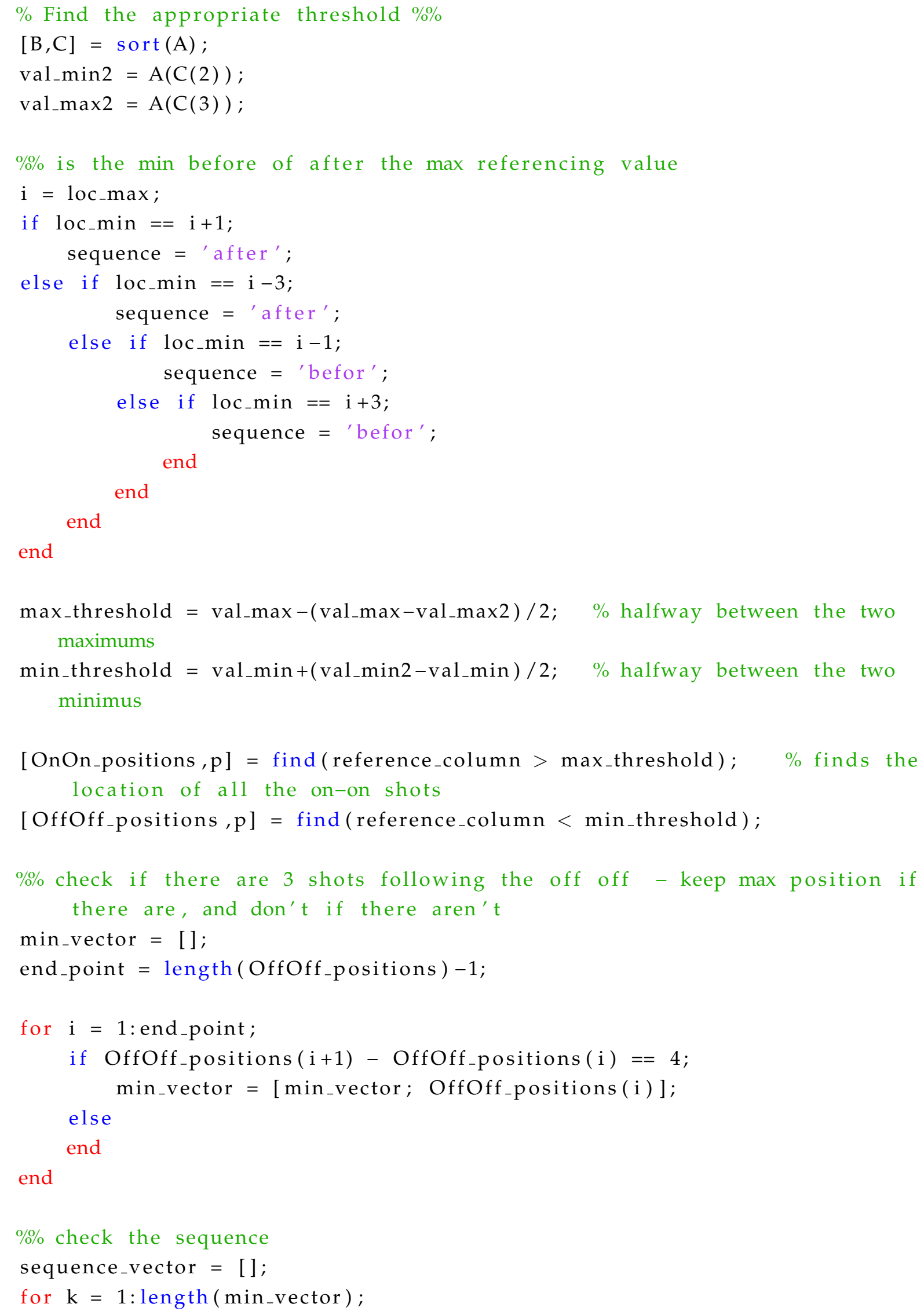




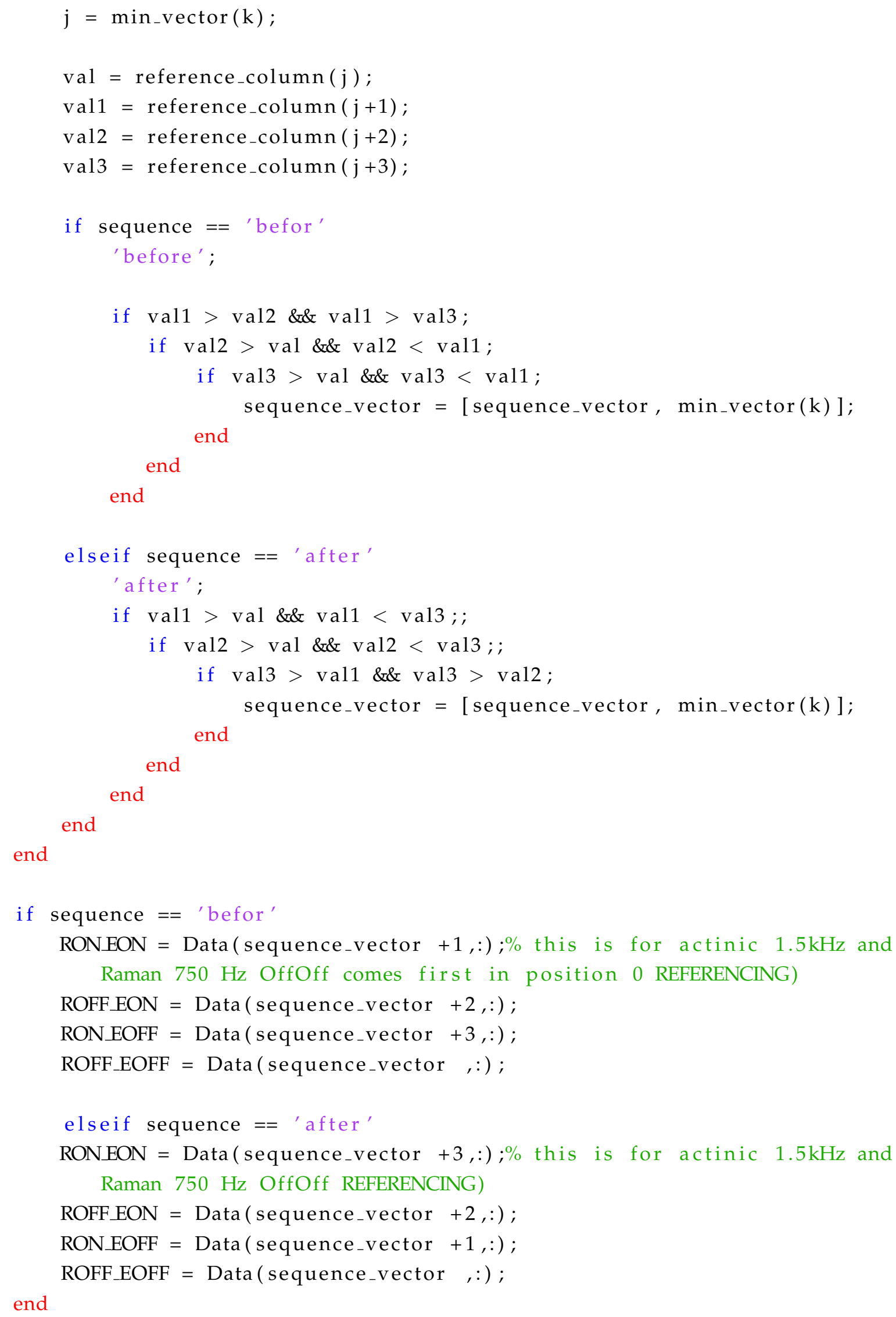




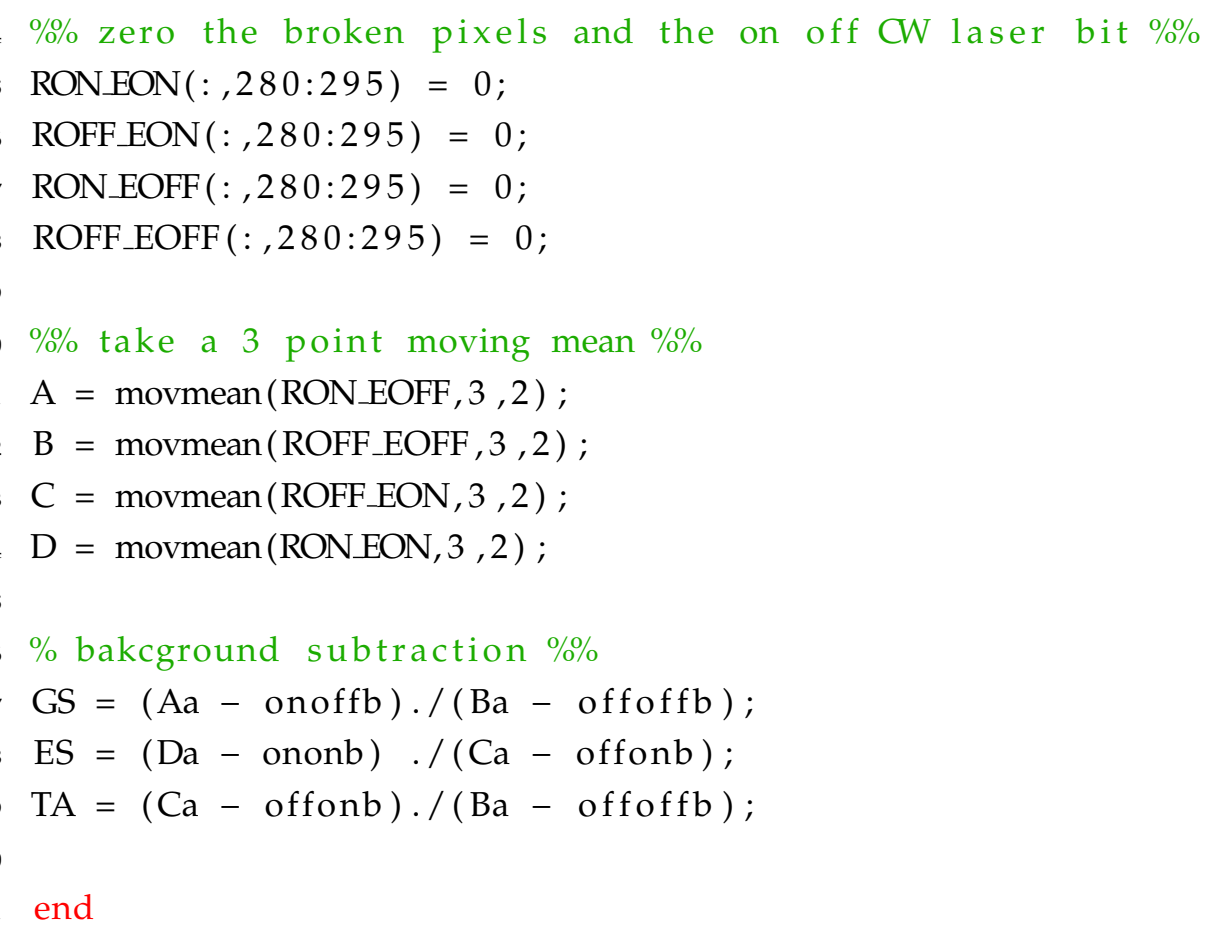

\section{B.2 Chopper referencing for the PDA detector}

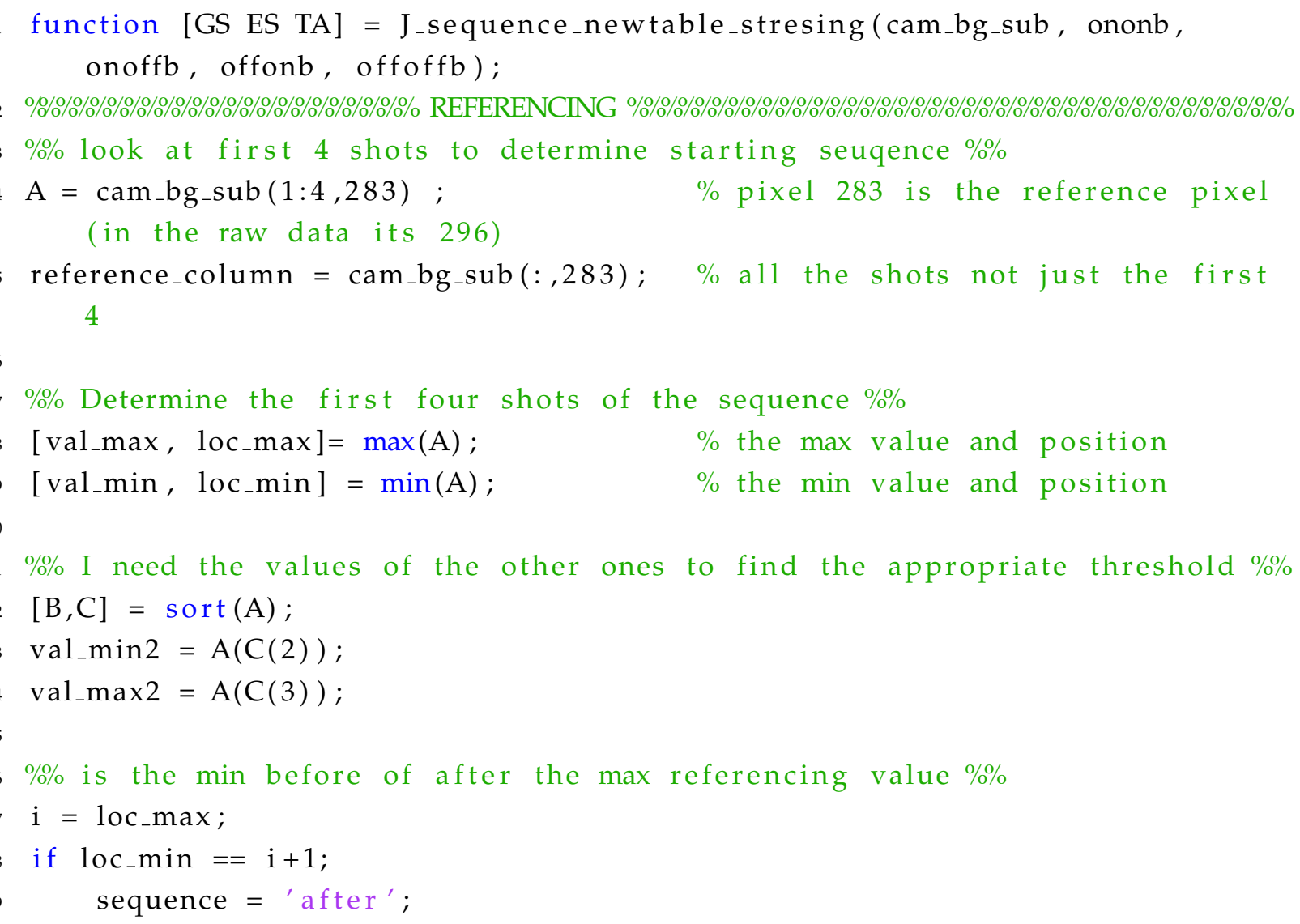

Page 156 


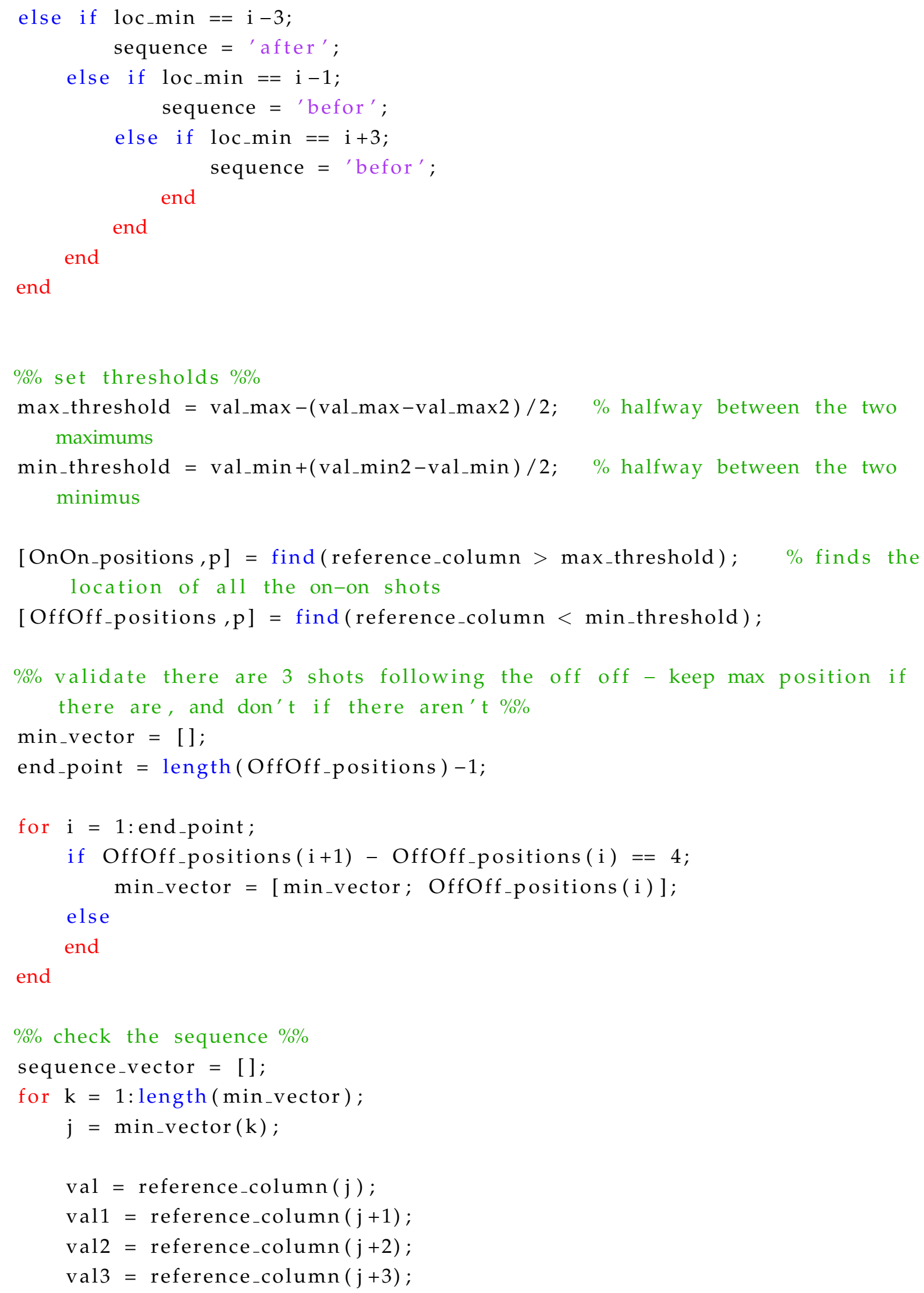




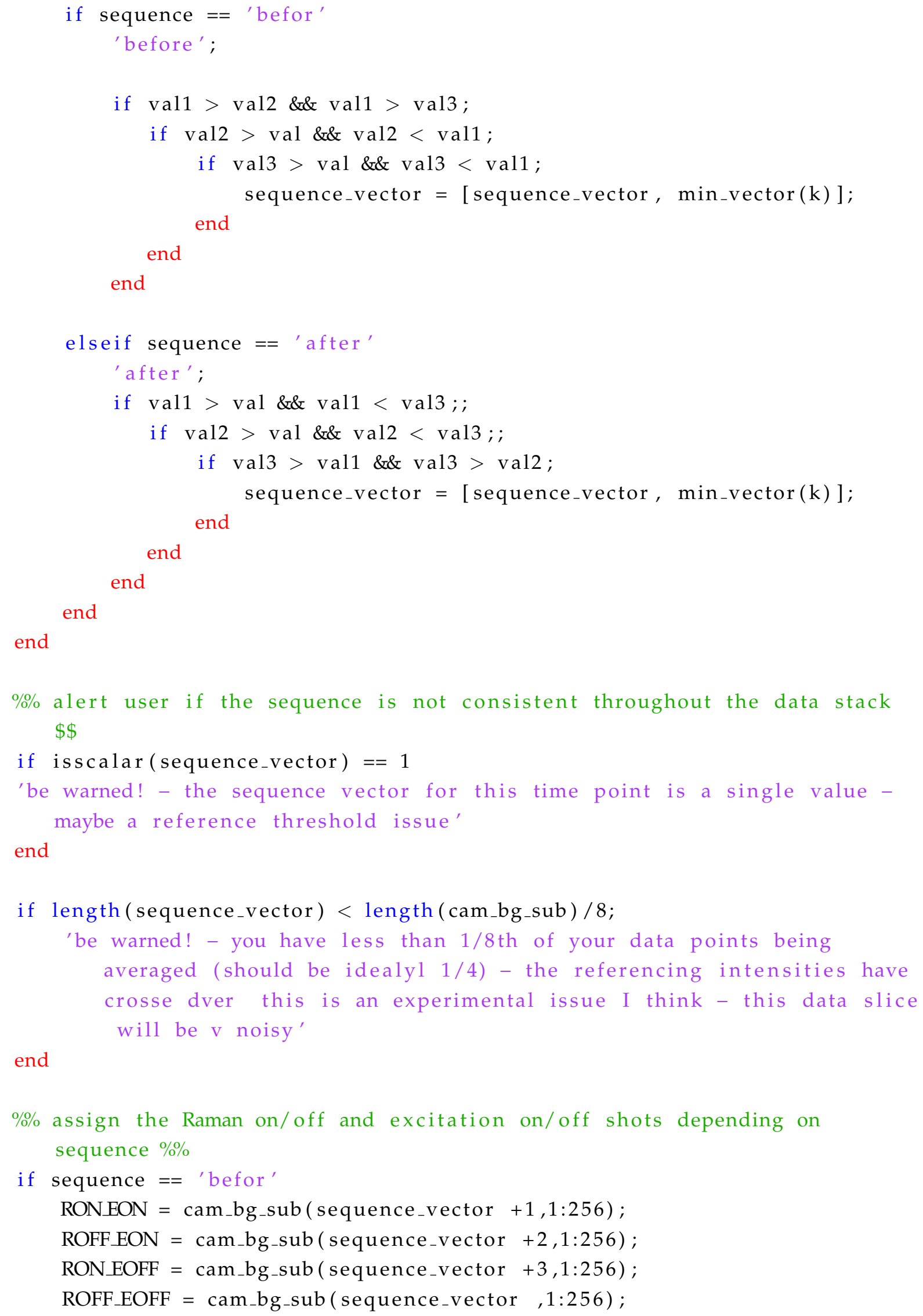




\section{B.3 Calibration of the CMOS detector using a standard}

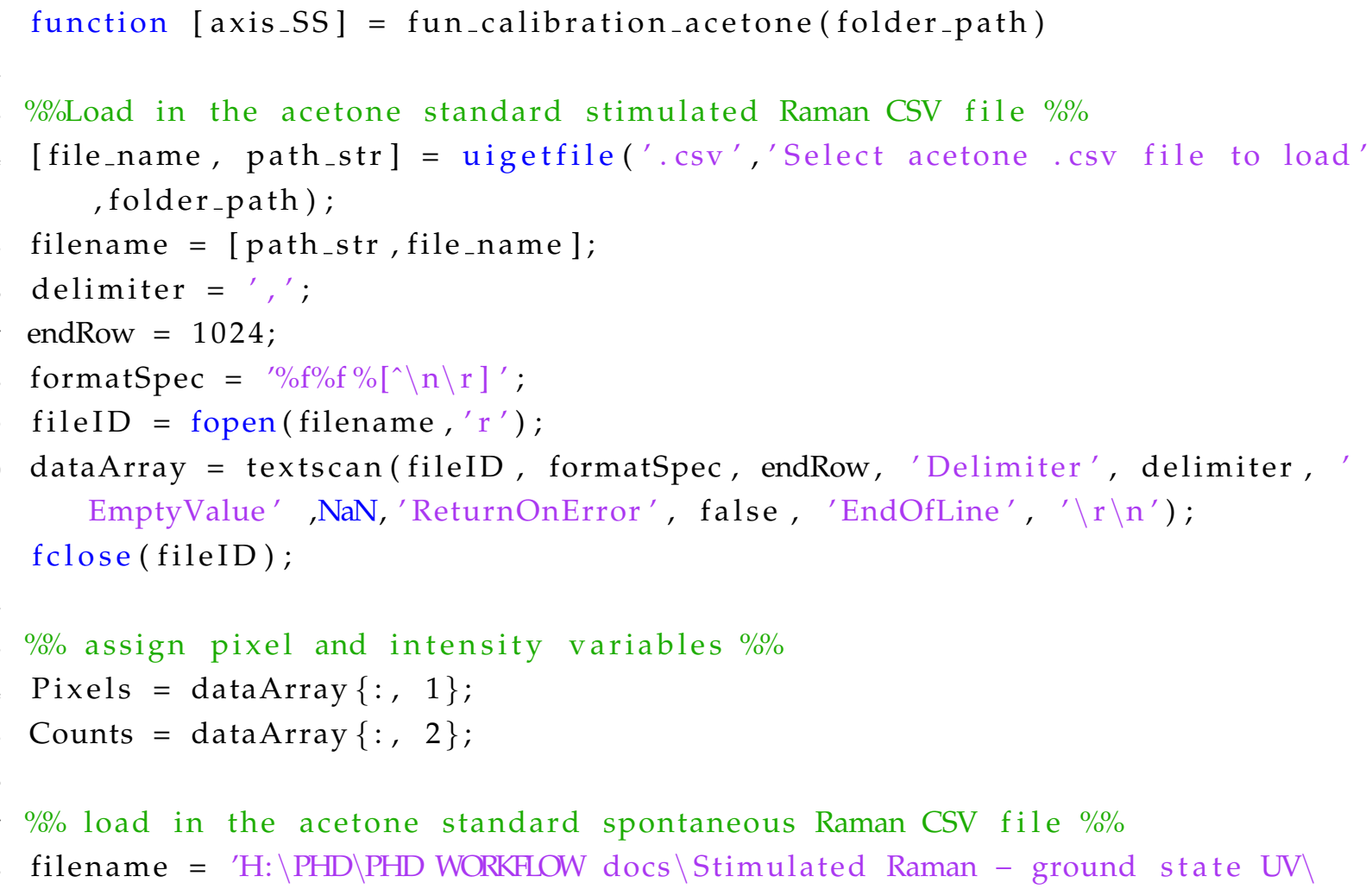


Real Raman $\backslash 002$-acetone -514nm-1800-H600-x100Im-Acc5s-N15. txt ' ; 
59

60

\section{B.4 Calibration of the PDA (Stresing) detector using three notch filters}

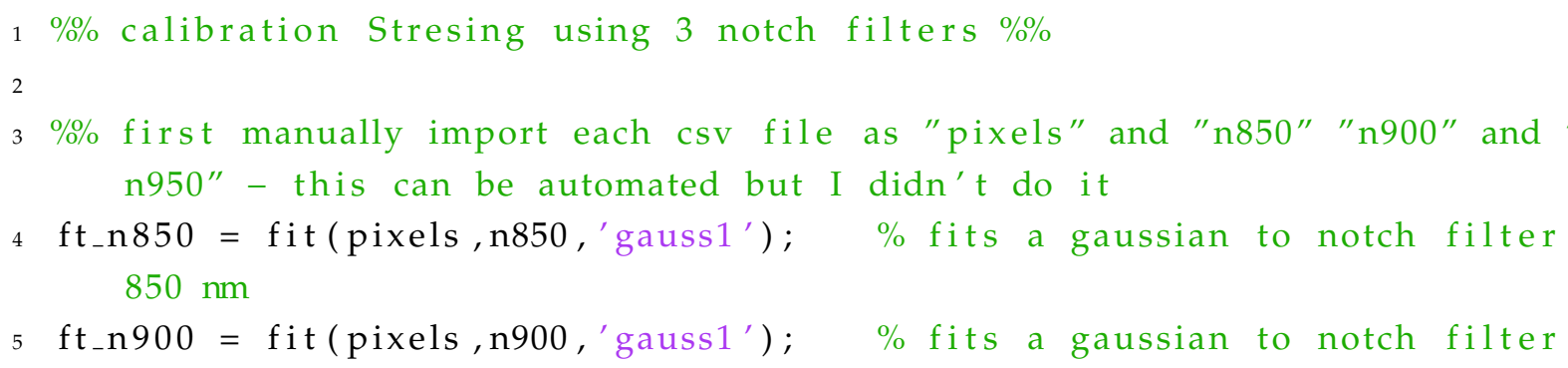


$900 \mathrm{~nm}$

$6 \mathrm{ft}_{-} \mathrm{n} 950=\mathrm{fit}($ pixels, n950,' gauss1'); \% fits a gaussian to notch filter

$950 \mathrm{~nm}$

$\%$ check the fit \%

figure (1)

clf

hold on

grid on

plot (pixels, n850)

plot (pixels, n900)

plot (pixels, n950)

legend ('850 nm', '900 nm', '950 nm')

plot (ft_n850)

$\operatorname{plot}\left(\mathrm{ft}_{-} \mathrm{n} 900\right)$

plot ( $\left.\mathrm{ft}_{-} \mathrm{n} 950\right)$

legend ('850 nm fit', '900 nm fit', '950 nm fit')

$\%$ find the maximum of the fitted Gaussian peaks \%

coeficients $=\left[\right.$ coeffvalues $\left(\mathrm{ft}_{\_} \mathrm{n} 850\right)$ coeffvalues ( ft_n900) coeffvalues ( $\left.\mathrm{ft}_{-} \mathrm{n} 950\right)$ ];

max_850 = coeficients $(1) ;$

$\max _{-} 900=$ coeficients $(4)$

max_950 = coeficients $(7)$;

$\%$ find the closet pixel to the peaks \%

$\left[\sim, \operatorname{pix} \_850\right]=\min \left(\operatorname{abs}\left(\max _{-} 850-\mathrm{n} 850\right)\right)$;

$\left[\sim, p_{1}{ }^{2}+900\right]=\min \left(\operatorname{abs}\left(\max _{-} 900-\mathrm{n} 900\right) ;\right.$

$\left[\sim, \operatorname{pix} \_950\right]=\min \left(\operatorname{abs}\left(\max _{-} 950-\mathrm{n} 950\right)\right)$;

$\%$ make the pixel locations and the wavelength numbers in two vectors \%\%

pixel = [pix_850, pix_900, pix_950];

lamnda $=[850,900,950]$

$\%$ fit a bi-polynomial through the peaks \%\%

$\mathrm{P}=$ polyfit (pixel, lamnda, 2$)$

$\%$ convert to nm using polyfit coefficients \%

pixels = $1: 252$

axis $\_n m=P(1) *$ pixels $\wedge^{\wedge} 2+P(2) *$ pixels $+P(3)$;

3

$\%$ check the calibration $\% \%$

figure (2)

clf

Page 162 


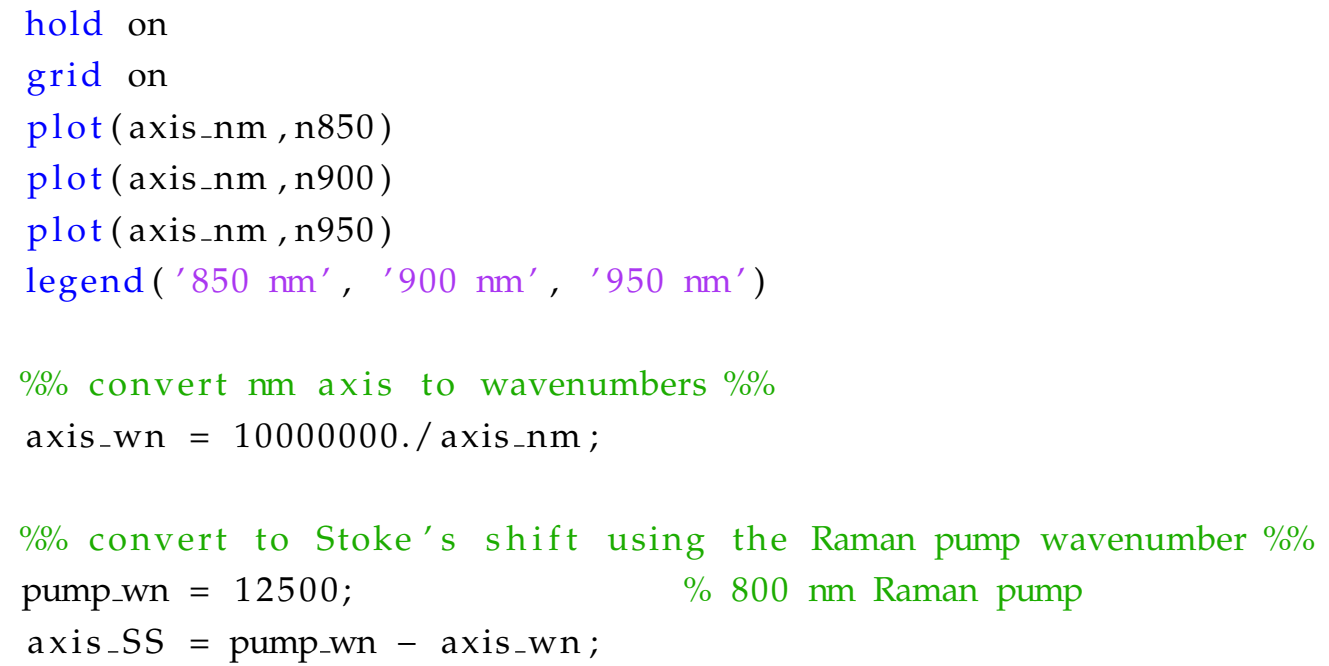

\section{B.5 Baseline removal using asymmetric least squares ap- proximation}

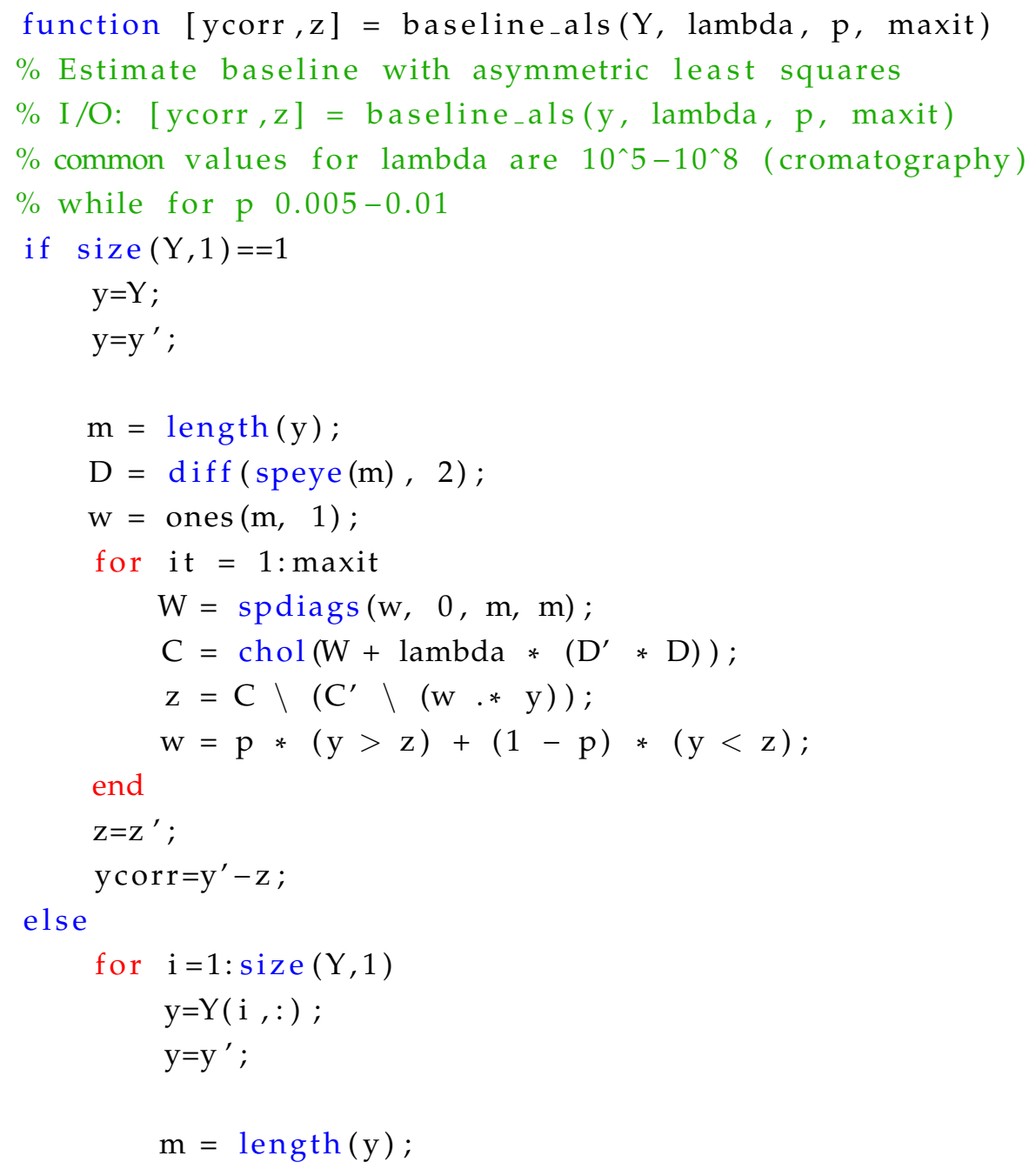




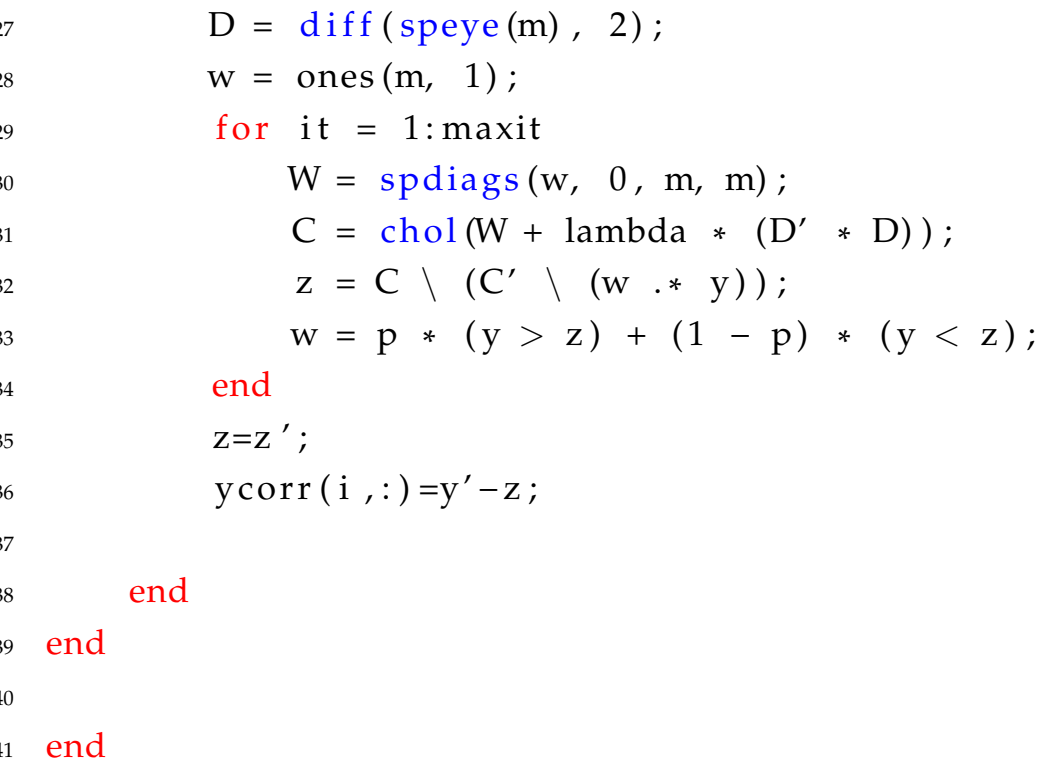




\section{Appendix C}

\section{Matlab code used for FSRS data post-processing}

\section{C.1 Derivative analysis of the FSRS surfaces}

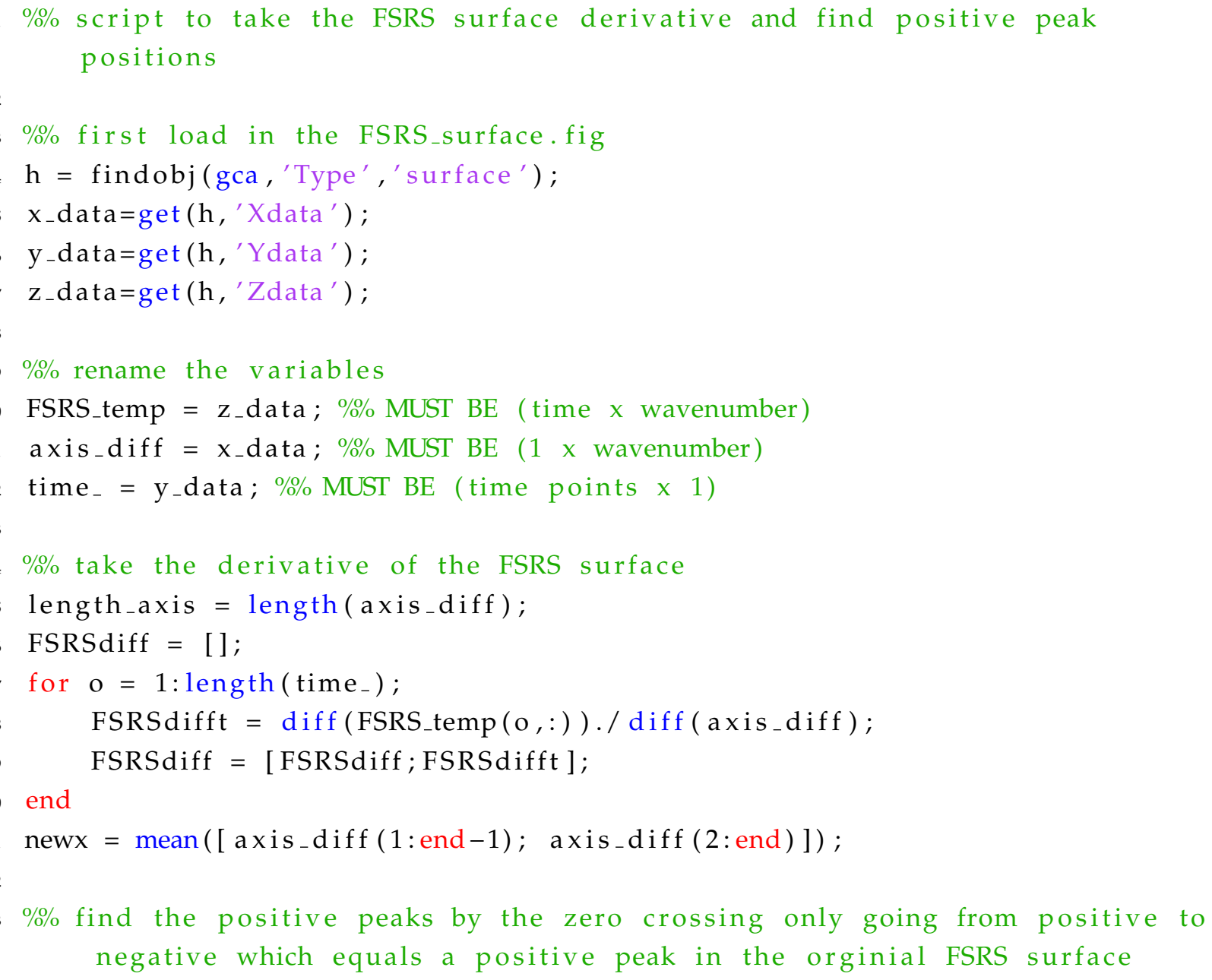




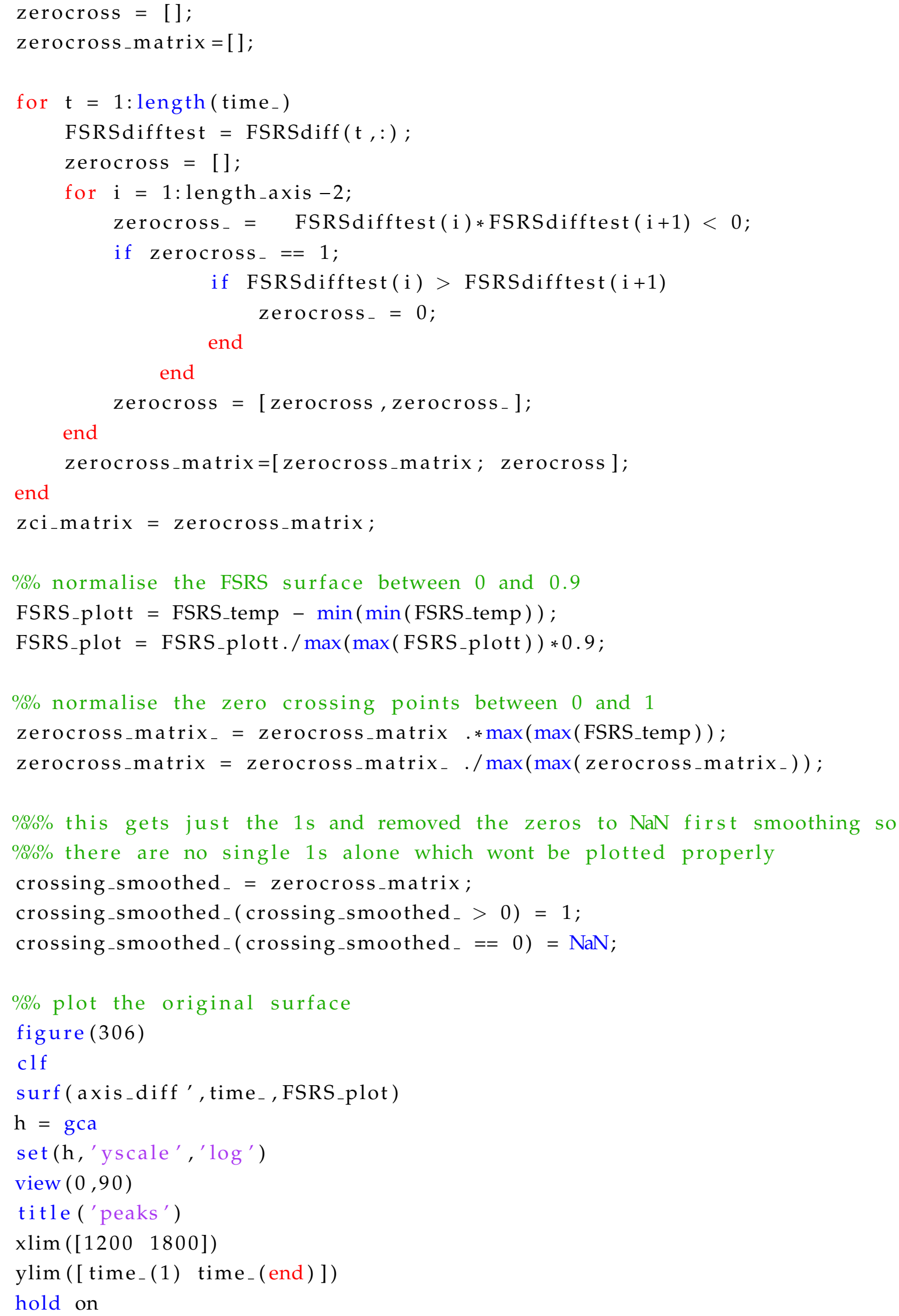

Page 166 


\section{C.1. Derivative analysis of the FSRS surfaces}

68

$69 \%$ plot the zero crossing points

$70 \operatorname{surf}\left(\right.$ newx $(1 \text { : length_axis }-2)^{\prime}$, time t $_{-}$, crossing_smoothed $\left._{-}\right)$ 


\section{Appendix D}

\section{Vibrational mode assignments of DHICA}


DHICA carboxylated form

Peak 1

Peak 2

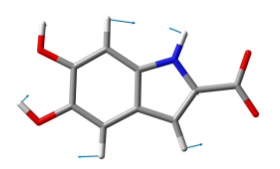

$1249 \mathrm{~cm}^{-1}$

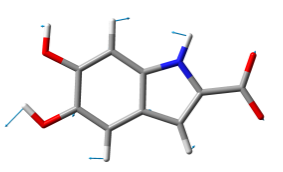

$1350 \mathrm{~cm}^{-1}$

Peak 3

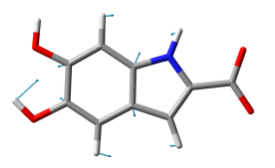

$1415 \mathrm{~cm}^{-1}$

Peak 4

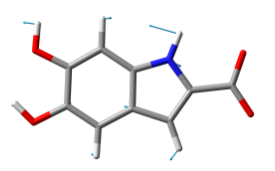

$1463 \mathrm{~cm}^{-1}$

Peak 5

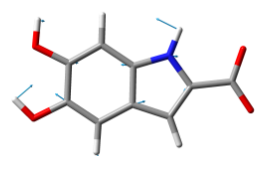

$1561 \mathrm{~cm}^{-1}$

Peak 6

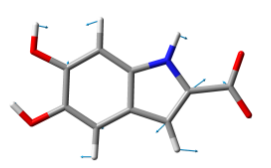

$1610 \mathrm{~cm}^{-1}$

Peak 7

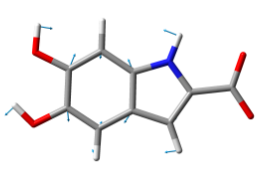

$1673 \mathrm{~cm}^{-1}$

Peak 8

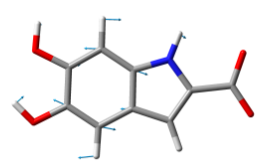

$1725 \mathrm{~cm}^{-1}$
DHICA phenolate di-anion
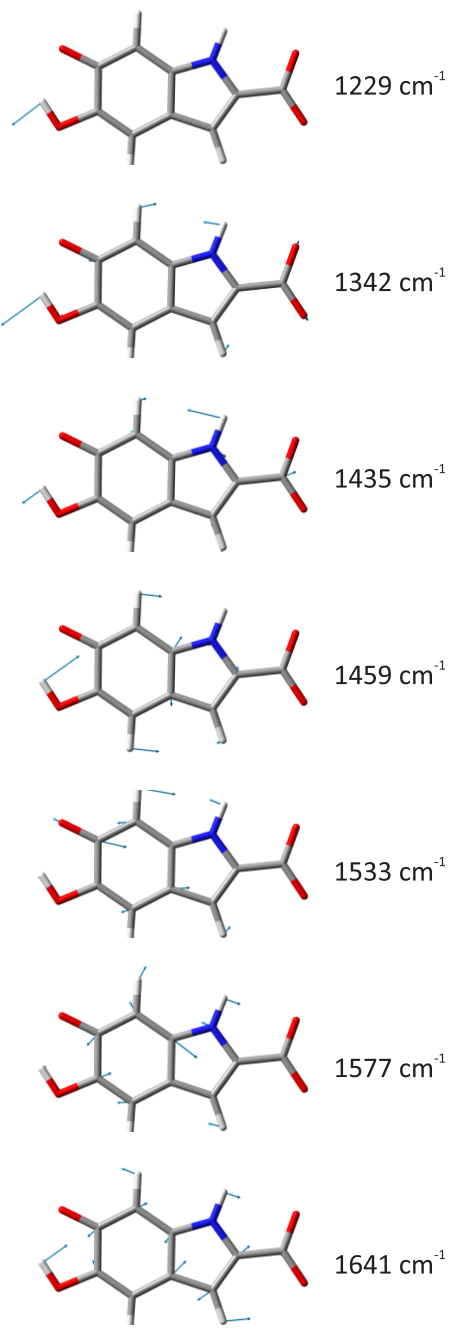

Figure D.1: The vibrational mode assignments for the carboxylate anion and phenolate di-anion calculated using DFT are graphically presented. Each peak is labelled as peak 1 - peak 8 as a reference to the frequencies identified in figure 5.12 . We see here that the vibrations are similar in nature, due to there closely lying frequencies. 


\section{Appendix E}

\section{Pseudo-Voigt model for steady state mode assignments}

\section{E.1 Pseudo-Voigt model parameters used for the ground state melanin film}

The Matlab code written for the pseudo-Voigt analysis of the eumelanin ground state Raman spectrum is presented in section E.2 of this appendix. There are 3 key parameters used in generating pseudo-Voigt distributions, which are a convolution of Lorentzian and Gaussian functions ${ }^{[154]}$ : the peak amplitude, FWHM, and central peak frequency. The central peak frequency is the same for both the Lorenztian and Gaussian functions, but the FWHM values and amplitudes of each are set individually.

\begin{tabular}{|l|l|l|l|l|}
\hline \multicolumn{5}{|c|}{ pseudo-Voigt parameters used to model the eumelanin ground stat Raman spectrum } \\
\hline \hline frequency $\left(\mathrm{cm}^{-1}\right)$ & $\mathrm{amp}_{\text {Gauss. }}$ & FWHM $_{\text {Gauss. }}\left(\mathrm{cm}^{-1}\right)$ & $\mathrm{amp}_{\text {Lorentz. }}$ & FWHM $_{\text {Lorentz. }}\left(\mathrm{cm}^{-1}\right)$ \\
\hline \hline 1220 & 0.000045 & 70 & 0.00001 & 200 \\
\hline 1341 & 0.00018 & 50 & 0.000098 & 135 \\
\hline 1418 & 0.0000085 & 150 & 0.000696 & 45 \\
\hline 1510 & 0.000360 & 100 & 0.0002 & 170 \\
\hline 1590 & 0.000140 & 1 & 0.000006 & 140 \\
\hline 1690 & 0.00004 & 160 & 0.00001 & 100 \\
\hline \hline
\end{tabular}

Table E.1: Pseudo-Voigt function parameters used to model the eumelanin pigment ground state Raman spectrum. 
The central peak frequencies are chosen in accordance with literature, where DFT analysis is used to assign the probable peak frequencies within the eumelanin pigment's ground state Raman spectral envelope ${ }^{[1551156}$. The amplitude and FWHM parameters are adjusted in a step-wise fashion so that the sum of the 6 pseudo-Voigt functions fit the ground state Raman spectral envelope shown in figure 6.6. The finalised variables used to generate the pseudo-Voigt functions are summarised in table E.1

\section{E.2 pseudo-Voigt model code used for the ground state melanin film}

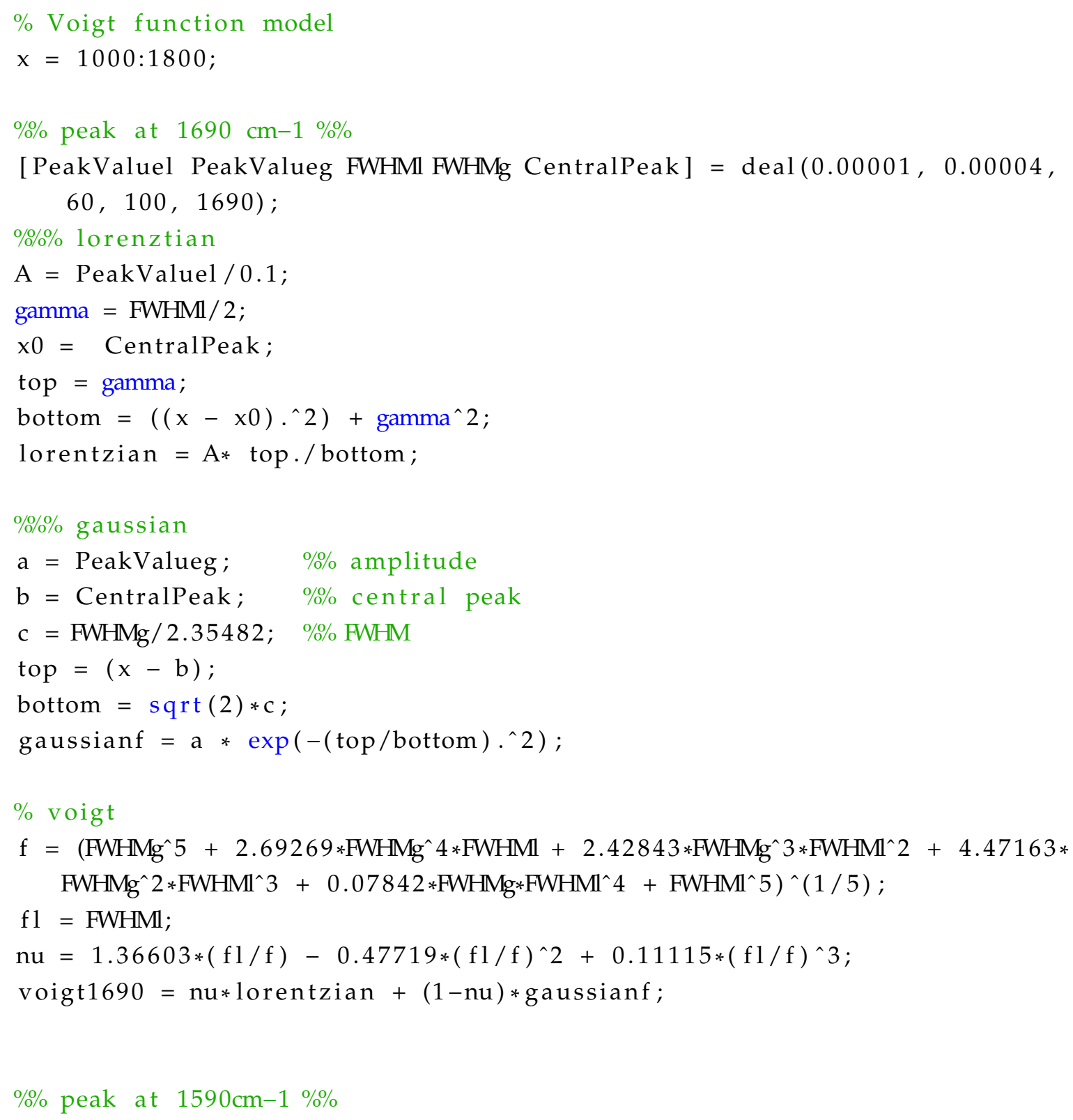

Page 172 
[PeakValuel PeakValueg FWHM FWHMg CentralPeak] = deal $(0.000006,0.000140$, $1,140,1590)$;

\section{$\% \%$ lorenztian}

$\mathrm{A}=$ PeakValuel /0.1;

gamma $=$ FWHMl $/ 2$;

$\mathrm{x} 0=$ CentralPeak;

top = gamma;

bottom $=\left((x-x 0) \cdot{ }^{\wedge} 2\right)+$ gamma^2;

lorentzian $=A *$ top./bottom;

$\%$ gaussian \%

$\mathrm{a}=$ PeakValueg; $\quad \%$ amplitude

$\mathrm{b}=$ CentralPeak; $\%$ central peak

c $=\mathrm{FWHMg} / 2.35482 ; \% \mathrm{FWHM}$

top $=(x-b)$;

bottom $=\operatorname{sqrt}(2) * \mathrm{c}$;

gaussianf $=a * \exp \left(-(\right.$ top $/$ bottom $\left.) \cdot{ }^{\wedge} 2\right)$;

$\%$ voigt \% $\%$

$\mathrm{f}=\left(\mathrm{FWHMg}^{\wedge} 5+2.69269 * \mathrm{FWHMg}^{\wedge} 4 * \mathrm{FWHMl}+2.42843 * \mathrm{FWHMg}^{\wedge} 3 * \mathrm{FWHM}^{\wedge} 2+4.47163 *\right.$ $\left.\mathrm{FWHMg}^{\wedge} 2 * \mathrm{FWHMl}^{\wedge} 3+0.07842 * \mathrm{FWHMg}^{2} \mathrm{FWHM}^{\wedge} 4+\mathrm{FWHM}^{\wedge} 5\right)^{\wedge}(1 / 5) ;$

$\mathrm{fl}=\mathrm{FWHMl}$;

$\mathrm{nu}=1.36603 *(\mathrm{fl} / \mathrm{f})-0.47719 *(\mathrm{fl} / \mathrm{f}) \wedge 2+0.11115 *(\mathrm{fl} / \mathrm{f}) \wedge 3$;

voigt1590 = nu*lorentzian $+(1-n u) *$ gaussianf;

$\%$ peak at $1510 \mathrm{~cm}-1 \%$

[PeakValuel PeakValueg FWHM FWHMg CentralPeak] = deal $(0.0002,0.000360$, $100,170,1510)$;

$\% \%$ lorenztian

$\mathrm{A}=$ PeakValuel / 0.1;

gamma $=\mathrm{FWHM} / 2$;

$\mathrm{x} 0=$ CentralPeak;

top = gamma;

bottom $=\left((x-x 0) \cdot{ }^{\wedge} 2\right)+$ gamma^$^{\wedge}$;

lorentzian $=A *$ top. $/$ bottom;

$\% \%$ gaussian

a = PeakValueg; $\quad \%$ amplitude

$\mathrm{b}=$ CentralPeak; $\%$ central peak

c $=\mathrm{FWHMg} / 2.35482 ; \%$ FWHM

top $=(x-b)$;

bottom $=\operatorname{sqrt}(2) * c$;

gaussianf $=a * \exp (-($ top $/$ bottom $) . \wedge 2) ;$ 


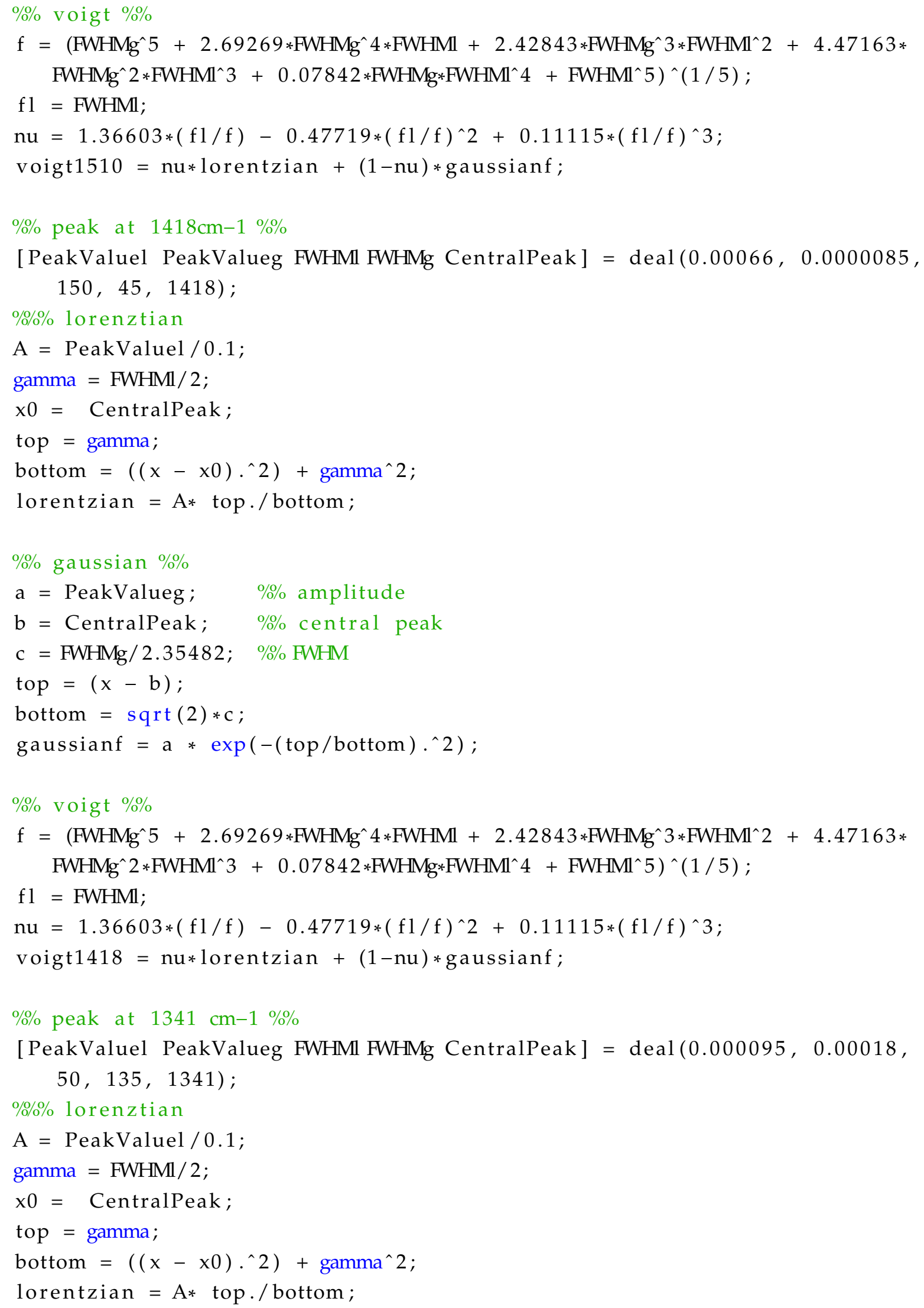




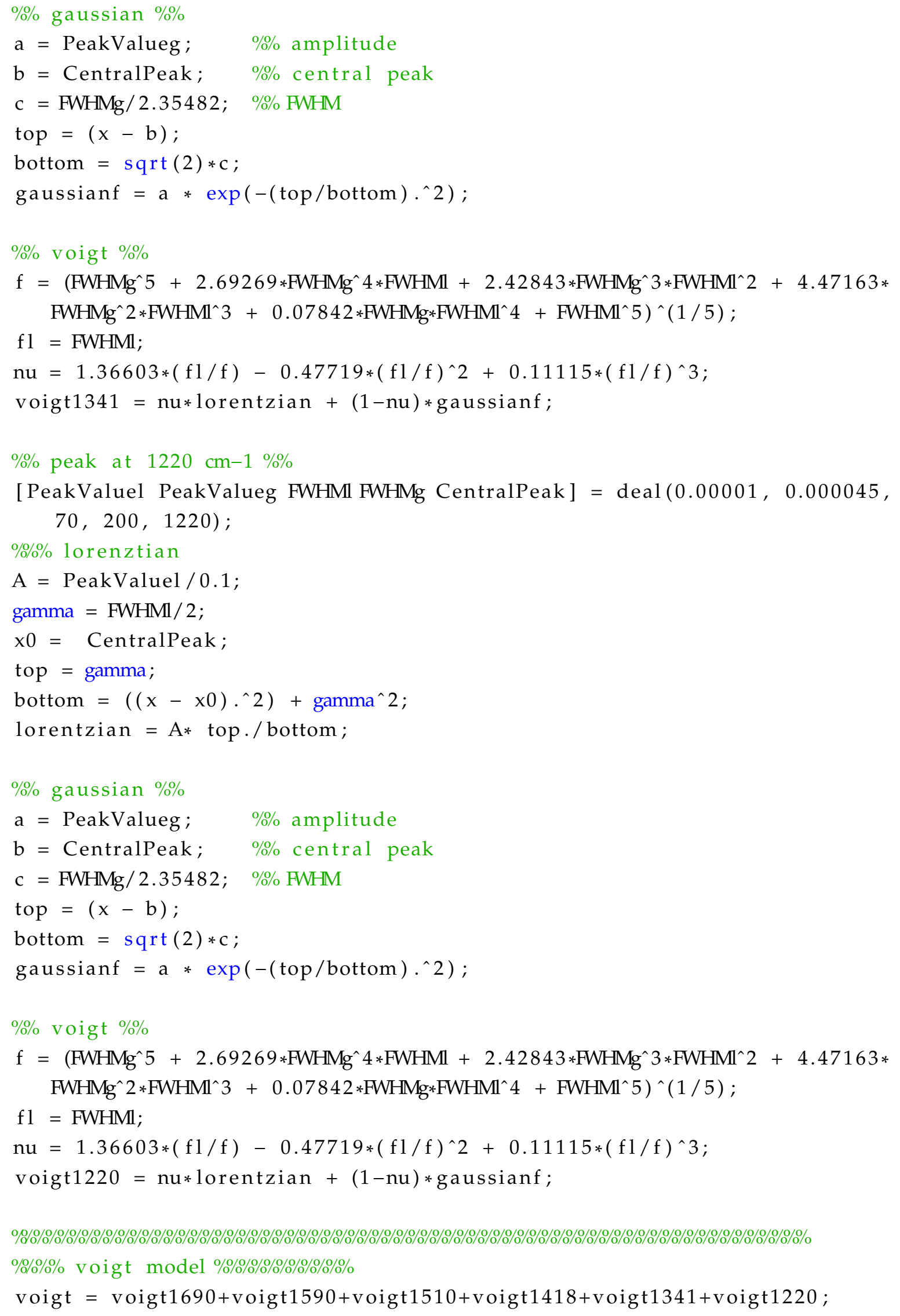




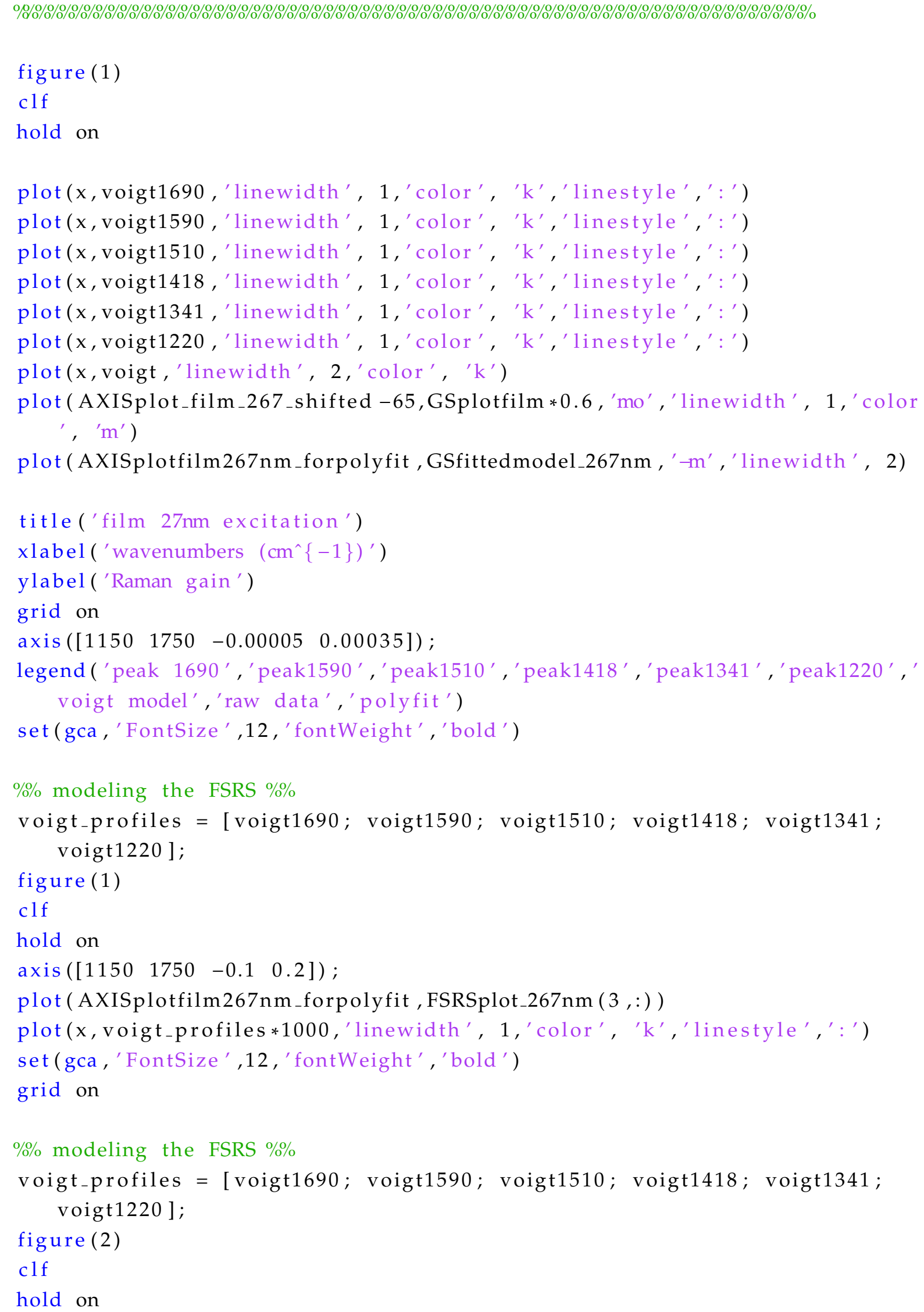

Page 176 
192 axis $\left(\left[\begin{array}{llll}1150 & 1750 & -0.1 & 2\end{array}\right]\right)$;

$193 \%$ plot (AXISplotfilm267nm_forpolyfit, FSRSplot_267nm (3, : ) )

194 plot (AXISplotfilm267nm_forpolyfit, FSRSplot_267nm_norm (2:end, : ) )

195 plot (x, voigt_profiles*10000,'linewidth', 1 ,' color', 'k',' linestyle', ': ')

196 set (gca, 'FontSize',12, 'fontWeight', 'bold')

197 grid on 


\section{Appendix F}

\section{SVD analysis of eumelanin films and dispersions}

In both the film and eumelanin sample preparations, for excitation wavelengths at 267 $\mathrm{nm}$ and $400 \mathrm{~nm}$, two spectral components are isolated using SVD analysis, introduced in section 4.5.5.1 of chapter 4 , and their kinetics extracted accordingly. These operations are performed in Maltab using the multivariate curve resolution MCR-ALS 2.0 toolbox algorithm ${ }^{[132]}$. In the following sections, FSRS surfaces for each experimental configuration are presented, along with their spectral and kinetic SVD analyses.

\section{F.1 Comparison of the ground and excited state}

Comparing the ground and excited state Raman spectra of eumelanin reveals key differences. The ground state Raman spectrum features two main frequency bands which are broad $\sim 100 \mathrm{~cm}^{-1}$ FWHM in nature. This spectrum is compared to amorphous carbon giving evidence of the heterogeneous nature of the eumelanin molecule. Similarly, by observing the resonance Raman spectra showing a frequency dependence on Raman pump wavelength, and Voigt modelling, it can be concluded that the ground state Raman spectrum contains a variety of species with differing conjugation lengths, overlapping in frequency.

FSRS surfaces for eumelanin films, and the SVD component analysis thereof, are presented in figure F.1. The two surfaces shown in panels $a$ and $b$ show the raw data matrices collected for two different excitation wavelengths at $267 \mathrm{~nm}$ and $400 \mathrm{~nm}$. Immediately apparent are peak dynamics presenting on $\sim p s$ timescales, with slightly different spectral locations and rise/decay kinetics. Through SVD analysis, two main 
SVD analysis of eumelanin dispersion
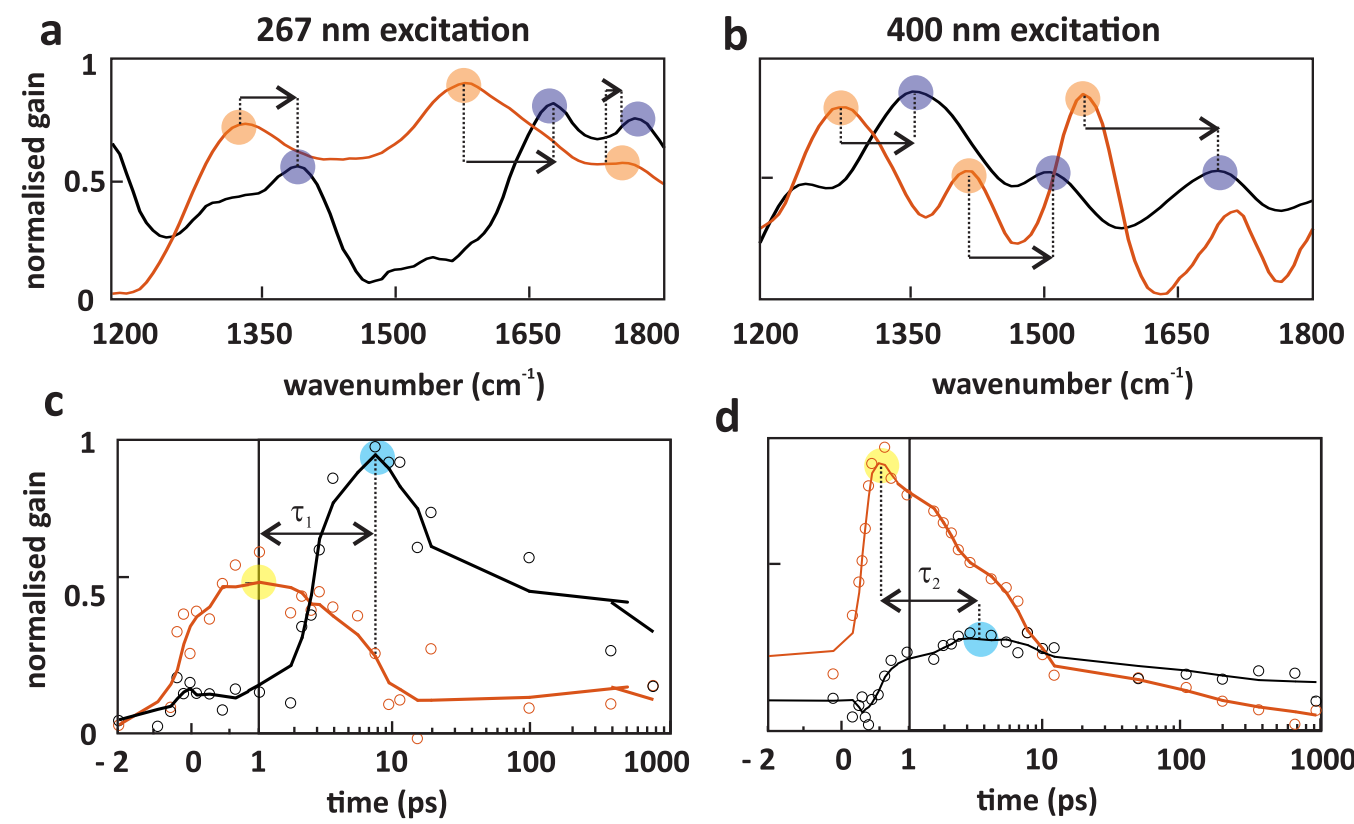

Figure F.1: Two SVD components extracted for melanin films at two excitation wavelengths, $267 \mathrm{~nm}$ and $400 \mathrm{~nm}$, are shown as the orange and black curves in panels $a$ and $b$. The peak positions are highlighted with circles as well as a shifting of these peaks between the two components, indicated by arrows. The kinetics for each spectral component are shown in panels $c$ and $d$, with the time rate of the shift shown as $\tau_{1}$ and $\tau_{2}$. The TA kinetics (a PIA band averaged across the spectrum) is given as the pink dashed curves, showing the excited state lifetime of the material

spectral components are extracted, shown in panels $c$ and $d$ for each excitation wavelength, with their dynamic signatures shown in panels $e$ and $f$. Here we see a conversion from the first spectral species rising, and then decaying with the rise of the second species. In both cases, blue-shifts are apparent around $1-2 p s$, indicating a transfer of energy from one species to the other, before relaxation into the ground state at around 1 ns for each experimental configuration.

It is first noted that the overall timescales of the FSRS dynamics are comparable to those of TA presented in section 6.2. Of interest is the nature of eumelanin's broad, overlapping Raman spectra in the ground state being in direct comparison with that of it's excited state vibrational signatures, which show localised excitations of specific frequencies. This indicates that the excited state manifold is specific to eumelanin's monomer (and oligomer) subunit structures. 
SVD analysis of eumelanin films
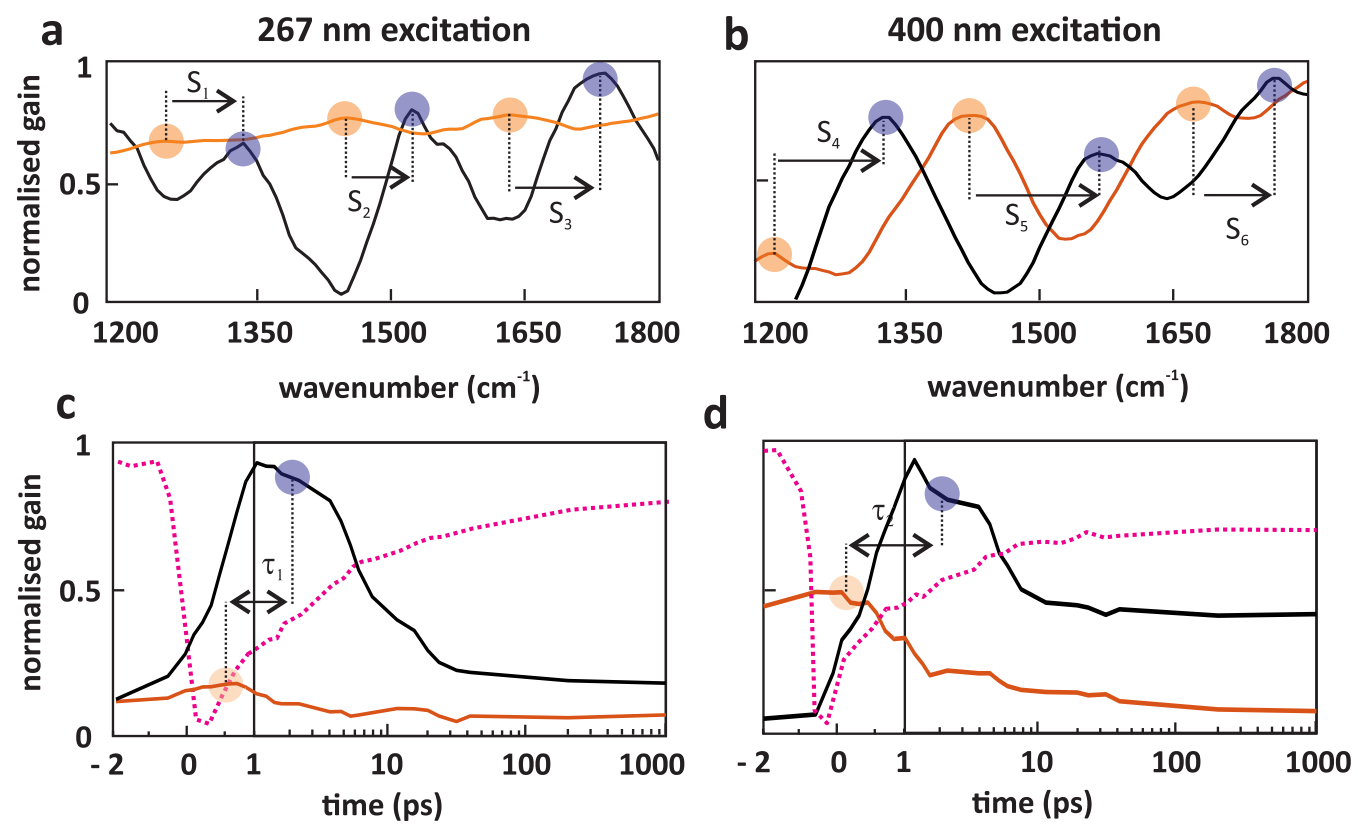

Figure F.2: Two SVD components extracted for melanin dispersions at two excitation wavelengths, $267 \mathrm{~nm}$ and $400 \mathrm{~nm}$, are shown as the orange and black curves in panels $a$ and $b$. The peak positions are highlighted with circles as well as a shifting of these peaks between the two components, indicated by arrows. The kinetics for each spectral component are shown in panels $c$ and $d$, with the time rate of the shift shown as $\tau_{1}$ and $\tau_{2}$. The TA kinetics (a PIA band averaged across the spectrum) is given as the pink dashed curves, showing the excited state lifetime of the material.

Here we see a distinct blue-shift, or "stiffening" of the vibrational modes over time. Table F.1 gives the extracted peak position shifts, and the changes to the peaks FWHM bandwidths extracted from the SVD component analysis using Gaussian deconvolution as shown in figures F.3 and F.4. Here, the two key spectral components (for each excitation wavelength) are resolved with 3 Gaussian functions, shown as $a, b$ and $c$ whose spectral shifts $\Delta a-c$ and peak FWHM bandwidths are also presented. The vibrational kinetics observed show good agreement with the excited state lifetime of the material provided by the TA kinetics in figure 6.1. The $\sim p s$ spectral shifts observed in the vibrational kinetics give evidence of a rapid structural change upon light excitation. 
SVD analysis - eumelanin film, $267 \mathrm{~nm}$ excitation
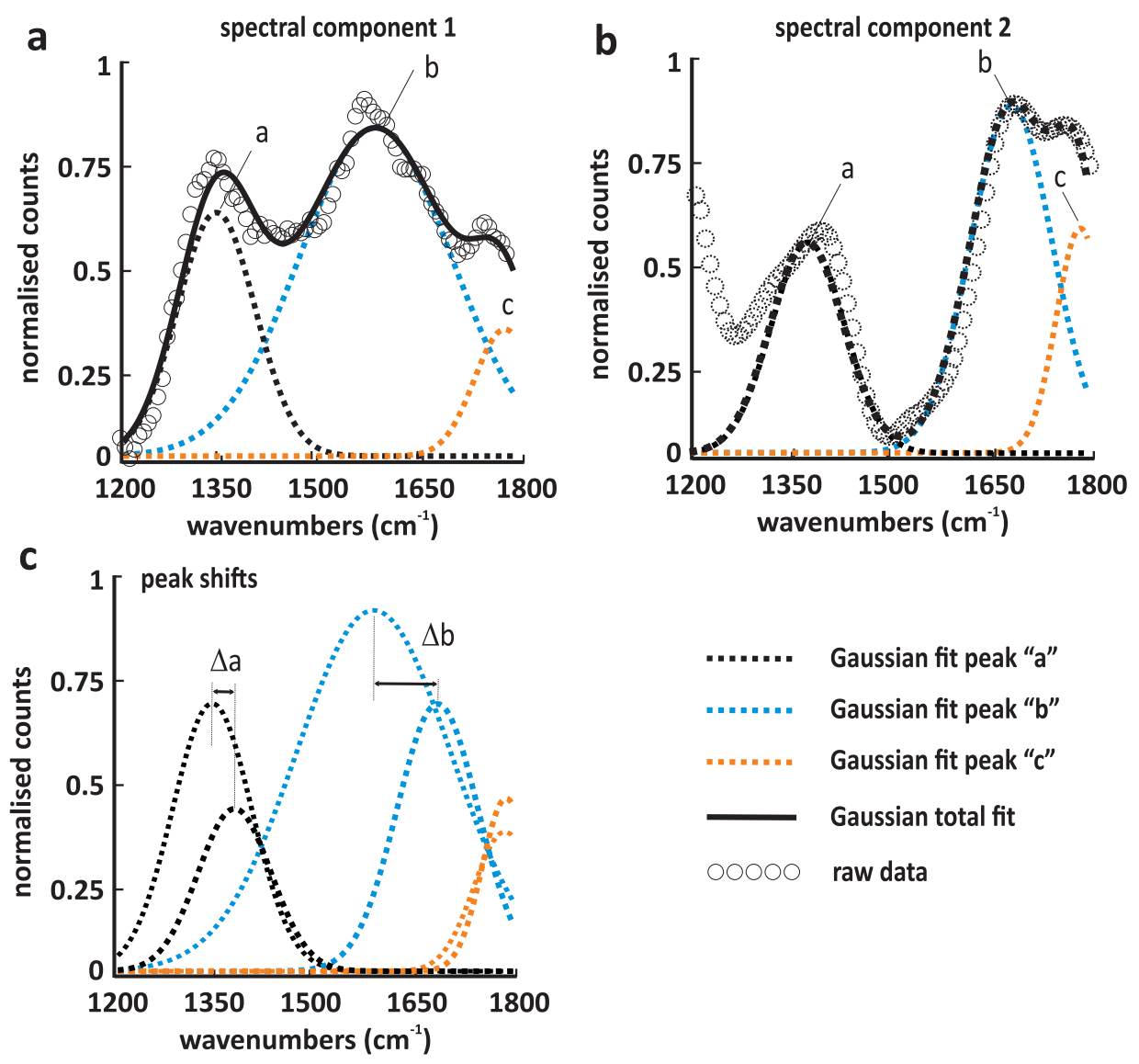

Figure F.3: FSRS SVD film analysis for a $267 \mathrm{~nm}$ excitation shows two spectral components shown in the panels a and b. A Gaussian deconvolution shows three Gaussian functions can be modelled to fit each spectral component, $a, b$ and $c$ as the black, blue and orange dashed curves. The Gaussian deconvolution for the first spectral feature is shown in panel $a$, with the peak positions being at $1343 \mathrm{~cm}^{-1}$, $1590 \mathrm{~cm}^{-1}$ and $1800 \mathrm{~cm}^{-1}$. The deconvolution for the second spectral component, shown in panel $b$, shows peak positions at $1379 \mathrm{~cm}^{-1}, 1687 \mathrm{~cm}^{-1}$ and $1800 \mathrm{~cm}^{-1}$ demonstrating a blue shift for the main two components $a$ and $b$ of $\sim 36 \mathrm{~cm}^{-1}$ and $\sim$ $97 \mathrm{~cm}^{-1}$ respectively. The higher energy peak does not appear to shift significantly between each component.

\section{F.1.0.1 FSRS of eumelanin in dispersion}

FSRS surfaces for eumelanin dispersions, and the SVD component analysis thereof, are presented in figure F.2 for excitation wavelengths of $267 \mathrm{~nm}$ and $400 \mathrm{~nm}$. The two 
SVD analysis - eumelanin film, $400 \mathrm{~nm}$ excitation
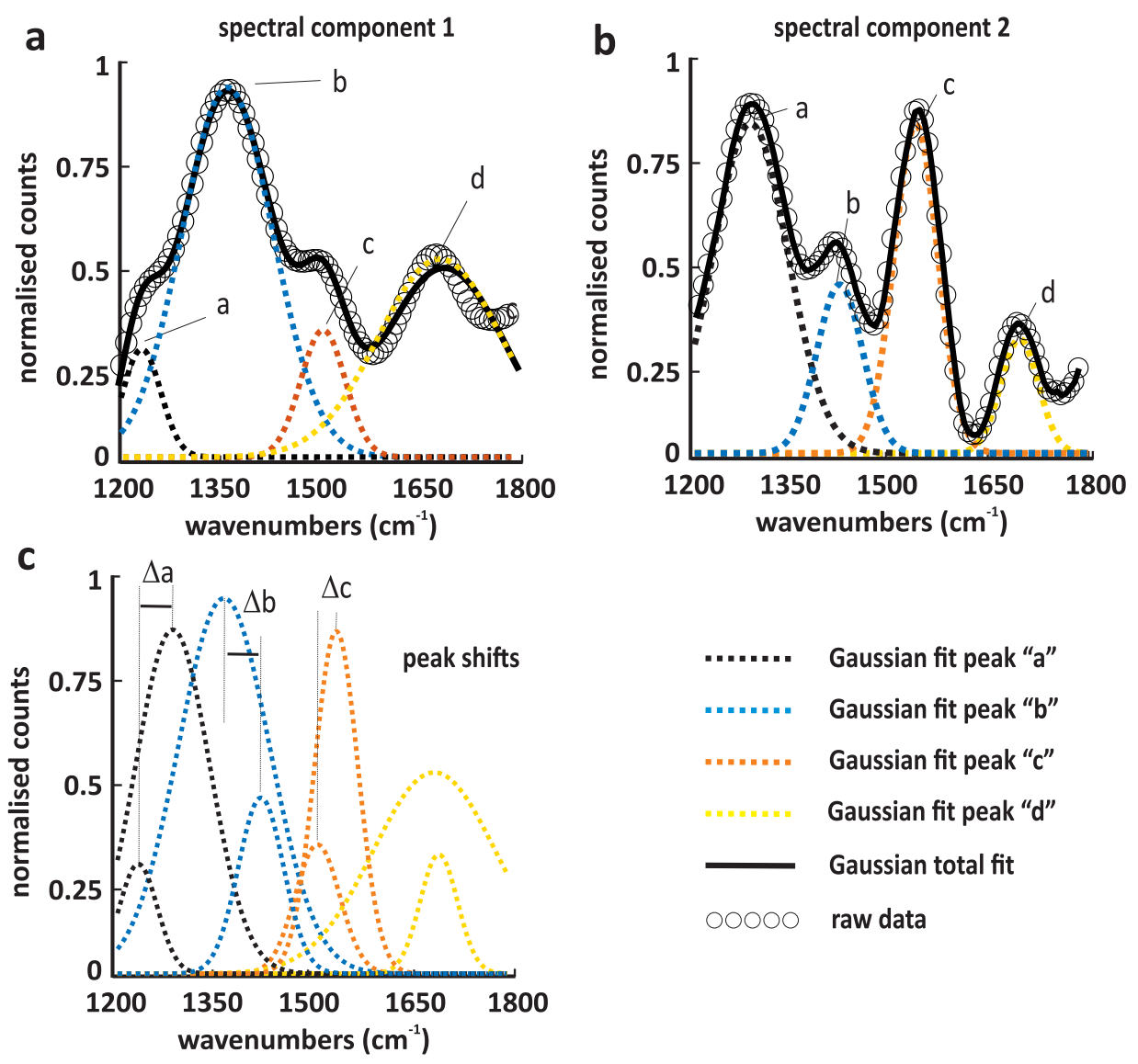

Figure F.4: SVD film analysis for eumelanin's FSRS data under $400 \mathrm{~nm}$ excitation reveals two spectral components shown in the panels $a$ and $b$. A Gaussian deconvolution of these components reveals four primary Gaussian functions, $a-d$. The peak positions relative to the two components are presented in panel $c$, with clear blue-shifts represented as $\Delta a-\Delta c$, with no apparent shift to the fourth $d$ component.

surfaces presented in panels $a$ and $b$ show the raw data matrices collected for two different excitation wavelengths at $267 \mathrm{~nm}$ and $400 \mathrm{~nm}$. Immediately apparent are peak dynamics presenting on $\sim 1-10 \mathrm{ps}$ timescales, with differing spectral locations and rise/decay kinetics. The surfaces show 5 - 6 distinct peaks under $267 \mathrm{~nm}$ excitation, and 3 dominant peaks under $400 \mathrm{~nm}$ excitation.

Table F.2 gives the peak values, peak shifts and bandwidth changes for the deconvolu- 


\begin{tabular}{|l|l|l|l|l|}
\hline \multicolumn{5}{|c|}{ Peak assignments from SVD spectra for eumelanin film } \\
\hline excitation $\lambda$ & comp. 1 peaks $\left(\mathrm{cm}^{-1}\right)$ & comp. 2 peaks $\left(\mathrm{cm}^{-1}\right)$ & $\Delta \mathrm{cm}^{-1}$ & $\Delta$ FWHM $\left(\mathrm{cm}^{-1}\right)$ \\
\hline \multirow{3}{*}{$267(\mathrm{~nm})$} & 1342 & 1377 & +35 & -3 \\
\cline { 2 - 5 } & 1586 & 1686 & +100 & -110 \\
\cline { 2 - 5 } & 1789 & 1789 & 0 & unresolved \\
\hline \multirow{3}{*}{$400(\mathrm{~nm})$} & 1230 & 1282 & +52 & +43 \\
\cline { 2 - 5 } & 1364 & 1418 & +54 & -78 \\
\cline { 2 - 5 } & 1510 & 1537 & +27 & -6 \\
\cline { 2 - 5 } & 1690 & 1690 & 0 & -155 \\
\hline
\end{tabular}

Table F.1: This table shows the peak locations extracted from the Gaussian deconvolution of the 2 SVD components for eumelanin films at two excitation wavelengths $267 \mathrm{~nm}$ and $400 \mathrm{~nm}$. The second and third columns are the peak locations for components 1 and 2 (denoted as "comp. 1" and "comp. 2"). The fourth column is the change in wavenumber between each peak, while the last column shows the change in FWHM bandwidth for each.

tion analysis under both wavelength excitations.

\begin{tabular}{|l|l|l|l|l|}
\hline \multicolumn{5}{|c|}{ Peak assignments from SVD spectra for eumelanin dispersion } \\
\hline excitation $\lambda$ & comp. 1 $\left(\mathrm{cm}^{-1}\right)$ & comp. 2( $\left.\mathrm{cm}^{-1}\right)$ & $\Delta \mathrm{cm}^{-1}$ & $\Delta$ FWHM $\left(\mathrm{cm}^{-1}\right)$ \\
\hline \multirow{3}{*}{$267(\mathrm{~nm})$} & 1292 & 1350 & +58 & -44 \\
\cline { 2 - 5 } & 1456 & 1539 & +83 & -23 \\
\cline { 2 - 5 } & 1635 & 1729 & +94 & +11 \\
\hline \multirow{3}{*}{$400(\mathrm{~nm})$} & 1300 & 1400 & +100 & -16 \\
\cline { 2 - 5 } & 1357 & 1463 & +106 & -9 \\
\cline { 2 - 6 } & 1574 & 1655 & +81 & +4 \\
\hline
\end{tabular}

Table F.2: This table shows the peak locations extracted from the Gaussian deconvolution of the 2 SVD components for eumelanin dispersions at two excitation wavelengths $267 \mathrm{~nm}$ and $400 \mathrm{~nm}$. The second and third columns are the peak locations for components 1 and 2 (denoted as "comp. 1" and "comp. 2"). The fourth column is the change in wavenumber between each peak, while the last column shows the change in FWHM bandwidth for each. 

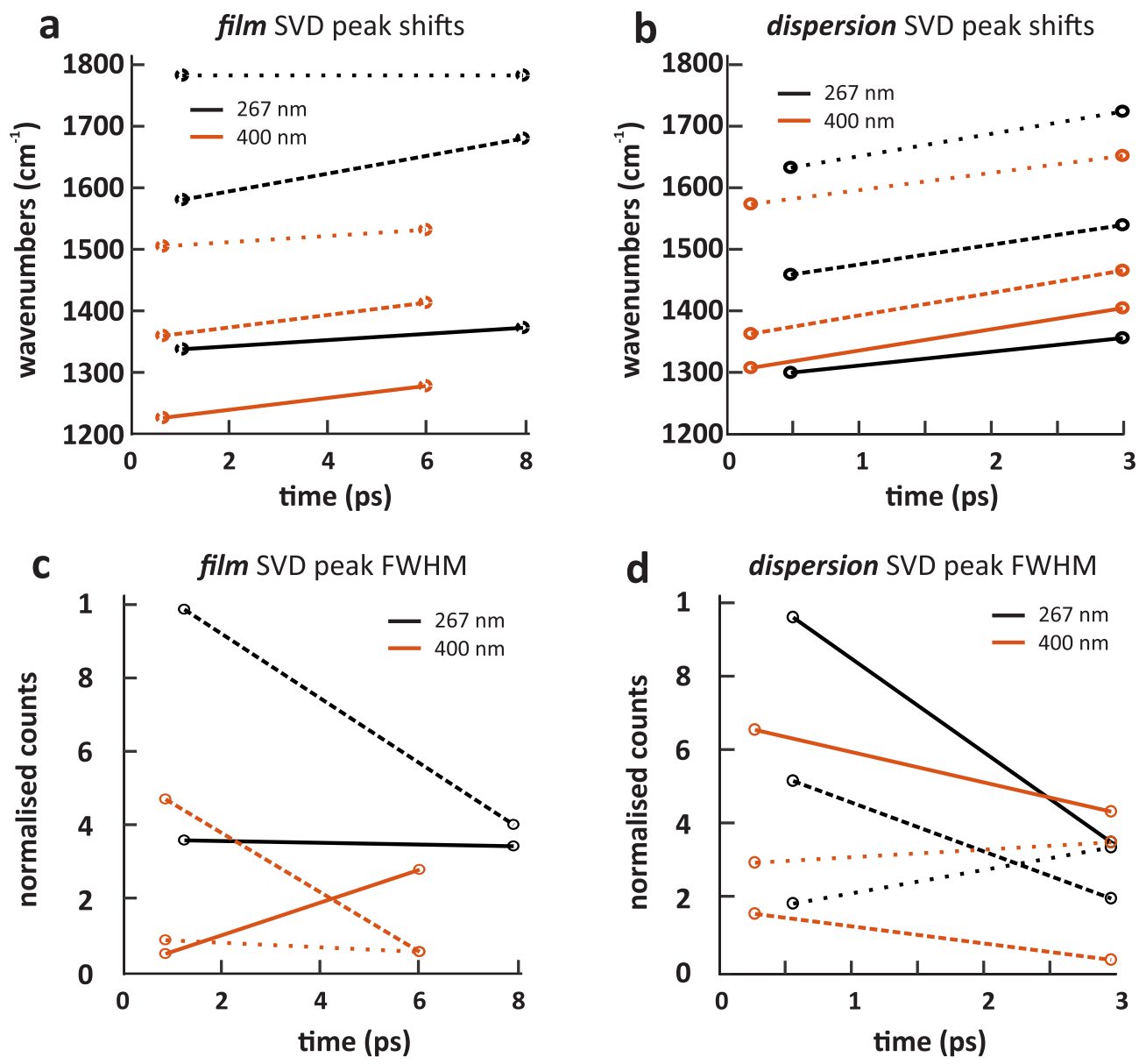

Figure F.5: This figure details the analysis of wavelength excitation for eumelanin's films and dispersions. Previously, the two states observed experimentally were compared within their own data-sets, but here we observe trends in the central peak shifts of the " $\mathrm{D}$ " and " $\mathrm{G}$ " -like bands, as a function of excitation wavelength. The left hand panels $a$ and $c$ show the peak shifts for both bands, with the orange lines being $400 \mathrm{~nm}$ excitation, and the black lines being $267 \mathrm{~nm}$ excitation. The lower panels illustrated changes to the FWHM of each peak in the SVD analysis, with the dashed or dotted lines corresponding to the main peaks above. Similarly, the left hand panels $b$ and $d$ show the same data for eumelanin in dispersion.

\section{F.1.0.2 Comparison of energy transfer for films and dispersions}

A list of peak positions, wavenumber shifts and bandwidths calculated from the SVD analysis, are detailed in tables F.1 (film) and F.2 (dispersion). A graphical comparison of these results as a function of excitation wavelength in shown in figure F.5. 
Here, specific trends can be observed. In panel $a$ of this figure, a comparison of the peak shifts for the film preparation is shown, with the black lines (dotted, dashed and solid) representing $267 \mathrm{~nm}$ excitation, while the orange lines represent $400 \mathrm{~nm}$ excitation. Here we can see a distinct upward frequency shifts of varying degrees for all identified peaks. The time scales for the x-axes are taken from the SVD kinetic peak positions. In panel $b$ of this figure, the same is presented for the dispersion data, and similar blue-shifting of all peak positions are observed - with more consistency (as shown by the more similar gradients of each line) than the peak shifts shown in panel $a$ for films.

It is of note the different time scales of the mode rise times and energy transfers for $\mathrm{UV}$ and visible excitations. For the film - with $400 \mathrm{~nm}$ excitation the initial modes peak at $\sim 0.7 \mathrm{ps}$, with the shifted modes peaking later at $\sim 6 \mathrm{ps}$, showing a transfer rate of energy of about $5.3 \mathrm{ps}$. The timescale is slightly different for the UV excitation, with the initial modes peaking at $\sim 1 \mathrm{ps}$, and the secondary modes peaking at $\sim 8 \mathrm{ps}$, showing a slower transfer of energy for the UV, and a longer lived excited state species. For the dispersion - the rise time of the initial modes are very similar to that of films, with the $400 \mathrm{~nm}$ excitation mode peaking at $\sim 0.2 \mathrm{ps}$ and the $267 \mathrm{~nm}$ mode peaking at $\sim 0.5 \mathrm{ps}$. The key difference is in the transfer of energy to the second mode, which occurs on a much faster time scale, with both excitations showing secondary mode peaks at $\sim 3 p s$. This means the transfer energy rate between the primary and secondary vibrational structures in dispersion happens much faster than in films, with a transfer rate of $\sim 2.5$ $p s$ in both cases.

Panels $c$ and $d$ of figure F.5 show the comparative changes of the peak bandwidths (at FWHM) for each mode in the SVD spectra. These bandwidths are fitted with Gaussian functions in the deconvolution analysis described earlier. Here we can see there is a less obvious correlation between the data, with some bandwidths decreasing in time and others increasing in time. 


\section{Appendix G}

\section{Eumelanin FSRS surfaces and differential analysis in ammonia dispersion}

This section presents FSRS data collected for eumelanin dispersions in ammonia, for two excitation wavelengths in the UV $(267 \mathrm{~nm})$ and the visible $(400 \mathrm{~nm})$ regions. The FSRS surfaces presented in figure G.1 are analysed using a derivative function as introduced in section 4.5.5.2 of chapter 4. The surfaces are also analysed using singular value decomposition (SVD) as described in section 4.5.5.1 of chapter 4 , with these results being presented in appendix $\mathrm{F}$.

The peak positions calculated from the FSRS surfaces using a differential analysis are plotted in figures G.1 and G.2 for 2 excitation pump wavelengths. The 2 excitation pumps in the UV and visible regions should selectively excite chromophores that differ from each other, and so the FSRS vibrational peaks are expected to differ between the two. Here we see that few comparison can be made between the surfaces. WE also observe that there are no obvious distinct frequency peak shifts occurring as observed in the film preparations, rather that the peaks appear to be consistently uniform across each surface. Observations of the surfaces alone show a more uniform rise and decay of frequency bands, occurring at slightly delayed times compared with that of the films, which is expected if the rapid ESPT decay pathway is not available. 
Appendix G. Eumelanin FSRS surfaces and differential analysis in ammonia dispersion

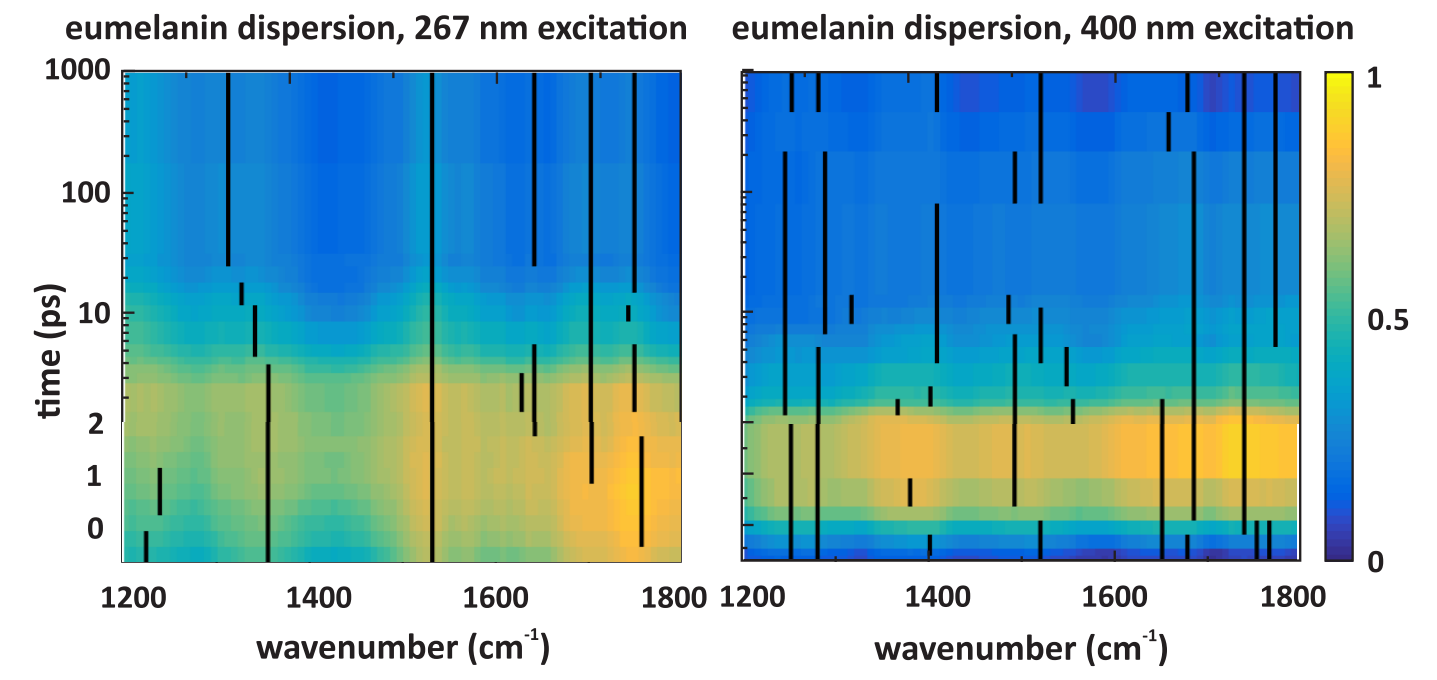

Figure G.1: FSRS surface of melanin dispersions for two excitation pump wavelengths. The top left panel is eumelanin dispersion at $267 \mathrm{~nm}$ and the top right panel the same but for $400 \mathrm{~nm}$ excitation.

\section{experimental peak shifts of eumelanin dispersion FSRS surfaces}
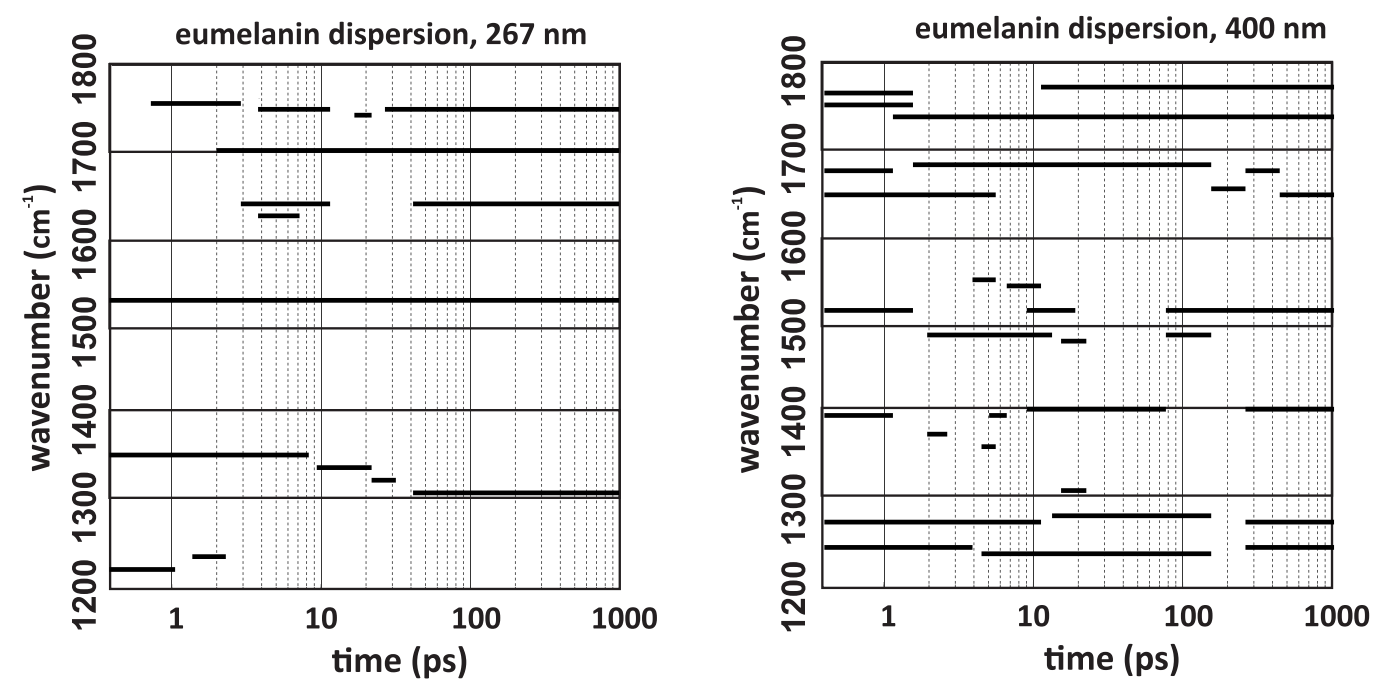

Figure G.2: Comparison of eumelanin dispersions at $267 \mathrm{~nm}$ and $400 \mathrm{~nm}$ excitations. The peak frequencies are plotted as a function of time. It is apparent that for both excitation pump wavelengths there are no clear frequency shifts occurring. It should be noted that all the peak positions captured using the differential analysis are presented, with the intensities of each peak not being explicitly shown. 


\section{Bibliography}

[1] N. Dissanayake and R. Mason, Effects of Ultraviolet Irradiation on Human SkinDerived Epidermal Cells In Vitro. Departments of Physiology and Animal Science, University of Sydney, NSW 2006, Australia: Journal of Cellular Physiology 157:119-127, 1993.

[2] F.-A. Miannay, Á. Bányász, T. Gustavsson, and D. Markovitsi, “Ultrafast excitedstate deactivation and energy transfer in guanine- cytosine dna double helices," Journal of the American Chemical Society, vol. 129, no. 47, pp. 14574-14575, 2007.

[3] N. K. Schwalb and F. Temps, "Base sequence and higher-order structure induce the complex excited-state dynamics in dna," Science, vol. 322, no. 5899, pp. 243$245,2008$.

[4] J. Léonard, T. Gelot, K. Torgasin, and S. Haacke, "Ultrafast fluorescence spectroscopy of biologically relevant chromophores using type ii difference frequency generation," in Journal of Physics: Conference Series, vol. 277, p. 012017, IOP Publishing, 2011.

[5] Statistics New Zealand, "Occurrence of melanoma." http:// archive.stats.govt.nz/browse_for_stats/environment/ environmental-reporting-series/environmental-indicators/ Home/Atmosphere-and-climate/skin-cancer.aspx, 2018. [Online; accessed 11-April-2018].

[6] N. Serpone, A. Salinaro, A. V. Emeline, S. Horikoshi, H. Hidaka, and J. Zhao, “An in vitro systematic spectroscopic examination of the photostabilities of a random set of commercial sunscreen lotions and their chemical uvb/uva active agents," Photochemical \& Photobiological Sciences, vol. 1, no. 12, pp. 970-981, 2002.

[7] C. Hayden, S. Cross, C. Anderson, N. Saunders, and M. Roberts, “Sunscreen penetration of human skin and related keratinocyte toxicity after topical application," Skin pharmacology and physiology, vol. 18, no. 4, pp. 170-174, 2005. 
[8] S. Butt and T. Christensen, "Toxicity and phototoxicity of chemical sun filters," Radiation protection dosimetry, vol. 91, no. 1-3, pp. 283-286, 2000.

[9] S. Brown, "Sunscreen wipes out corals," 2008.

[10] P. Meredith and T. Sarna, "The physical and chemical properties of eumelanin," Pigment Cell \& Melanoma Research, vol. 19, no. 6, pp. 572-594, 2006.

[11] M. d'Ischia, A. Napolitano, A. Pezzella, P. Meredith, and T. Sarna, "Chemical and structural diversity in eumelanins: unexplored bio-optoelectronic materials," Angewandte Chemie International Edition, vol. 48, no. 22, pp. 3914-3921, 2009.

[12] M. d'Ischia, K. Wakamatsu, F. Cicoira, E. Di Mauro, J. C. Garcia-Borron, S. Commo, I. Galván, G. Ghanem, K. Kenzo, P. Meredith, et al., "Melanins and melanogenesis: from pigment cells to human health and technological applications," Pigment cell E melanoma research, vol. 28, no. 5, pp. 520-544, 2015.

[13] A. Huijser, A. Pezzella, and V. Sundström, "Functionality of epidermal melanin pigments: current knowledge on uv-dissipative mechanisms and research perspectives," Physical chemistry chemical physics, vol. 13, no. 20, pp. 9119-9127, 2011.

[14] J. D'Orazio, S. Jarrett, A. Amaro-Ortiz, and T. Scott, "Uv radiation and the skin," International journal of molecular sciences, vol. 14, no. 6, pp. 12222-12248, 2013.

[15] G. Prampolini, I. Cacelli, and A. Ferretti, "Intermolecular interactions in eumelanins: a computational bottom-up approach. i. small building blocks," RSC Advances, vol. 5, no. 48, pp. 38513-38526, 2015.

[16] M. L. Tran, B. J. Powell, and P. Meredith, "Chemical and structural disorder in eumelanins: a possible explanation for broadband absorbance," Biophysical Journal, vol. 90, no. 3, pp. 743-752, 2006.

[17] P. Meredith, B. J. Powell, J. Riesz, S. P. Nighswander-Rempel, M. R. Pederson, and E. G. Moore, "Towards structure-property-function relationships for eumelanin," Soft Matter, vol. 2, no. 1, pp. 37-44, 2006.

[18] J. J. Riesz, The spectroscopic properties of melanin. University of Queensland Queensland, Australia, 2007.

[19] D. Tuna, A. Udvarhelyi, A. L. Sobolewski, W. Domcke, and T. Domratcheva, "Onset of the electronic absorption spectra of isolated and $\pi$-stacked oligomers 
of 5, 6-dihydroxyindole: an ab initio study of the building blocks of eumelanin," The Journal of Physical Chemistry B, vol. 120, no. 14, pp. 3493-3502, 2016.

[20] P. Meredith, C. Bettinger, M. Irimia-Vladu, A. Mostert, and P. E. Schwenn, “Electronic and optoelectronic materials and devices inspired by nature," Reports on Progress in Physics, vol. 76, no. 3, p. 034501, 2013.

[21] A. B. Mostert, B. J. Powell, F. L. Pratt, G. R. Hanson, T. Sarna, I. R. Gentle, and P. Meredith, "Role of semiconductivity and ion transport in the electrical conduction of melanin," Proceedings of the National Academy of Sciences, vol. 109, no. 23, pp. 8943-8947, 2012.

[22] J. P. Bothma, J. De Boor, U. Divakar, P. E. Schwenn, and P. Meredith, "Devicequality electrically conducting melanin thin films," Advanced Materials, vol. 20, no. 18, pp. 3539-3542, 2008.

[23] A. Bernardus Mostert, B. J. Powell, I. R. Gentle, and P. Meredith, "On the origin of electrical conductivity in the bio-electronic material melanin," Applied Physics Letters, vol. 100, no. 9, p. 093701, 2012.

[24] A. Pezzella, M. d'Ischia, A. Napolitano, A. Palumbo, and G. Prota, "An integrated approach to the structure of sepia melanin. evidence for a high proportion of degraded 5, 6-dihydroxyindole-2-carboxylic acid units in the pigment backbone," Tetrahedron, vol. 53, no. 24, pp. 8281-8286, 1997.

[25] J. B. Nofsinger, S. E. Forest, L. M. Eibest, K. A. Gold, and J. D. Simon, “Probing the building blocks of eumelanins using scanning electron microscopy," Pigment Cell Research, vol. 13, no. 3, pp. 179-184, 2000.

[26] P. Meredith and T. Sarna, "The physical and chemical properties of eumelanin," Pigment cell research, vol. 19, no. 6, pp. 572-594, 2006.

[27] A. v. Bondi, "van der waals volumes and radii," The Journal of physical chemistry, vol. 68, no. 3, pp. 441-451, 1964.

[28] C.-T. Chen, C. Chuang, J. Cao, V. Ball, D. Ruch, and M. J. Buehler, "Excitonic effects from geometric order and disorder explain broadband optical absorption in eumelanin," Nature communications, vol. 5, no. 1, pp. 1-10, 2014.

[29] R. Micillo, L. Panzella, K. Koike, G. Monfrecola, A. Napolitano, and M. d'Ischia, "“fifty shades" of black and red or how carboxyl groups fine tune eumelanin and 
pheomelanin properties," International Journal of Molecular Sciences, vol. 17, no. 5, p. 746, 2016.

[30] J. Riesz, J. Gilmore, and P. Meredith, "Quantitative photoluminescence of broad band absorbing melanins: a procedure to correct for inner filter and reabsorption effects," Spectrochimica Acta Part A: Molecular and Biomolecular Spectroscopy, vol. 61, no. 9, pp. 2153-2160, 2005.

[31] G. Vitiello, A. Pezzella, A. Zanfardino, M. Varcamonti, B. Silvestri, A. Costantini, F. Branda, and G. Luciani, "Titania as a driving agent for dhica polymerization: a novel strategy for the design of bioinspired antimicrobial nanomaterials," Journal of Materials Chemistry B, vol. 3, no. 14, pp. 2808-2815, 2015.

[32] J. Riesz, J. Gilmore, and P. Meredith, "Quantitative scattering of melanin solutions," Biophysical journal, vol. 90, no. 11, pp. 4137-4144, 2006.

[33] M. Kasha, "Characterization of electronic transitions in complex molecules," Discussions of the Faraday society, vol. 9, pp. 14-19, 1950.

[34] J. Riesz, J. Gilmore, and P. Meredith, "Quantitative photoluminescence of broad band absorbing melanins: a procedure to correct for inner filter and reabsorption effects," Spectrochimica Acta Part A: Molecular and Biomolecular Spectroscopy, vol. 61, no. 9, pp. 2153-2160, 2005.

[35] S. P. Nighswander-Rempel, J. Riesz, J. Gilmore, J. P. Bothma, and P. Meredith, "Quantitative fluorescence excitation spectra of synthetic eumelanin," The journal of physical chemistry B, vol. 109, no. 43, pp. 20629-20635, 2005.

[36] S. P. Nighswander-Rempel, I. B. Mahadevan, H. Rubinsztein-Dunlop, and P. Meredith, "Time-resolved and steady-state fluorescence spectroscopy of eumelanin and indolic polymers," Photochemistry and photobiology, vol. 83, no. 6, pp. 1449-1454, 2007.

[37] S. P. Nighswander-Rempel, J. Riesz, J. Gilmore, and P. Meredith, "A quantum yield map for synthetic eumelanin," The Journal of chemical physics, vol. 123, no. 19, p. 194901, 2005.

[38] P. Meredith and J. Riesz, "Radiative relaxation quantum yields for synthetic eumelanin," Photochemistry and photobiology, vol. 79, no. 2, pp. 211-216, 2004. 
[39] R. Berera, R. van Grondelle, and J. T. Kennis, “Ultrafast transient absorption spectroscopy: Principles and application to photosynthetic systems," Photosynthesis Research, vol. 101, no. 2-3, pp. 105-118, 2009.

[40] A. J. BARKER, K. CHEN, S. K. PRASAD, and J. M. HODGKISS, “High-sensitivity ultrafast transient absorption spectroscopy of organic photovoltaic devices," in Ultrafast Dynamics in Molecules, Nanostructures and Interfaces: Selected Lectures Presented at Symposium on Ultrafast Dynamics of the 7th International Conference on Materials for Advanced Technologies, pp. 36-52, World Scientific, 2013.

[41] F. R. K. A. T. H. Grieco, Christopher and B. Kohler, "Probing the heterogeneous structure of eumelanin using ultrafast vibrational fingerprinting.," Nature Communications, vol. 11, no. 1, 2020.

[42] J. B. Nofsinger, T. Ye, and J. D. Simon, "Ultrafast nonradiative relaxation dynamics of eumelanin," The Journal of Physical Chemistry B, vol. 105, no. 14, pp. 28642866, 2001.

[43] T. Ye and J. D. Simon, "Comparison of the ultrafast absorption dynamics of eumelanin and pheomelanin," The Journal of Physical Chemistry B, vol. 107, no. 40, pp. 11240-11244, 2003.

[44] M. J. Simpson, J. W. Wilson, F. E. Robles, C. P. Dall, K. Glass, J. D. Simon, and W. S. Warren, "Near-infrared excited state dynamics of melanins: the effects of iron content, photo-damage, chemical oxidation, and aggregate size," The journal of physical chemistry A, vol. 118, no. 6, pp. 993-1003, 2014.

[45] A. Corani, A. Pezzella, T. Pascher, T. Gustavsson, D. Markovitsi, A. Huijser, M. d'Ischia, and V. Sundstrom, "Excited-state proton-transfer processes of dhica resolved: From sub-picoseconds to nanoseconds," The journal of physical chemistry letters, vol. 4, no. 9, pp. 1383-1388, 2013.

[46] A. Huijser, A. Pezzella, J. K. Hannestad, L. Panzella, A. Napolitano, M. d'Ischia, and V. Sundström, "UV-dissipation mechanisms in the eumelanin building block DHICA," ChemPhysChem, vol. 11, no. 11, pp. 2424-2431, 2010.

[47] S. Olsen, J. Riesz, I. Mahadevan, A. Coutts, J. P. Bothma, B. J. Powell, R. H. McKenzie, S. C. Smith, and P. Meredith, "Convergent proton-transfer photocycles violate mirror-image symmetry in a key melanin monomer," Journal of the American Chemical Society, vol. 129, no. 21, pp. 6672-6673, 2007. 
[48] M. Gauden, A. Pezzella, L. Panzella, M. Neves-Petersen, E. Skovsen, S. B. Petersen, K. Mullen, A. Napolitano, M. d'Ischia, and V. Sundstrom, "Role of solvent, ph, and molecular size in excited-state deactivation of key eumelanin building blocks: implications for melanin pigment photostability," Journal of the American Chemical Society, vol. 130, no. 50, pp. 17038-17043, 2008.

[49] M. Gauden, A. Pezzella, L. Panzella, A. Napolitano, M. d'Ischia, and V. Sundstrom, "Ultrafast excited state dynamics of 5, 6-dihydroxyindole, a key eumelanin building block: nonradiative decay mechanism," The Journal of Physical Chemistry B, vol. 113, no. 37, pp. 12575-12580, 2009.

[50] A. Corani, A. Huijser, T. Gustavsson, D. Markovitsi, P.-Å. Malmqvist, A. Pezzella, M. d'Ischia, and V. Sundstrom, "Superior photoprotective motifs and mechanisms in eumelanins uncovered," Journal of the American Chemical Society, vol. 136, no. 33, pp. 11626-11635, 2014.

[51] J. J. Nogueira, A. Corani, A. El Nahhas, A. Pezzella, M. d'Ischia, L. Gonzalez, and V. Sundstrom, "Sequential proton-coupled electron transfer mediates excitedstate deactivation of a eumelanin building block," The journal of physical chemistry letters, vol. 8, no. 5, pp. 1004-1008, 2017.

[52] D. R. Weinberg, C. J. Gagliardi, J. F. Hull, C. F. Murphy, C. A. Kent, B. C. Westlake, A. Paul, D. H. Ess, D. G. McCafferty, and T. J. Meyer, "Proton-coupled electron transfer," Chemical Reviews, vol. 112, no. 7, pp. 4016-4093, 2012.

[53] G. D. Reid and K. Wynne, “Ultrafast laser technology and spectroscopy,” Encyclopedia of Analytical chemistry, 2000.

[54] P. Kukura, D. W. McCamant, S. Yoon, D. B. Wandschneider, and R. A. Mathies, "Structural observation of the primary isomerization in vision with femtosecond-stimulated raman," Science, vol. 310, no. 5750, pp. 1006-1009, 2005.

[55] P. Kukura, D. W. McCamant, and R. A. Mathies, "Femtosecond stimulated raman spectroscopy," Annu. Rev. Phys. Chem., vol. 58, pp. 461-488, 2007.

[56] D. W. McCamant, P. Kukura, and R. A. Mathies, "Femtosecond broadband stimulated raman: A new approach for high-performance vibrational spectroscopy," Applied spectroscopy, vol. 57, no. 11, pp. 1317-1323, 2003.

[57] D. W. McCamant, P. Kukura, S. Yoon, and R. A. Mathies, "Femtosecond broadband stimulated raman spectroscopy: Apparatus and methods," Review of scientific instruments, vol. 75, no. 11, pp. 4971-4980, 2004. 
[58] S.-Y. Lee, D. Zhang, D. W. McCamant, P. Kukura, and R. A. Mathies, “Theory of femtosecond stimulated raman spectroscopy," The Journal of chemical physics, vol. 121, no. 8, pp. 3632-3642, 2004.

[59] Victoris University of Wellington, "Victoria university of wellington." https://www.wgtn.ac.nz/scps/research/research-groups/ Ultra-fast-laser/facilities, 2020. [Online; accessed 11-April-2018].

[60] R. W. Boyd, Nonlinear optics. Academic press, 2003.

[61] e. P. Franken, A. E. Hill, C. e. Peters, and G. Weinreich, "Generation of optical harmonics," Physical Review Letters, vol. 7, no. 4, p. 118, 1961.

[62] I. Gražulevičiūtè, M. Skeivytè, E. Keblytė, J. Galinis, G. Tamošauskas, and A. Dubietis, "Supercontinuum generation in yag and sapphire with picosecond laser pulses," Lithuanian Journal of Physics, vol. 55, no. 2, 2015.

[63] Y. Shen, Fundamentals of sum-frequency spectroscopy. Cambridge University Press, 2016.

[64] H. Liu, J. Yao, and A. Puri, "Second and third harmonic generation in bbo by femtosecond ti: sapphire laser pulses," Optics communications, vol. 109, no. 1-2, pp. 139-144, 1994.

[65] A. Couairon and A. Mysyrowicz, "Femtosecond filamentation in transparent media," Physics Reports, vol. 441, no. 2-4, pp. 47-189, 2007.

[66] A. Dharmadhikari, F. Rajgara, and D. Mathur, "Systematic study of highly efficient white light generation in transparent materials using intense femtosecond laser pulses," Applied Physics B, vol. 80, no. 1, pp. 61-66, 2005.

[67] J. A. Dharmadhikari, R. A. Deshpande, A. Nath, K. Dota, D. Mathur, and A. K. Dharmadhikari, "Effect of group velocity dispersion on supercontinuum generation and filamentation in transparent solids," Applied Physics B, vol. 117, no. 1, pp. 471-479, 2014.

[68] P. J. Johnson, V. I. Prokhorenko, and R. D. Miller, "Stable uv to ir supercontinuum generation in calcium fluoride with conserved circular polarization states," Optics Express, vol. 17, no. 24, pp. 21488-21496, 2009.

[69] J. Yang and G. Mu, "Multi-dimensional observation of white-light filaments generated by femtosecond laser pulses in condensed medium," Optics express, vol. 15, no. 8, pp. 4943-4952, 2007. 
[70] J.-C. Diels and W. Rudolph, Ultrashort laser pulse phenomena. Elsevier, 2006.

[71] R. Trebino, K. W. DeLong, D. N. Fittinghoff, J. N. Sweetser, M. A. Krumbügel, B. A. Richman, and D. J. Kane, "Measuring ultrashort laser pulses in the timefrequency domain using frequency-resolved optical gating," Review of Scientific Instruments, vol. 68, no. 9, pp. 3277-3295, 1997.

[72] P. Sprangle, J. Penano, and B. Hafizi, "Propagation of intense short laser pulses in the atmosphere," Physical Review E, vol. 66, no. 4, p. 046418, 2002.

[73] S. Chin, A. Brodeur, S. Petit, O. Kosareva, and V. Kandidov, "Filamentation and supercontinuum generation during the propagation of powerful ultrashort laser pulses in optical media (white light laser)," Journal of Nonlinear Optical Physics $\mathcal{E}$ Materials, vol. 8, no. 01, pp. 121-146, 1999.

[74] D. Polli, D. Brida, S. Mukamel, G. Lanzani, and G. Cerullo, "Effective temporal resolution in pump-probe spectroscopy with strongly chirped pulses," Physical Review A, vol. 82, no. 5, p. 053809, 2010.

[75] C. V. Raman and K. S. Krishnan, "The optical analogue of the compton effect," Nature, vol. 121, no. 3053, p. 711, 1928.

[76] C. Raman and K. Krishnan, "The negative absorption of radiation," Nature, vol. 122, no. 3062, pp. 12-13, 1928.

[77] P. Graves and D. Gardiner, "Practical raman spectroscopy," Springer, 1989.

[78] M. Fujiwara, H. Hamaguchi, and M. Tasumi, "Measurements of spontaneous raman scattering with nd: Yag 1064-nm laser light," Applied spectroscopy, vol. 40, no. 2, pp. 137-139, 1986.

[79] G. Eckhardt, R. Hellwarth, F. McClung, S. Schwarz, D. Weiner, and E. Woodbury, "Stimulated raman scattering from organic liquids," Physical Review Letters, vol. 9, no. 11, p. 455, 1962.

[80] N. Bloembergen, "The stimulated raman effect," American Journal of Physics, vol. 35, no. 11, pp. 989-1023, 1967.

[81] D. Von der Linde, M. Maier, and W. Kaiser, "Quantitative investigations of the stimulated raman effect using subnanosecond light pulses," Physical review, vol. 178, no. 1, p. 11, 1969. 
[82] J. J. McGarvey, P. Callaghan, C. G. Coates, J. R. Schoonover, J. M. Kelly, L. Jacquet, and K. C. Gordon, "Comment on "resonance raman investigation of [ru (phen) 2 (dppz) 2+] and related complexes in water and in the presence of dna"," The Journal of Physical Chemistry B, vol. 102, no. 30, pp. 5941-5942, 1998.

[83] A. Owyoung and E. D. Jones, "Stimulated raman spectroscopy using low-power cw lasers," Optics letters, vol. 1, no. 5, pp. 152-154, 1977.

[84] D. R. Dietze and R. A. Mathies, "Femtosecond stimulated raman spectroscopy," ChemPhysChem, vol. 17, no. 9, pp. 1224-1251, 2016.

[85] D. P. Hoffman and R. A. Mathies, "Femtosecond Stimulated Raman Exposes the Role of Vibrational Coherence in Condensed-Phase Photoreactivity," Accounts of Chemical Research, vol. 49, no. 4, pp. 616-625, 2016.

[86] S. Shim, C. M. Stuart, and R. A. Mathies, "Resonance raman cross-sections and vibronic analysis of rhodamine $6 \mathrm{~g}$ from broadband stimulated raman spectroscopy," ChemPhysChem, vol. 9, no. 5, pp. 697-699, 2008.

[87] H. Kuramochi, S. Takeuchi, and T. Tahara, "Ultrafast structural evolution of photoactive yellow protein chromophore revealed by ultraviolet resonance femtosecond stimulated raman spectroscopy," The Journal of Physical Chemistry Letters, vol. 3, no. 15, pp. 2025-2029, 2012.

[88] W. R. Silva, E. L. Keller, and R. R. Frontiera, “Determination of resonance raman cross-sections for use in biological sers sensing with femtosecond stimulated raman spectroscopy," Analytical chemistry, vol. 86, no. 15, pp. 7782-7787, 2014.

[89] C. Chen, L.-d. Zhu, and C. Fang, "Femtosecond stimulated raman line shapes: Dependence on resonance conditions of pump and probe pulses," Chinese Journal of Chemical Physics, vol. 31, no. 4, p. 492, 2018.

[90] J. Lee, J. R. Challa, and D. W. McCamant, "Pump power dependence in resonance femtosecond stimulated raman spectroscopy," Journal of Raman Spectroscopy, vol. 44, no. 9, pp. 1263-1272, 2013.

[91] R. R. Frontiera, S. Shim, and R. A. Mathies, "Origin of negative and dispersive features in anti-stokes and resonance femtosecond stimulated raman spectroscopy," The Journal of chemical physics, vol. 129, no. 6, p. 064507, 2008. 
[92] K. Thorn, N. Monahan, S. Prasad, K. Chen, and J. Hodgkiss, "Efficient and tunable spectral compression using frequency-domain nonlinear optics," Optics express, vol. 26, no. 21, pp. 28140-28149, 2018.

[93] F. S. Parker, Applications of infrared, Raman, and resonance Raman spectroscopy in biochemistry. Springer Science \& Business Media, 1983.

[94] M. Yoshizawa, Y. Hattori, and T. Kobayashi, "Femtosecond time-resolved resonance raman gain spectroscopy in polydiacetylene," Physical Review B, vol. 49, no. 18 , p. 13259, 1994.

[95] L. Zhu, W. Liu, and C. Fang, "A versatile femtosecond stimulated raman spectroscopy setup with tunable pulses in the visible to near infrared," Applied Physics Letters, vol. 105, no. 4, p. 041106, 2014.

[96] B. G. Oscar, C. Chen, W. Liu, L. Zhu, and C. Fang, "Dynamic raman line shapes on an evolving excited-state landscape: Insights from tunable femtosecond stimulated raman spectroscopy," The Journal of Physical Chemistry A, vol. 121, no. 29, pp. 5428-5441, 2017.

[97] S. Nihonyanagi, J. A. Mondal, S. Yamaguchi, and T. Tahara, "Structure and dynamics of interfacial water studied by heterodyne-detected vibrational sumfrequency generation," Annual review of physical chemistry, vol. 64, pp. 579-603, 2013.

[98] J. Lavoie, J. M. Donohue, L. G. Wright, A. Fedrizzi, and K. J. Resch, "Spectral compression of single photons," Nature Photonics, vol. 7, no. 5, p. 363, 2013.

[99] L. J. Richter, T. P. Petralli-Mallow, and J. C. Stephenson, “Vibrationally resolved sum-frequency generation with broad-bandwidth infrared pulses," Optics letters, vol. 23, no. 20, pp. 1594-1596, 1998.

[100] H.-P. Chuang and C.-B. Huang, "Wavelength-tunable spectral compression in a dispersion-increasing fiber," Optics letters, vol. 36, no. 15, pp. 2848-2850, 2011.

[101] Y.-S. Lin and C.-B. Huang, "Large-scale and structure-tunable laser spectral compression in an optical dispersion-increasing fiber," Optics Express, vol. 25, no. 15, pp. 18024-18030, 2017.

[102] F. Raoult, A. Boscheron, D. Husson, C. Sauteret, A. Modena, V. Malka, F. Dorchies, and A. Migus, "Efficient generation of narrow-bandwidth picosec- 
ond pulses by frequency doubling of femtosecond chirped pulses," Optics letters, vol. 23, no. 14, pp. 1117-1119, 1998.

[103] S. Laimgruber, H. Schachenmayr, B. Schmidt, W. Zinth, and P. Gilch, "A femtosecond stimulated raman spectrograph for the near ultraviolet," Applied Physics B, vol. 85, no. 4, pp. 557-564, 2006.

[104] S. Kovalenko, A. Dobryakov, and N. Ernsting, "An efficient setup for femtosecond stimulated raman spectroscopy," Review of Scientific Instruments, vol. 82, no. 6, p. 063102, 2011.

[105] M. Nejbauer, T. M. Kardaś, Y. Stepanenko, and C. Radzewicz, "Spectral compression of femtosecond pulses using chirped volume bragg gratings," Optics letters, vol. 41, no. 11, pp. 2394-2397, 2016.

[106] H. Luo, L. Qian, P. Yuan, and H. Zhu, "Generation of tunable narrowband pulses initiating from a femtosecond optical parametric amplifier," Optics Express, vol. 14, no. 22, pp. 10631-10635, 2006.

[107] G. Xu, L. Qian, T. Wang, H. Zhu, C. Zhu, and D. Fan, "Spectral narrowing and temporal expanding of femtosecond pulses by use of quadratic nonlinear processes," IEEE Journal of selected topics in quantum electronics, vol. 10, no. 1, pp. 174180, 2004.

[108] R. W. Boyd, Nonlinear optics. Elsevier, 2003.

[109] M. Marangoni, D. Brida, M. Quintavalle, G. Cirmi, F. Pigozzo, C. Manzoni, F. Baronio, A. Capobianco, and G. Cerullo, "Narrow-bandwidth picosecond pulses by spectral compression of femtosecond pulses in a second-order nonlinear crystal," Optics Express, vol. 15, no. 14, pp. 8884-8891, 2007.

[110] C. Radzewicz, Y. Band, G. Pearson, and J. Krasinski, "Short pulse nonlinear frequency conversion without group-velocity-mismatch broadening," Optics communications, vol. 117, no. 3-4, pp. 295-302, 1995.

[111] A. Monmayrant, S. Weber, and B. Chatel, "A newcomer's guide to ultrashort pulse shaping and characterization," Journal of Physics B: Atomic, Molecular and Optical Physics, vol. 43, no. 10, p. 103001, 2010.

[112] M. Born and E. Wolf, Principles of optics: electromagnetic theory of propagation, interference and diffraction of light. Elsevier, 2013. 
[113] M. Born and E. Wolf, Principles of optics: electromagnetic theory of propagation, interference and diffraction of light. Elsevier, 2013.

[114] J. W. Shirley, "An early experimental determination of snell's law," American Journal of Physics, vol. 19, no. 9, pp. 507-508, 1951.

[115] K. Chen, J. K. Gallaher, A. J. Barker, and J. M. Hodgkiss, “Transient grating photoluminescence spectroscopy: an ultrafast method of gating broadband spectra," The journal of physical chemistry letters, vol. 5, no. 10, pp. 1732-1737, 2014.

[116] M. Marangoni, D. Brida, M. Quintavalle, G. Cirmi, F. Pigozzo, C. Manzoni, F. Baronio, A. Capobianco, and G. Cerullo, "Narrow-bandwidth picosecond pulses by spectral compression of femtosecond pulses in a second-order nonlinear crystal," Optics express, vol. 15, no. 14, pp. 8884-8891, 2007.

[117] P. A. Cornelius, "Spontaneous and stimulated raman studies of vibrational dephasing in condensed phases,"

[118] B. Sen and S. Mandal*, "Squeezed states in spontaneous raman and in stimulated raman processes," Journal of Modern Optics, vol. 52, no. 13, pp. 1789-1807, 2005.

[119] M. Plewicki and R. Levis, "Femtosecond stimulated raman spectroscopy of methanol and acetone in a noncollinear geometry using a supercontinuum probe," JOSA B, vol. 25, no. 10, pp. 1714-1719, 2008.

[120] H. Enqvist, "A setup for efficient frequency tripling of high-power femtosecond lasers pulses," Lund Reports in Atomic Physics, 2004.

[121] Y. An, Q. Sun, Y. Liu, C. Li, and Z.-Q. Wang, "The design of astigmatism-free crossed czerny-turner spectrometer," Optik, vol. 124, no. 16, pp. 2539-2543, 2013.

[122] Isben Photonics, "Spectrometer design guide." https://ibsen.com/ technology/spectrometer-design-guide/, 2018. [Online; accessed 20-April-2017].

[123] H. Noda, T. Namioka, and M. Seya, "Geometric theory of the grating," JOSA, vol. 64, no. 8, pp. 1031-1036, 1974.

[124] J. E. Harvey and C. L. Vernold, "Description of diffraction grating behavior in direction cosine space," Applied optics, vol. 37, no. 34, pp. 8158-8159, 1998. 
[125] K. C. Wilson, B. Lyons, R. Mehlenbacher, R. Sabatini, and D. W. McCamant, "Two-dimensional femtosecond stimulated raman spectroscopy: Observation of cascading raman signals in acetonitrile," The Journal of chemical physics, vol. 131, no. 21, p. 214502, 2009.

[126] P. H. Eilers, "Parametric time warping," Analytical chemistry, vol. 76, no. 2, pp. 404-411, 2004.

[127] J. J. de Rooi, O. Devos, M. Sliwa, C. Ruckebusch, and P. H. Eilers, "Mixture models for two-dimensional baseline correction, applied to artifact elimination in time-resolved spectroscopy," Analytica chimica acta, vol. 771, pp. 7-13, 2013.

[128] J. Lundberg, "Lifting the crown—citation z-score," Journal of informetrics, vol. 1, no. 2, pp. 145-154, 2007.

[129] S. Banerjee and A. Roy, Linear algebra and matrix analysis for statistics. Crc Press, 2014.

[130] E. J. Ientilucci, "Using the singular value decomposition," Rochester Institute of Technology, Rochester, New York, United States, Technical Report, 2003.

[131] H. Satzger and W. Zinth, "Visualization of transient absorption dynamicstowards a qualitative view of complex reaction kinetics," Chemical Physics, vol. 295, no. 3, pp. 287-295, 2003.

[132] M. Garrido, F. Rius, and M. Larrechi, "Multivariate curve resolution-alternating least squares (mcr-als) applied to spectroscopic data from monitoring chemical reactions processes," Analytical and bioanalytical chemistry, vol. 390, no. 8, pp. 2059-2066, 2008.

[133] R. G. Parr, "Density functional theory of atoms and molecules," in Horizons of quantum chemistry, pp. 5-15, Springer, 1980.

[134] J.-M. Combes, P. Duclos, and R. Seiler, "The born-oppenheimer approximation," in Rigorous atomic and molecular physics, pp. 185-213, Springer, 1981.

[135] M. Tsuboi, "Infrared and raman spectroscopy," Basic principles in nucleic acid chemistry, vol. 1, pp. 399-452, 1974.

[136] E. Kim, L. Panzella, A. Napolitano, and G. F. Payne, “Redox activities of melanins investigated by electrochemical reverse engineering: Implications for their roles 
in oxidative stress," Journal of Investigative Dermatology, vol. 140, no. 3, pp. 537$543,2020$.

[137] P. Ghosh and D. Ghosh, "Non-radiative decay of an eumelanin monomer: to be or not to be planar," Physical Chemistry Chemical Physics, vol. 21, no. 12, pp. 6635$6642,2019$.

[138] D. Bebelaar, "Time resolved molecular spectroscopy using high power solid state lasers in pulse transmission mode. a re-examination of the sn $\leftarrow$ s1 spectra of naphthalene and anthracene," Chemical Physics, vol. 3, no. 2, pp. 205-216, 1974.

[139] V. Svetlichnyi, T. Kopylova, G. Mayer, and I. Lapin, "Transient absorption of organic molecules under high-power laser excitation," Russian physics journal, vol. 48, no. 9, pp. 901-906, 2005.

[140] P. Bonancía, M. C. Jiménez, and M. A. Miranda, "Transient absorption spectroscopy detection of sensitized delayed fluorescence in chiral benzophenone/naphthalene systems," Chemical Physics Letters, vol. 515, no. 1-3, pp. 194-196, 2011.

[141] X. Wang, W. G. Kofron, S. Kong, C. Rajesh, D. A. Modarelli, and E. C. Lim, “Transient absorption probe of intermolecular triplet excimer of naphthalene in fluid solutions: Identification of the species based on comparison to the intramolecular triplet excimers of covalently-linked dimers," The Journal of Physical Chemistry A, vol. 104, no. 7, pp. 1461-1465, 2000.

[142] H. Shinohara, Y. Yamakita, and K. Ohno, "Raman spectra of polycyclic aromatic hydrocarbons. comparison of calculated raman intensity distributions with observed spectra for naphthalene, anthracene, pyrene, and perylene," Journal of Molecular Structure, vol. 442, no. 1-3, pp. 221-234, 1998.

[143] A. L. Sobolewski and W. Domcke, "Photophysics of eumelanin: Ab initio studies on the electronic spectroscopy and photochemistry of 5, 6-dihydroxyindole," ChemPhysChem, vol. 8, no. 5, pp. 756-762, 2007.

[144] M. Gauden, A. Pezzella, L. Panzella, A. Napolitano, M. d'Ischia, and V. Sundstrom, "Ultrafast excited state dynamics of 5, 6-dihydroxyindole, a key eumelanin building block: nonradiative decay mechanism," The Journal of Physical Chemistry B, vol. 113, no. 37, pp. 12575-12580, 2009. 
[145] K. C. Gross and P. G. Seybold, "Substituent effects on the physical properties and pka of phenol," International Journal of Quantum Chemistry, vol. 85, no. 4-5, pp. 569-579, 2001.

[146] B. G. Oscar, "Tracking excited state proton transfer and inhibition with ultrafast raman and transient absorption spectroscopy," 2017.

[147] P. Frank and P. General, “Schlenk line design and safety," 2011.

[148] D. N. Peles, E. Lin, K. Wakamatsu, S. Ito, and J. D. Simon, “Ultraviolet absorption coefficients of melanosomes containing eumelanin as related to the relative content of dhi and dhica," The Journal of Physical Chemistry Letters, vol. 1, no. 15, pp. 2391-2395, 2010.

[149] I. Galván, C. Araujo-Andrade, M. Marro, P. Loza-Alvarez, and K. Wakamatsu, "Raman spectroscopy quantification of eumelanin subunits in natural unaltered pigments," Pigment Cell \& Melanoma Research, vol. 31, no. 6, pp. 673-682, 2018.

[150] V. Capozzi, G. Perna, A. Gallone, P. Biagi, P. Carmone, A. Fratello, G. Guida, P. Zanna, and R. Cicero, "Raman and optical spectroscopy of eumelanin films," Journal of molecular structure, vol. 744, pp. 717-721, 2005.

[151] C. Artur, E. C. Le Ru, and P. G. Etchegoin, "Temperature dependence of the homogeneous broadening of resonant raman peaks measured by single-molecule surface-enhanced raman spectroscopy," The Journal of Physical Chemistry Letters, vol. 2, no. 23, pp. 3002-3005, 2011.

[152] D. Ricard, "Raman spectroscopy and inhomogeneous broadening," The Journal of chemical physics, vol. 116, no. 2, pp. 860-861, 2002.

[153] O. A. Lozhkina, V. I. Yudin, A. A. Murashkina, V. V. Shilovskikh, V. G. Davydov, R. Kevorkyants, A. V. Emeline, Y. V. Kapitonov, and D. W. Bahnemann, "Low inhomogeneous broadening of excitonic resonance in mapbbr3 single crystals," The journal of physical chemistry letters, vol. 9, no. 2, pp. 302-305, 2018.

[154] B. Armstrong, "Spectrum line profiles: the voigt function," Journal of Quantitative Spectroscopy and Radiative Transfer, vol. 7, no. 1, pp. 61-88, 1967.

[155] V. Capozzi, G. Perna, A. Gallone, P. Biagi, P. Carmone, A. Fratello, G. Guida, P. Zanna, and R. Cicero, "Raman and optical spectroscopy of eumelanin films," Journal of molecular structure, vol. 744, pp. 717-721, 2005. 
[156] G. Perna, M. Lasalvia, and V. Capozzi, "Vibrational spectroscopy of synthetic and natural eumelanin," Polymer International, vol. 65, no. 11, pp. 1323-1330, 2016.

[157] A. C. Ferrari and J. Robertson, "Interpretation of raman spectra of disordered and amorphous carbon," Physical review B, vol. 61, no. 20, p. 14095, 2000.

[158] S. Pisana, M. Lazzeri, C. Casiraghi, K. S. Novoselov, A. K. Geim, A. C. Ferrari, and F. Mauri, "Breakdown of the adiabatic born-oppenheimer approximation in graphene," Nature materials, vol. 6, no. 3, pp. 198-201, 2007.

[159] L. Soukup, I. Gregora, L. Jastrabik, and A. Koňáková, “Raman spectra and electrical conductivity of glassy carbon," Materials Science and Engineering: B, vol.11, no. 1-4, pp. 355-357, 1992.

[160] M. Lazzeri and F. Mauri, "Nonadiabatic kohn anomaly in a doped graphene monolayer," Physical review letters, vol. 97, no. 26, p. 266407, 2006.

[161] S. L. Howell, K. C. Gordon, M. R. Waterland, K. H. Leung, and D. L. Phillips, "Resonance raman excitation profile of a ruthenium (ii) complex of dipyrido [2, 3-a: 3 ', 2 '-c] phenazine," The Journal of Physical Chemistry A, vol. 110, no. 38, pp. 11194-11199, 2006.

[162] P. J. Walsh, K. C. Gordon, P. Wagner, and D. L. Officer, “Resonance raman studies of $\beta$-substituted porphyrin systems with unusual electronic absorption properties," ChemPhysChem, vol. 7, no. 11, pp. 2358-2365, 2006.

[163] P. K. Gordon, “University of otago." https://www.otago.ac.nz/ chemistry/index.html, 2020.

[164] S. Shionoya, "Photoluminescence," in Luminescence of solids, pp. 95-133, Springer, 1998.

[165] C. Pardanaud, C. Martin, P. Roubin, G. Giacometti, C. Hopf, T. Schwarz-Selinger, and W. Jacob, "Raman spectroscopy investigation of the $h$ content of heated hard amorphous carbon layers," Diamond and related materials, vol. 34, pp. 100-104, 2013.

[166] A. J. Bullen, K. E. O'Hara, D. G. Cahill, O. Monteiro, and A. Von Keudell, “Thermal conductivity of amorphous carbon thin films," Journal of Applied Physics, vol. 88 , no. 11, pp. 6317-6320, 2000. 
[167] The Engineering ToolBox, "The engineering toolbox." https://www. engineeringtoolbox.com/specific-heat-solids-d_154.html,

2020. [Online; accessed 10-March-2020].

[168] H. Kuramochi, S. Takeuchi, and T. Tahara, "Femtosecond time-resolved impulsive stimulated raman spectroscopy using sub-7-fs pulses: Apparatus and applications," Review of Scientific Instruments, vol. 87, no. 4, p. 043107, 2016.

[169] A. Aggarwal, "Riken laboratories 2-1 hirosawa, wako city, saitama prefecture 351-0198." http://www2.riken.jp/lab/spectroscopy/tahara-e. html, 2020.

[170] M. Citroni, "Real-time bond formation," Nature Reviews Chemistry, vol. 4, no. 2, pp. 64-64, 2020.

[171] T. Fujisawa, H. Kuramochi, H. Hosoi, S. Takeuchi, and T. Tahara, "Role of coherent low-frequency motion in excited-state proton transfer of green fluorescent protein studied by time-resolved impulsive stimulated raman spectroscopy," Journal of the American Chemical Society, vol. 138, no. 12, pp. 3942-3945, 2016.

[172] J. Kim, T. H. Yoon, and M. Cho, "Time-resolved impulsive stimulated raman spectroscopy with synchronized triple mode-locked lasers," The Journal of Physical Chemistry Letters, vol. 11, no. 8, pp. 2864-2869, 2020.

[173] S. Fujiyoshi, S. Takeuchi, and T. Tahara, "Time-resolved impulsive stimulated raman scattering from excited-state polyatomic molecules in solution," The Journal of Physical Chemistry A, vol. 107, no. 4, pp. 494-500, 2003.

[174] H. Kuramochi, S. Takeuchi, and T. Tahara, "Ultrafast photodissociation dynamics of diphenylcyclopropenone studied by time-resolved impulsive stimulated raman spectroscopy," Chemical Physics, vol. 512, pp. 88-92, 2018.

[175] H. Kuramochi, S. Takeuchi, H. Kamikubo, M. Kataoka, and T. Tahara, "Fifthorder time-domain raman spectroscopy of photoactive yellow protein for visualizing vibrational coupling in its excited state," Science advances, vol. 5, no. 6, p. eaau4490, 2019.

[176] S. Fujiyoshi, S. Takeuchi, and T. Tahara, "Time-resolved impulsive stimulated raman studies of 1, 1 '-binaphthyl in the excited state: Low-frequency vibrations and conformational relaxation," The Journal of Physical Chemistry A, vol. 108, no. 28, pp. 5938-5943, 2004. 
[177] H. Kuramochi, S. Takeuchi, K. Yonezawa, H. Kamikubo, M. Kataoka, and T. Tahara, "Probing the early stages of photoreception in photoactive yellow protein with ultrafast time-domain raman spectroscopy," Nature Chemistry, vol. 9, no. 7, p. 660, 2017.

[178] T. Fujisawa, H. Kuramochi, S. Takeuchi, and T. Tahara, "Time-resolved impulsive raman study of excited state structures of green fluorescent protein," in International Conference on Ultrafast Phenomena, pp. 08-Tue, Optical Society of America, 2014. 